BIBLIOTHECA

SCRTPTORVM CRABCORVM BR ROMAMORVM T $\triangle B$ N $B$ IANA

\title{
DIDYMUS
}

\section{IN DEMOSTHENEM COMMENTA}

\author{
EDIDERVNT
}

L. PEARSON ET S. STEPHENS

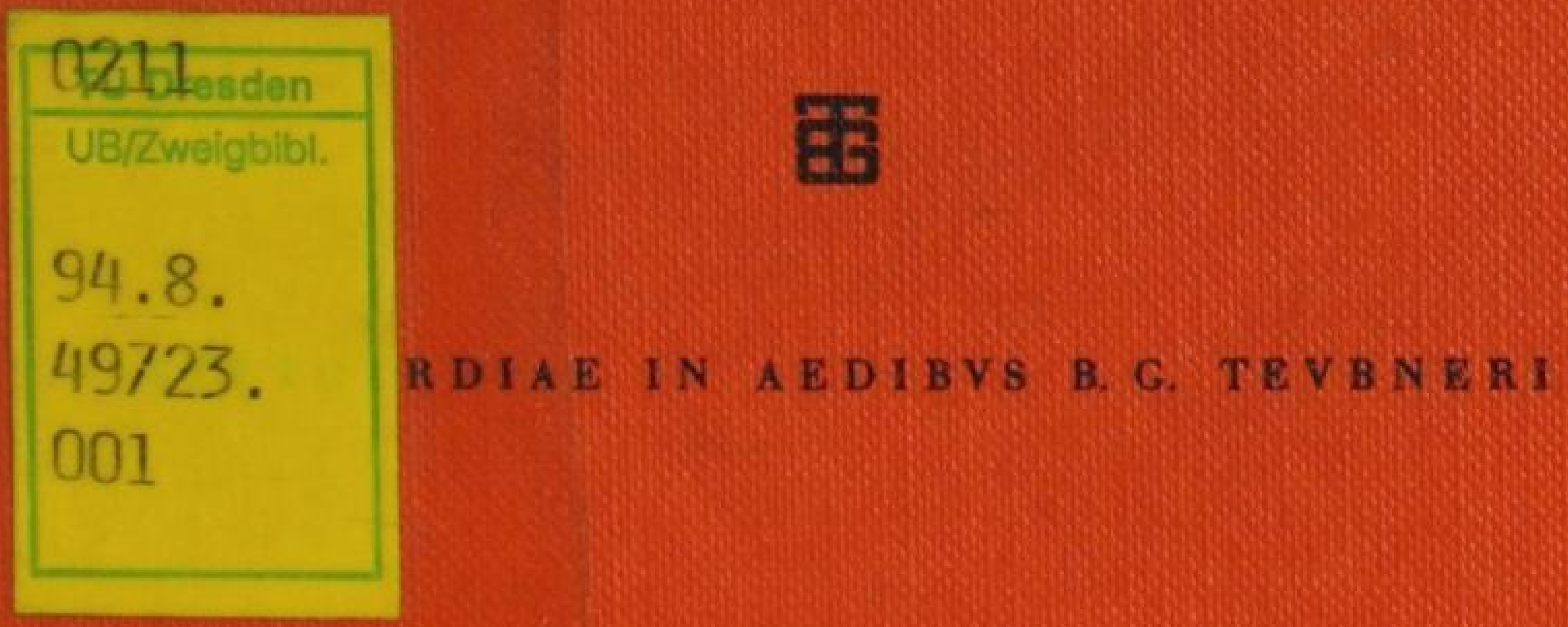




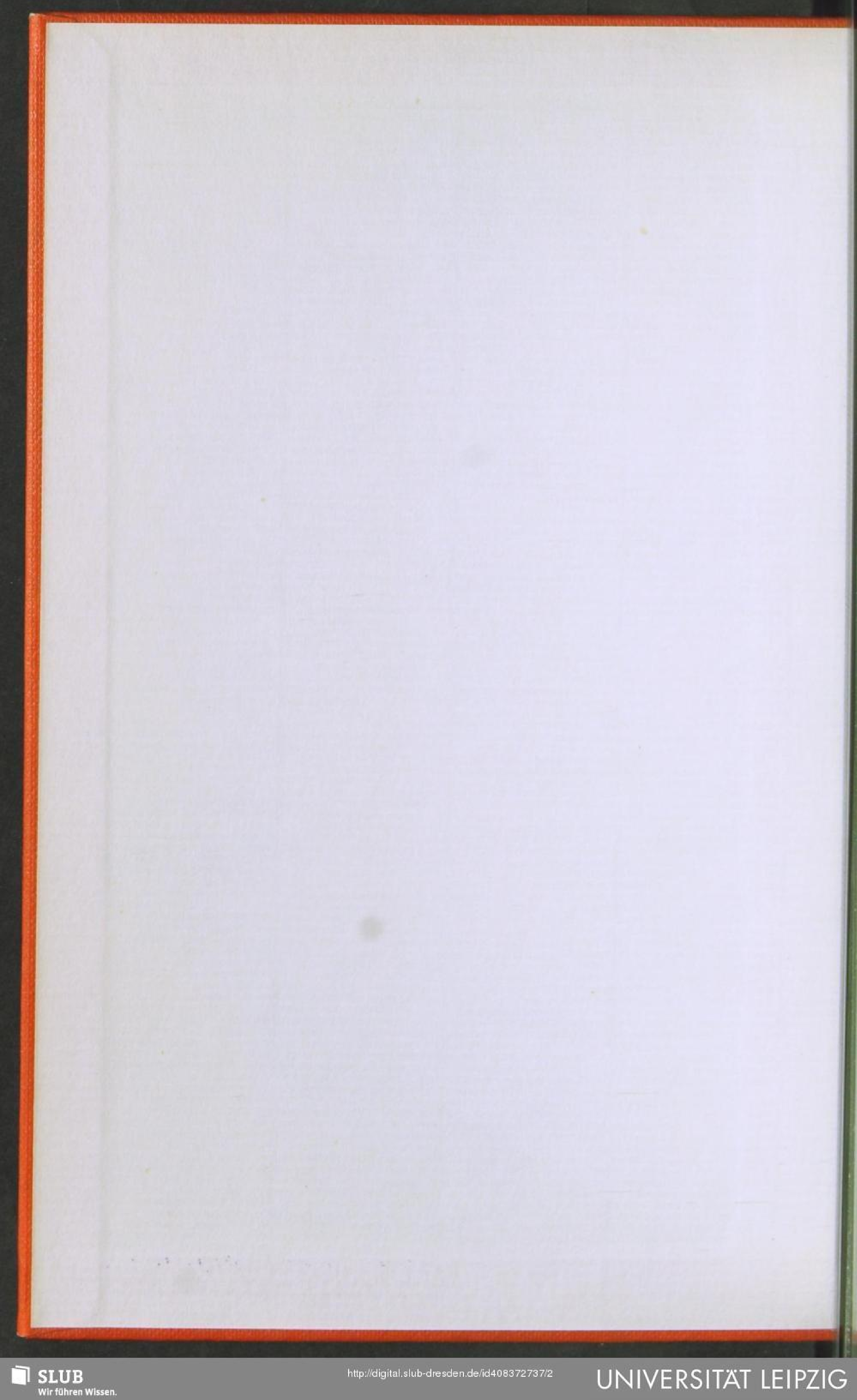




\title{
DIDYMI
}

\section{IN DEMOSTHENEM COMMENTA}

\author{
EDIDERVNT
}

LIONEL PEARSON ET SVSAN STEPHENS

\section{宙}

STVTGARDIAE IN AEDIBVS B. G.TEVBNERI MCMLXXXIII 


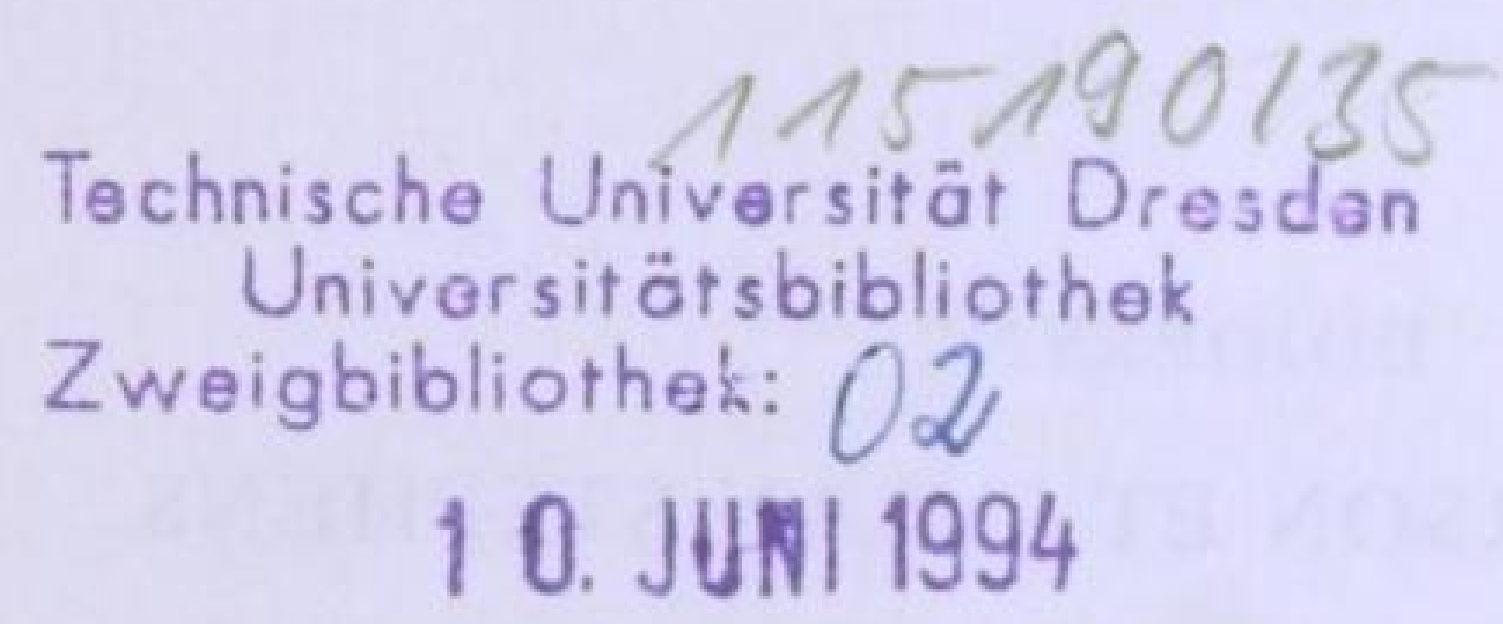

Gedruckt mit Unterstützung der Förderungs- und Beihilfefonds Wissenschaft der VG WORT GmbH, Goethestraße 49,8000 München 2

CIP-Kurztitelaufnahme der Deutschen Bibliothek

Didymus (Chalcenterus):

[In Demosthenem commenta]

Didymi in Demosthenem commenta / ed. Lionel

Pearson et Susan Stephens. - Stuttgardiae :

Teubner, 1983.

(Bibliotheca scriptorum Graecorum et

Romanorum Teubneriana)

ISBN 3-519-01269-3

NE: Pearson, Lionel [Hrsg.]

Das Werk ist urheberrechtlich geschützt. Die dadurch begründeten Rechte, besonders des Nachdrucks, der Wiedergabe auf photomechanischem oder ähnlichem Wege, der Speicherung und Auswertung in Datenverarbeitungsanlagen, bleiben, auch bei Verwertung von Teilen des Werkes, dem Verlag vorbehalten. Bei gewerblichen Zwecken dienender Vervielfältigung ist an den Verlag gemäß $\S 54$ UrhG eine Vergütung zu zahlen, deren Höhe mit dem Verlag zu vereinbaren ist.

(c) B.G. Teubner, Stuttgart 1983

Printed in Germany

Satz: Krebs-Gehlen Druckerei GmbH \& Co. KG, Hemsbach/Bergstr.

Druck und Bindung: Passavia Druckerei GmbH Passau
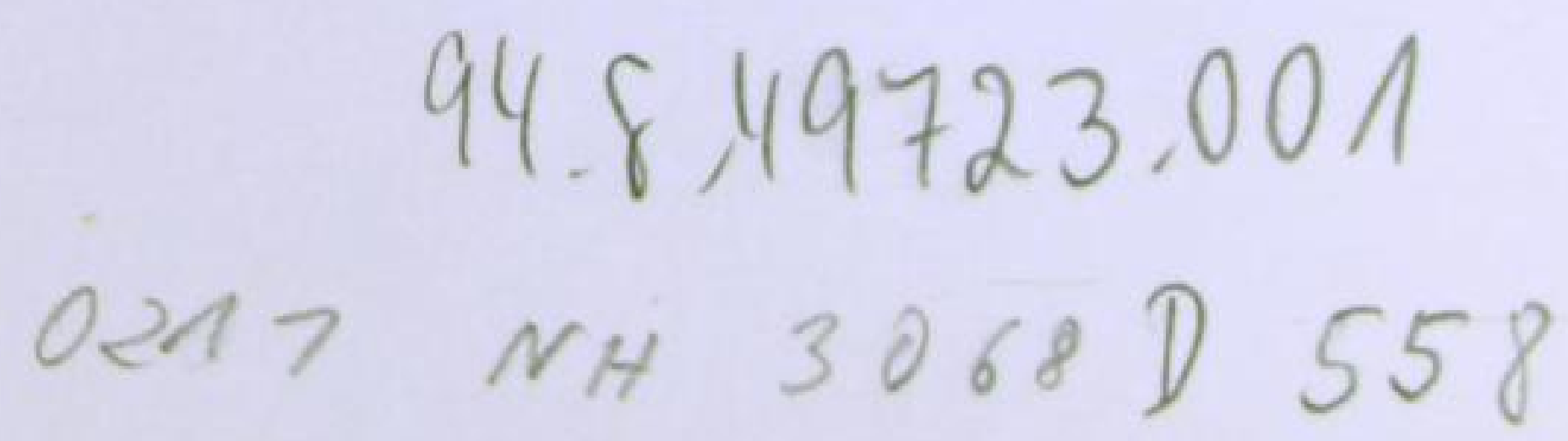


\section{$\Delta \mathrm{I} \Delta \mathrm{YMOY}$ \\ ФІ $\Lambda$ ІППІК $\Omega \mathrm{N} \bar{\Theta}-\overline{\mathrm{IB}}$}

Col. I

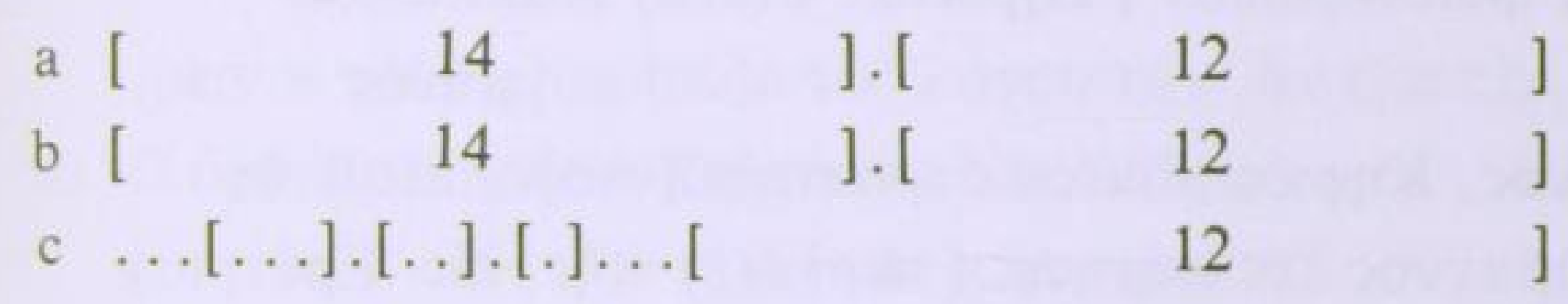

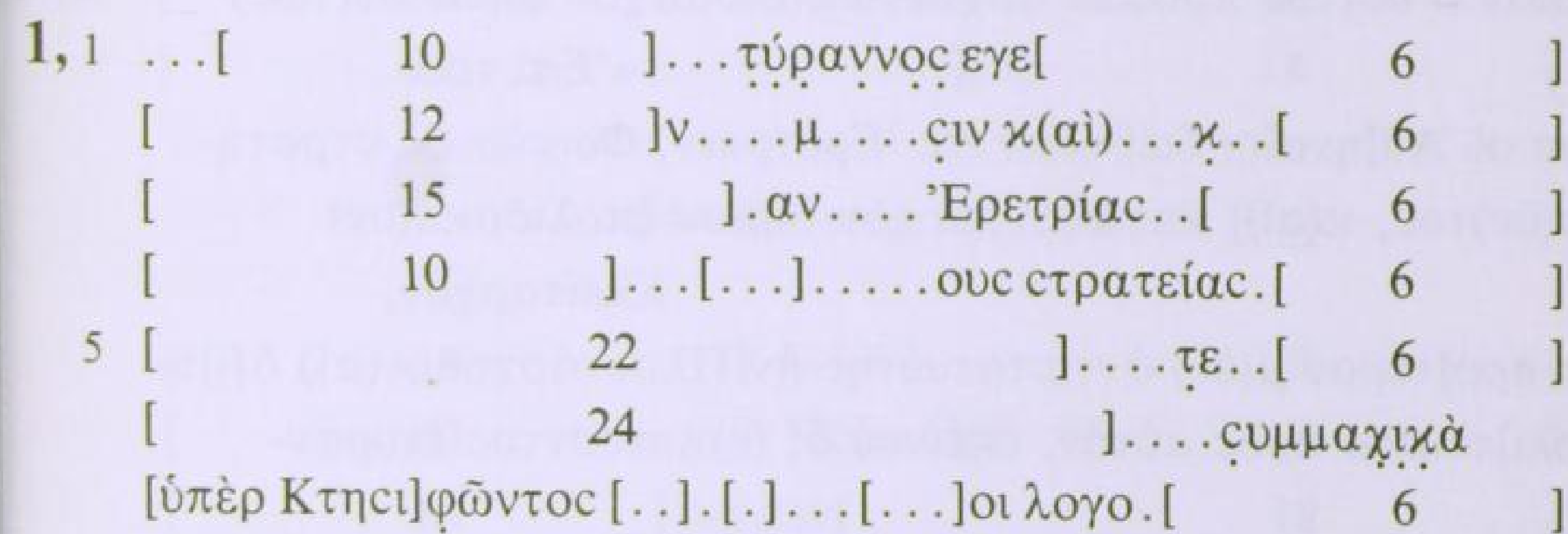

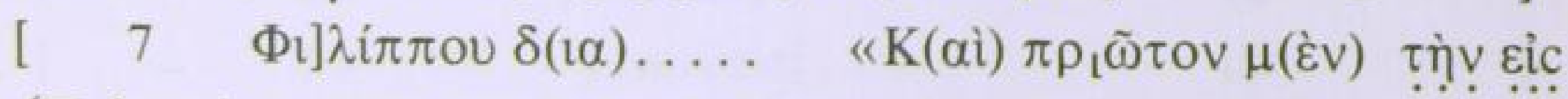

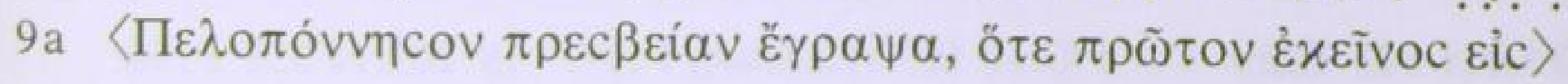




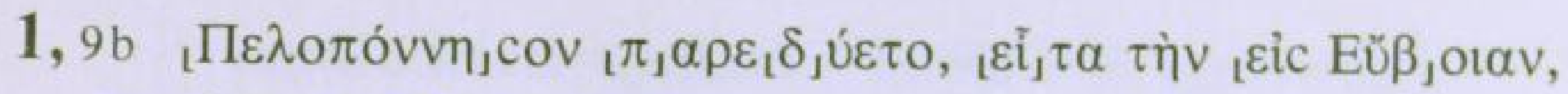

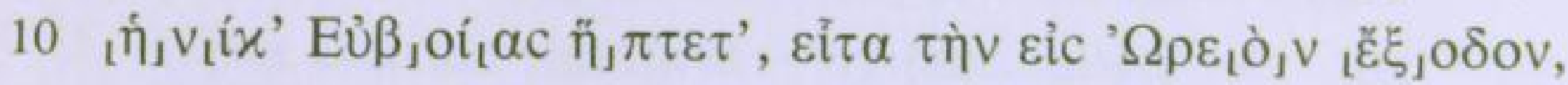


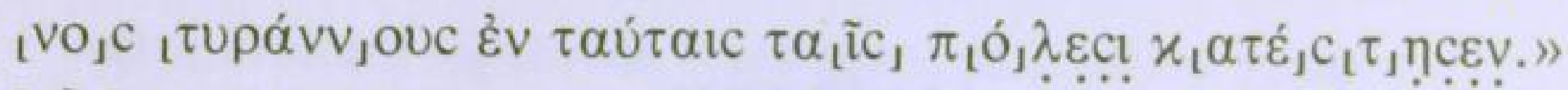

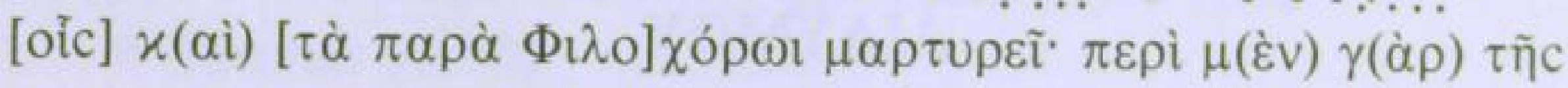

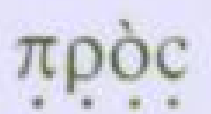

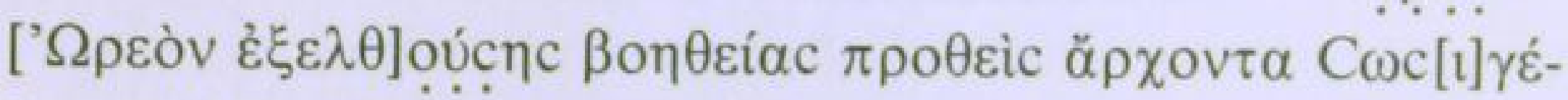

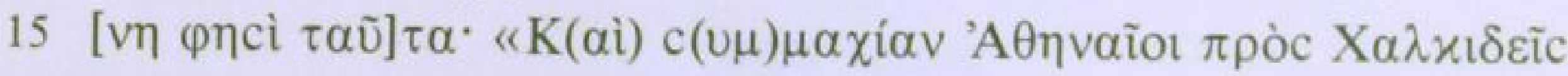
$\dot{\varepsilon} \pi \mathrm{ol}-$

[

$\mu$ пेòc

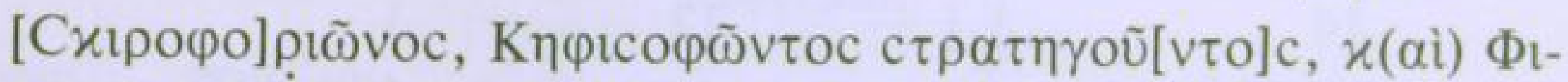

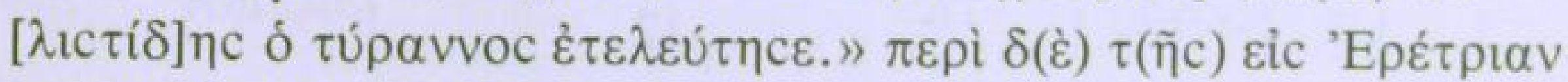

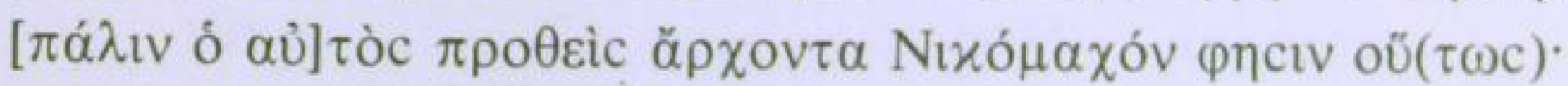

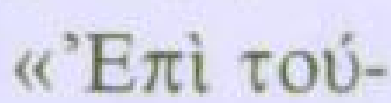

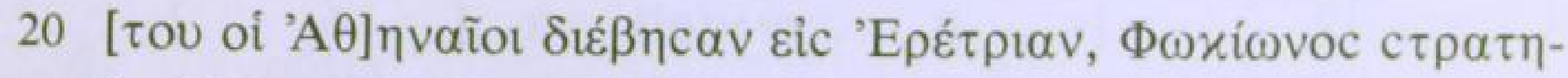

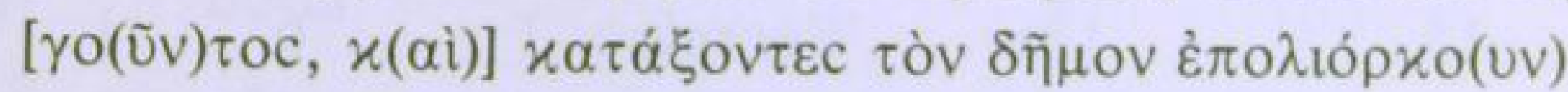

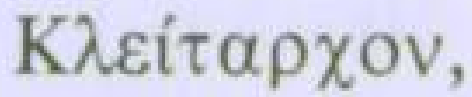

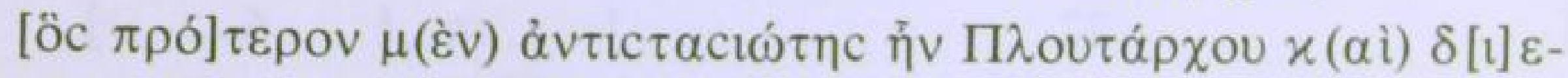

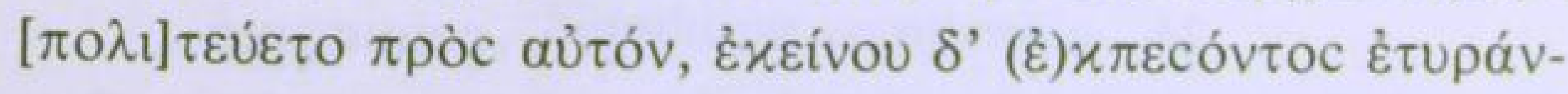




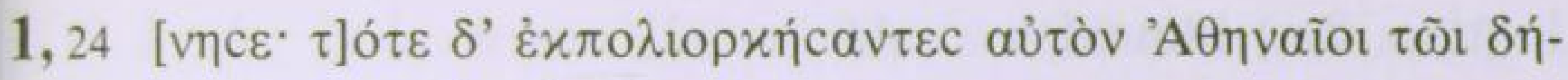

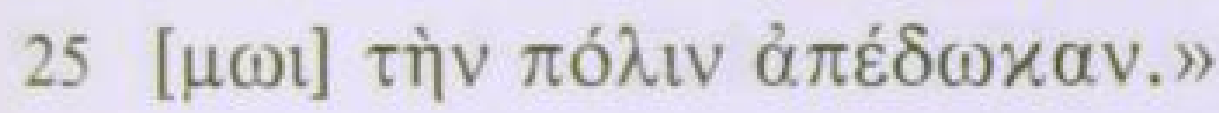

\section{$\overline{\mathrm{I}}$}

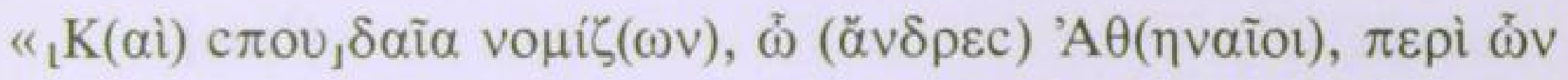
$\beta_{1} 0_{\jmath} \cup \lambda_{1} \varepsilon, \cup ́ \varepsilon c \theta \varepsilon, \chi(\alpha i) \alpha \alpha v \alpha \gamma-$

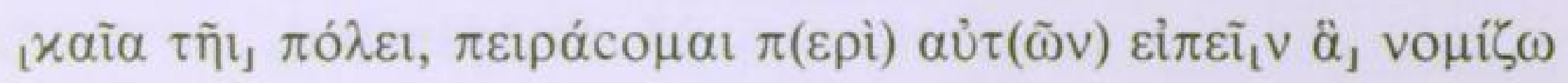
$\mathrm{c}(\nu \mu) \varphi \varepsilon$ -

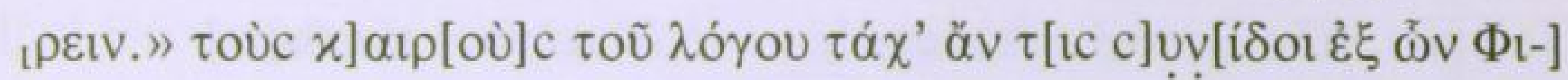

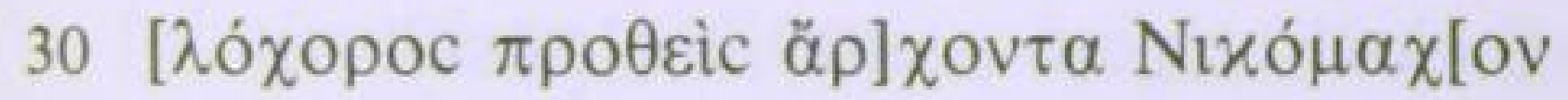

$\begin{array}{ll}\text { [ } & 15 \\ \text { [ } & 20 \\ \text { [ } & 24\end{array}$

$$
\begin{aligned}
& \text { ] } \eta[4] . \eta v \pi \rho \circ[ \\
& \text { ]. . [ }
\end{aligned}
$$

\begin{tabular}{|c|c|c|c|}
\hline [ & 18 & ]$\cdot \eta \cdot[$ & 18 \\
\hline [ & 20 & ].c[...] $\varepsilon \underline{[}$ & 14 \\
\hline [ & 16 & ]. $v \varepsilon v \omega v[$ & 18 \\
\hline 40 ! & 16 & ]. $v \omega v \varepsilon ı \tau[$ & 16 \\
\hline 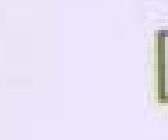 & 14 & ]... $\eta c \theta \varepsilon .[$ & 18 \\
\hline & 16 & ]$\pi$ oınc $\alpha .[$ & 16 \\
\hline 1 & 12 & ] . . . $\varepsilon \rho \omega \mu \varepsilon v[$ & 14 \\
\hline & 14 & ].cxouc $\alpha[$ & 14 \\
\hline
\end{tabular}

$\begin{array}{ll}12 & \text { ] } \\ 14 & \text { ] } \\ 12 & \text { ] }\end{array}$

\section{$34-36$ integri perierunt}




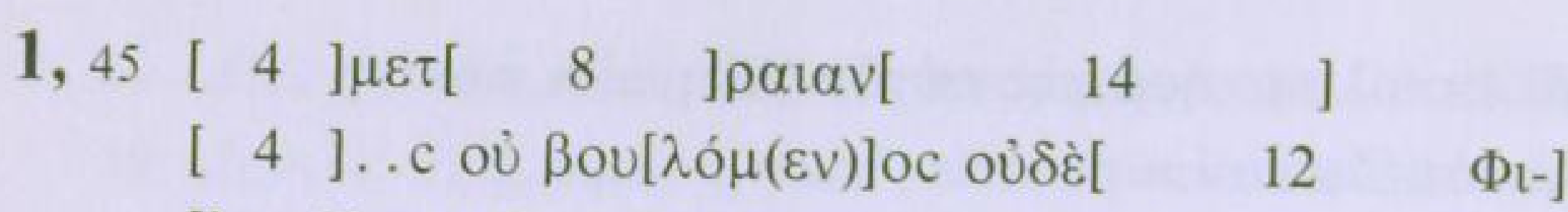

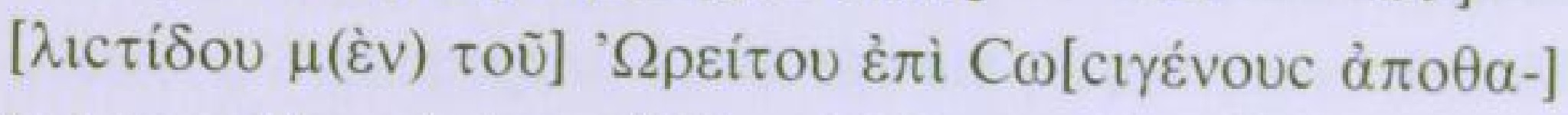

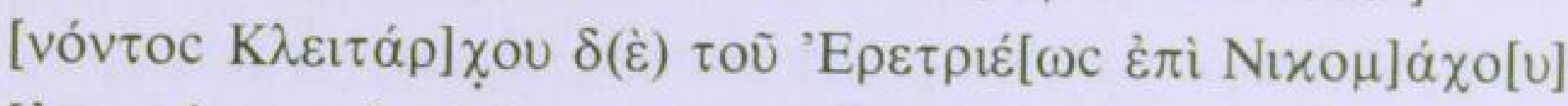

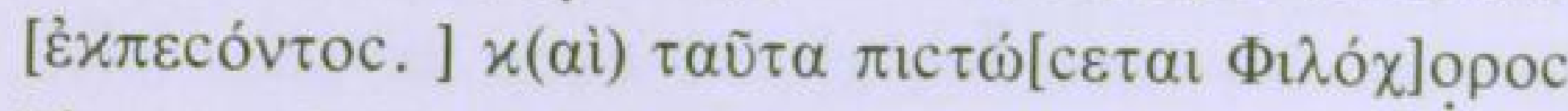

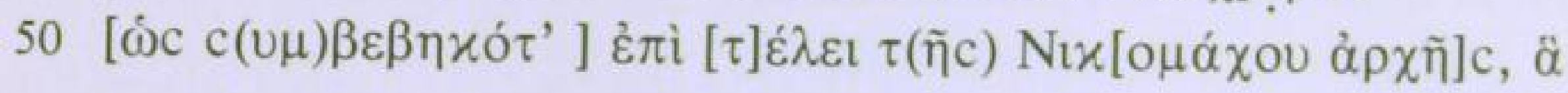

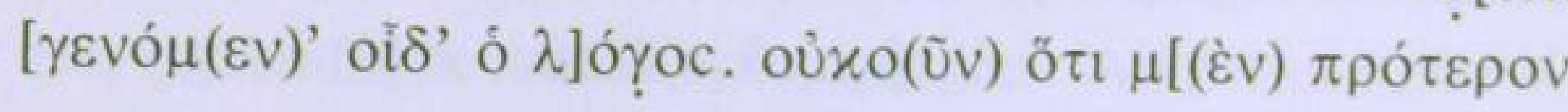
o[vั $\pi \omega]$ $\gamma \varepsilon \dot{\gamma} \gamma] \rho \alpha \tau\left[\alpha_{l}\right]$

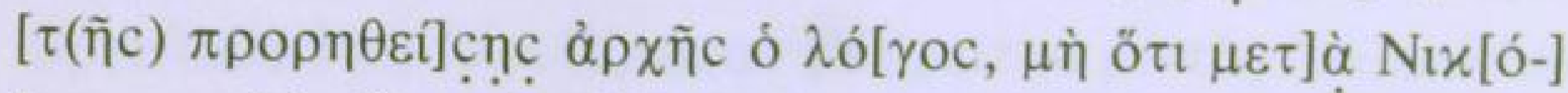

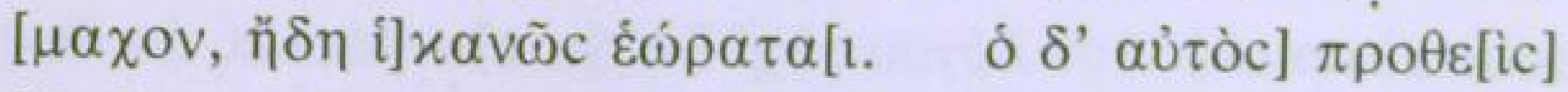

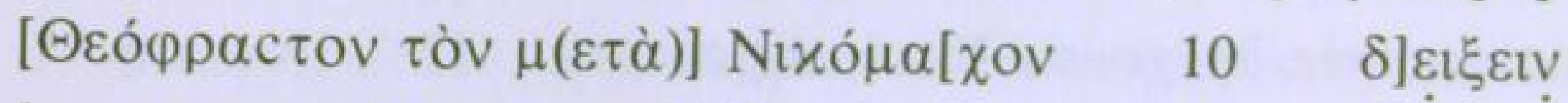

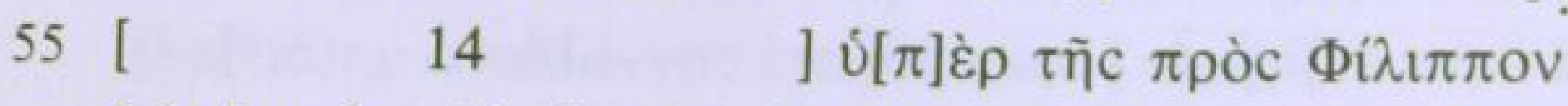

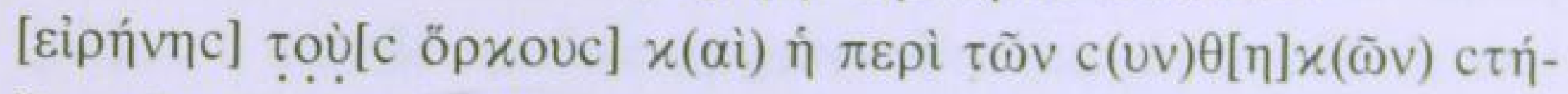

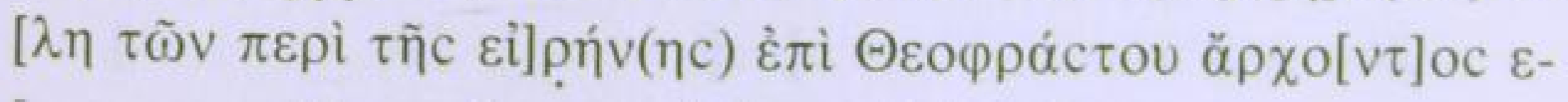

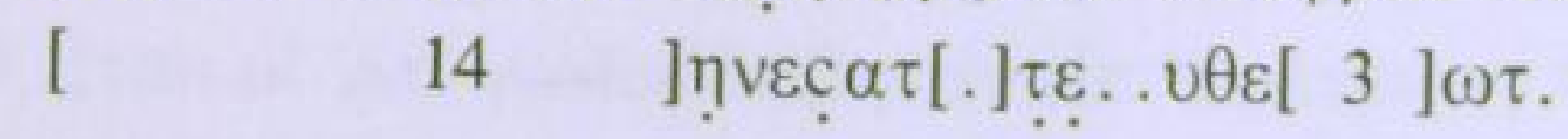




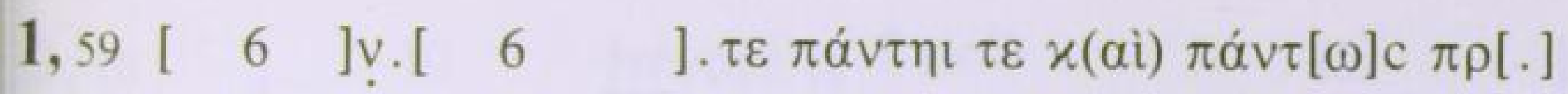

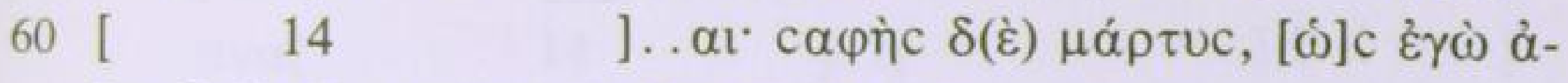

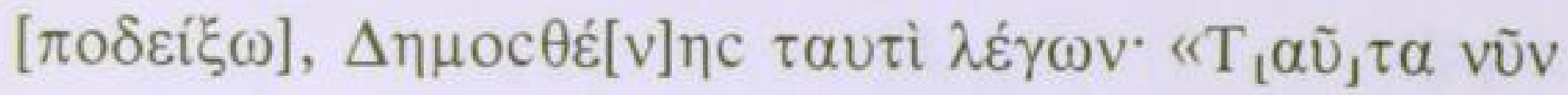

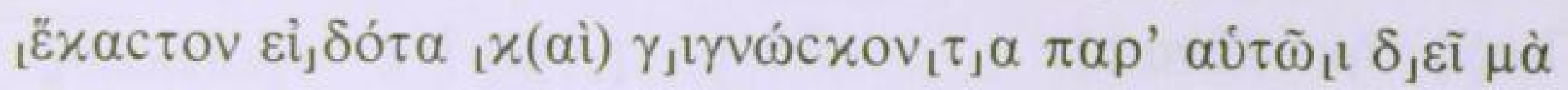

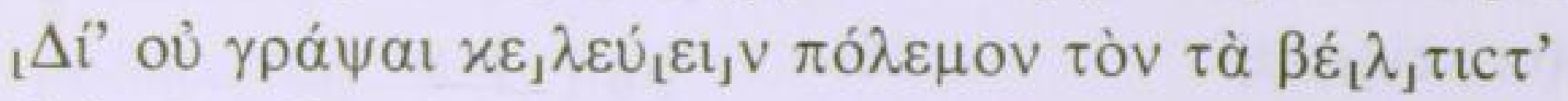

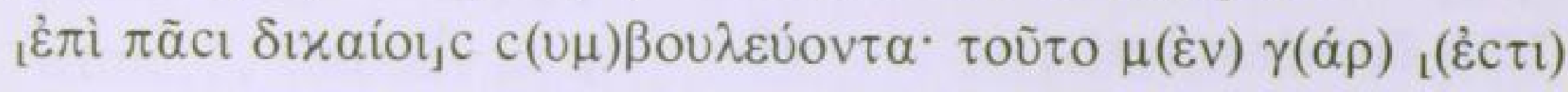

\section{$\lambda, \alpha \beta \varepsilon \bar{\varepsilon} v$}

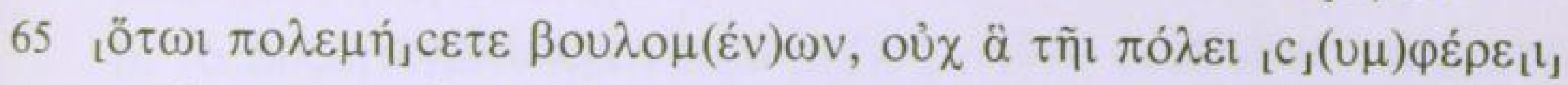

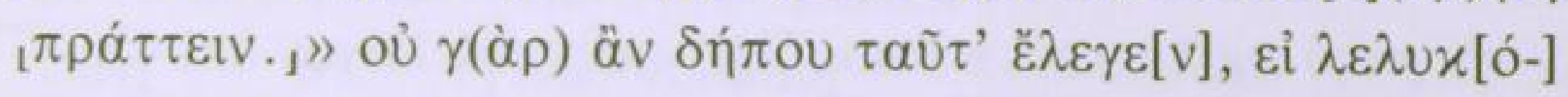

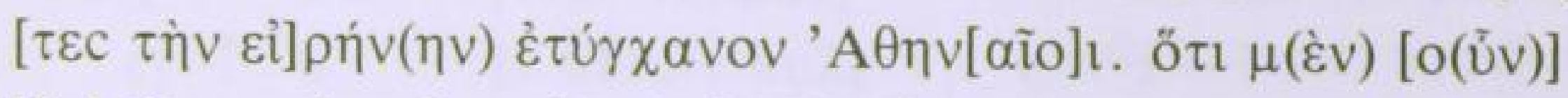

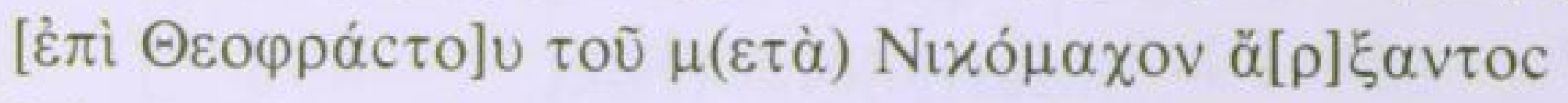

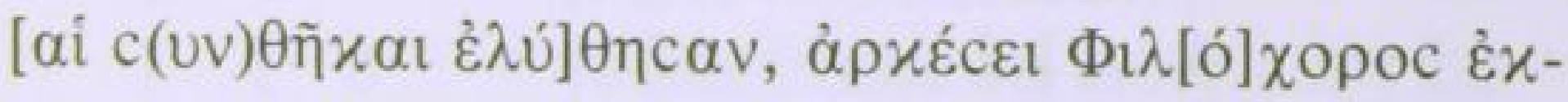

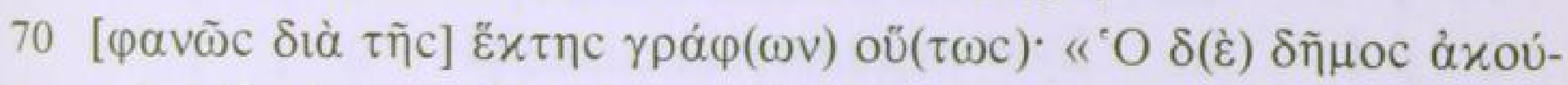

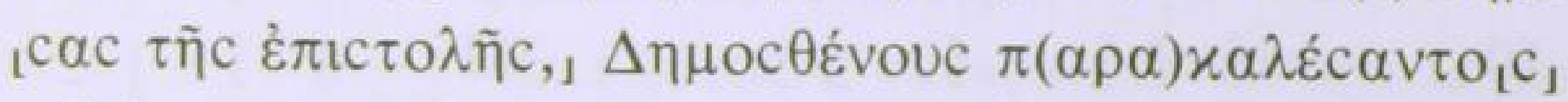

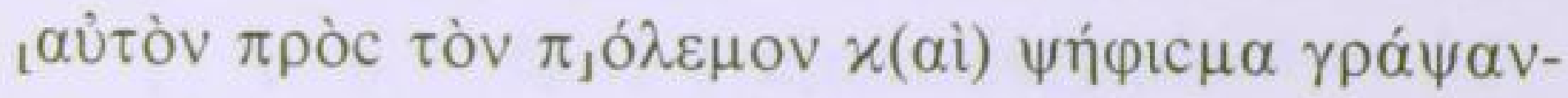

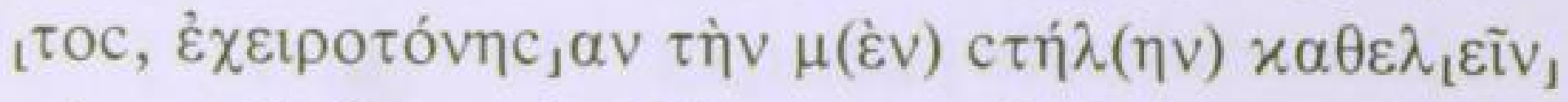

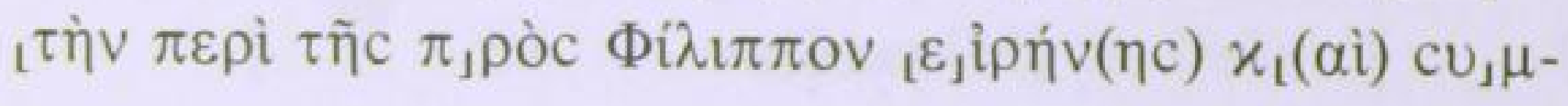


Col. 2

\begin{tabular}{|c|c|c|}
\hline a & Tívec oi $\pi \varepsilon \rho 1[$ & 18 \\
\hline b & 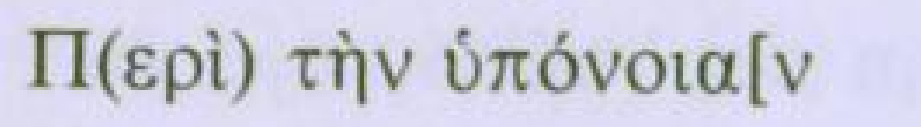 & 18 \\
\hline $\mathrm{c}$ & 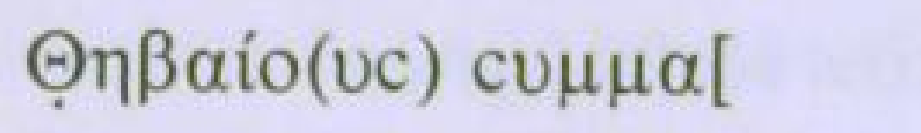 & 18 \\
\hline & 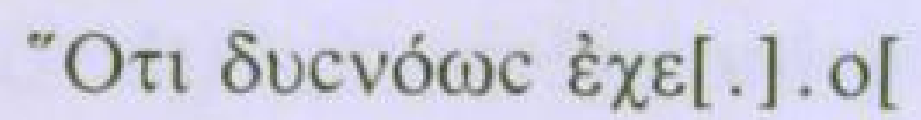 & 18 \\
\hline
\end{tabular}

2, $1 \mu \alpha \chi_{1} \hat{i}_{1} \alpha c \mathrm{c} \tau \alpha \theta \varepsilon \tilde{c} c \alpha v, v \alpha \tilde{v}_{1} c_{1} \delta(\grave{\varepsilon}) \pi \lambda \eta \rho o \tilde{v} \chi(\alpha i) \dot{\varepsilon} v \varepsilon \rho-$

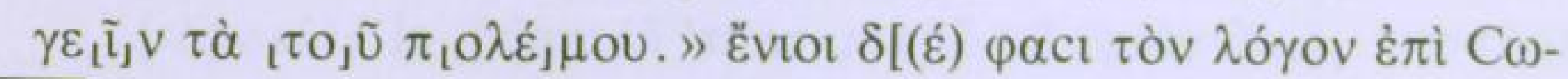

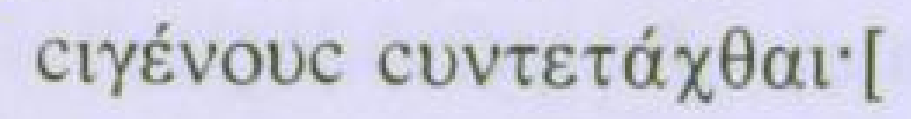
$\operatorname{\tau ov}[7$ ]ou [.] $7 \varepsilon 1 \delta \ldots \tau[$ 15 $\mu \alpha \lambda\left[\begin{array}{llll}7 & 7\end{array}\right] \cup ̣[\quad 4 \quad] \mu[$ 15

5 [ 3 ].[ 5 ] ] $5 \varepsilon$ ic $x(\alpha i) \eta \mu .$. [ 15

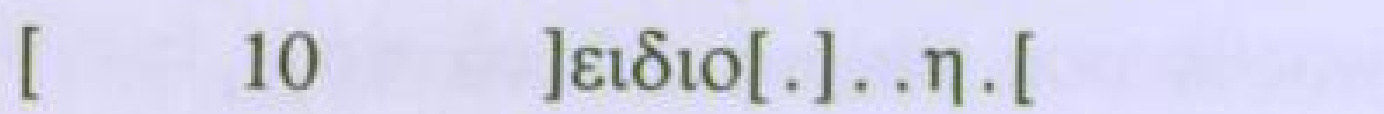

\begin{tabular}{|c|c|c|c|c|c|}
\hline & D & 10 & 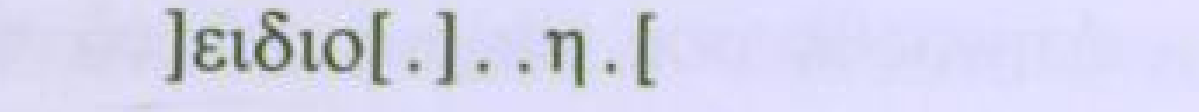 & 12 & \\
\hline & [ & 10 & ]. $\tau \alpha \pi \varepsilon \rho i ̀ ~ \alpha u \tau o[$ & 15 & \\
\hline & [ & 10 & ]$v$ ŏ $\tau \iota \pi \circ \lambda \operatorname{lo\rho }[x$ & 12 & \\
\hline \multirow[t]{5}{*}{10} & {[} & 10 & ]. $x(\alpha i)$ eic [M] $\alpha \underset{x}{\varepsilon \delta}[o v$ & 10 & \\
\hline & {[} & 12 & ]$\ldots \kappa \ldots \varkappa \eta$ & 15 & \\
\hline & {[} & 10 & ]..[.].c $\tau \rho \alpha \tau o u[$ & 12 & \\
\hline & {[} & 10 & 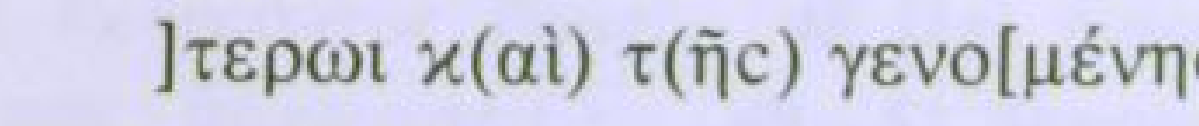 & & 10 \\
\hline & {[} & 7 & 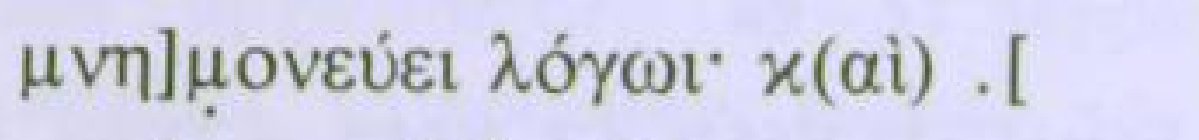 & 10 & \\
\hline$\varepsilon$ & $r$ & 10 & 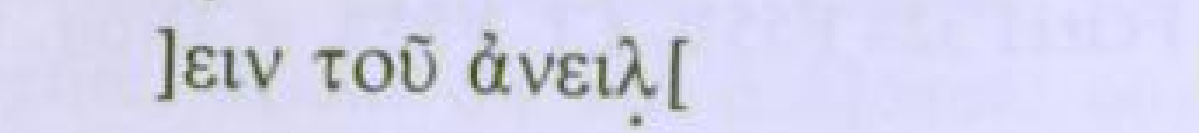 & 14 & \\
\hline
\end{tabular}




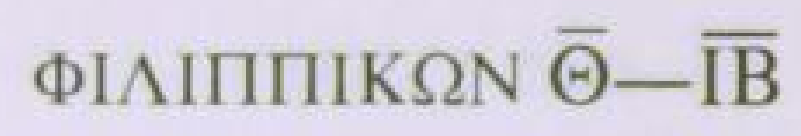

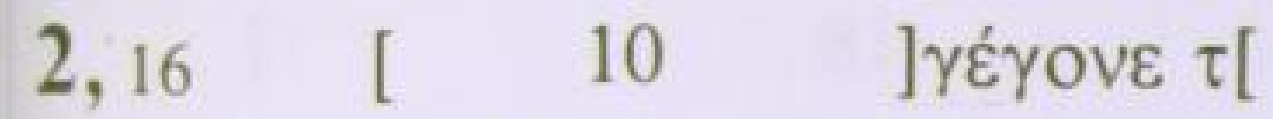

$\begin{array}{lll}\alpha \nu \alpha[ & 14 & \text { ].. [ } \\ \varphi \omega \vee[ & 14 & \text { ].v.[ }\end{array}$

$\grave{\alpha} \theta \rho \cos \alpha v\left[\begin{array}{lll}\tau & 3 & ] \lambda[\ldots] \ldots[\end{array}\right.$

$\begin{array}{ll}18 & ] \\ 16 & \text { ] } \\ 16 & \text { ] } \\ 18 & \text { ] }\end{array}$

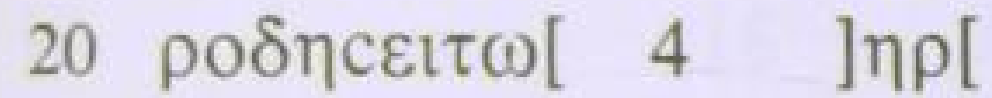
$\alpha \tau \alpha \mathrm{Ic} .$. . С $\chi \cup \theta \tilde{\omega} \nu \mu \varepsilon .[$ 20

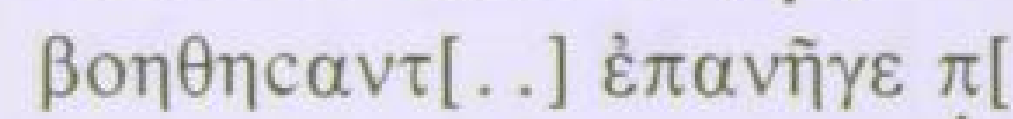
20 $12 \pi \varepsilon-]$

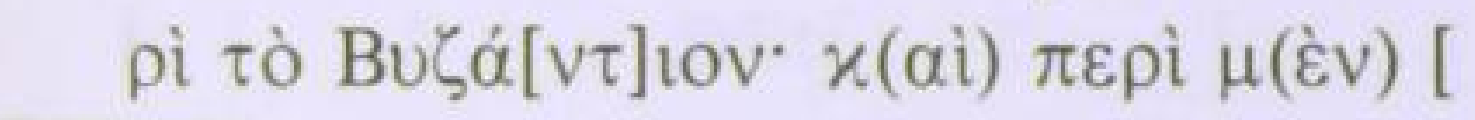
15

\begin{tabular}{|c|c|c|c|}
\hline $\operatorname{cetv}[$ & 6 & ]. $v \omega \cdot \operatorname{cu\mu \beta ou[}$ & 15 \\
\hline$c[$ & 6 & ]c $\alpha \cup ่ \tau o \tilde{c} \pi \circ \lambda[$ & 15 \\
\hline[ & 8 & ]$\mu(\grave{\varepsilon v}) x ı v \delta[u] v \varepsilon \cup o v[$ & 15 \\
\hline [ & 8 & 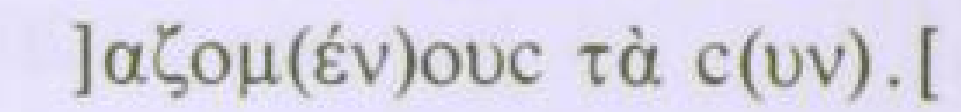 & 12 \\
\hline[ & 6 & 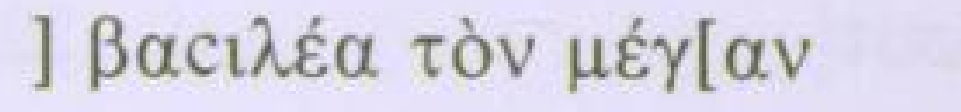 & 10 \\
\hline
\end{tabular}

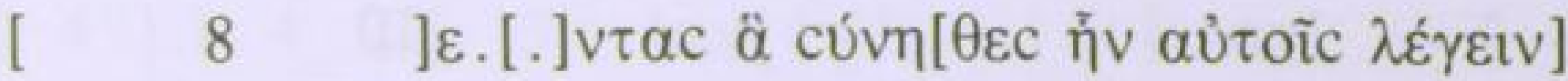

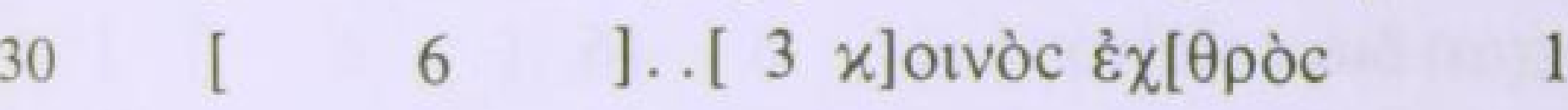

[ 10

$\left.{ }^{ } \mathrm{E} \lambda\right] \lambda \alpha \dot{\alpha} \alpha[$

10

14 ]

\section{$32-54$ perierunt}

55

]

Jacıv

8 ] $\tau$ ou[

]. .


$\begin{array}{rllc}2,58 & {[} & 10 & ] . \alpha c \tau \alpha[ \\ & {[} & 12 & ] . \varepsilon o \ldots[ \\ 60 & {[} & 14 & ] \psi[\ldots] \omega \mathrm{L}[\end{array}$

$\begin{array}{ll}18 & ] \\ 16 & ] \\ 14 & ]\end{array}$

$61-63$ perierunt

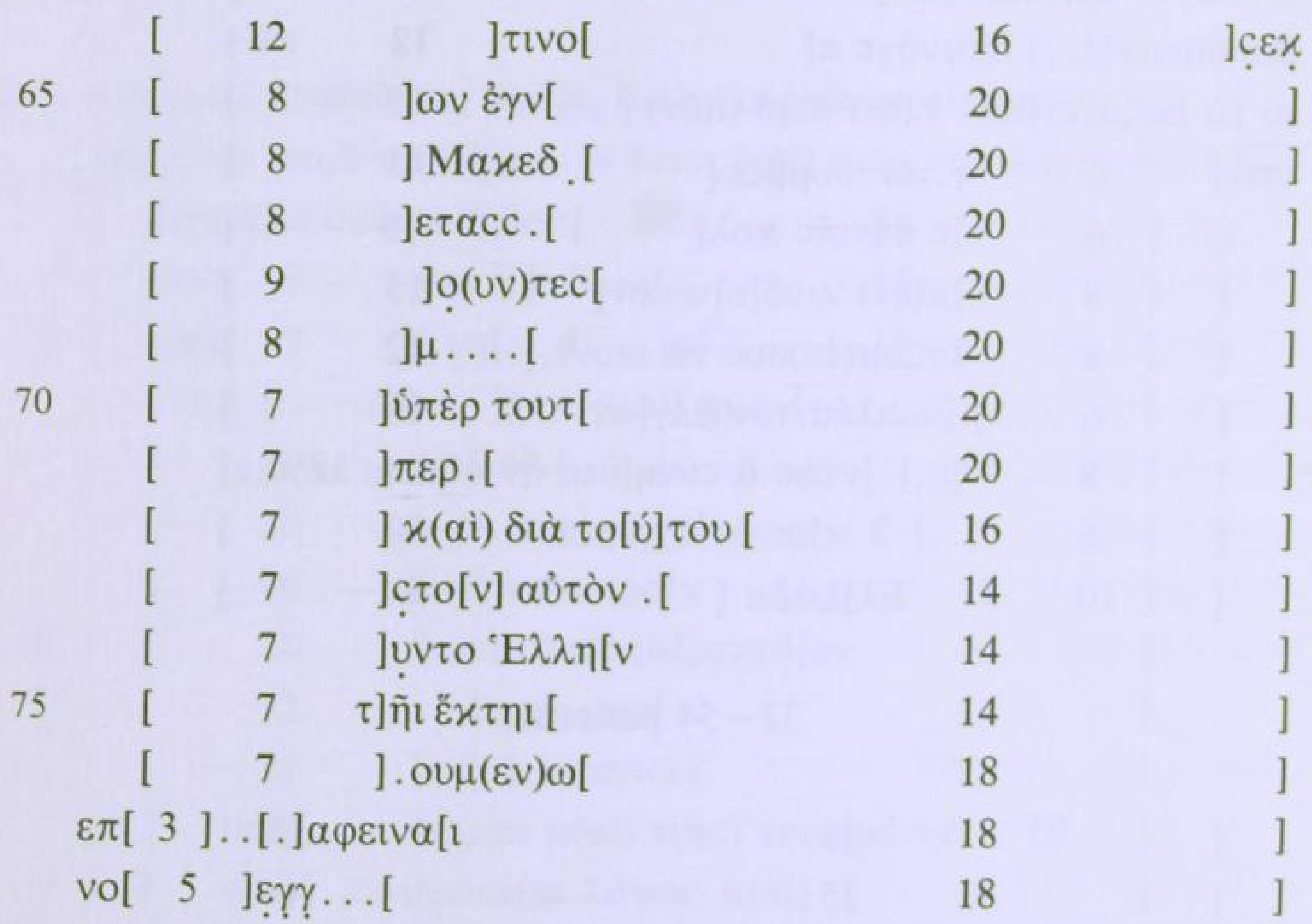




$$
\text { ФІАІППІК } \Omega \mathrm{N} \bar{\Theta}-\overline{\mathrm{IB}}
$$

Col. 3

$1-18$ perierunt integri cum titulo






\begin{tabular}{|c|c|c|}
\hline 3,41 & 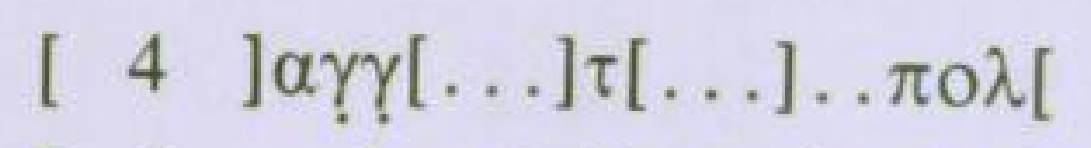 & 16 \\
\hline & 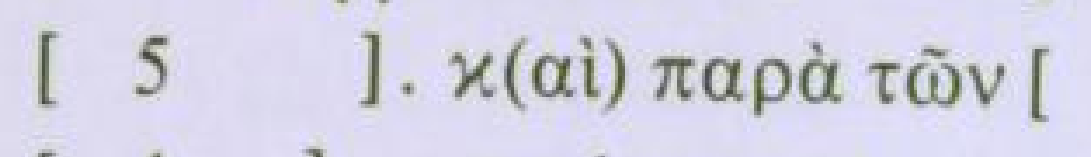 & 20 \\
\hline & 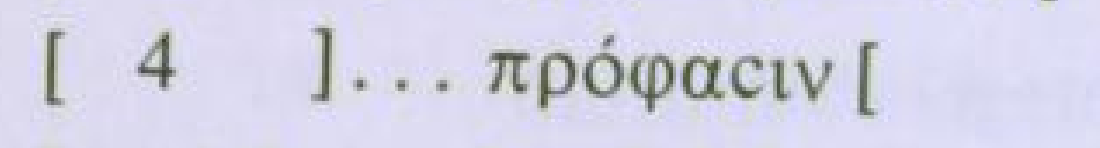 & 20 \\
\hline & {$\left[\begin{array}{lll} & 8 & ] \tau \alpha_{l}[.] \varepsilon[\end{array}\right.$} & 20 \\
\hline 45 & {$\left[\begin{array}{lll}1 & 7 & ] \ldots[\end{array}\right.$} & 20 \\
\hline & {$[\ldots] \ldots \ldots[\ldots] \cdot \varepsilon \cdot[$} & 22 \\
\hline & [.]. $\varepsilon \theta \omega \tau o .[$ & 24 \\
\hline & {$[\ldots] \cdot \mu(\grave{\varepsilon} v)[o] \cup \tilde{v} . . .[$} & 24 \\
\hline & {$\left[\begin{array}{ll}5 & 5\end{array}\right] \varepsilon v[$} & 24 \\
\hline 50 & {$\left[\begin{array}{lll} & 6 & ] \alpha \mathrm{l}\end{array}\right.$} & 24 \\
\hline & {$\left[\begin{array}{lll}{[} & 6 & ]\end{array}\right] \cdot \tau \eta \cdot[$} & 24 \\
\hline & {$\left[\begin{array}{ll}3 & 3\end{array}\right] \tau \alpha \theta \eta$. [ } & 24 \\
\hline & [ 3 ] $] \dot{\delta}(\grave{\imath}) \pi \rho o[$ & 24 \\
\hline & {$[.] \alpha \pi \alpha v \tau \alpha[$} & 24 \\
\hline 55 & {$[..] \mathrm{o}(\cup v)[..] \mu .[$} & 24 \\
\hline & $\pi \alpha \rho \alpha \ldots v \varepsilon .[$ & 24 \\
\hline & $\tau$ touc. $\alpha \ldots[$ & 26 \\
\hline & $\tau \grave{\eta} v \tau(\tilde{\eta} \mathrm{c})[$ & 28 \\
\hline & $\operatorname{cou}(\tau \omega c)[$ & 28 \\
\hline 60 & $\tau \alpha[.] \tau \ldots[$ & 24 \\
\hline & v. [ & 30 \\
\hline & $\theta \underline{\theta}$ & 30 \\
\hline
\end{tabular}


Col. 4

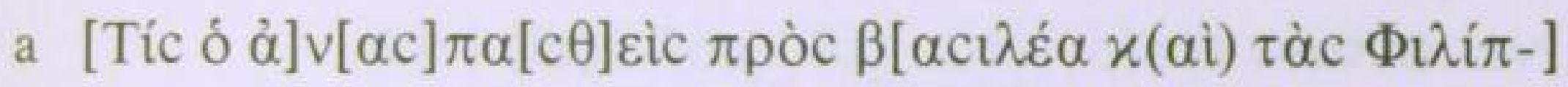

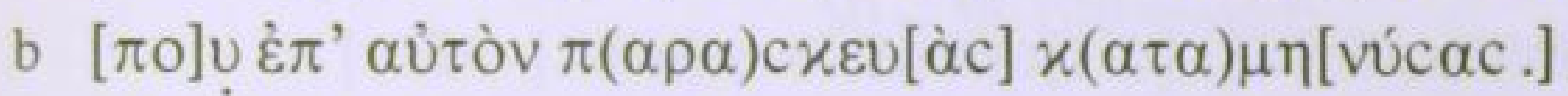

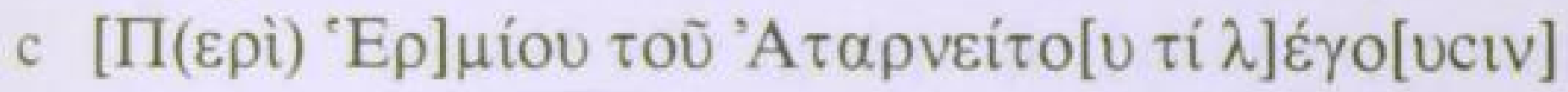

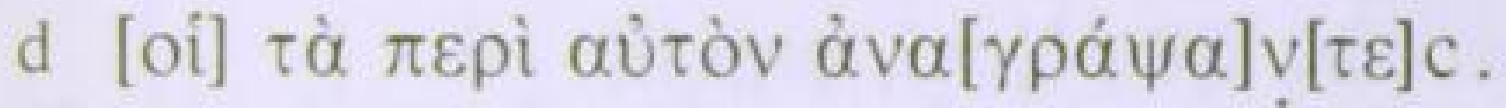

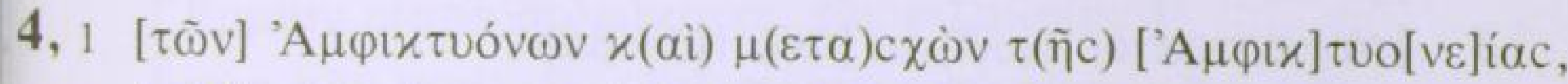

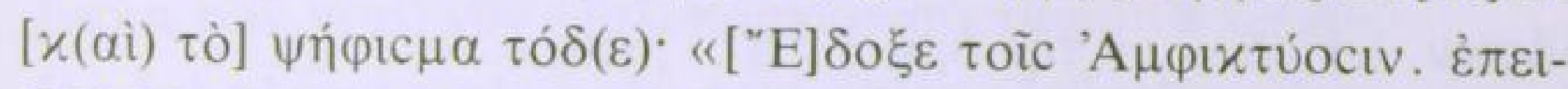

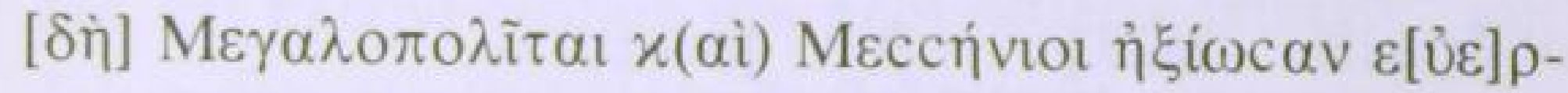

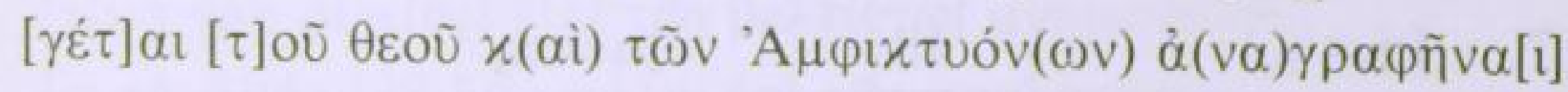
$x(\alpha i)$

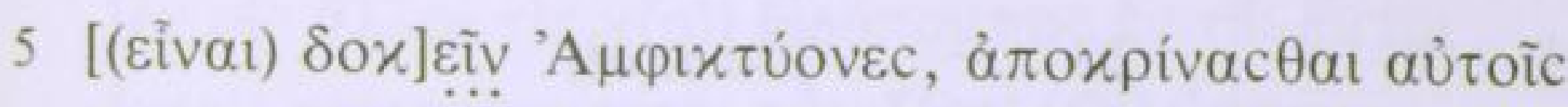

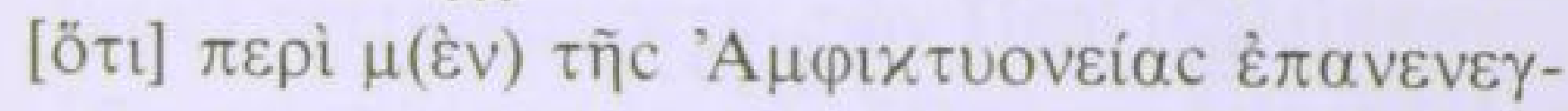
[

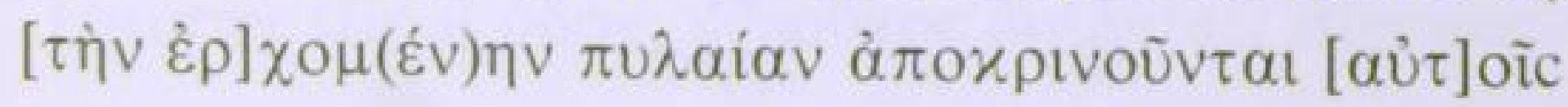

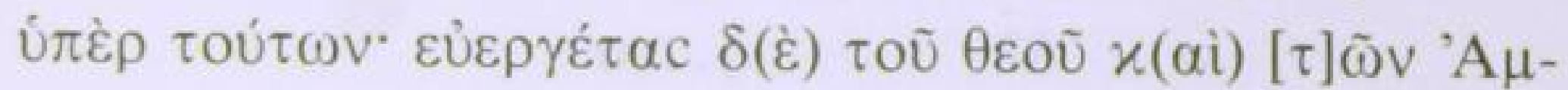




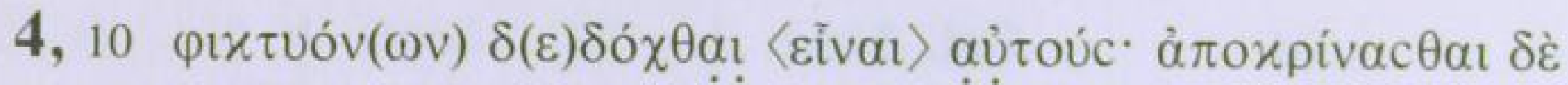

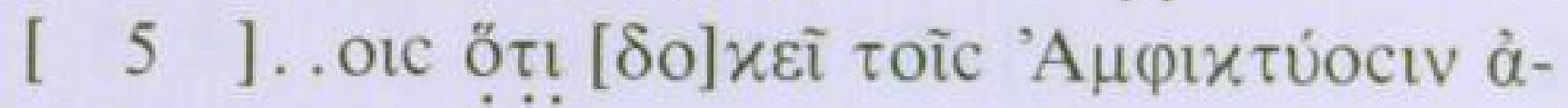

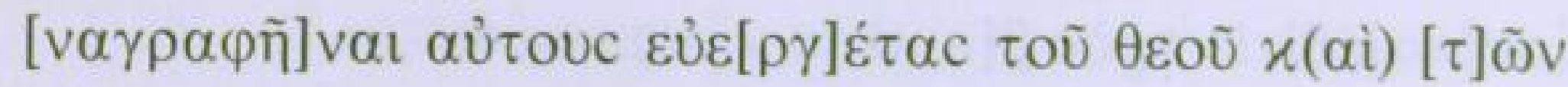

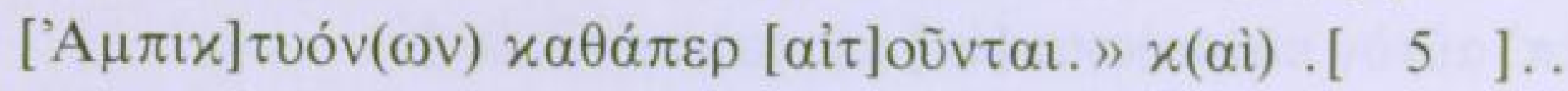

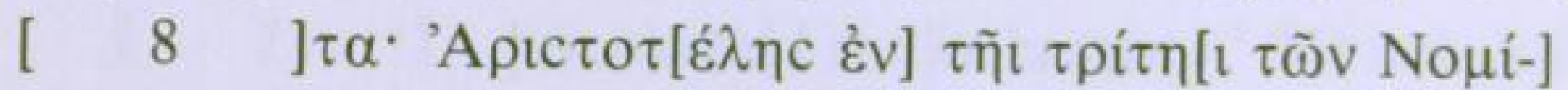

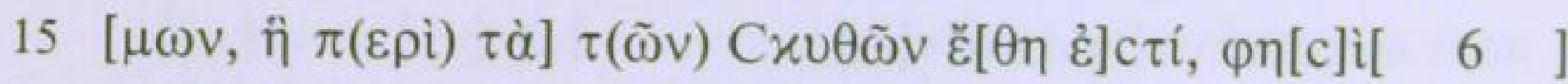

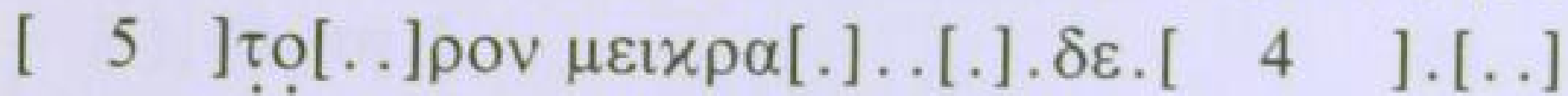
[ 6 ] $[\beta \alpha ́ \rho \omega v \ldots] \ldots \delta \ldots v \tau \tilde{\eta} \mathrm{c} \delta \rho \alpha c[8$ ] $8 \mathrm{c} \alpha \rho \mathrm{s}$

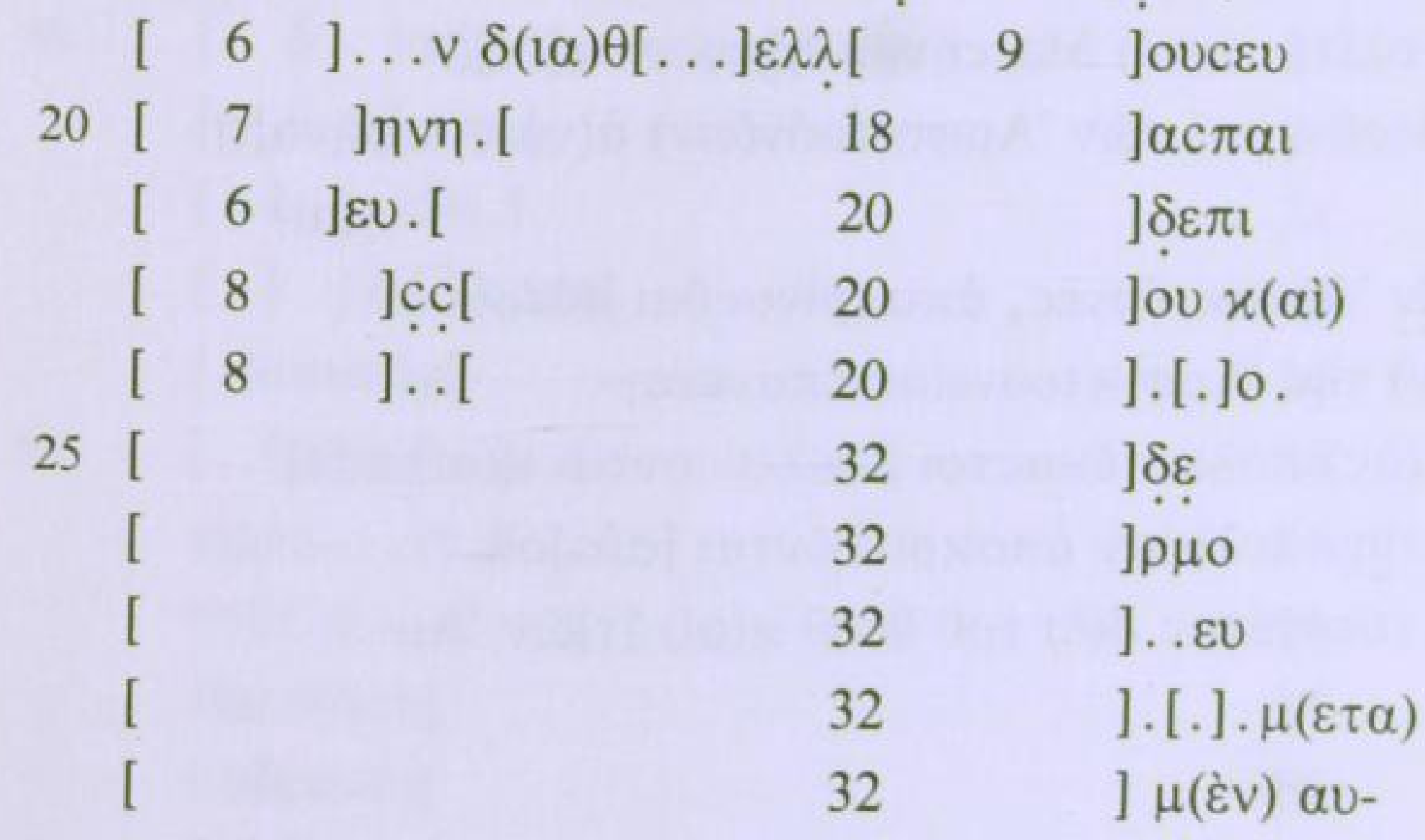

$30-46$ perierunt

47 12

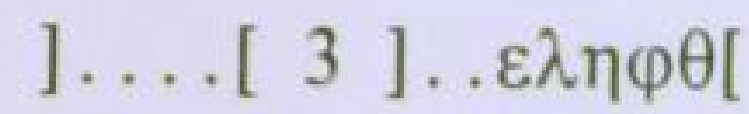
$8 \quad]$ 
4, $48[15 \quad$ lọ̣ ..v

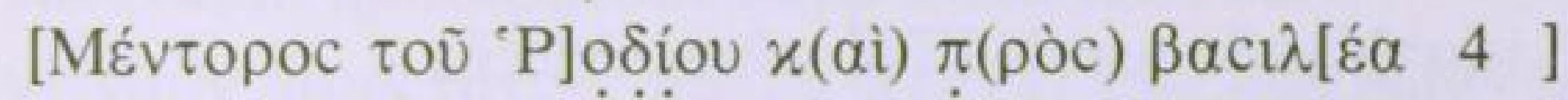

$$
50-58 \text { oblitterati }
$$

[

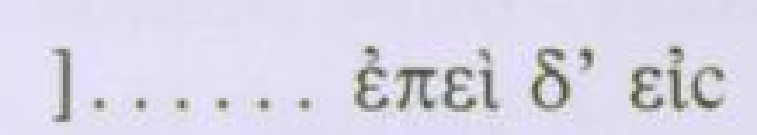

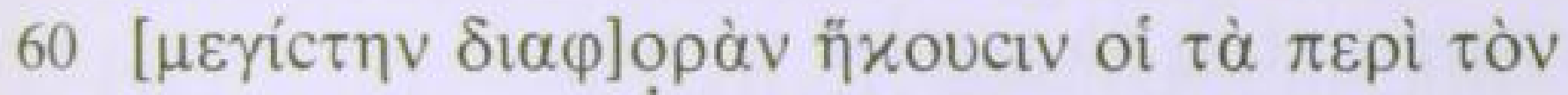

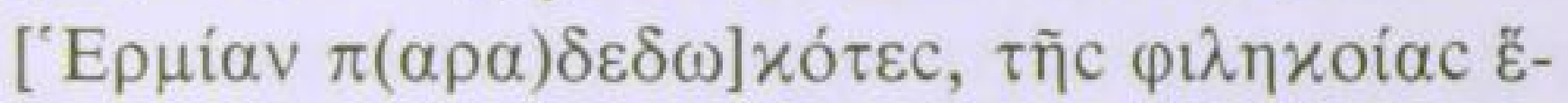

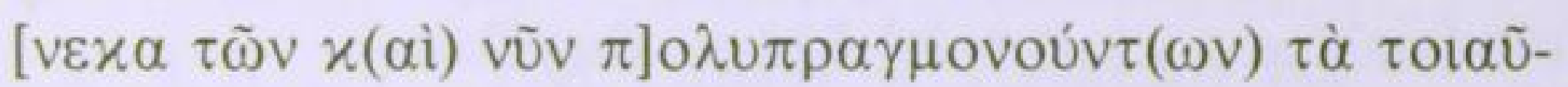

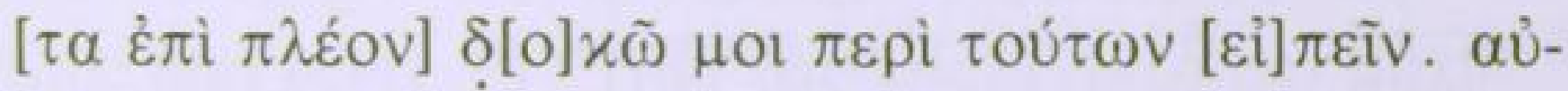

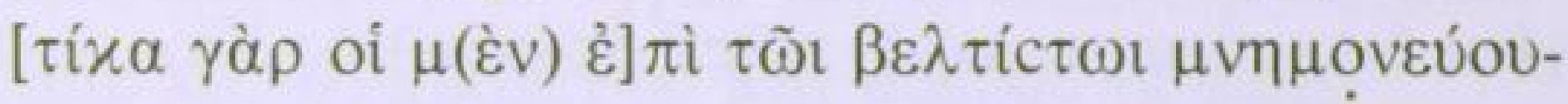

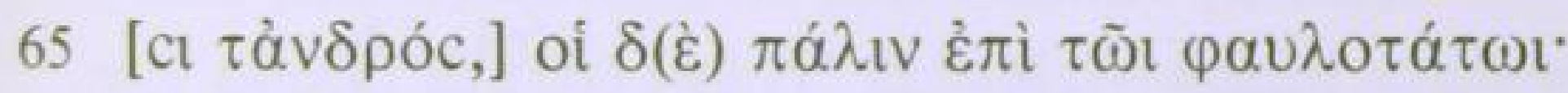

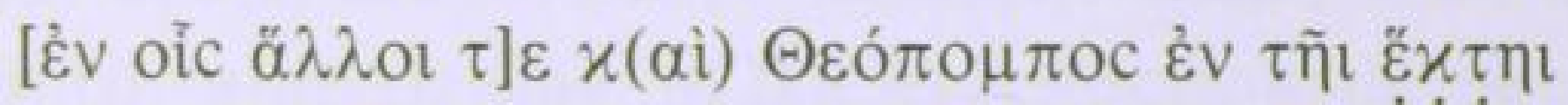
[

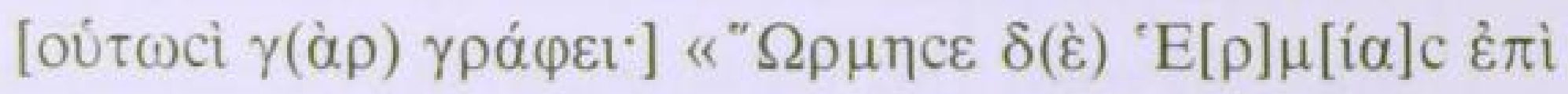

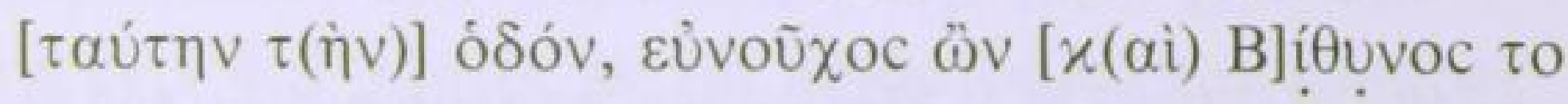




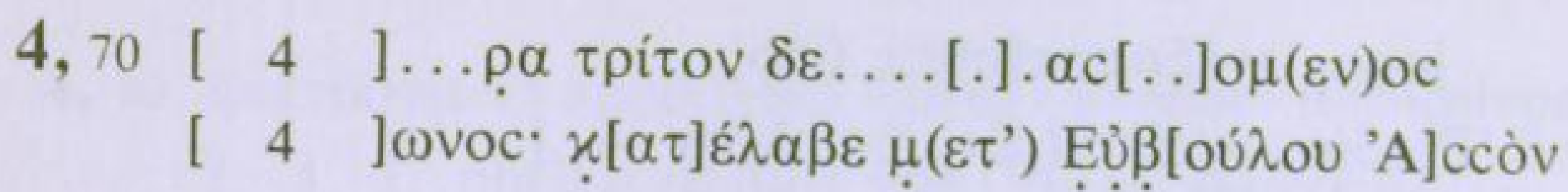

\section{Col. 5 .}

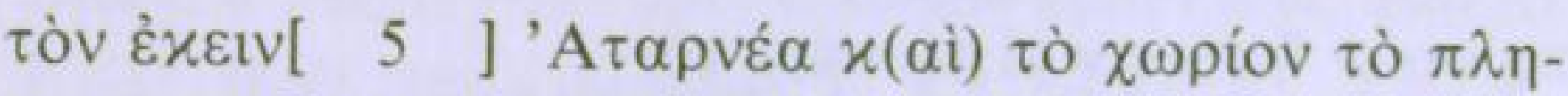

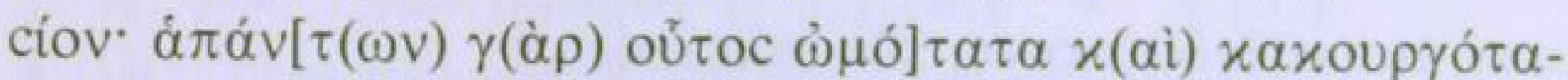

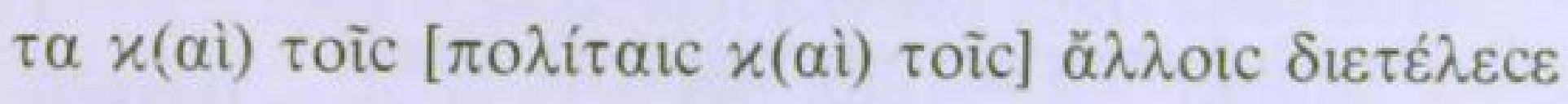

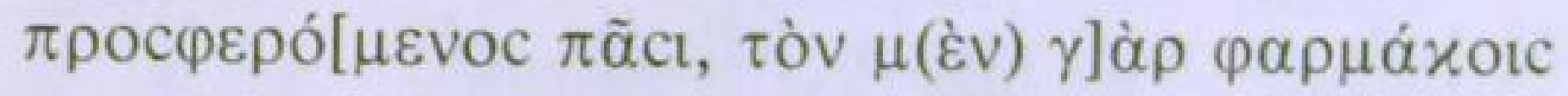

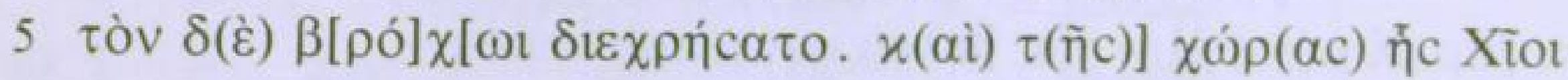
$x(\alpha i) \mathrm{Mt}-$ 


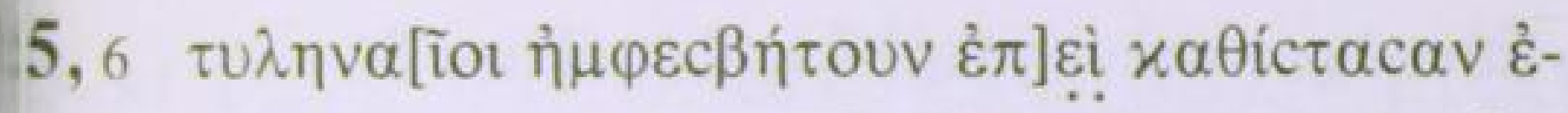

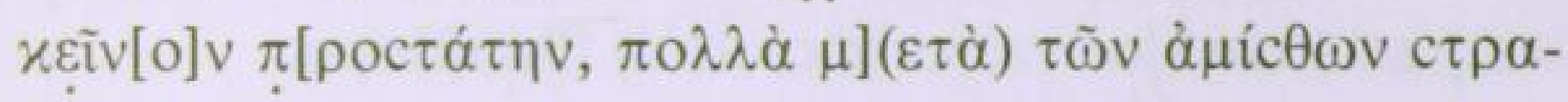

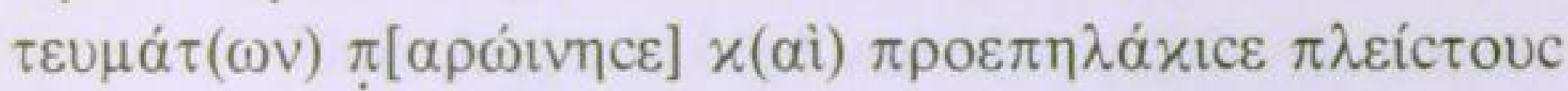

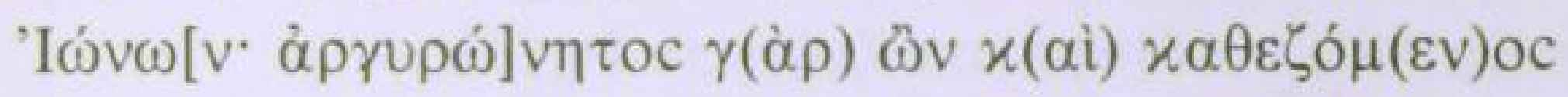

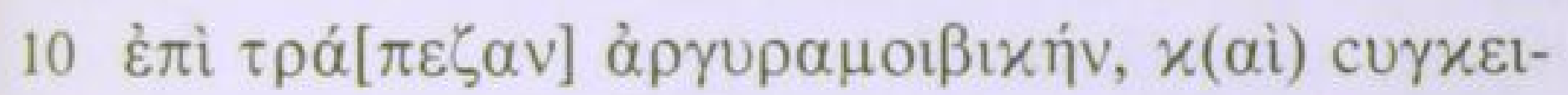

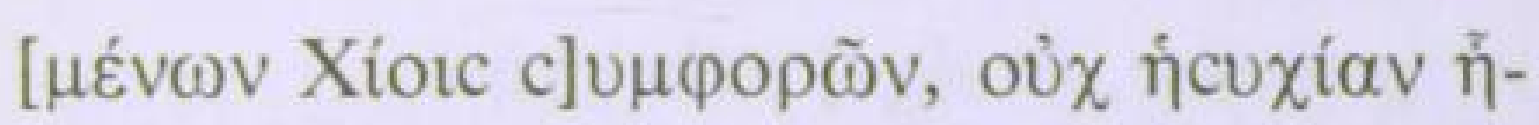

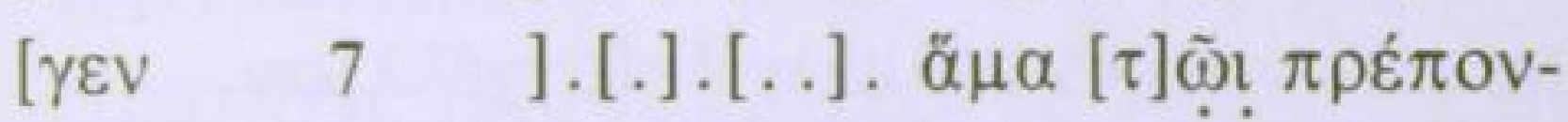
$\left[\begin{array}{lllllll}\tau 1 & 10 & ] \delta[ & 4 & ] c[. .] & \varepsilon & \pi 0 \lambda \lambda \tilde{\omega} \nu \delta \dot{\varepsilon}\end{array}\right.$

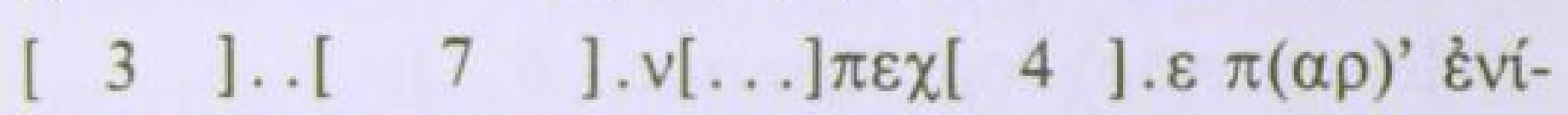

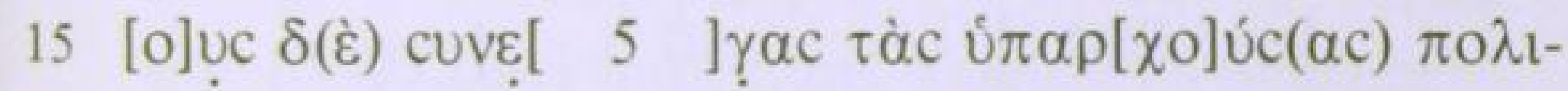

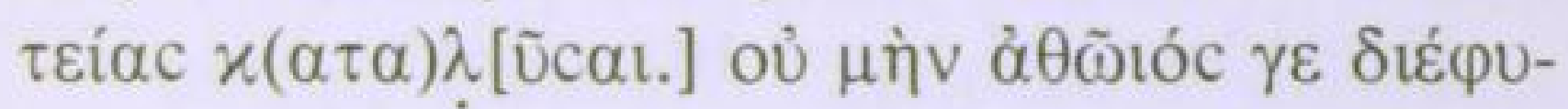

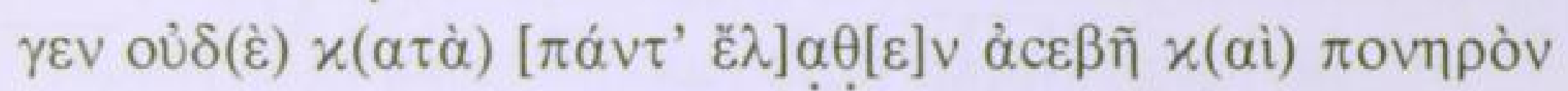

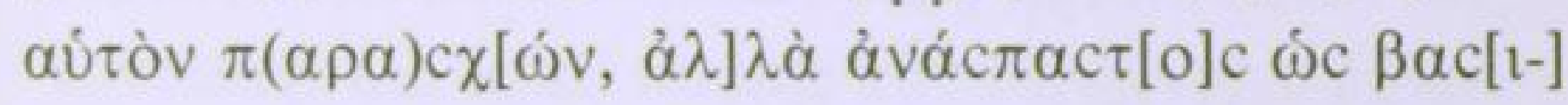

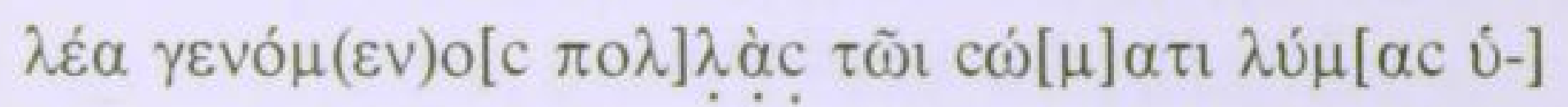

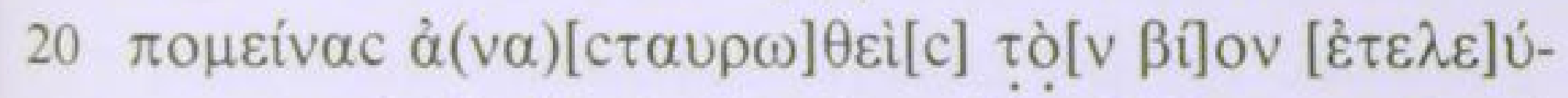

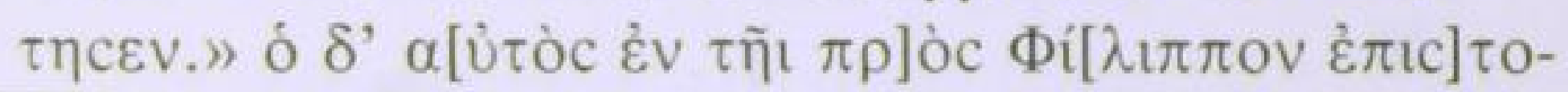

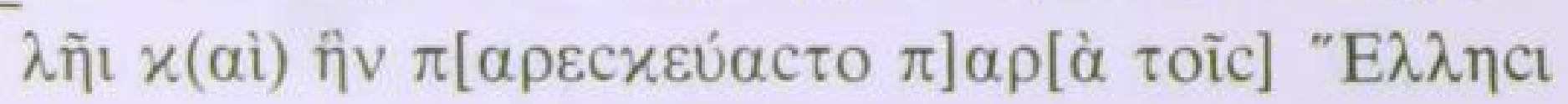




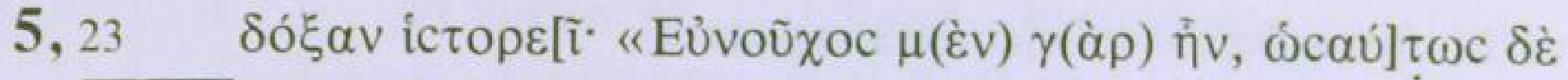

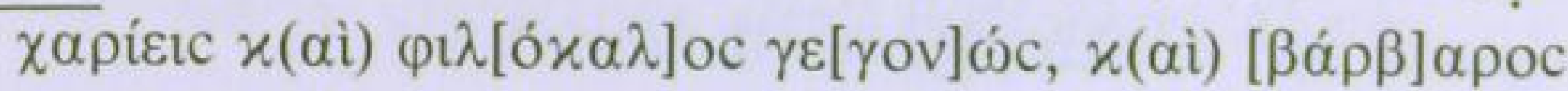

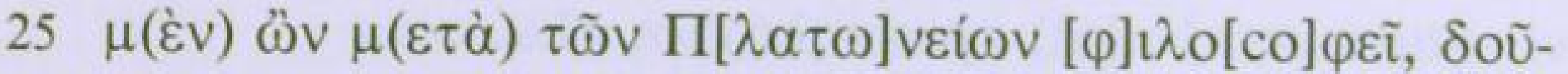

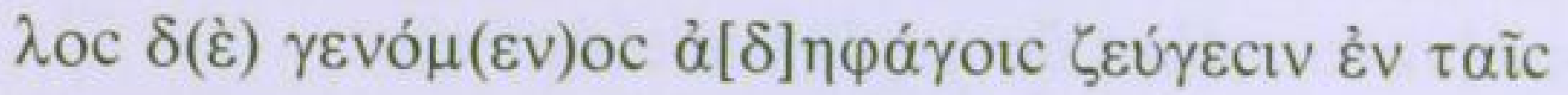

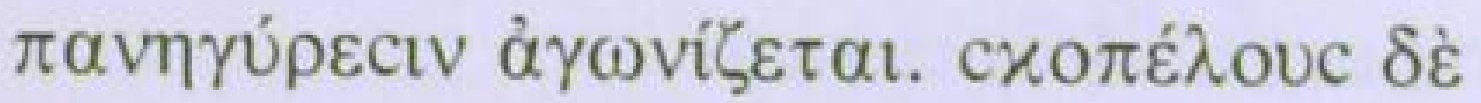

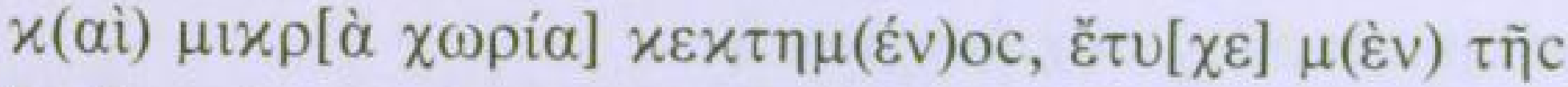

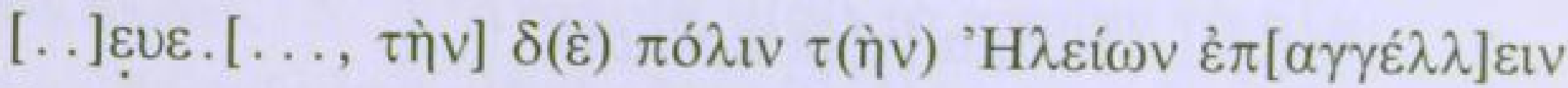

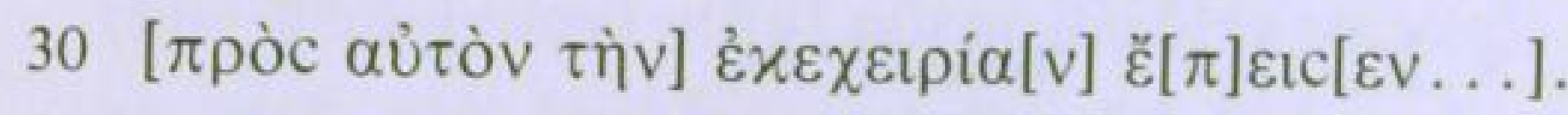

\begin{tabular}{|c|c|}
\hline 10 & 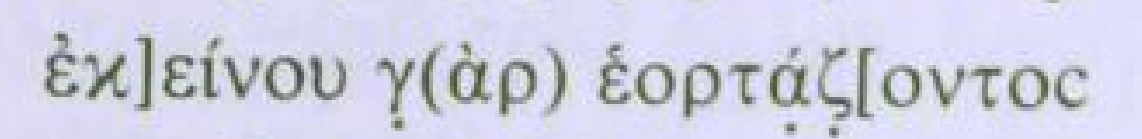 \\
\hline 16 & ]$\omega v \cdot \delta(\varepsilon) \cdot[$ \\
\hline 16 & ]$\delta(\iota \alpha) \lambda \sigma \gamma . . \lambda[$ \\
\hline 17 & ].o[..]. $\mu(\varepsilon v)[$ \\
\hline
\end{tabular}

$35-43$ integri perierunt

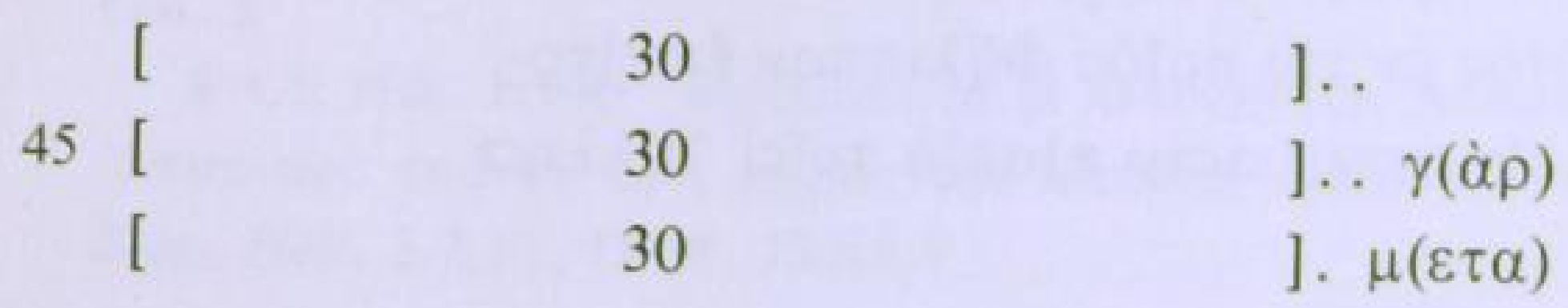


$\begin{array}{rrr}5,47 & {[} & 28 \\ & {[} & 24 \\ & {[} & 24 \\ 50 & {[} & 24 \\ & {[} & 20\end{array}$

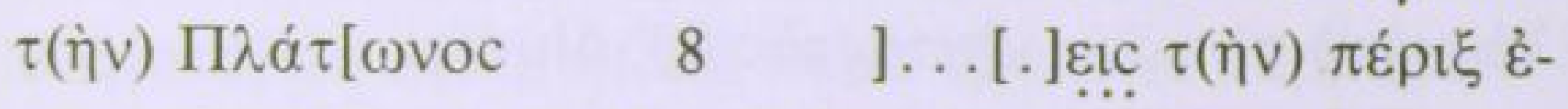

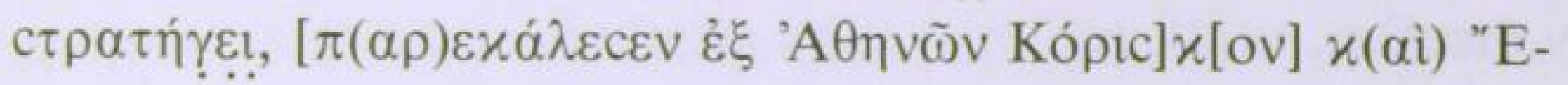

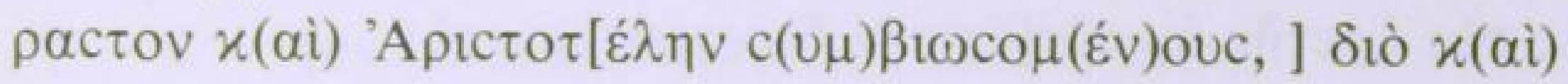

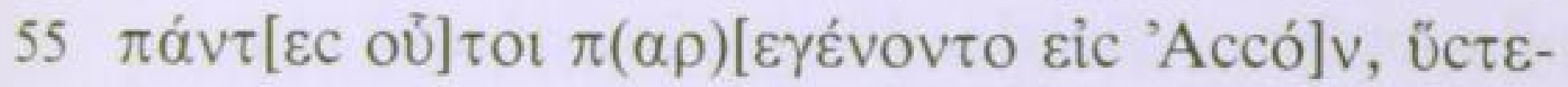

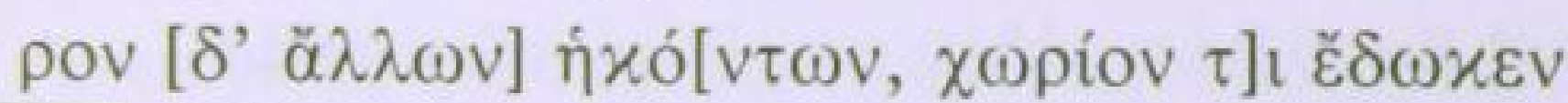

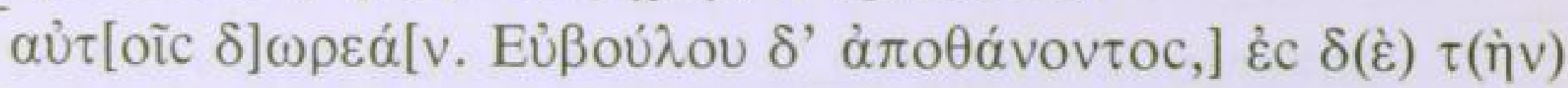

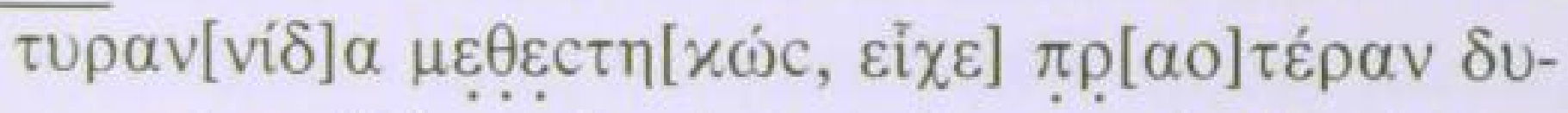

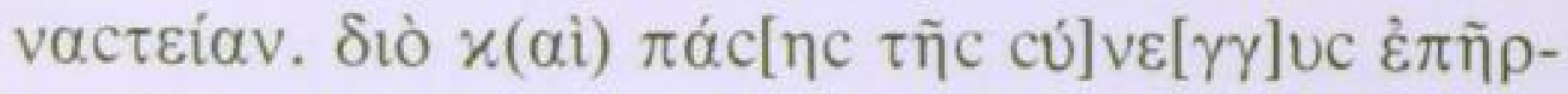

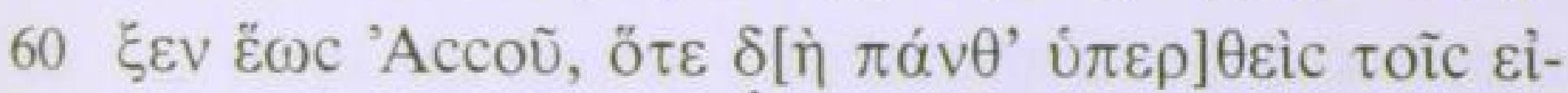

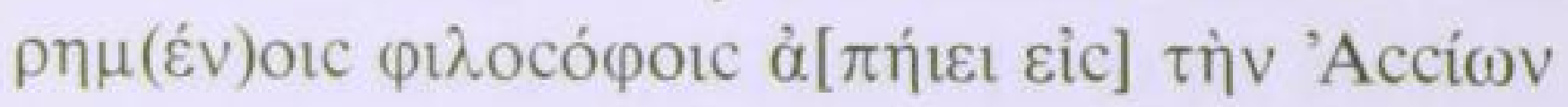




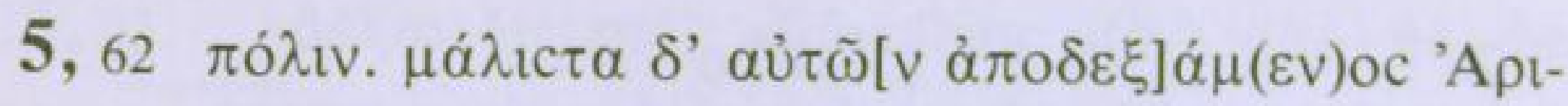

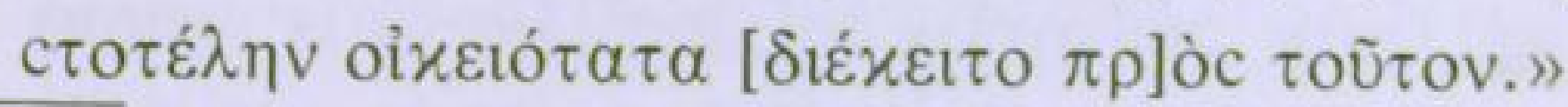

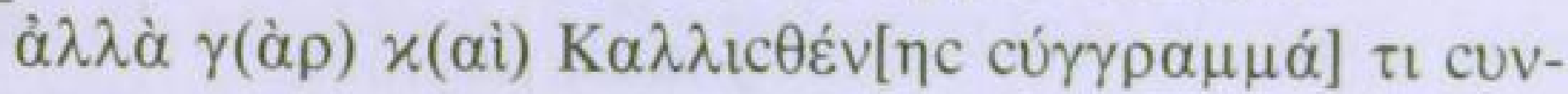

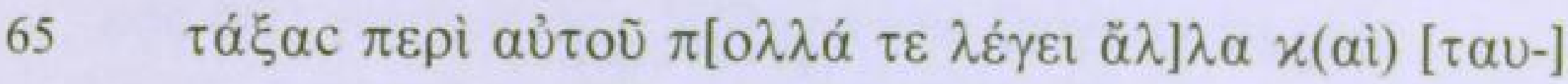

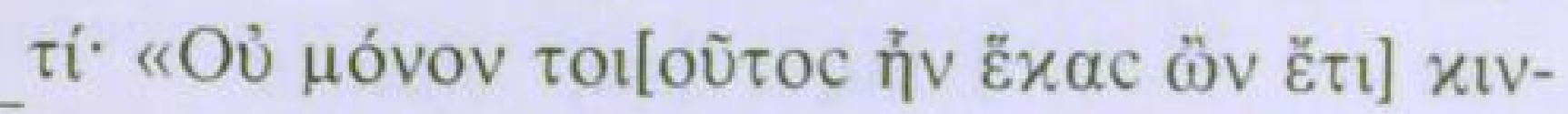

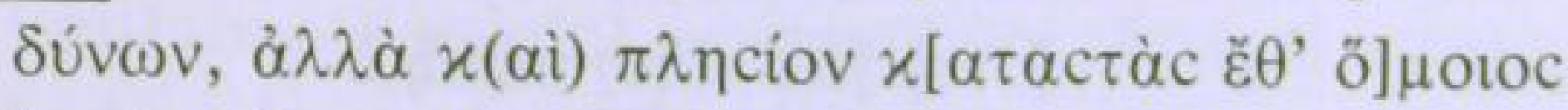

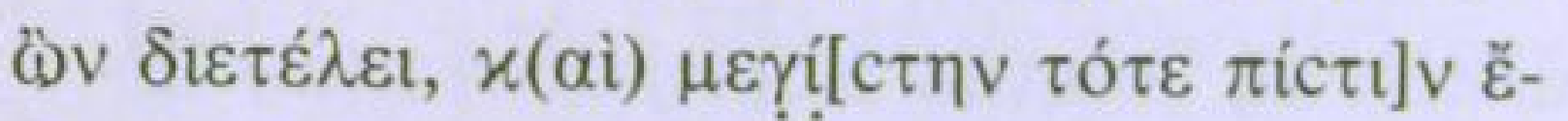

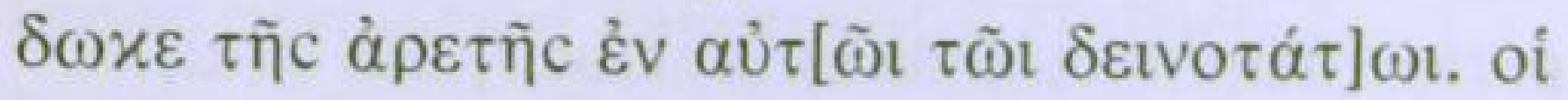

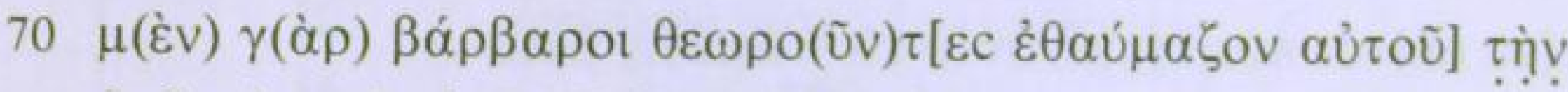

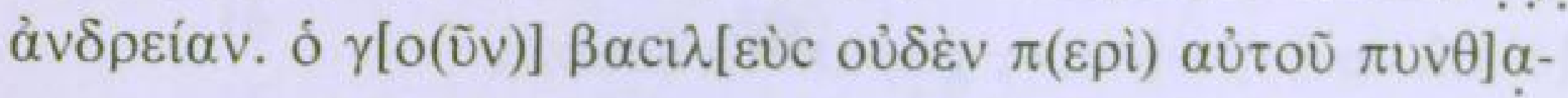

Col. 6

a 'Y $\pi[\varepsilon \rho \beta] \alpha \dot{\tau} \tau \cup \varphi \rho \alpha \dot{c} \varepsilon \omega[\mathrm{c}] \chi(\alpha \tau \dot{\alpha}) \mathrm{c} \tau \alpha \mathrm{cıc}$.

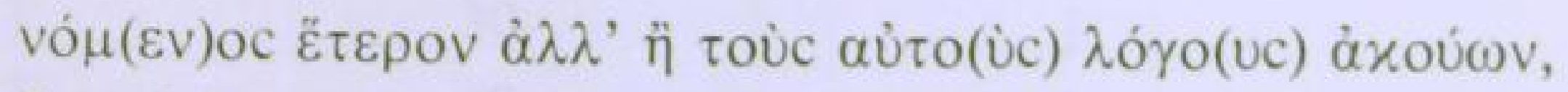

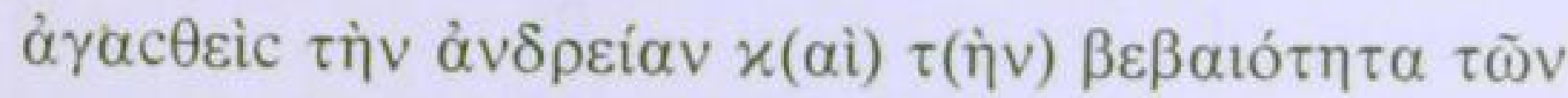

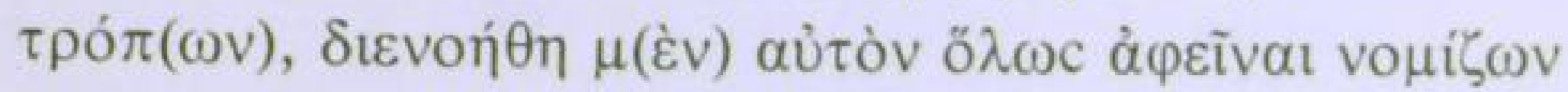

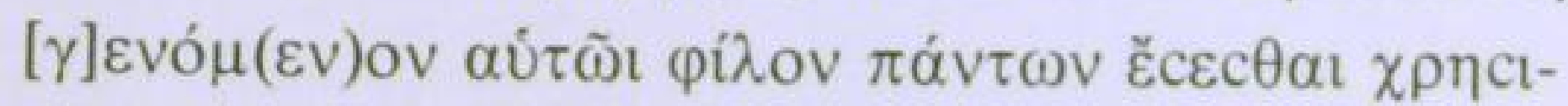

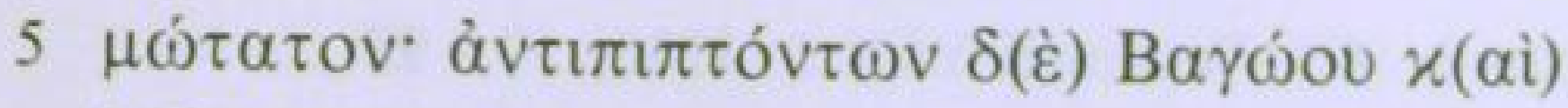

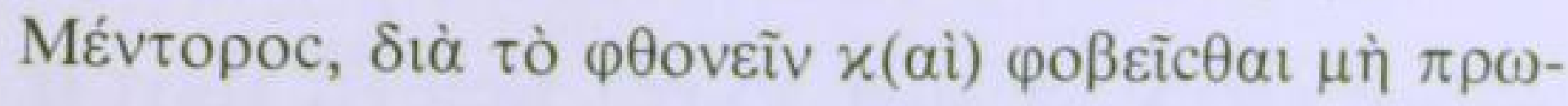




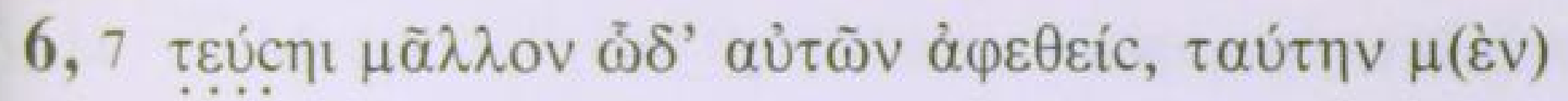

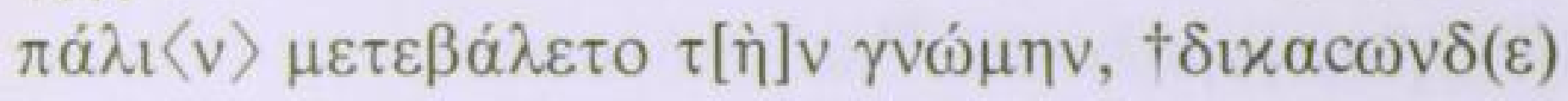

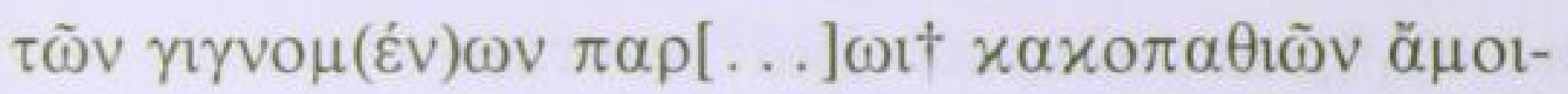

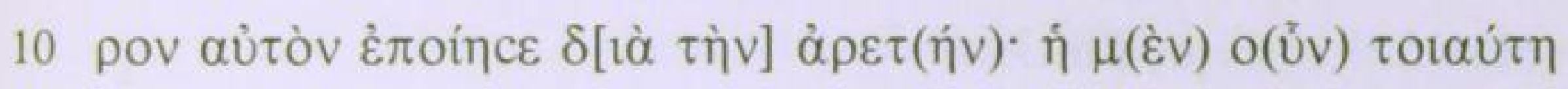

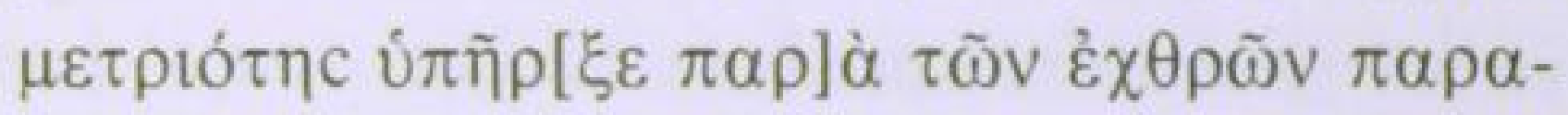

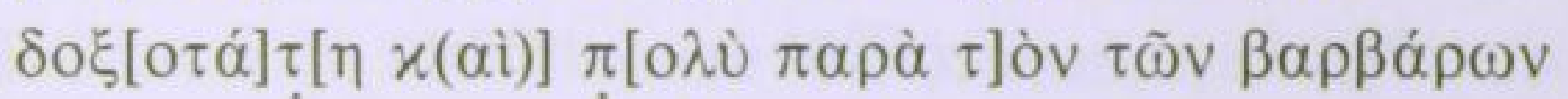

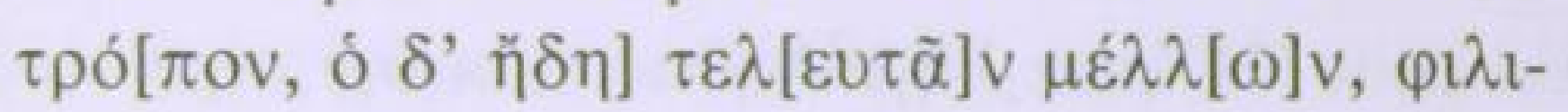

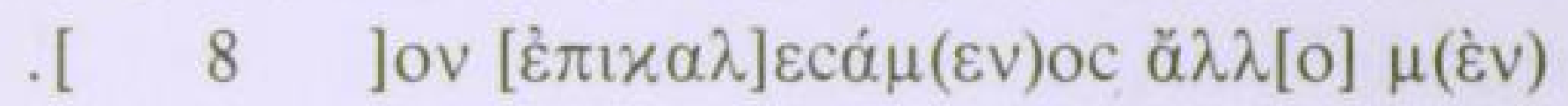

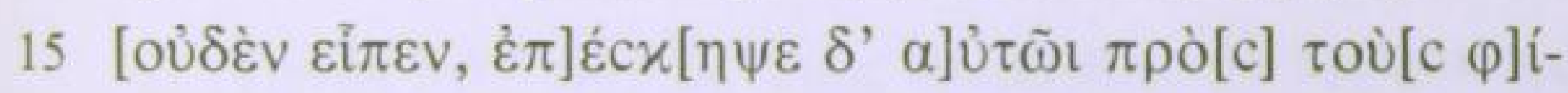

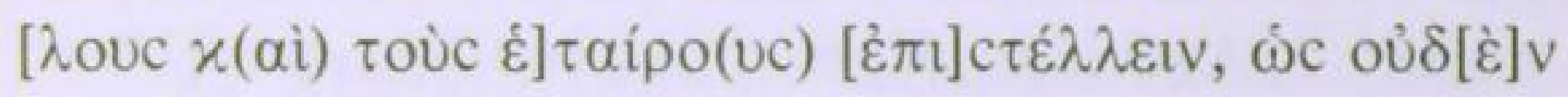

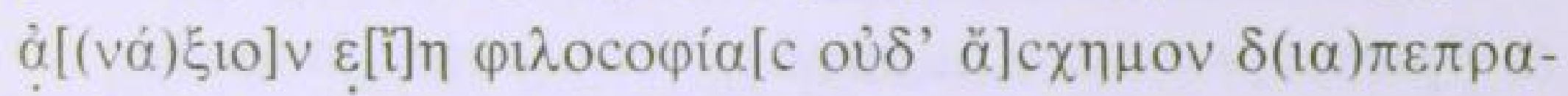
$\gamma \mu[(\dot{\varepsilon} v)]$ oc.» $x(\alpha \hat{i}) \dot{\eta} x \eta \delta \varepsilon i \alpha \delta(\dot{\varepsilon}) \hat{\eta} \pi \rho[o ̀ c] \tau o ̀ v ~ ' A \rho t c \tau o \tau \varepsilon ́ \lambda \eta$

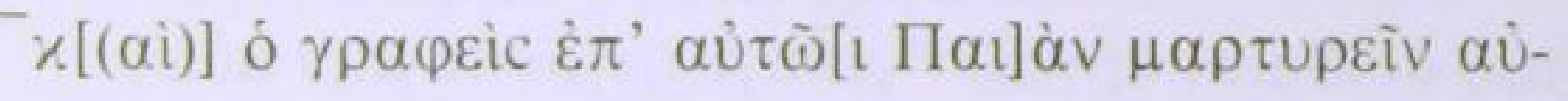

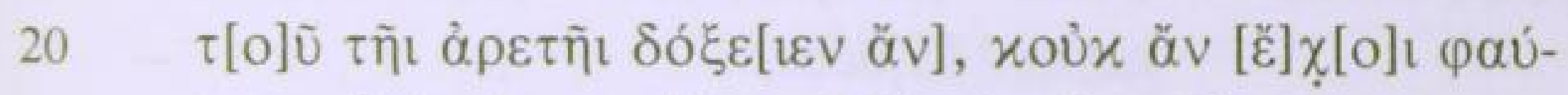

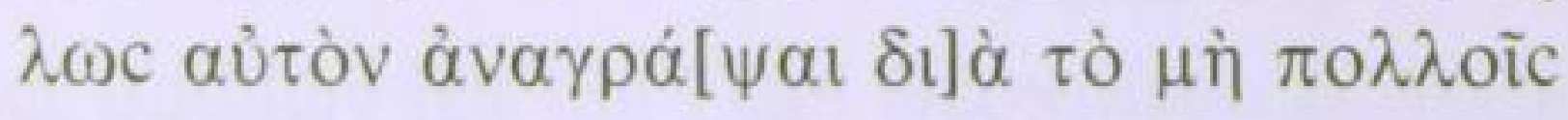

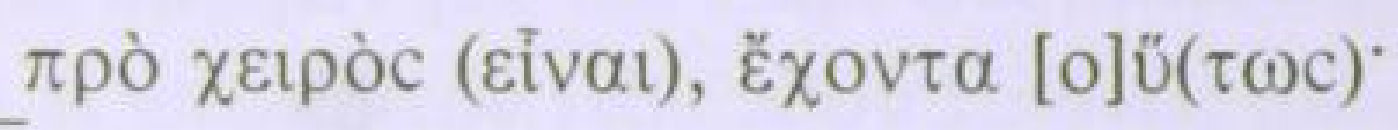

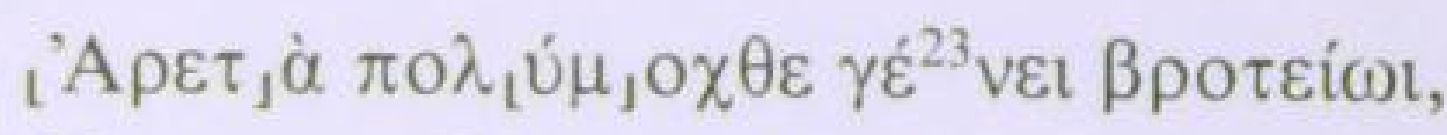
$\theta \dot{\rho} \rho \alpha \mu \alpha{ }_{1} \chi \alpha \dot{\lambda} \lambda l_{1} \mathrm{c} \tau$ ov $\beta i \omega$, 
6, $23 \quad c \tilde{\alpha} c \pi \varepsilon^{24} \rho 1, \pi \alpha \dot{\alpha} \theta \varepsilon v \varepsilon, \mu о \rho \varphi \tilde{\alpha} c$

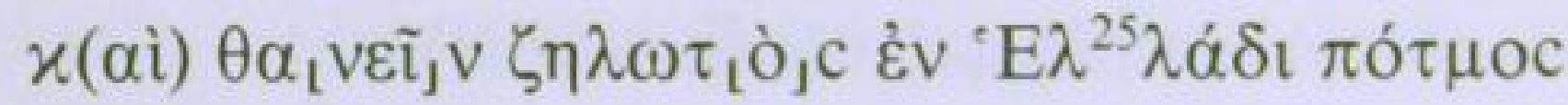

$5 \quad x(\alpha i) \pi$ óvouc $\tau \lambda_{1} \tilde{\eta}_{1} v \alpha \imath \mu \alpha \lambda \varepsilon \rho o u ̀ c ~ \alpha x \alpha^{26} \mu \alpha v \tau \alpha c$.

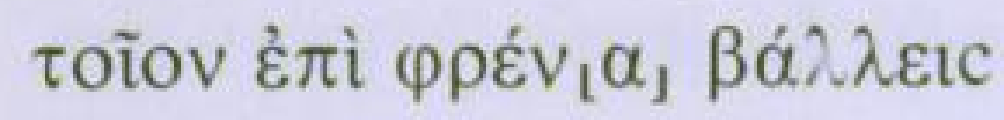

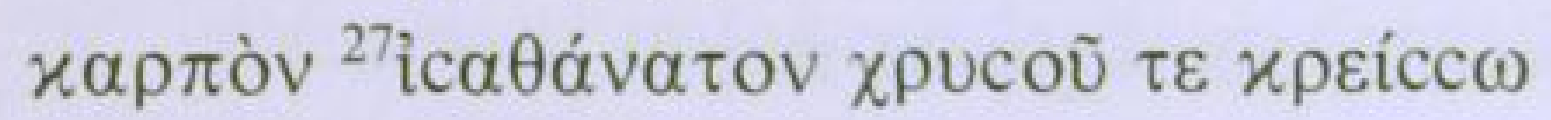

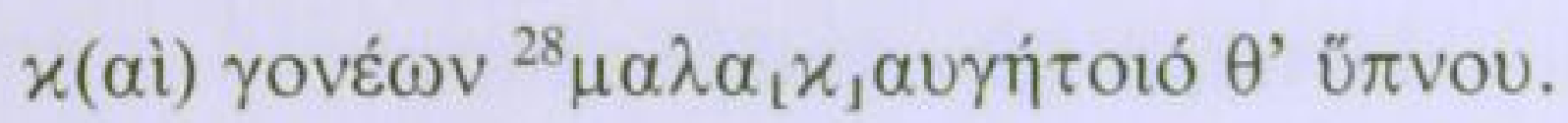

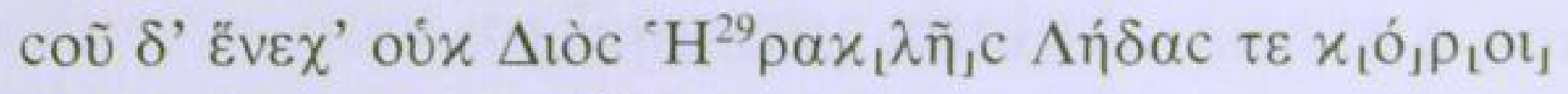

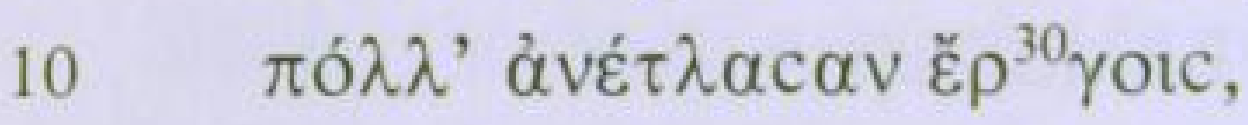

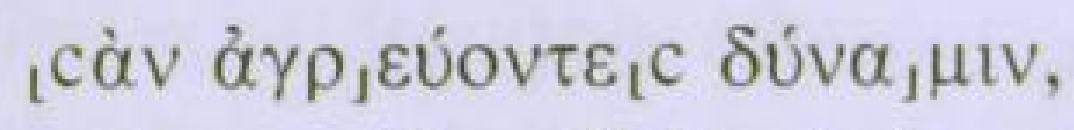

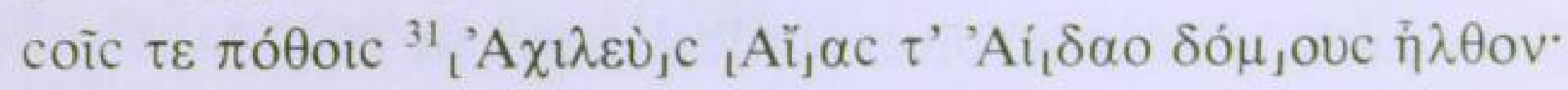

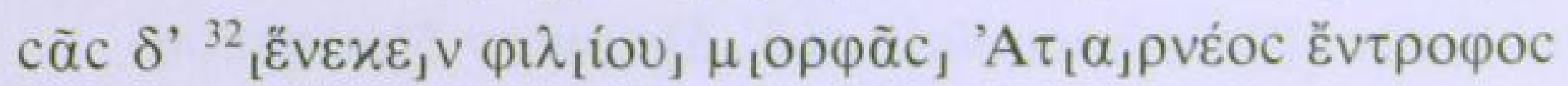

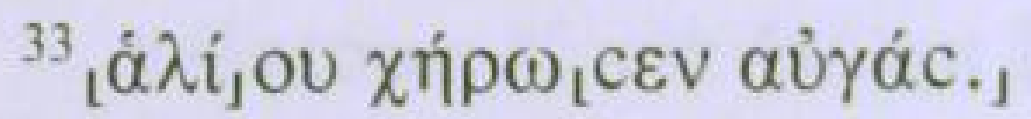

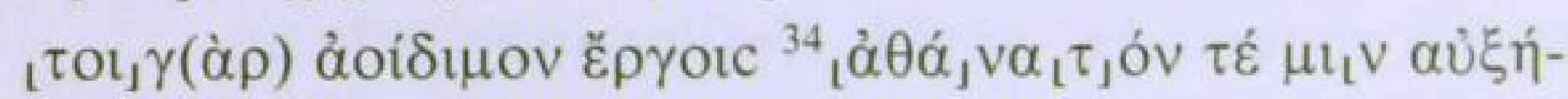
cover $\mathrm{Mo}, \tilde{\mathrm{Uc}} \alpha \mathrm{l}$, 


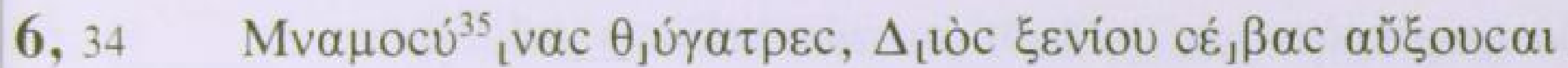
$\varphi \iota^{36}{ }_{1} \lambda i \alpha c \tau_{\jmath} \varepsilon \gamma \varepsilon \dot{\rho} \rho c \beta_{\iota} \varepsilon \beta \alpha$ íov.

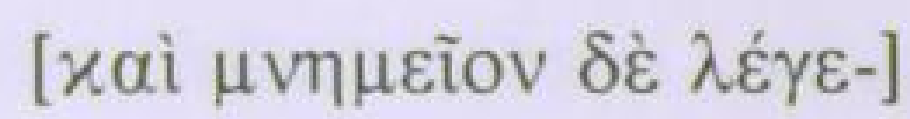

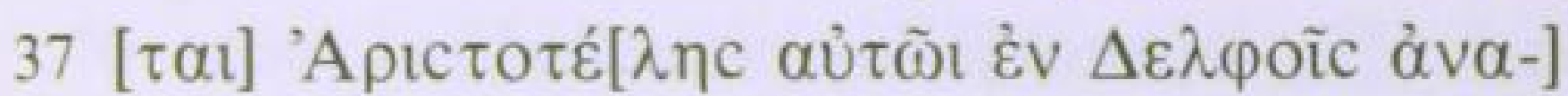

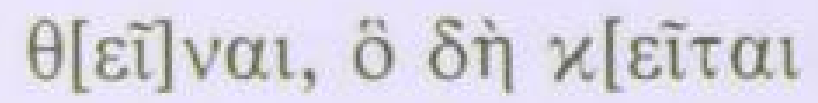
12

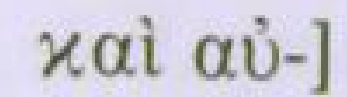

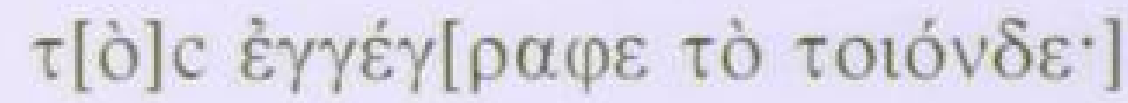

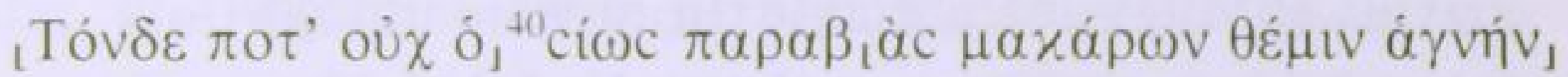

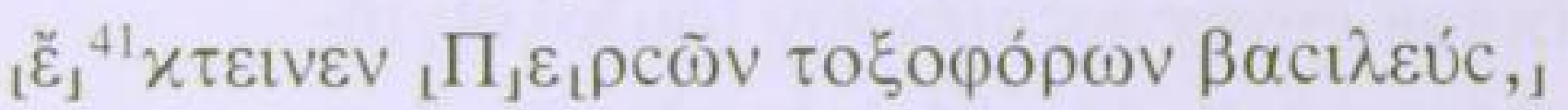

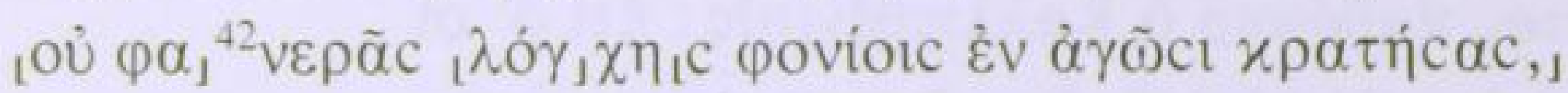

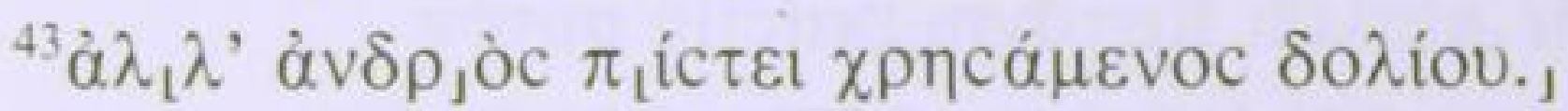

\section{[ $\pi \rho$ òc őv]}



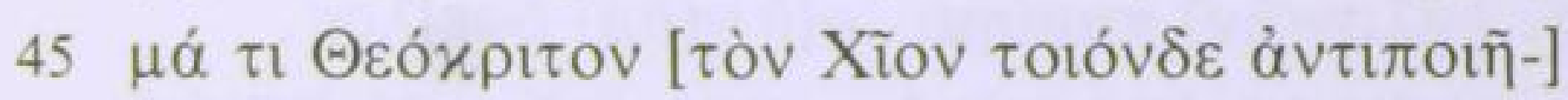
$\mathrm{c} \alpha \mathrm{I}^{\circ}$

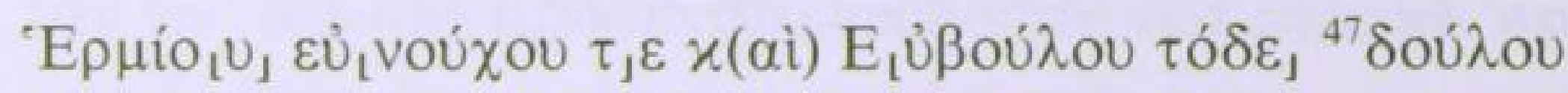

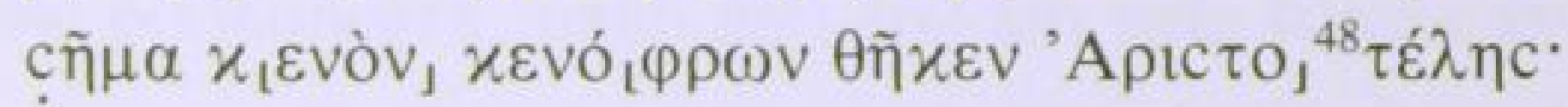




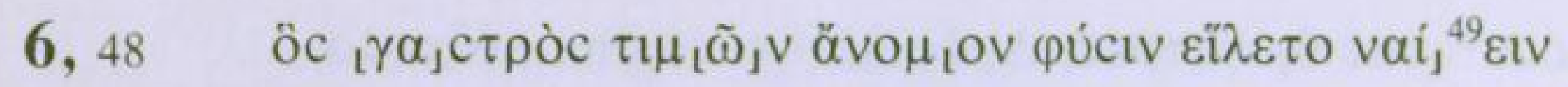

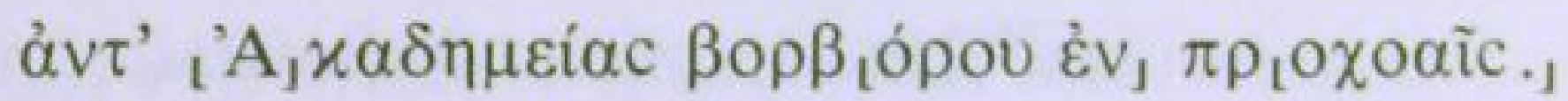

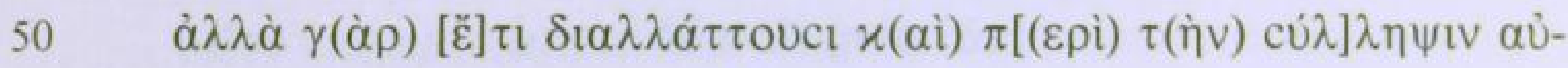

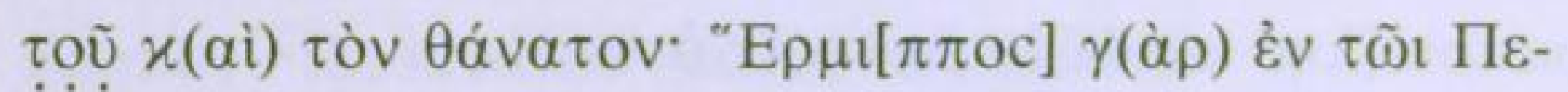

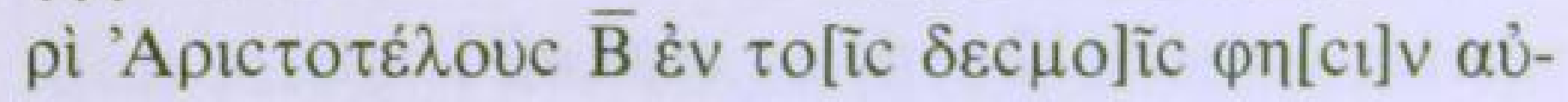

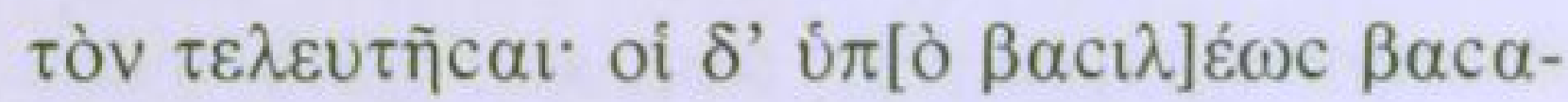
[vic] $\theta \dot{\varepsilon} v \tau \alpha \dot{\alpha}(v \alpha) c \tau \alpha u \rho \omega \theta \tilde{\eta} v \alpha[1, x \alpha \theta] \alpha \dot{\pi} \varepsilon \rho \pi \rho \circ \varepsilon ́ x-$

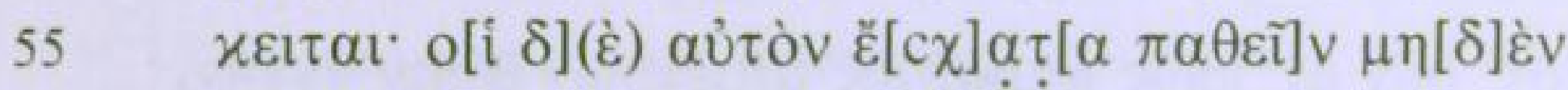

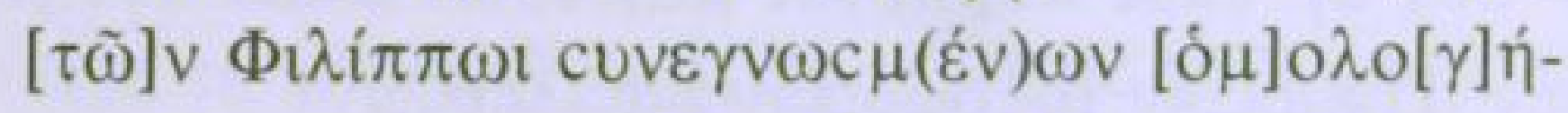

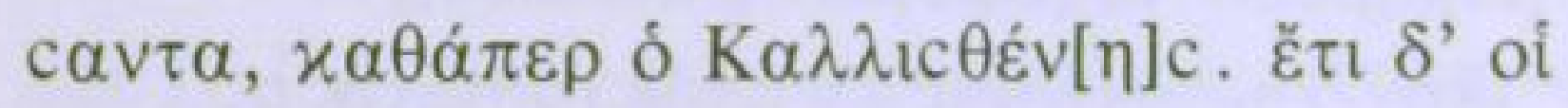

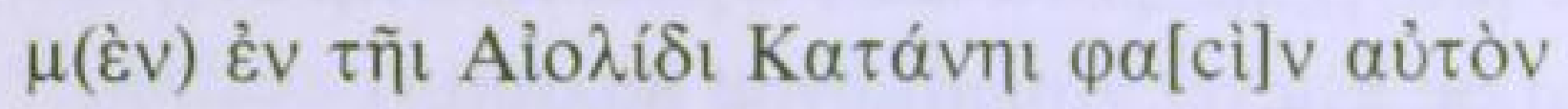

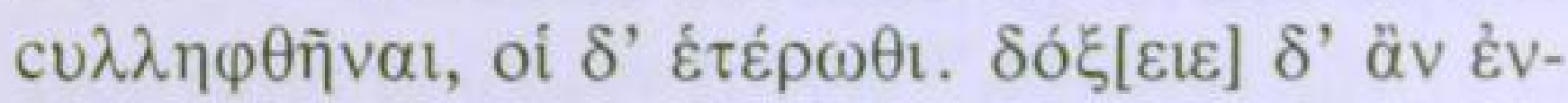

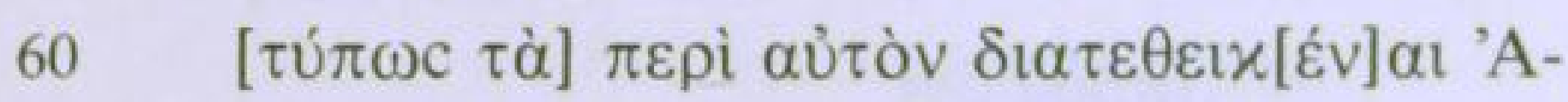

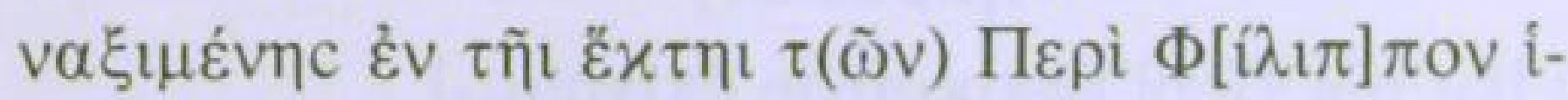

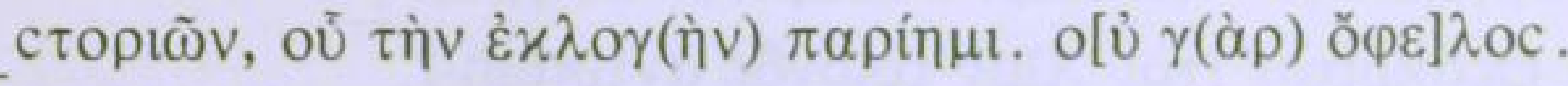

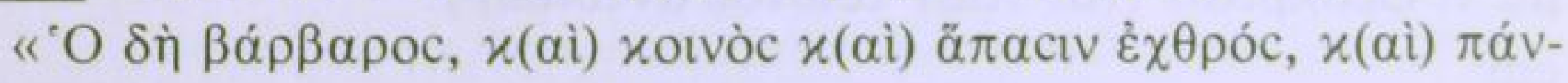

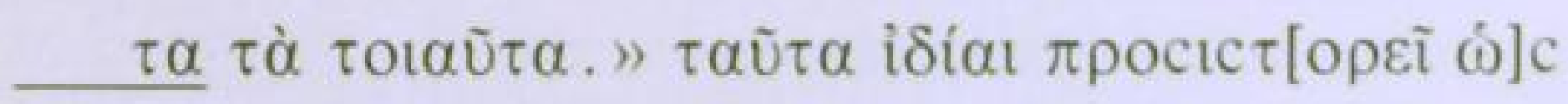

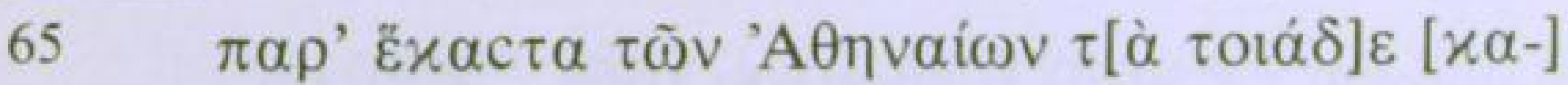




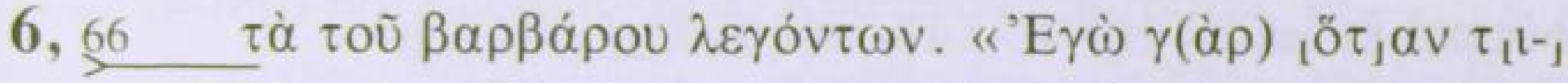

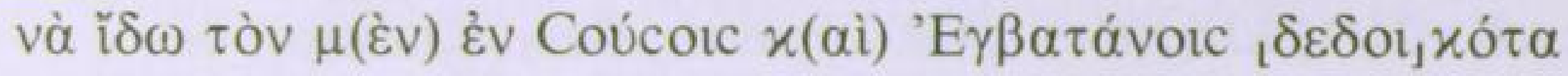

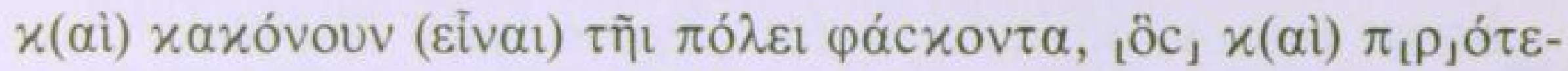
$\rho o v \mathrm{c}(u v) \varepsilon \pi \eta v \omega ́ \rho \theta \omega c \varepsilon \tau \dot{\alpha} \tau(\tilde{\eta} \mathrm{c}) \pi \delta ́ \lambda \varepsilon \omega c \pi \rho_{\mathrm{l}} \dot{\alpha} \gamma \mu_{\mathrm{J}} \alpha_{\mathrm{l}} \tau \alpha_{\mathrm{J}} \chi(\alpha \dot{\mathrm{i}})$

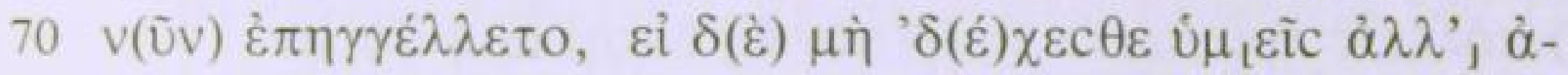

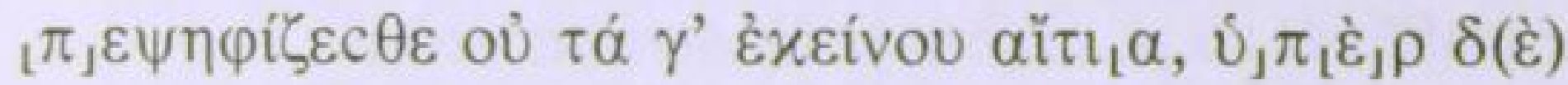

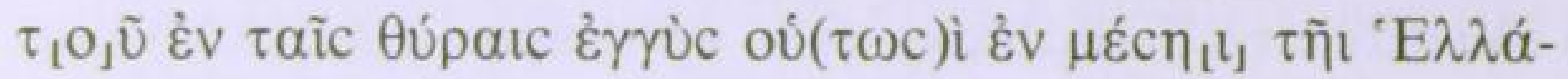

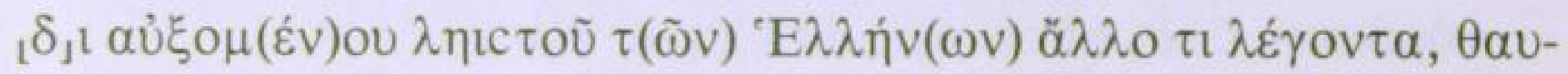

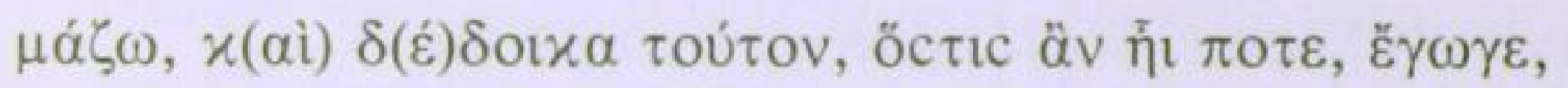

\section{Col. 7}

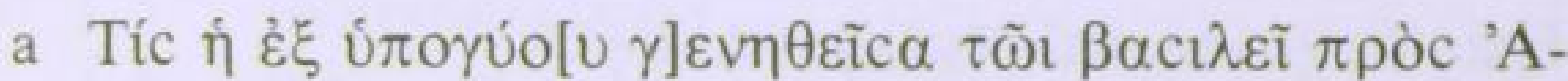

b $\quad$ \#qvaíouc $[\varphi \iota \lambda] \alpha v \theta \rho \omega \pi i \alpha$.

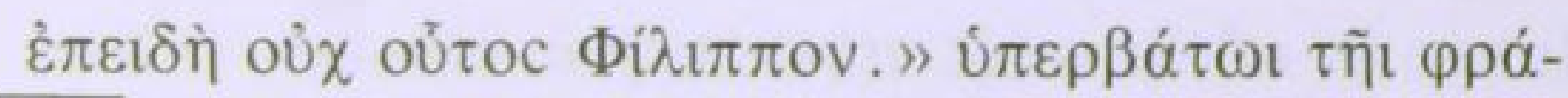

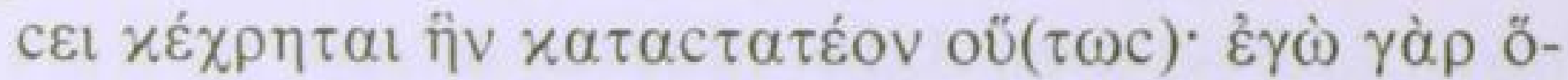

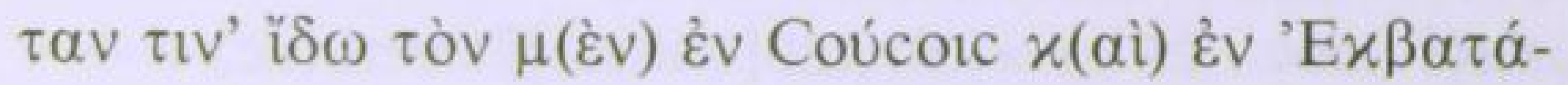

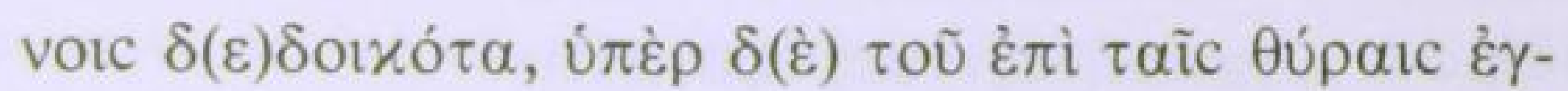




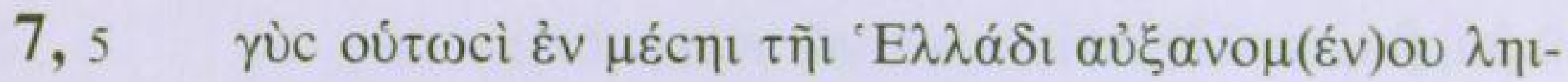

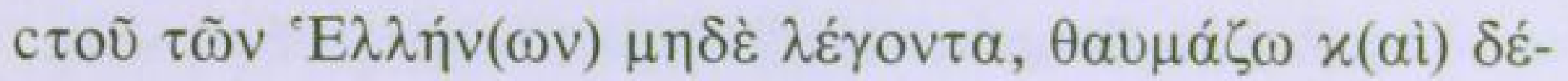

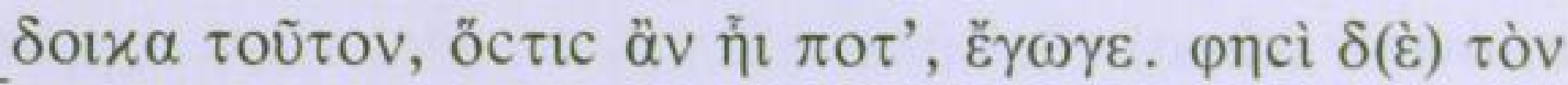
$\beta \alpha c \imath \lambda \varepsilon \dot{\varepsilon} \alpha(\alpha \grave{)}) \pi \rho \tau_{\tau} \varepsilon \rho \circ \nu \mu(\dot{\varepsilon} v) \pi 0 \tau^{\prime} \dot{\varepsilon} \pi \alpha v o \rho \theta \tilde{\omega} c \alpha \iota \tau \dot{\alpha}$

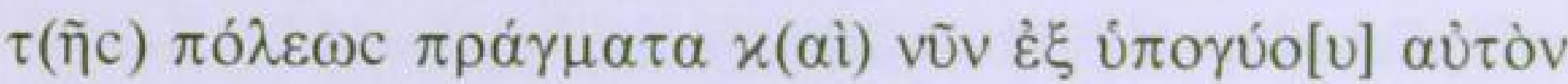

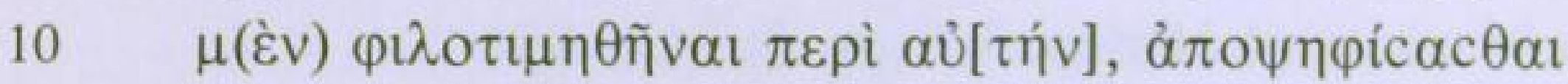

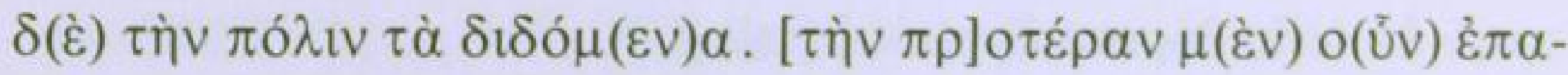

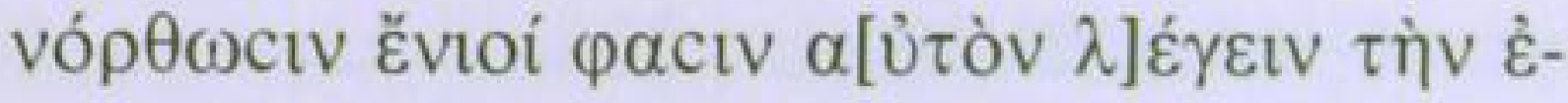

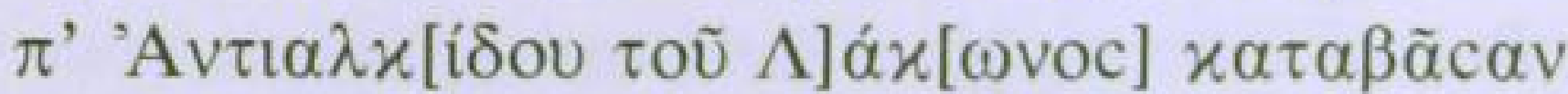

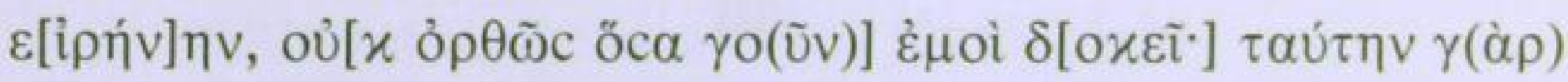

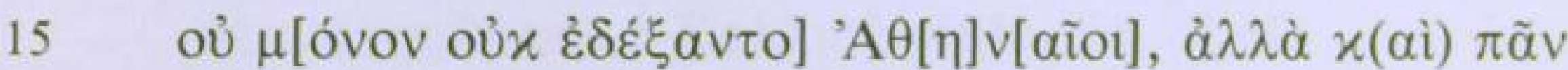

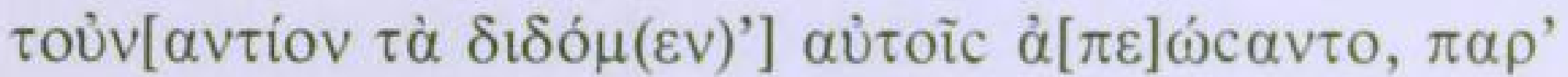

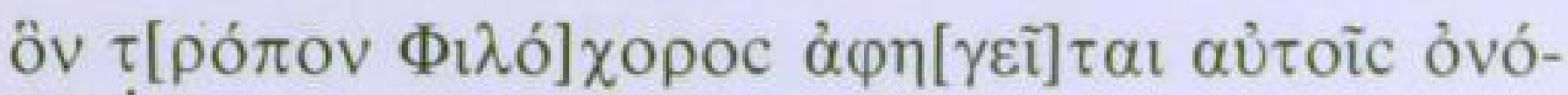

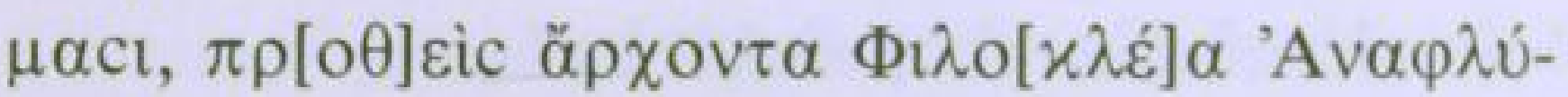

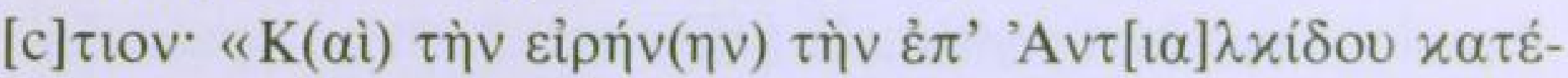

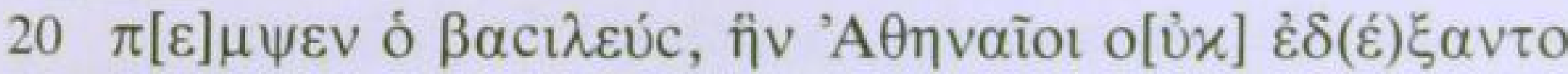

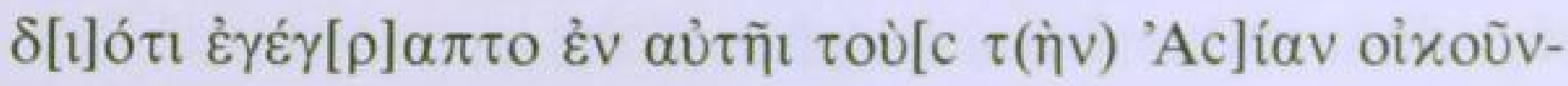

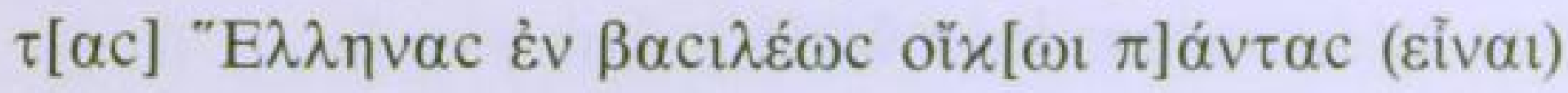

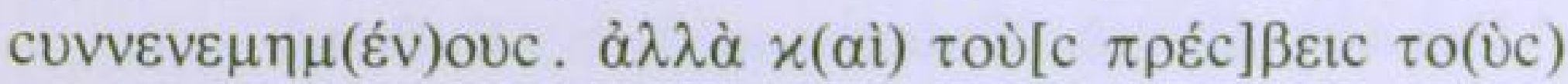

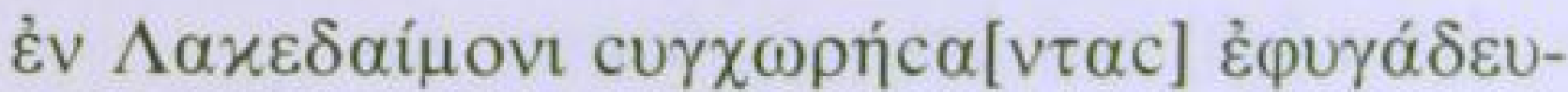

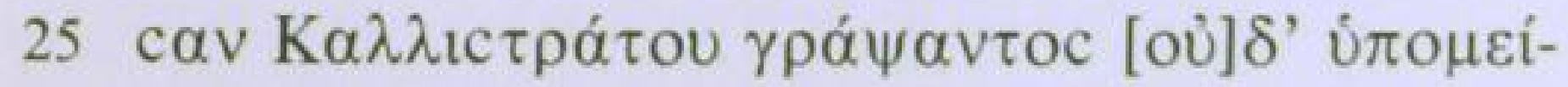

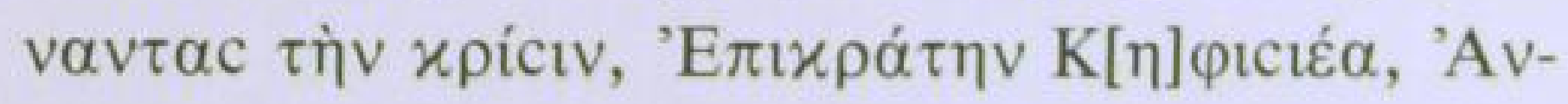




\section{ФІ АІППIК $\Omega \mathrm{N} \bar{\Theta}-\overline{\mathrm{IB}}$}

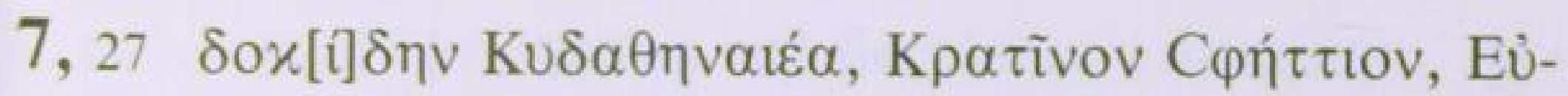

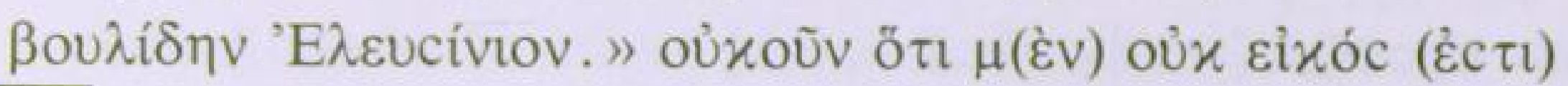

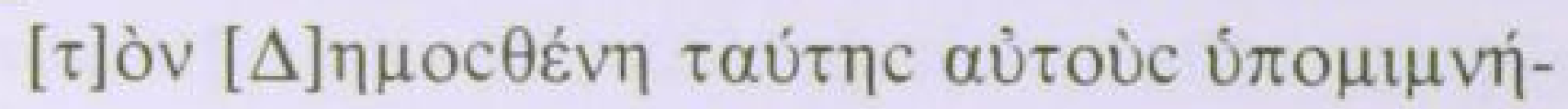

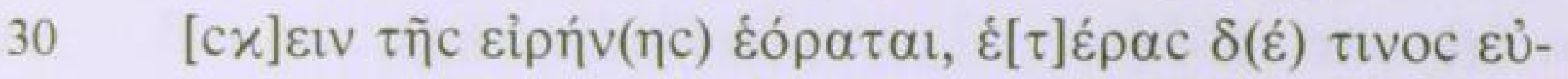

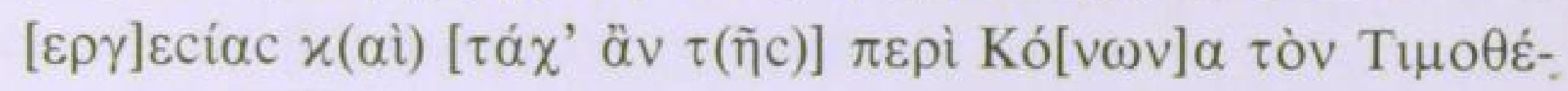

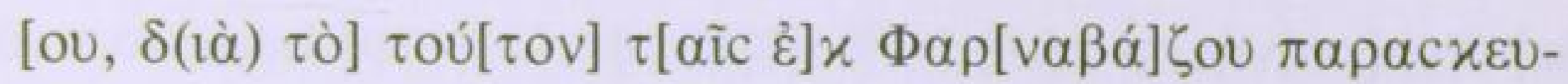

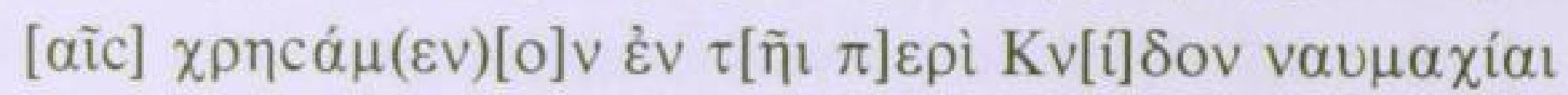

Aug. 394

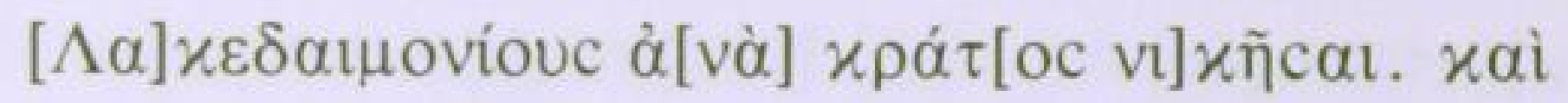

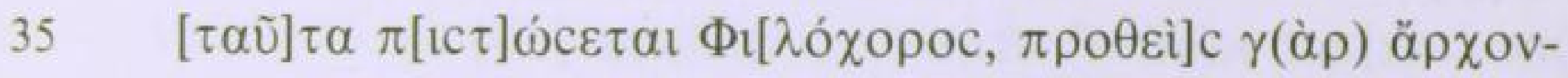

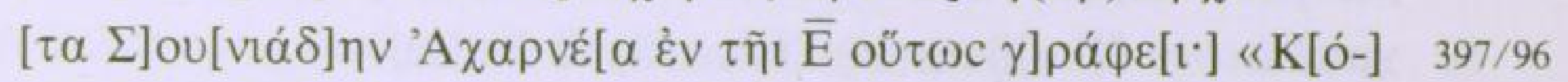

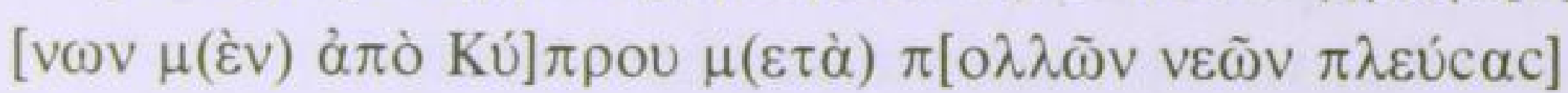

[

$\left[\begin{array}{llll} & 8 & ]\end{array}\right]$

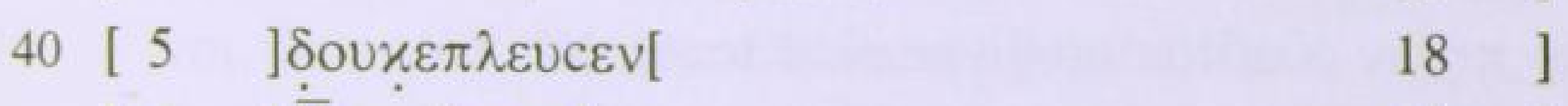

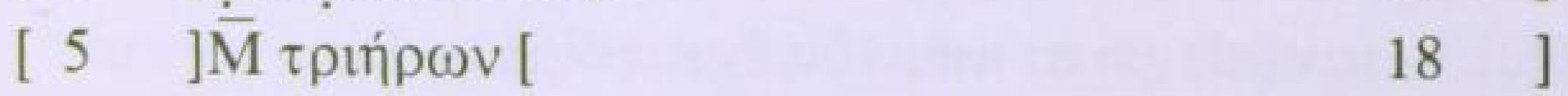




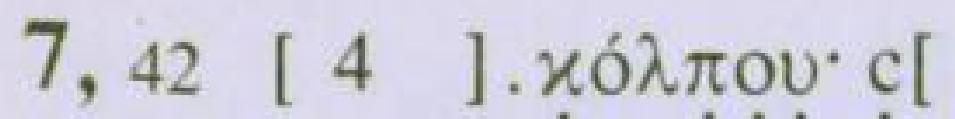

[ 4 ]

[ 3 ] $\alpha \mathrm{c} \tau \grave{\eta} v \pi \alpha \rho \alpha \lambda[\quad 10 \quad$ ]. . [

$\begin{array}{lll} & 18 & ] \\ 7 & & ] \\ 7 & & ]\end{array}$

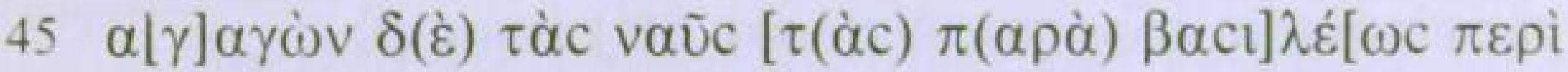

$\Lambda] \omega \dot{\rho} \rho \mu[\alpha]$

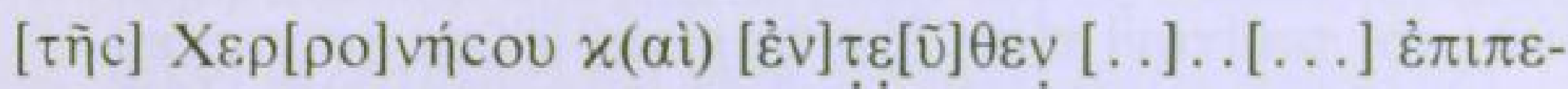

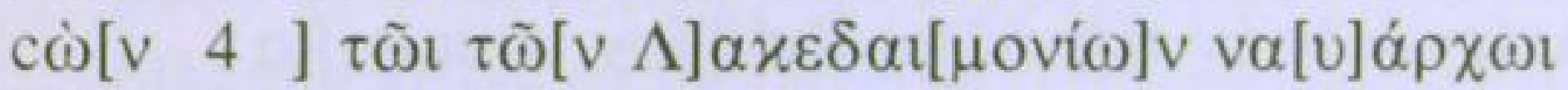

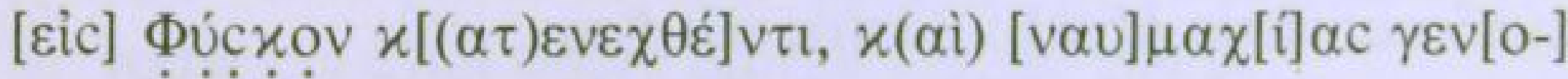

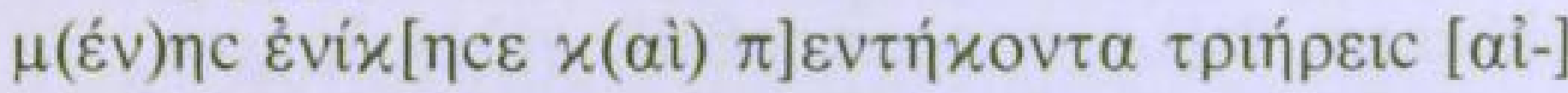

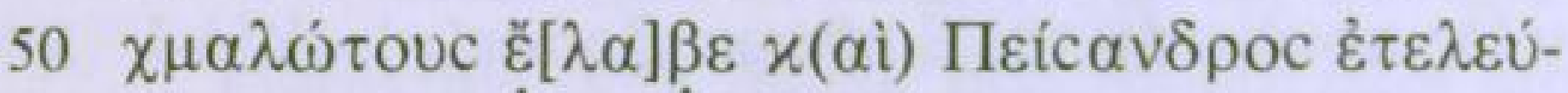

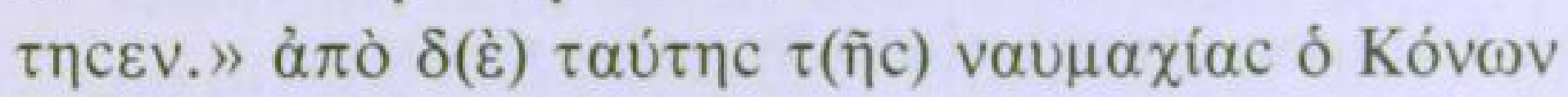
$\chi(\alpha i) \tau \grave{\alpha}[\mu \alpha x \rho \alpha \grave{\alpha} \tau] \varepsilon i ́ \chi \eta \tau o i ̃ c ~ ' A \theta \eta v \alpha i ́ o[[c] ~ \alpha ̀ v \varepsilon ́-$

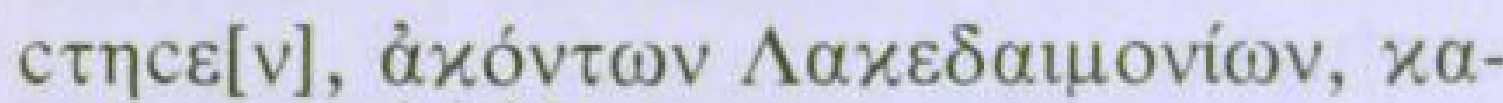

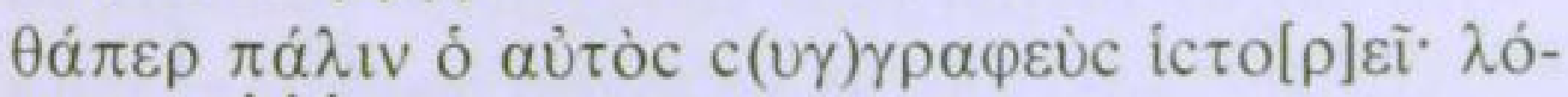

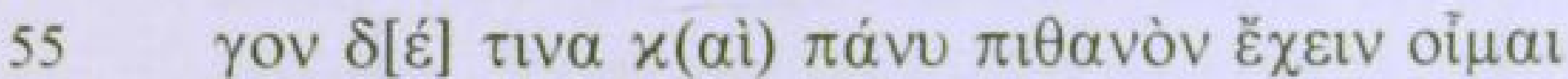

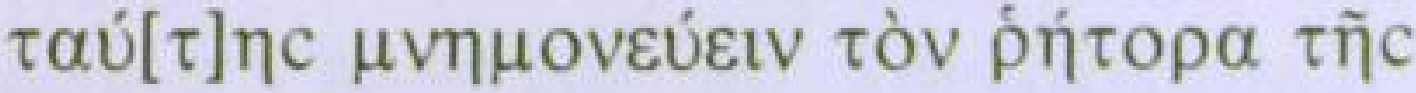




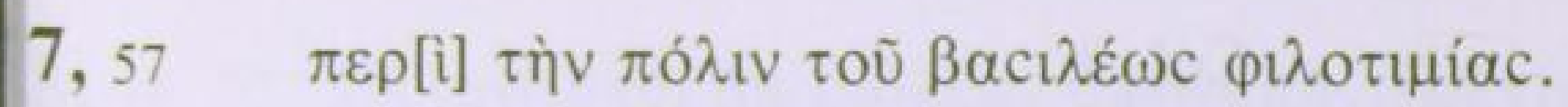

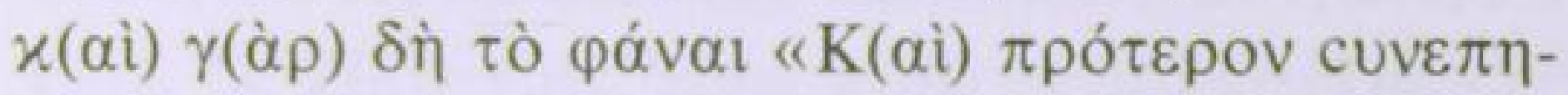

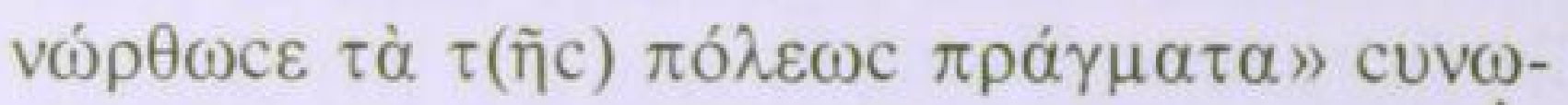

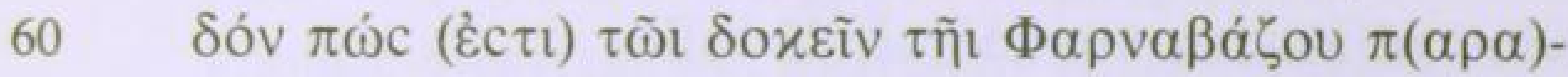

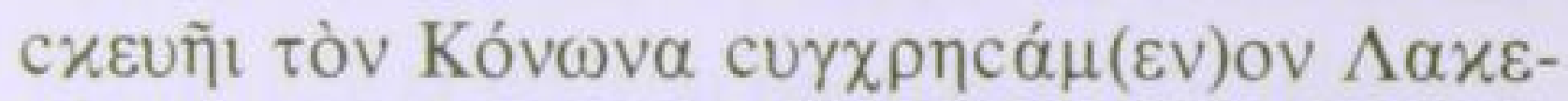

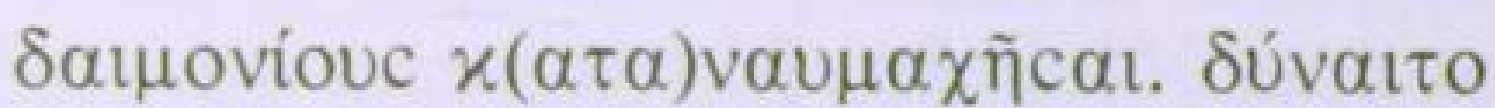

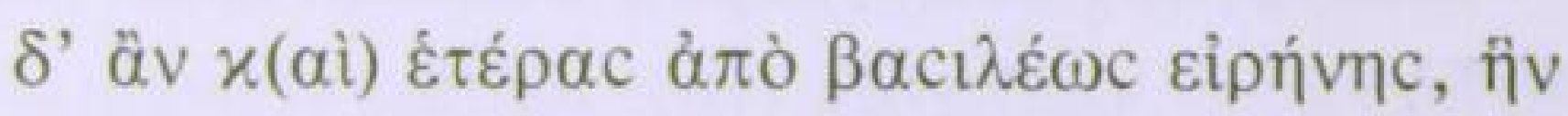

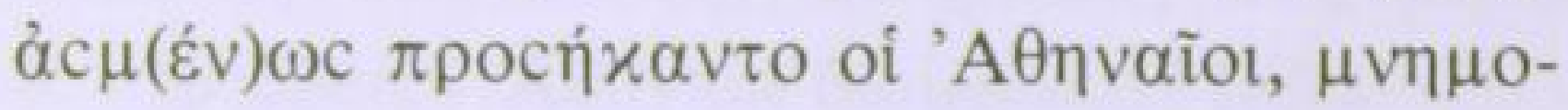

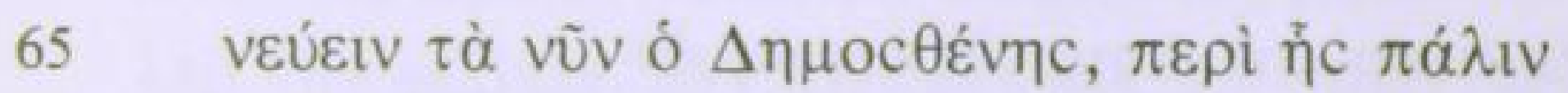

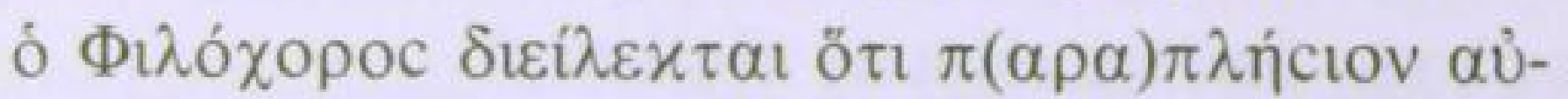

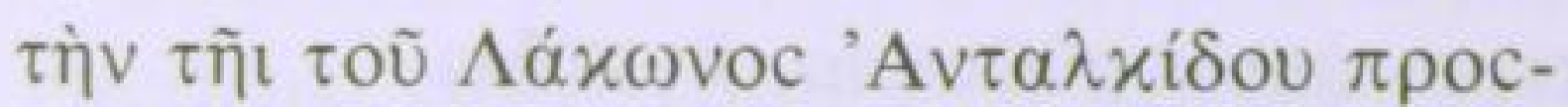

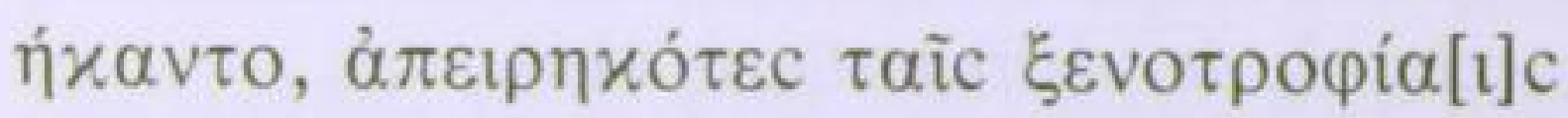

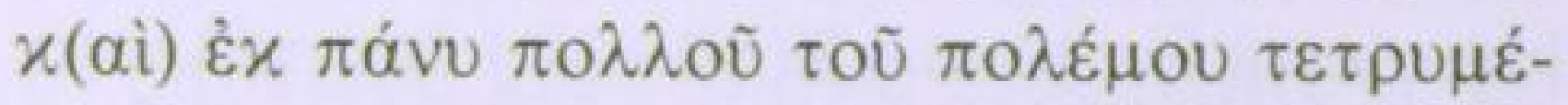

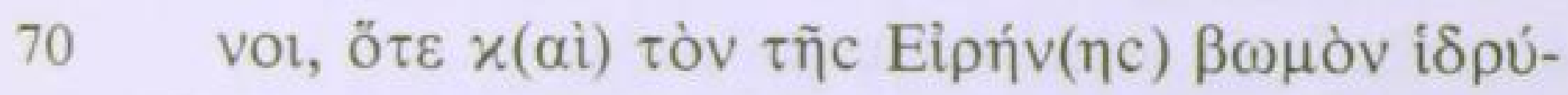

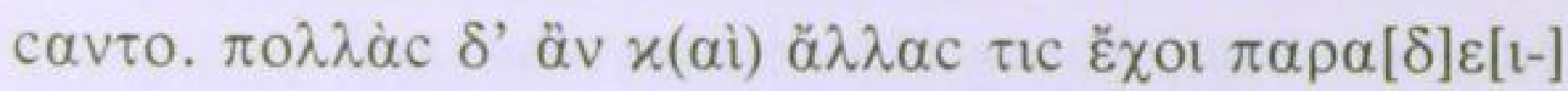

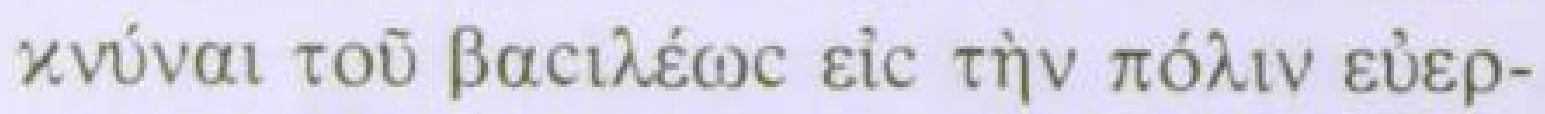

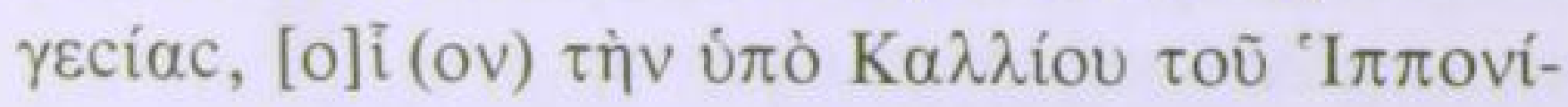

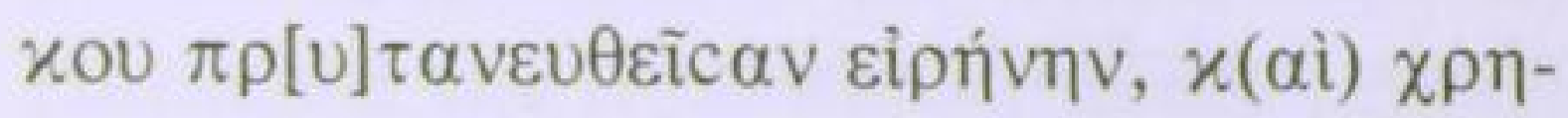

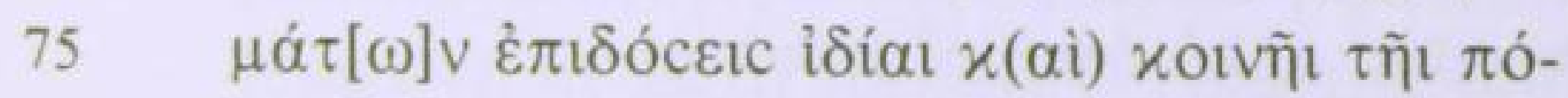


Col. 8

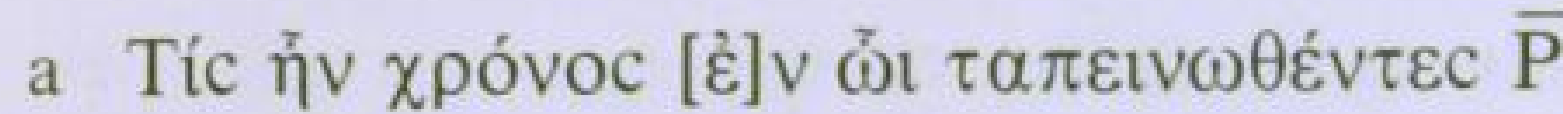

b $x \alpha \grave{i} \bar{\Lambda} \mu[o ́ v]$ ov $\tau \alpha \dot{\alpha}[\lambda \alpha v] \tau \alpha \pi \rho o c o ́ \delta[o] v \dot{\varepsilon} \lambda \alpha \dot{\alpha} \mu \beta \alpha$ -

c vov.

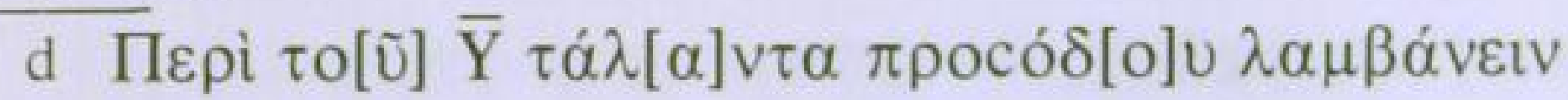

e toùc ['A] $\theta$ mvaíouc.

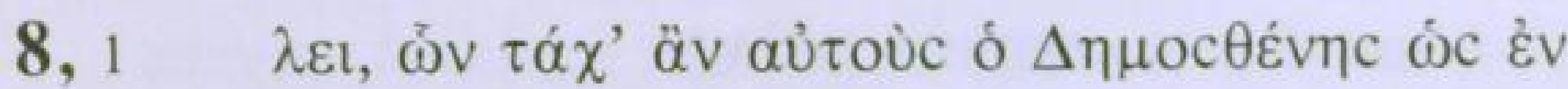

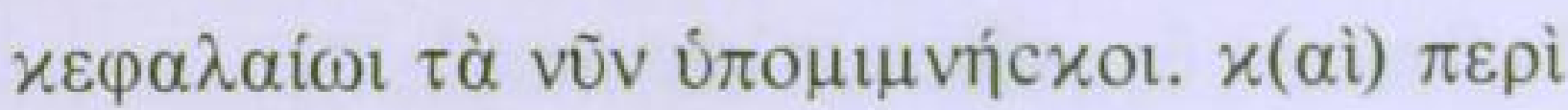

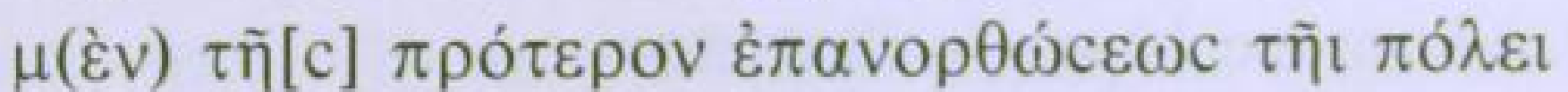

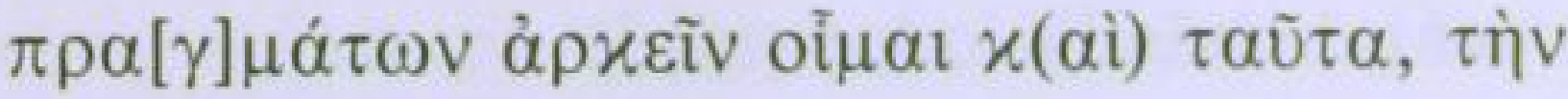

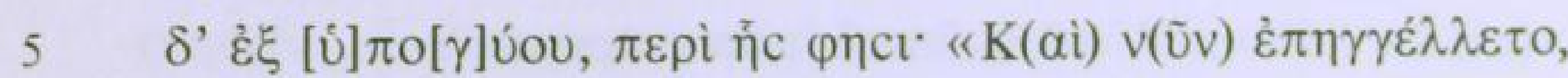

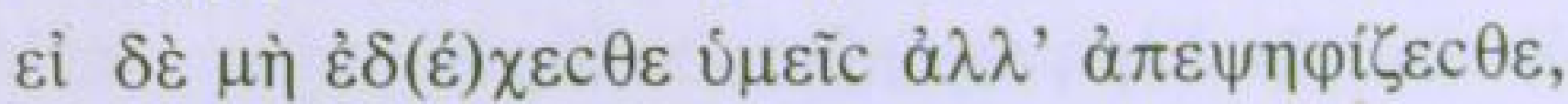

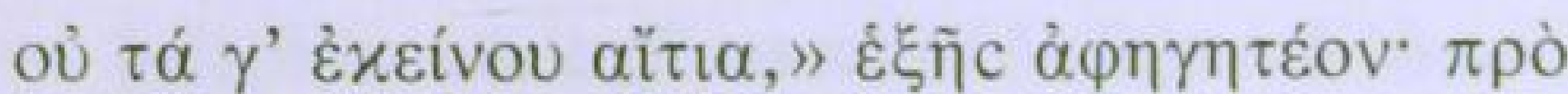

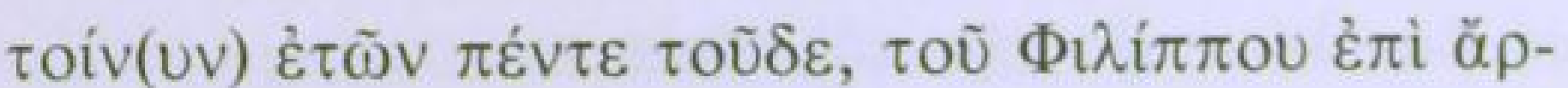

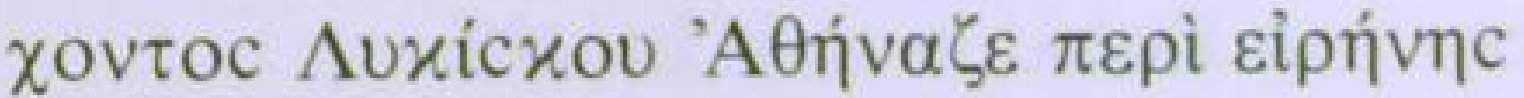

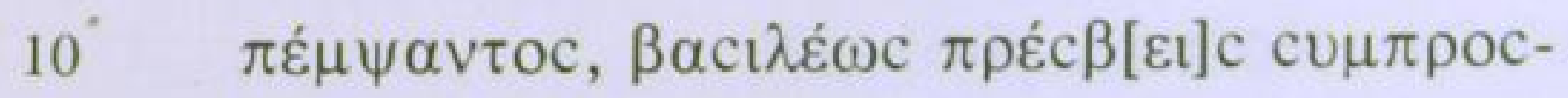

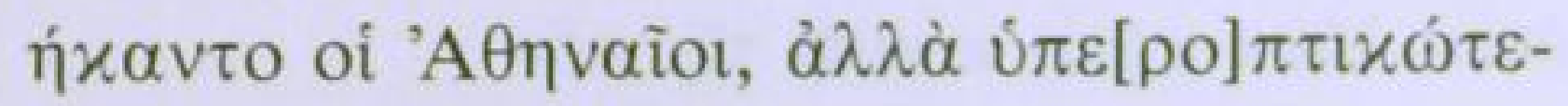

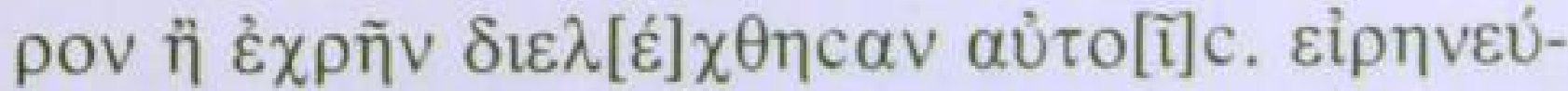

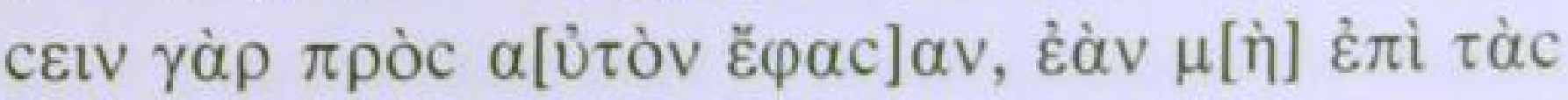




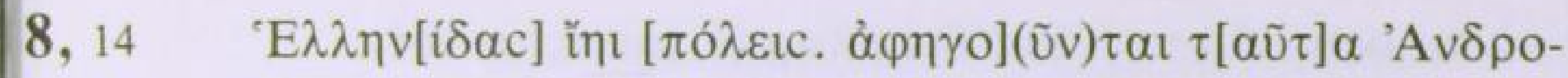

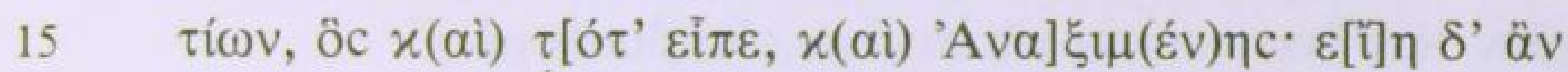

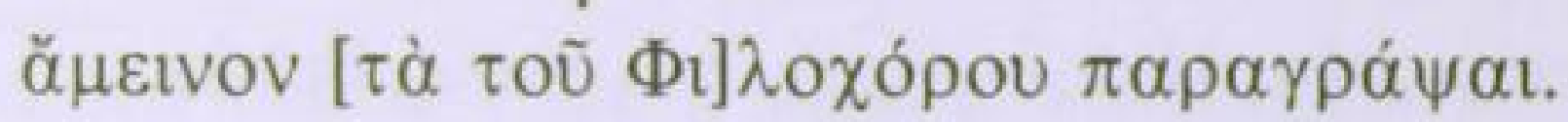

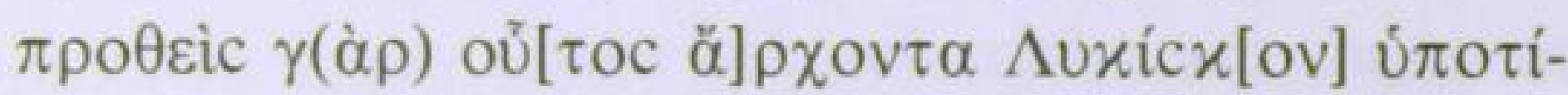

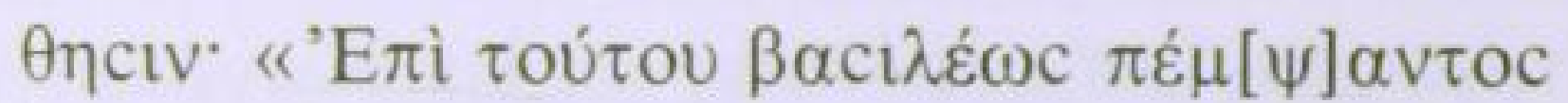

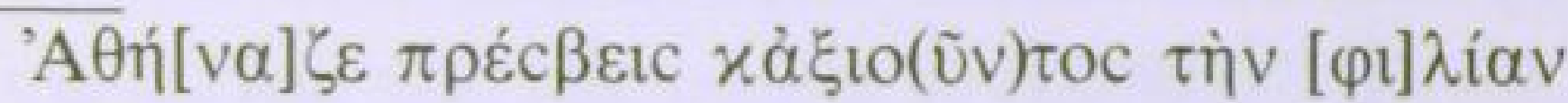

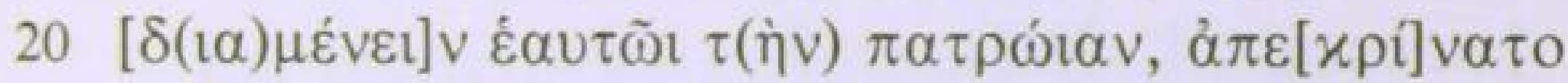

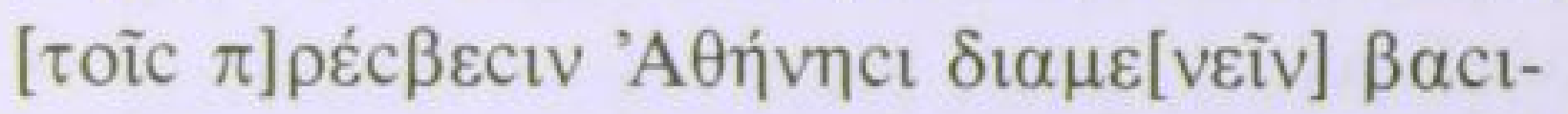

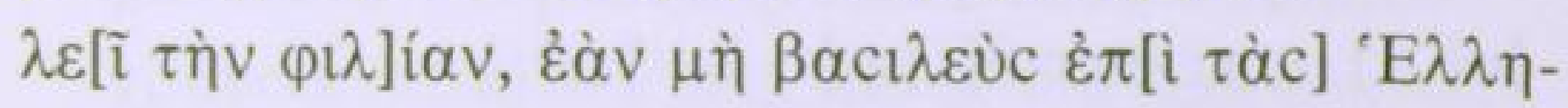

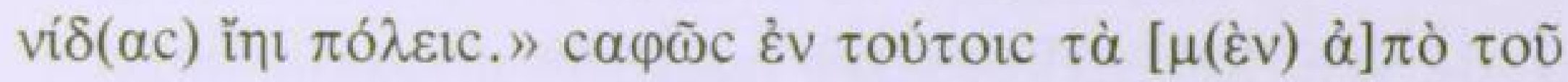

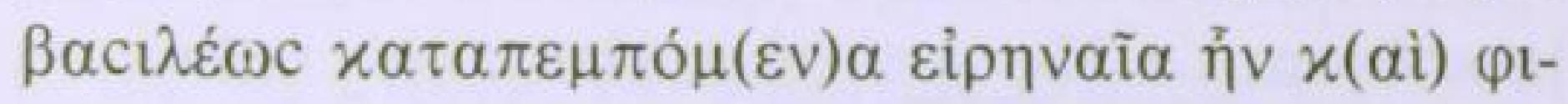

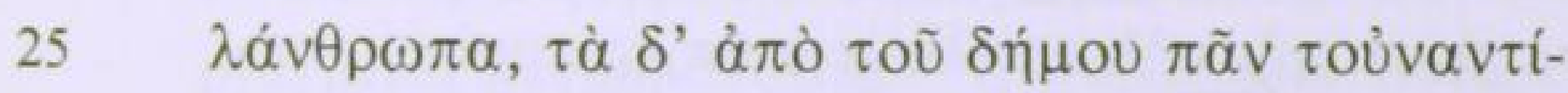

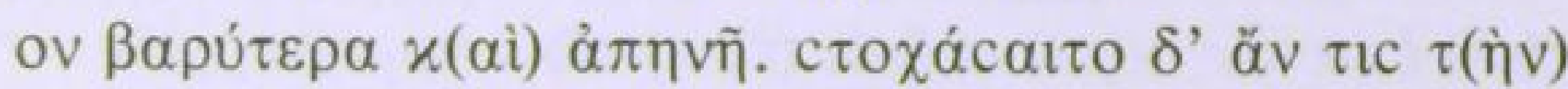

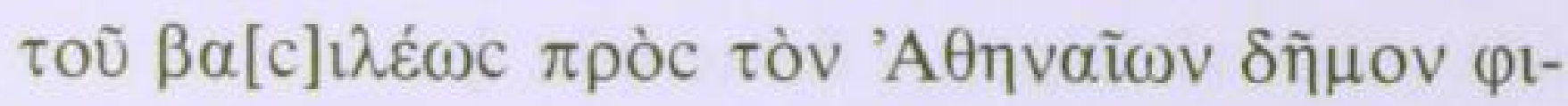

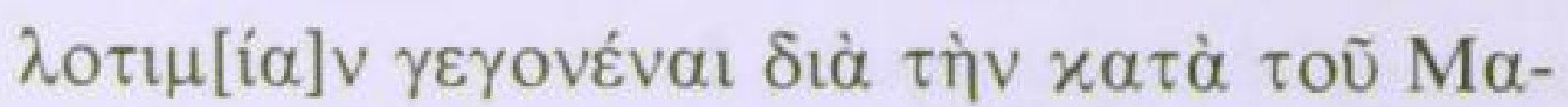

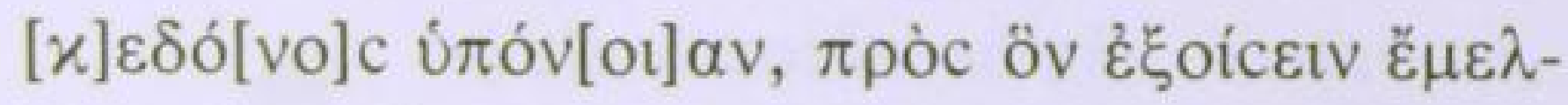

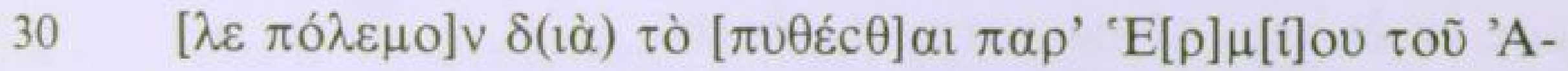

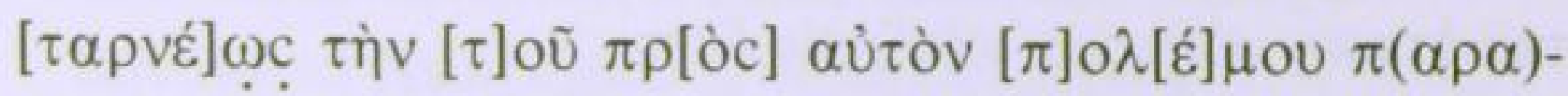

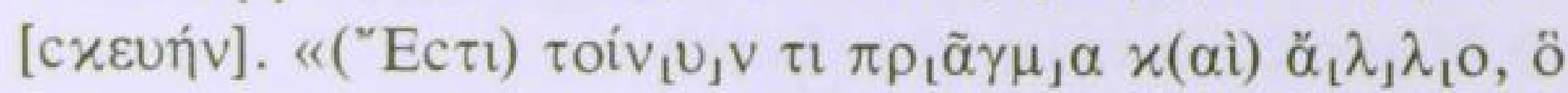

$\lambda_{\mathrm{j}} \mathrm{v \mu \alpha i-}$

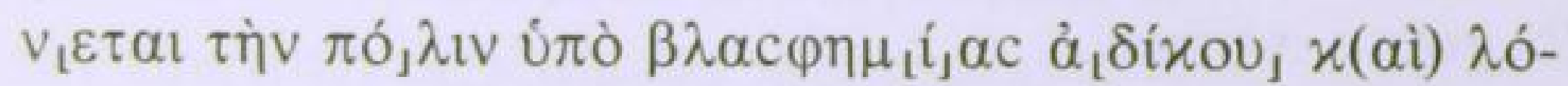




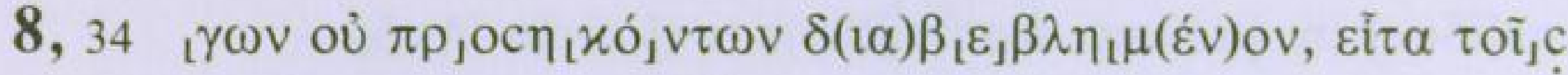

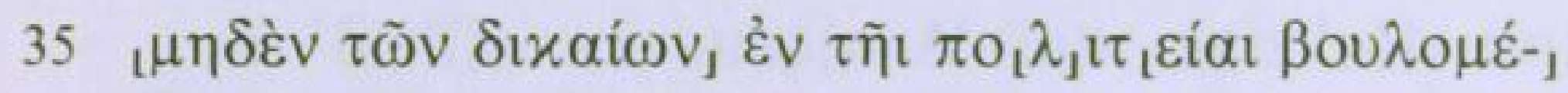

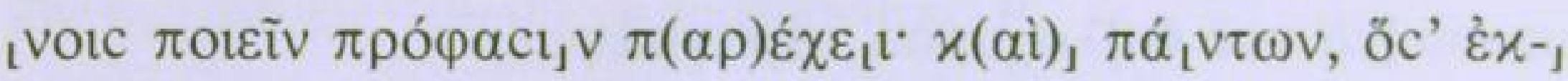

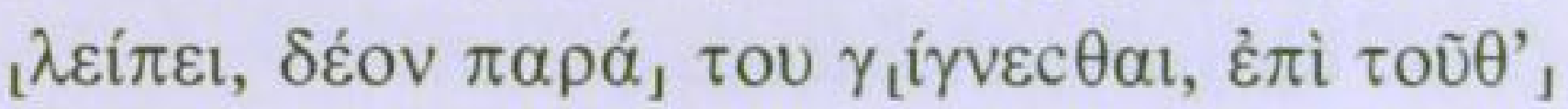

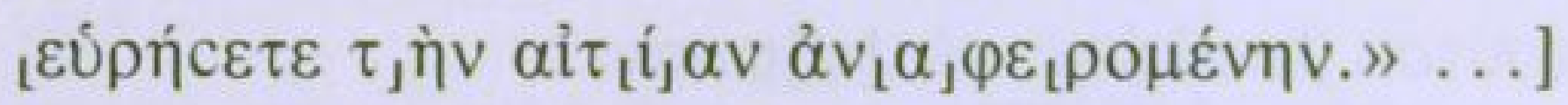

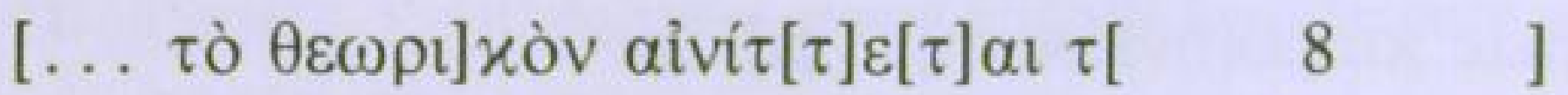

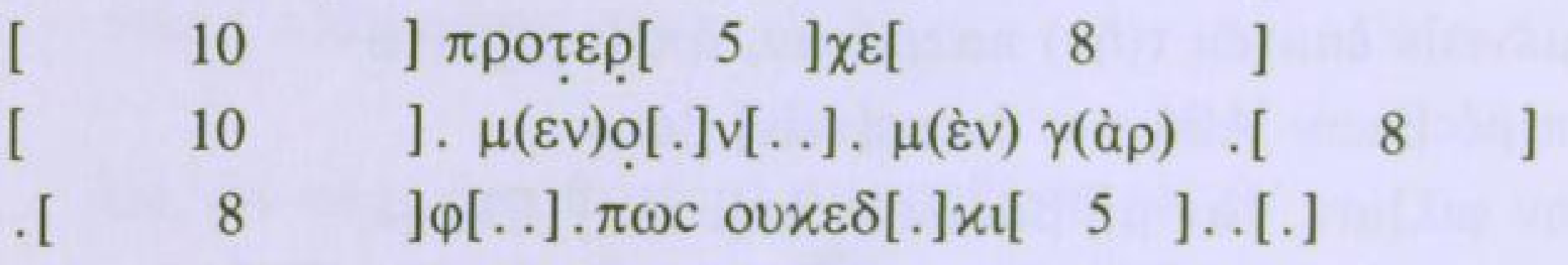

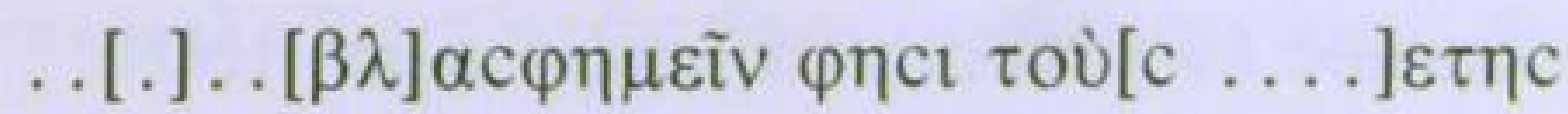

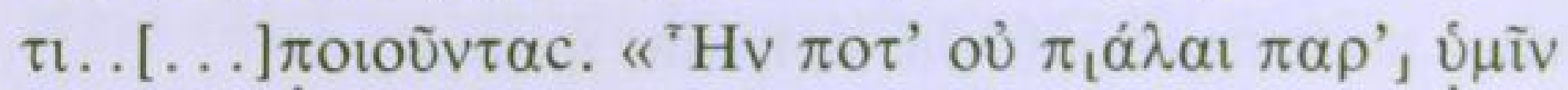

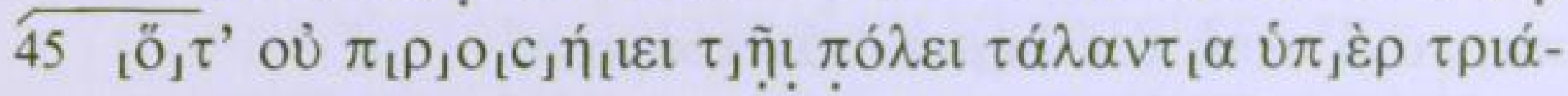

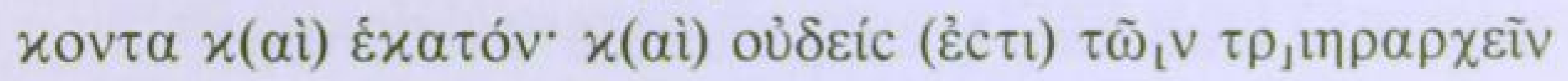

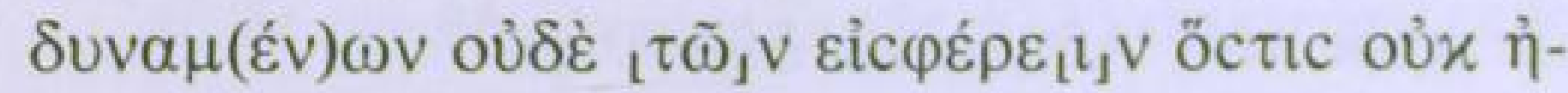

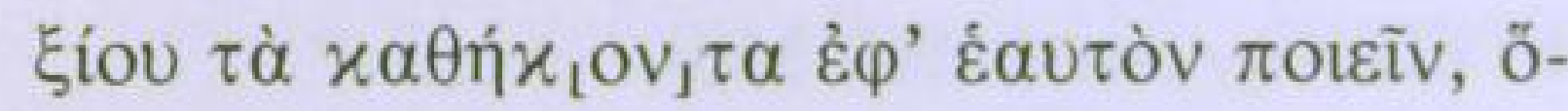

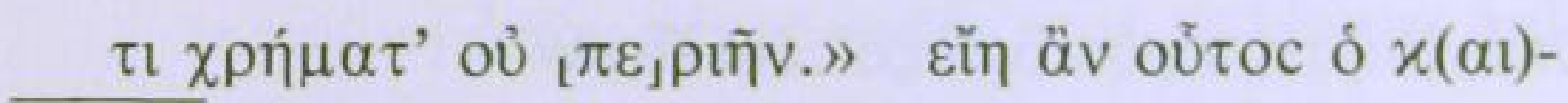

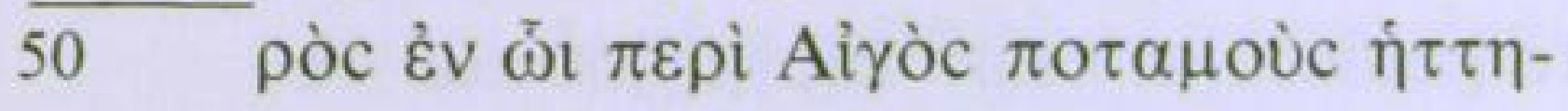

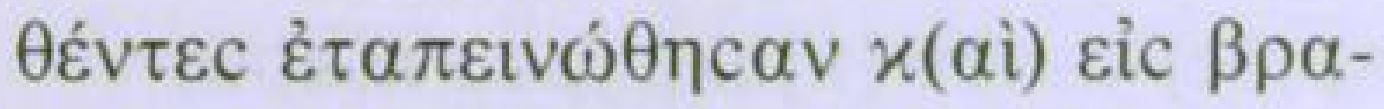

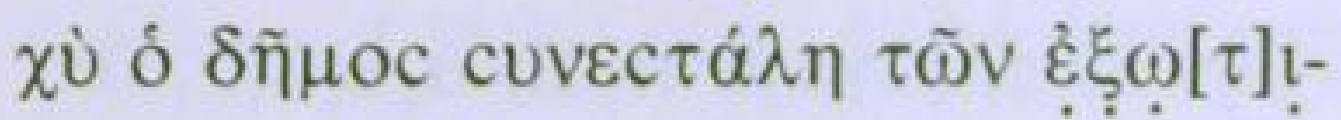

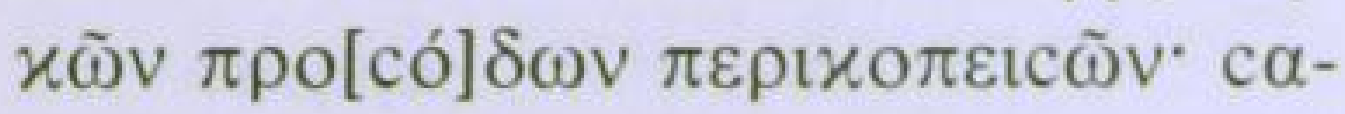

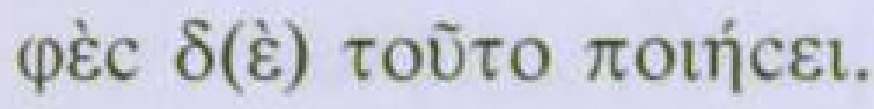


Spatium fere decem versuum vacat.

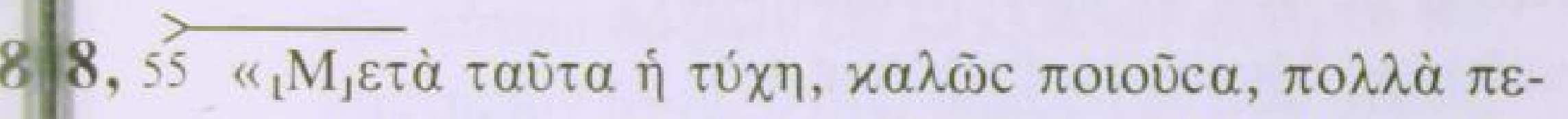

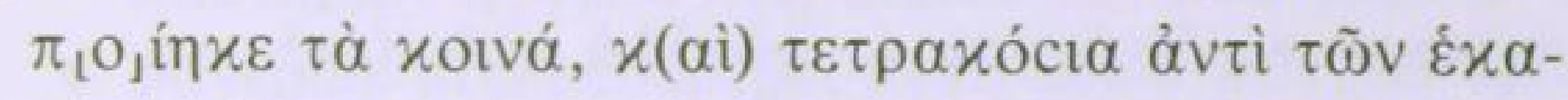

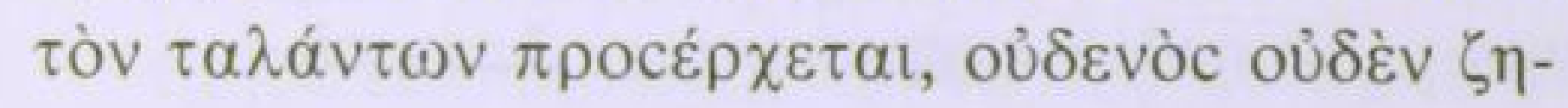

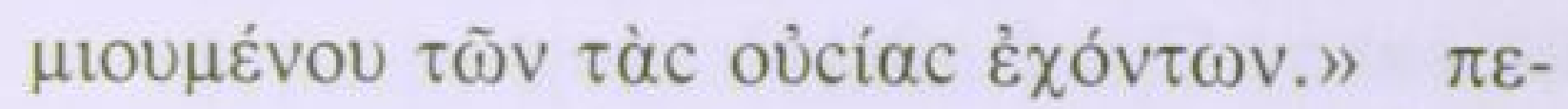

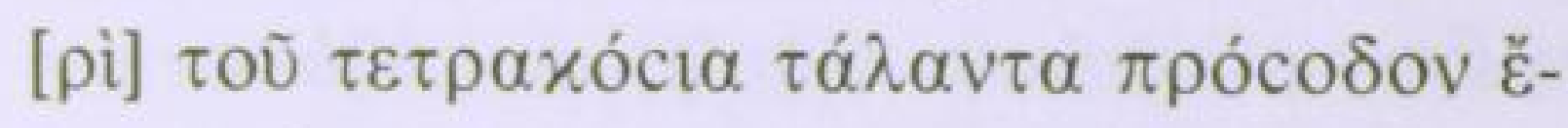

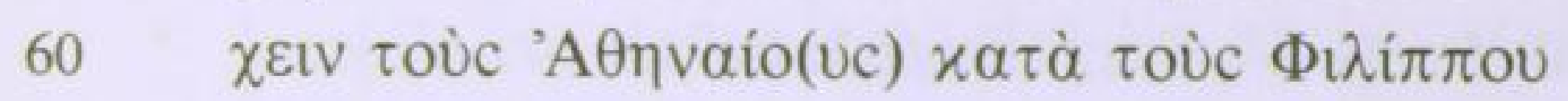

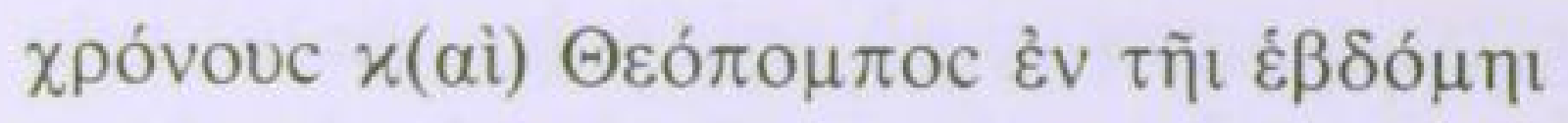

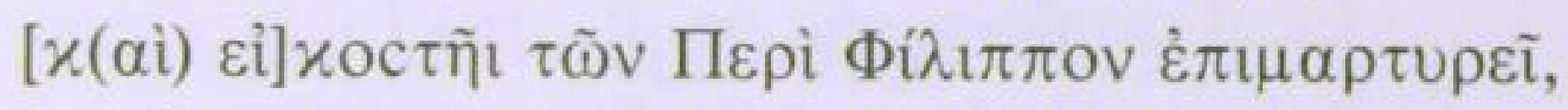

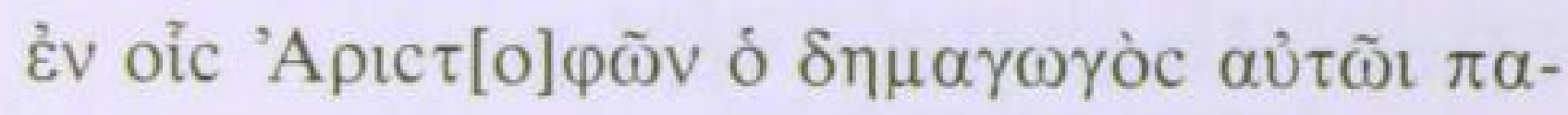

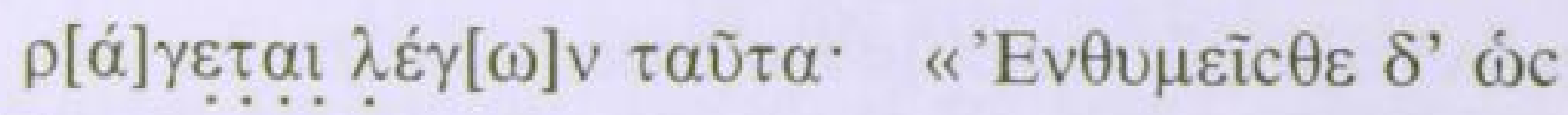

\section{Col. 9}

a

b

"O $\tau$ в B 'A

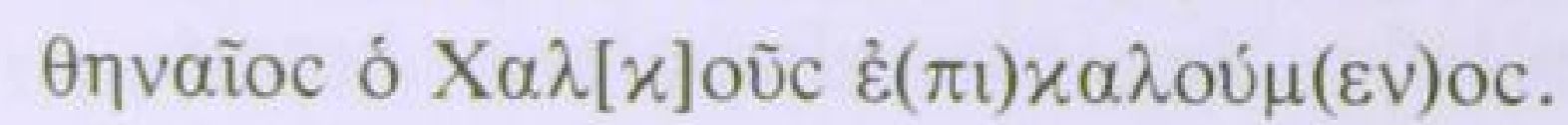

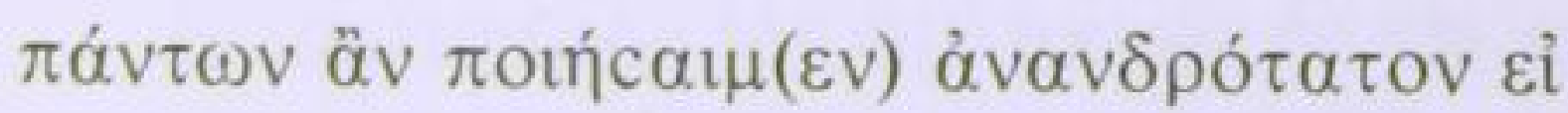

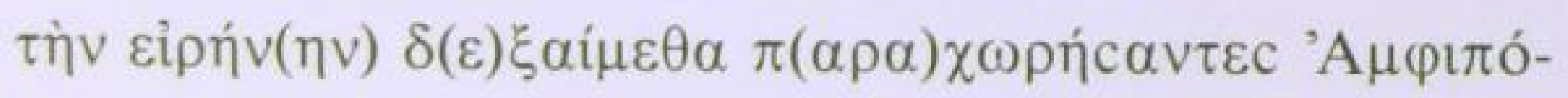

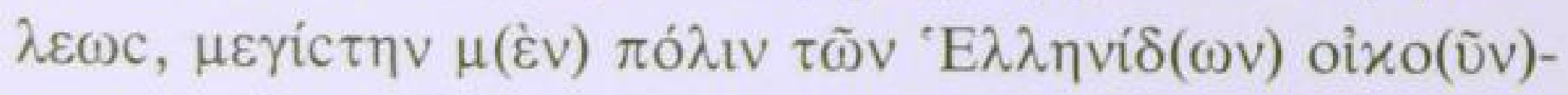

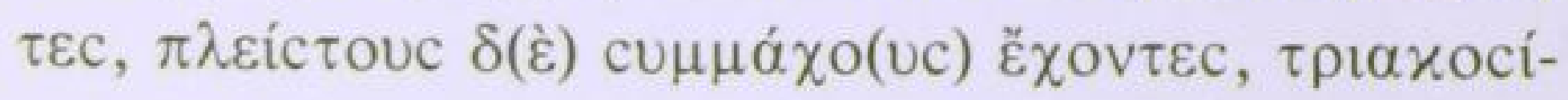

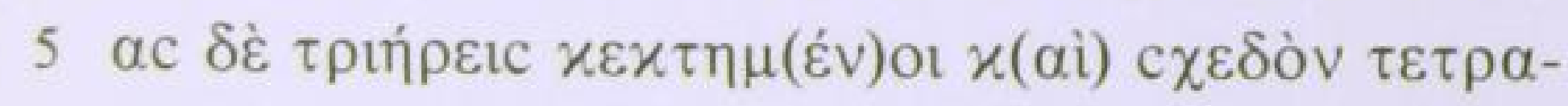




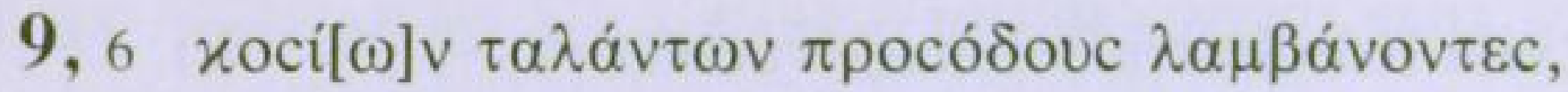

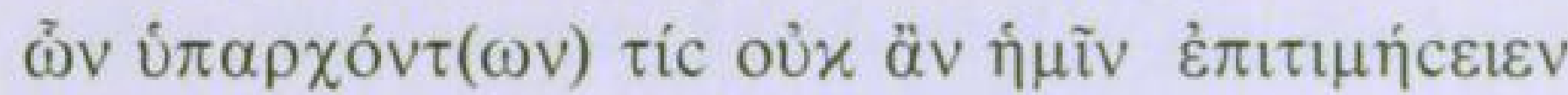

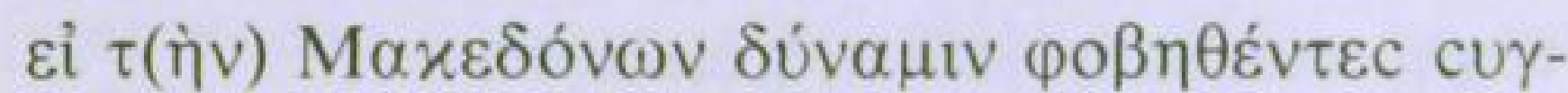

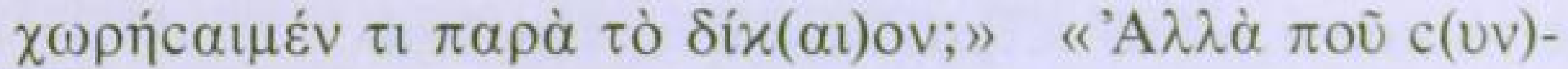

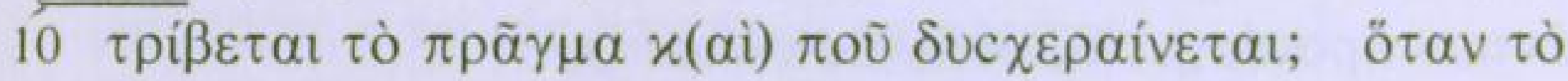

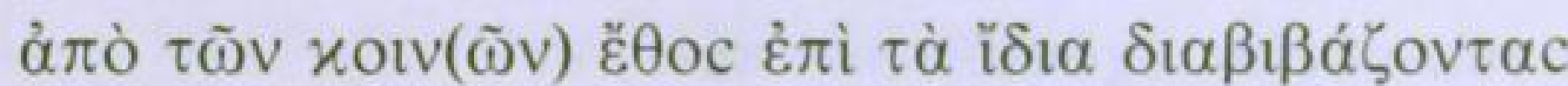

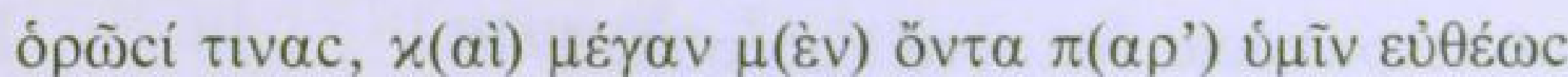

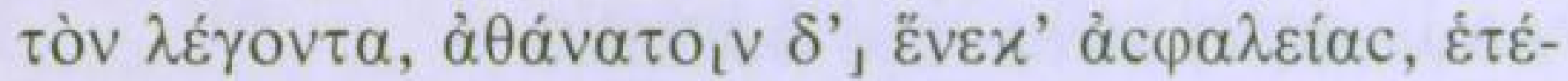

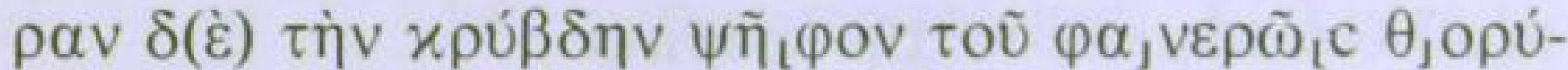

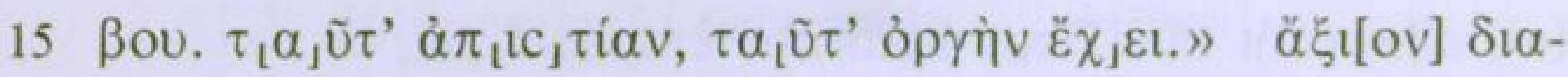

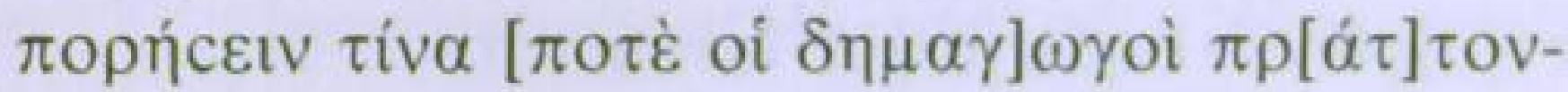

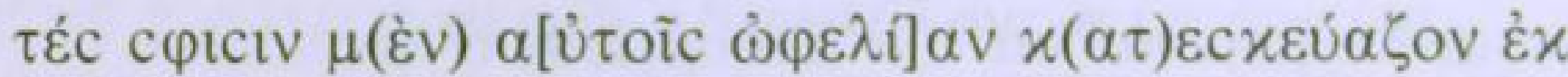

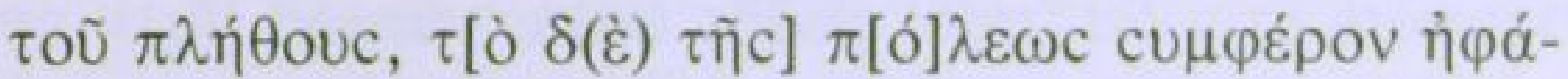

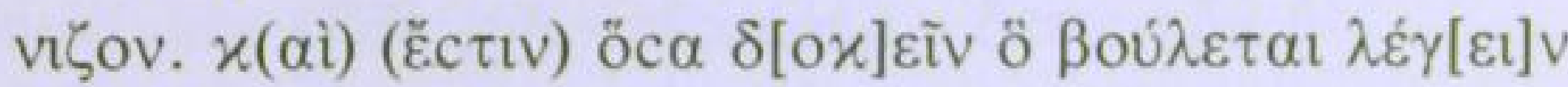

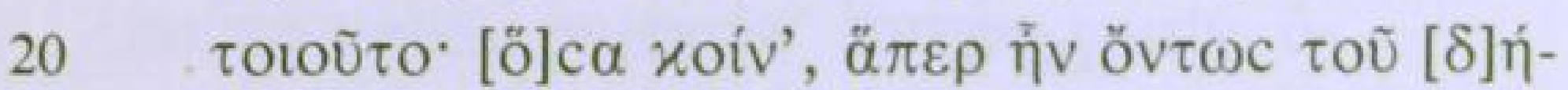

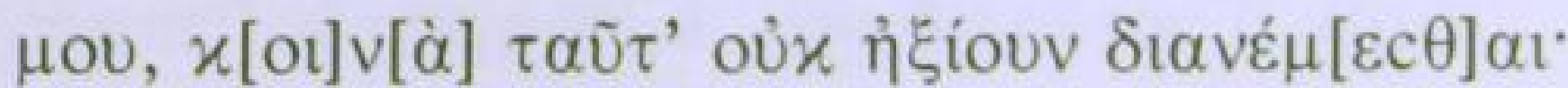
$\dot{\alpha} \lambda\left[\lambda \lambda^{\prime} \dot{\varepsilon} \pi \varepsilon\right] \mu[\eta] \chi \alpha \nu \tilde{v} \nu \tau o \quad x(\alpha i) \tau \tilde{\omega} v$ ov่ $\delta เ x(\alpha i) \omega[v i \delta i] \alpha c$

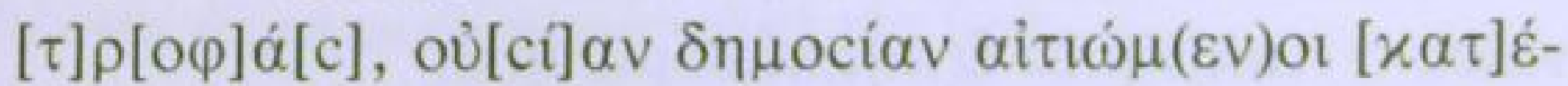

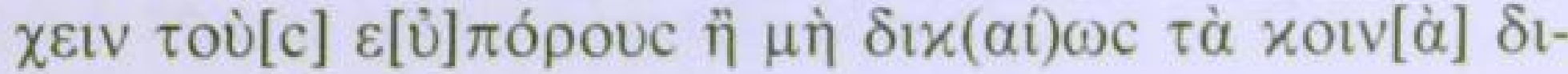




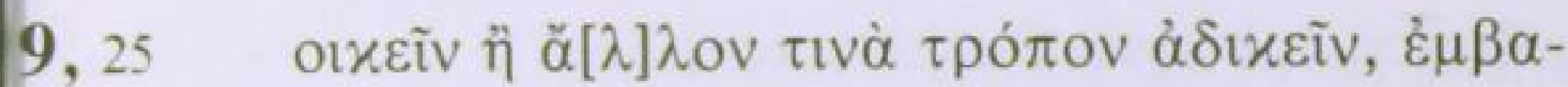

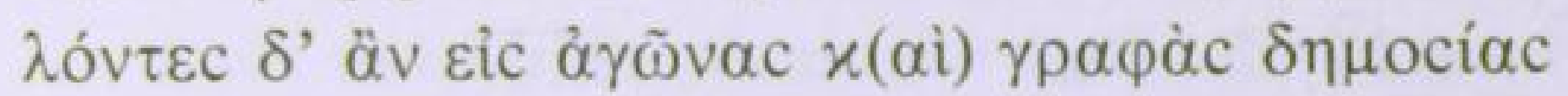

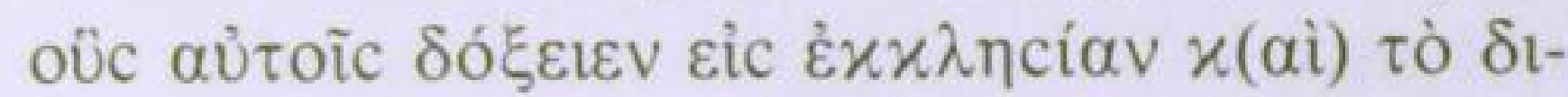

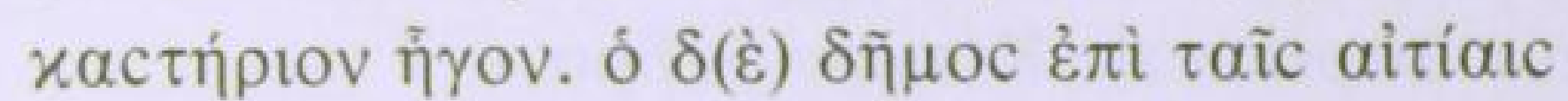

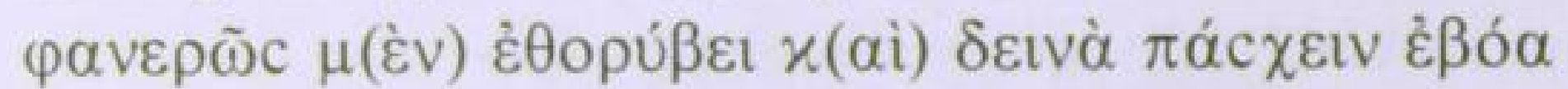
$\tau[o] \cup ̀ c ~ \varepsilon u ̉ \pi o ́ \rho[o(v c)], x \rho u ́ \varphi \alpha \delta(\grave{\varepsilon}) x \alpha \tau \varepsilon \psi \eta \varphi i \zeta ̌ \tau o ~ x(\alpha i)$ $\pi[0] \lambda \lambda \tilde{\omega} \nu \dot{\varepsilon}[\tau i] \mu \alpha \chi \rho[\eta \mu \alpha \dot{\tau} \omega] \nu . \tau \alpha \tilde{\tau} \alpha \delta(\grave{\varepsilon}) \dot{\varepsilon} \gamma \dot{-}$

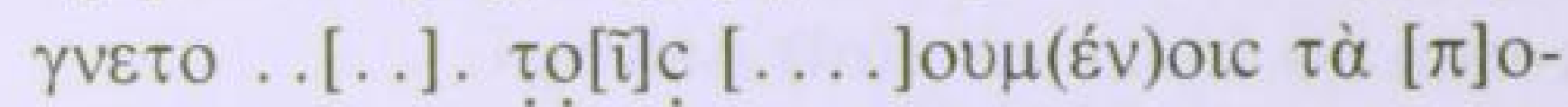

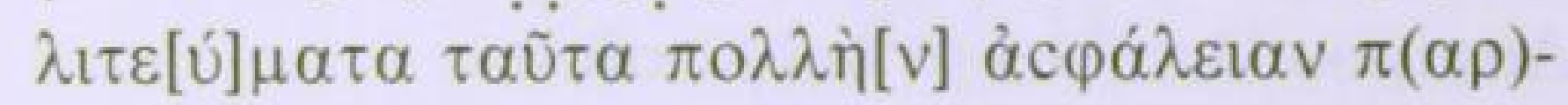

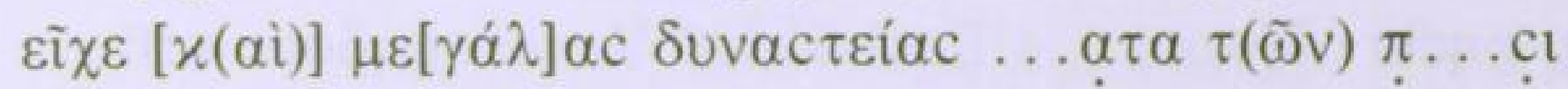

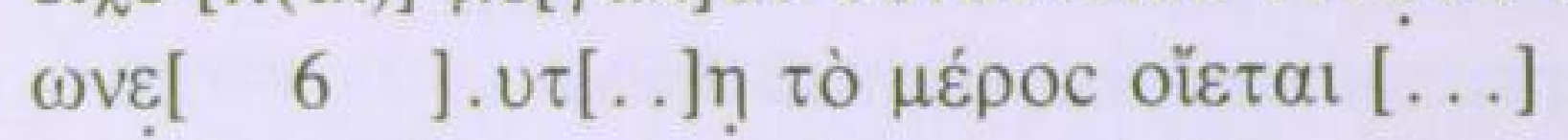

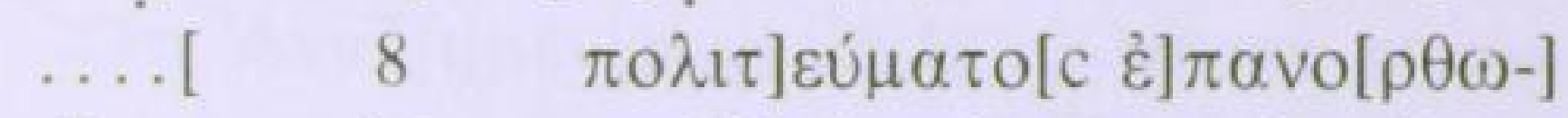
[ [ $15 \quad] \delta^{\prime} \omega \mathrm{c} \varepsilon \bar{\chi} \chi \varepsilon x \alpha \tau \alpha[\ldots]$

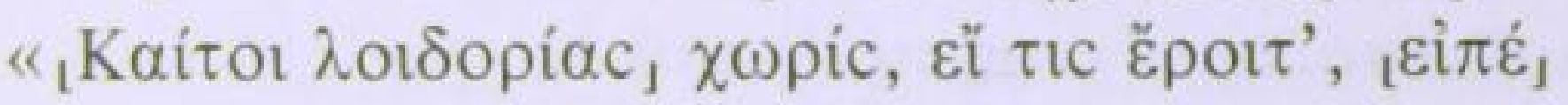

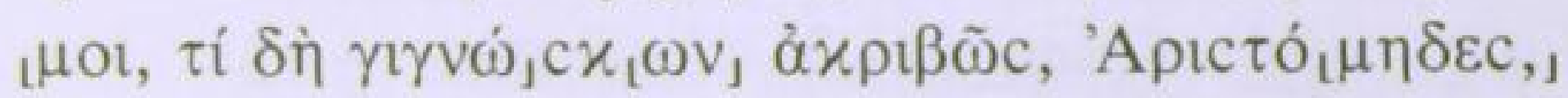

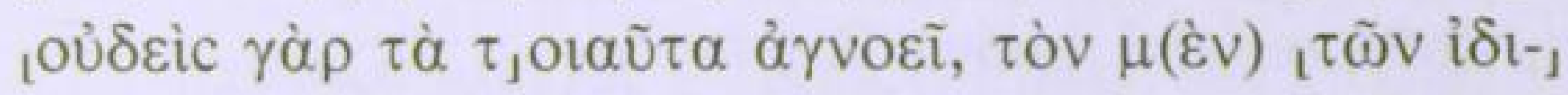

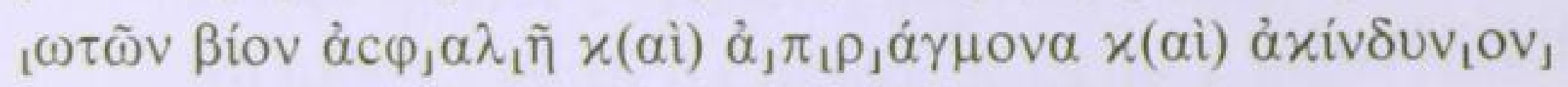

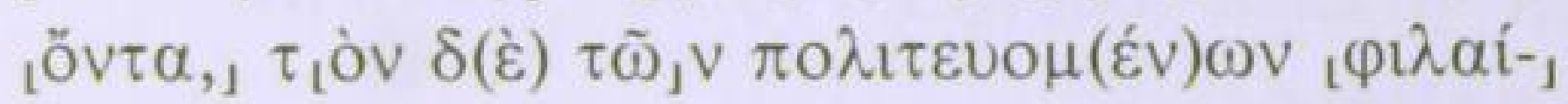

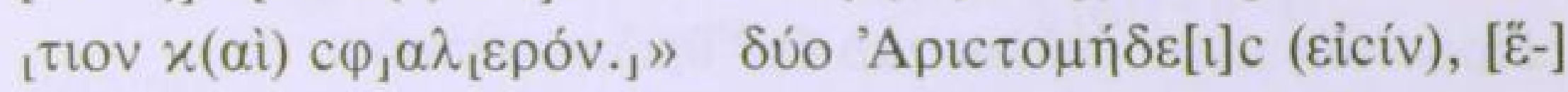

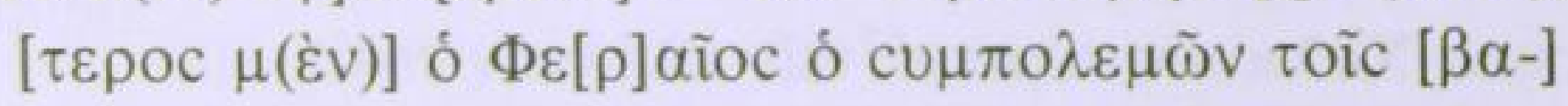

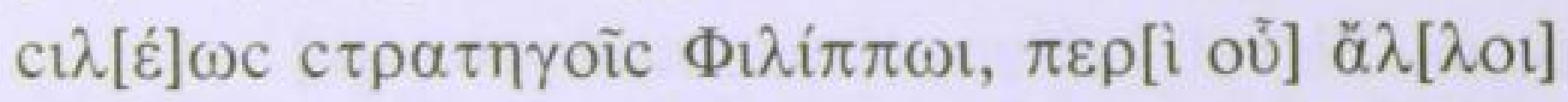

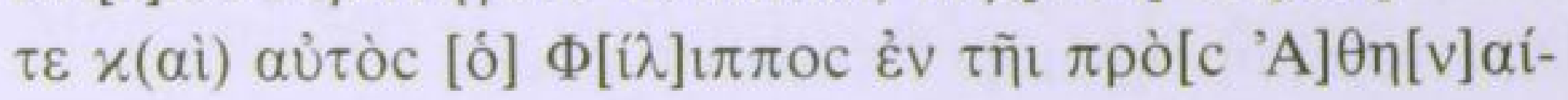




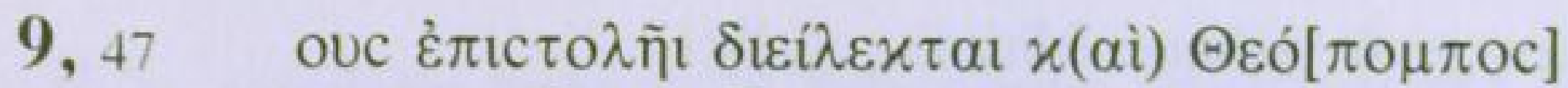

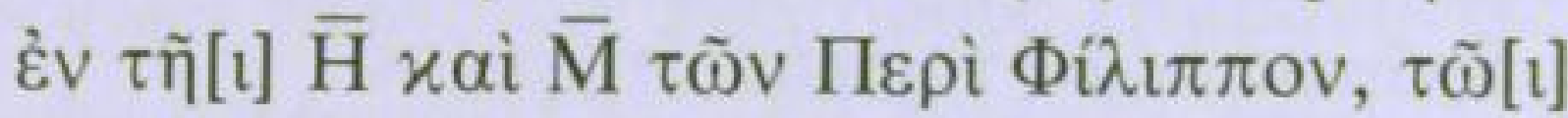

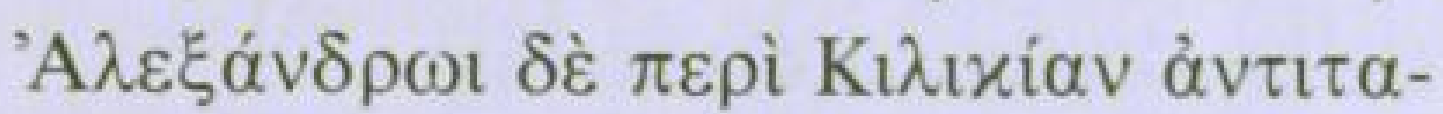

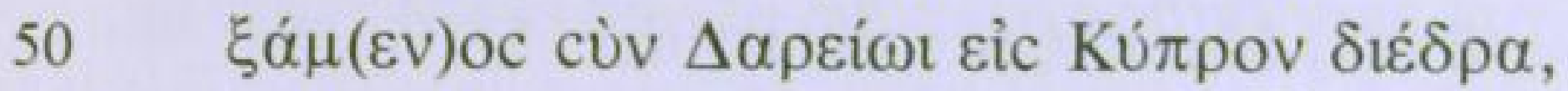

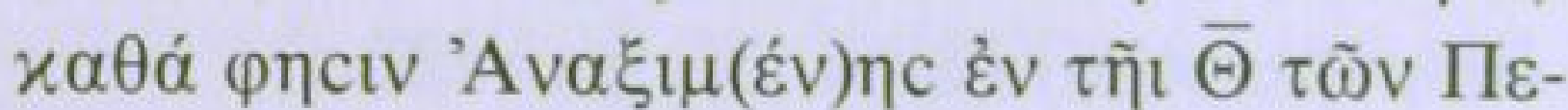

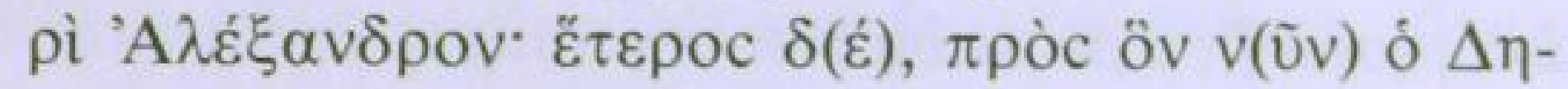

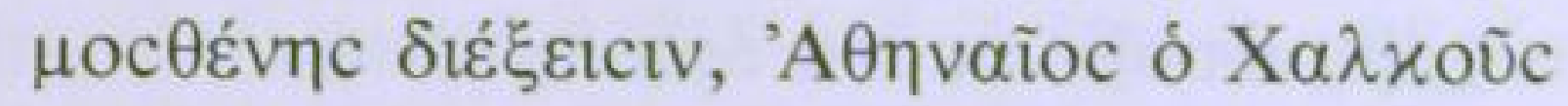

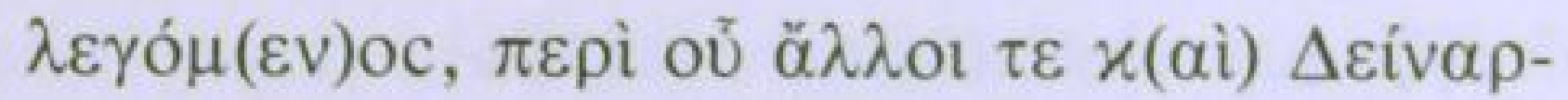

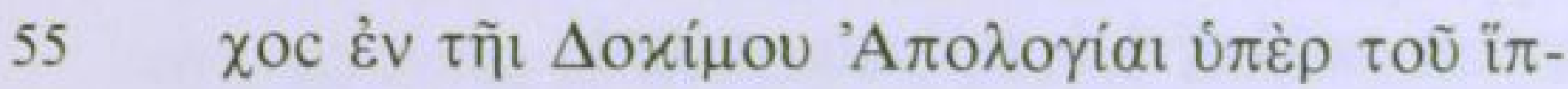

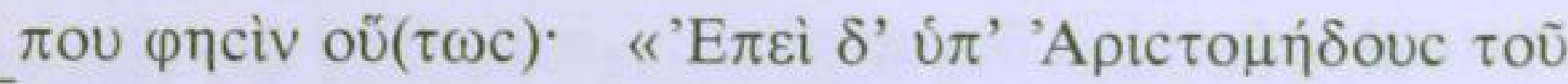

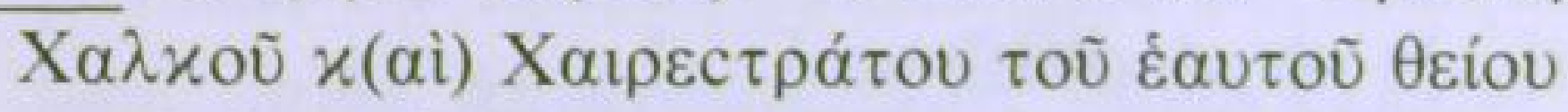

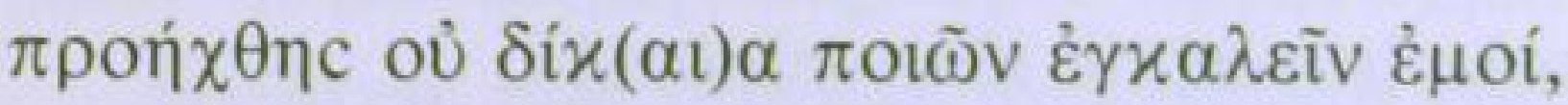

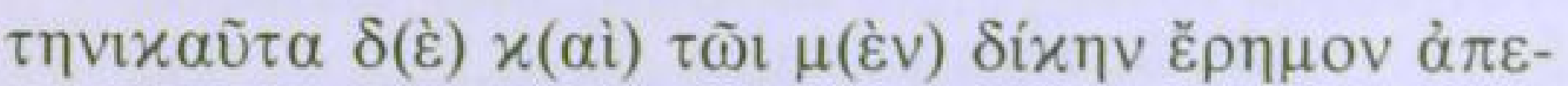

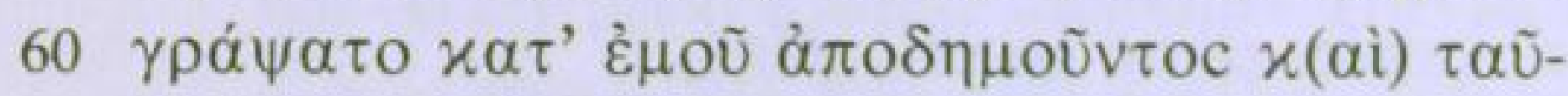

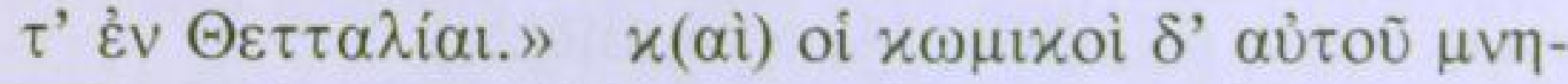

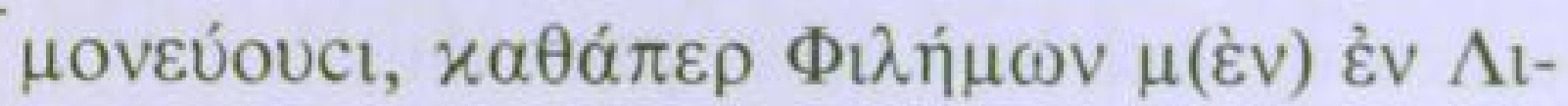
$\theta[0] \gamma \lambda \dot{v} \varphi \omega \iota^{*}$

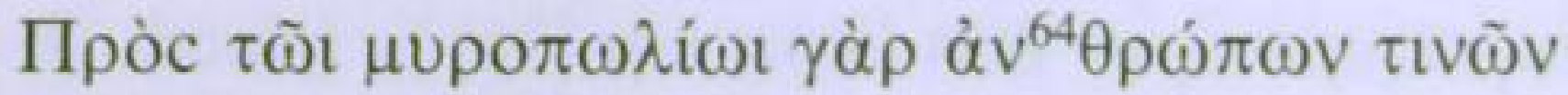

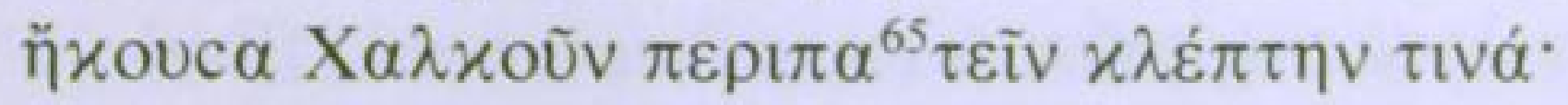

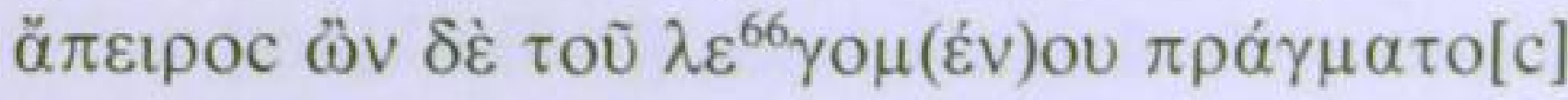
'A 


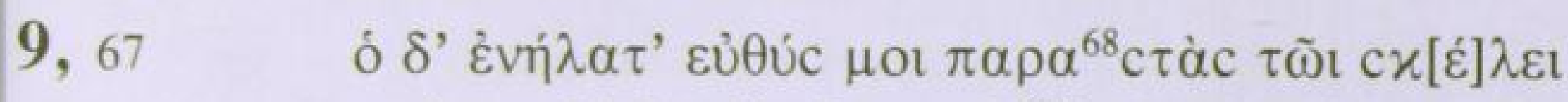

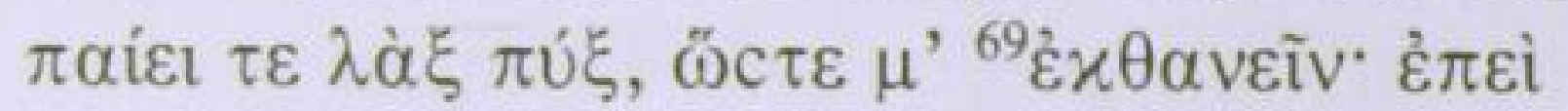

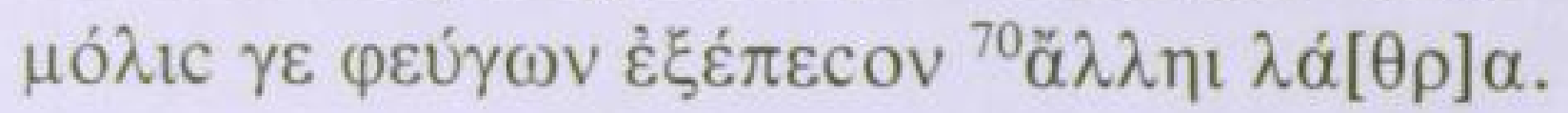

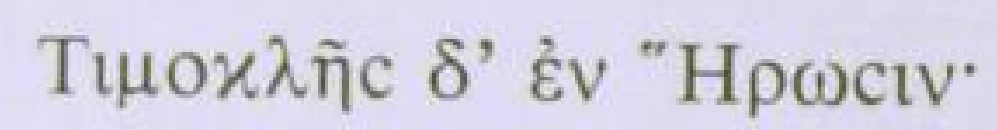

Col. 10

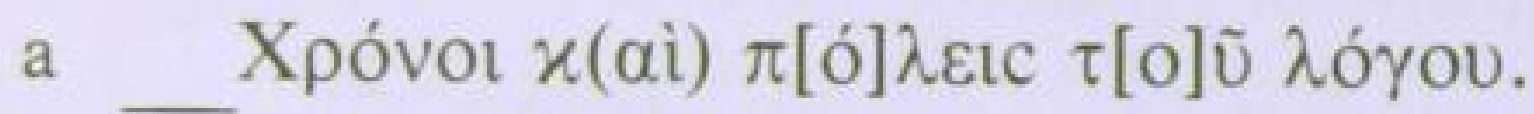

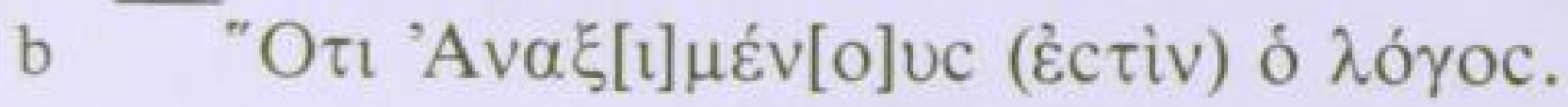

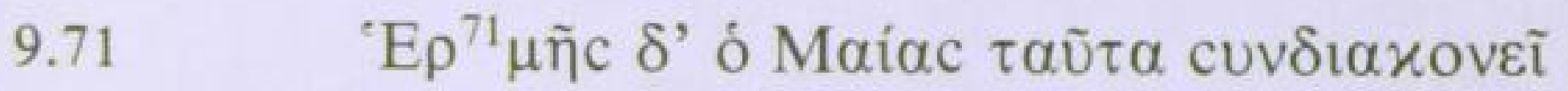

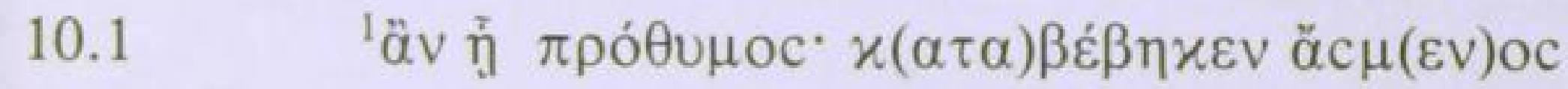

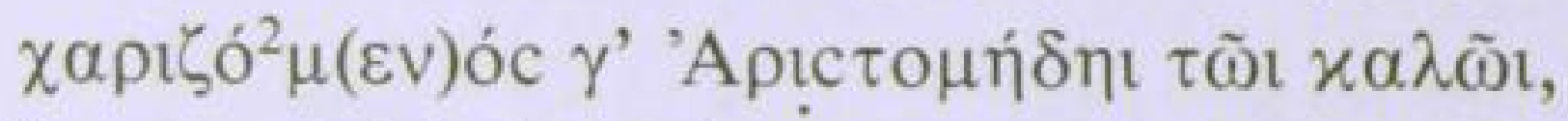

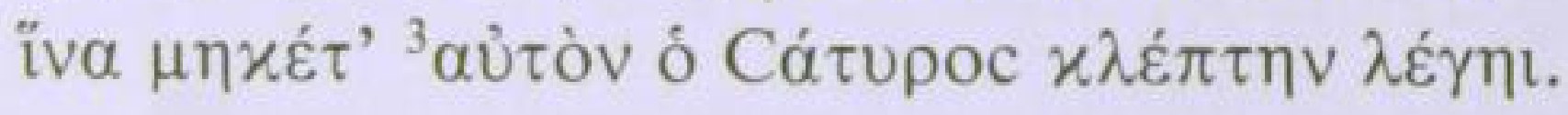
$\chi(\alpha i)$ ह่V 'I-

xapíotc.

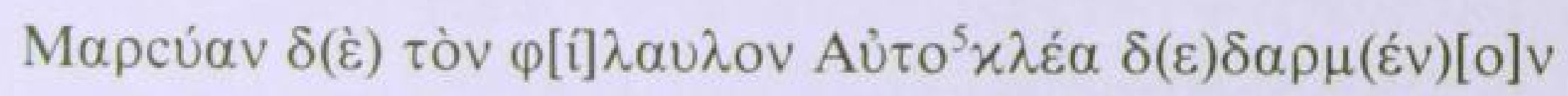

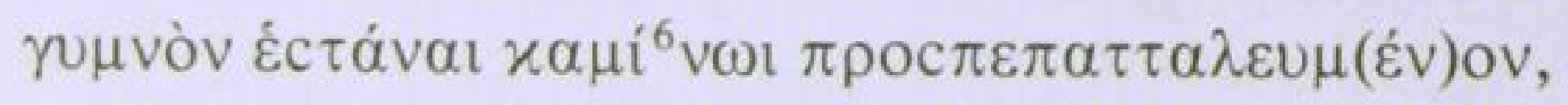




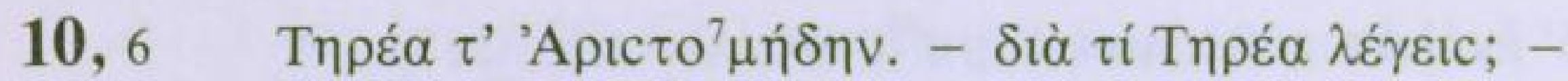

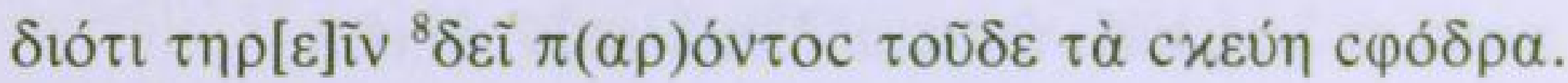

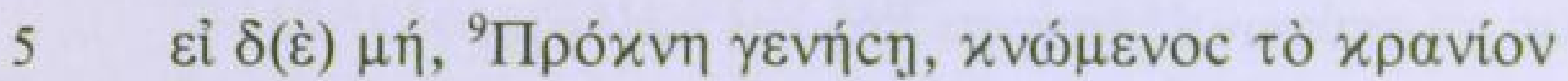

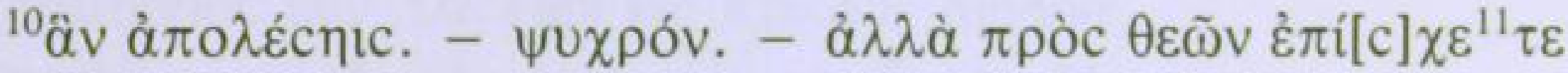
$\mu \eta \delta \varepsilon \dot{~ c u \rho i ́ \xi \eta \tau \varepsilon . ~}$

\section{[I]A}

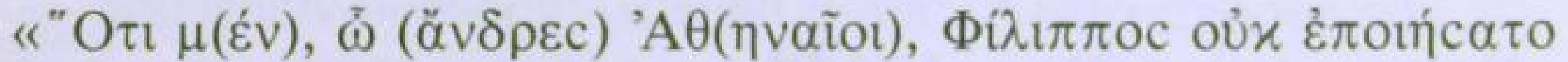

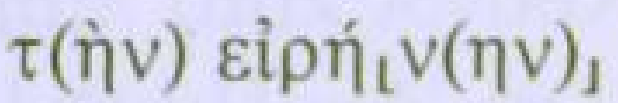

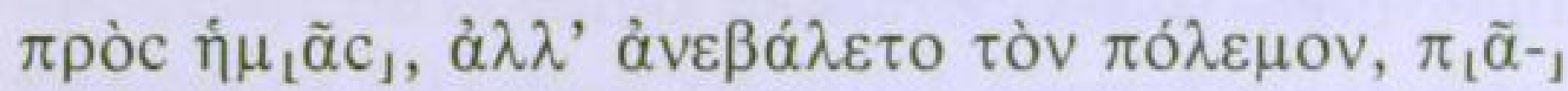

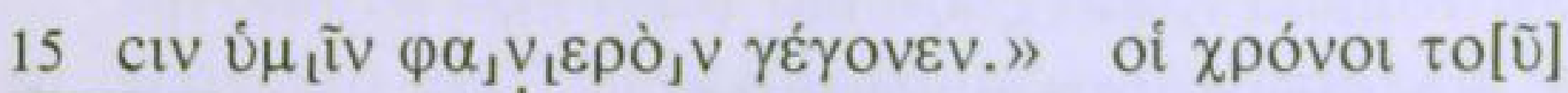

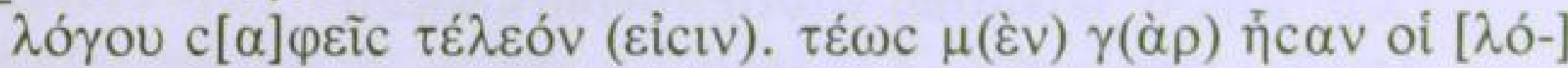

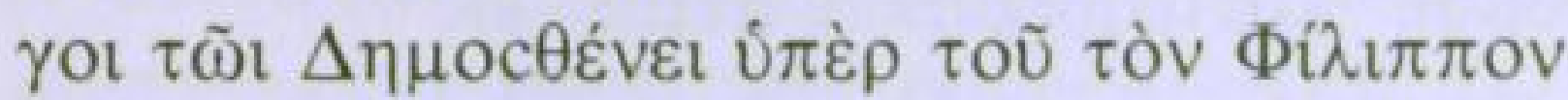

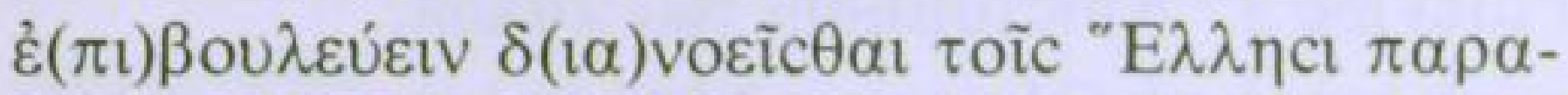

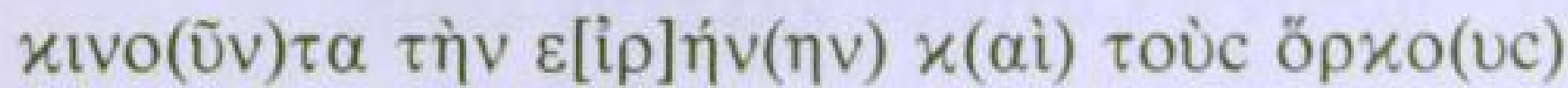

$$
\pi(\alpha \rho \alpha) \beta \alpha i v o[v-]
$$

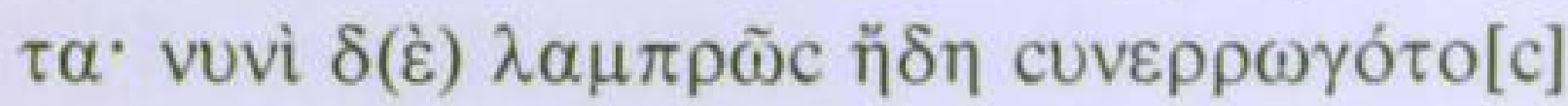

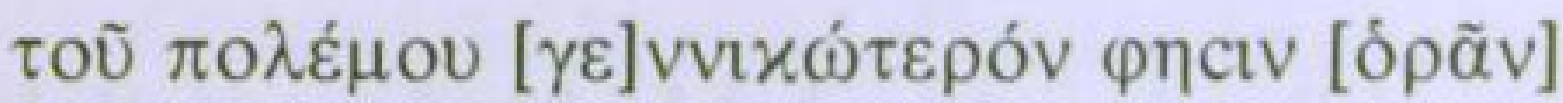

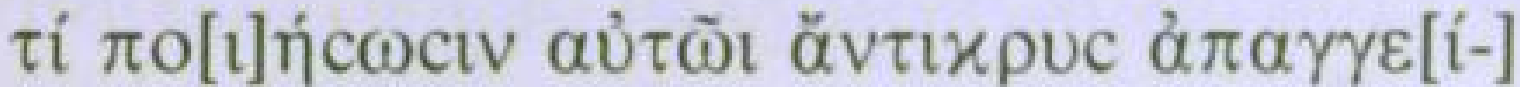

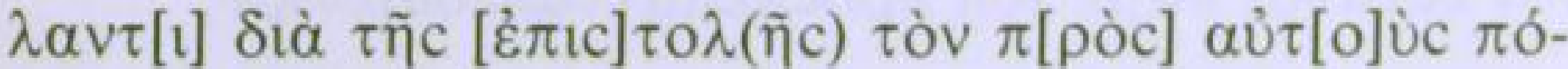




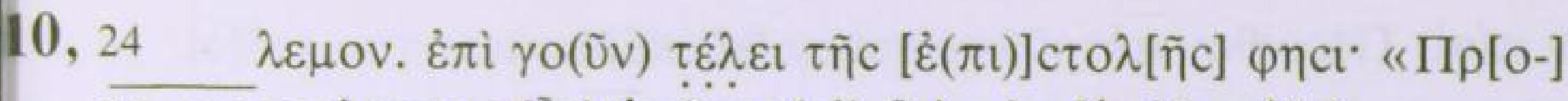

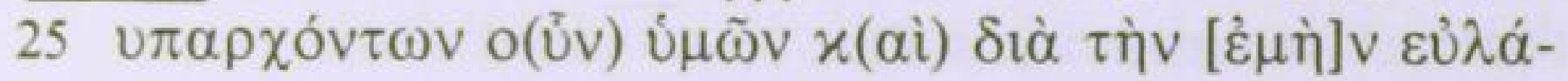

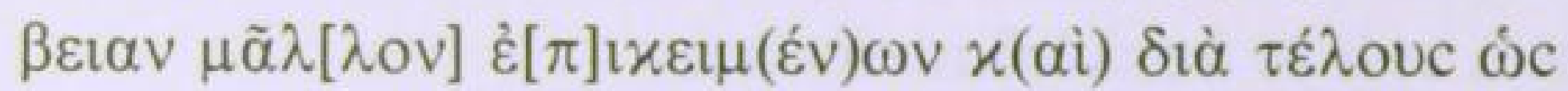

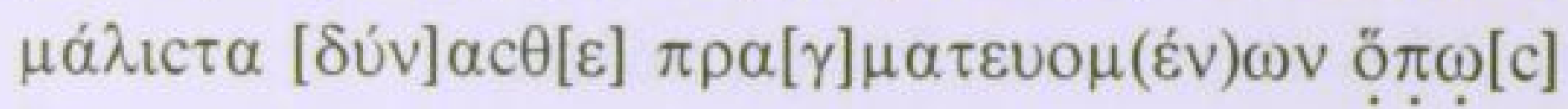

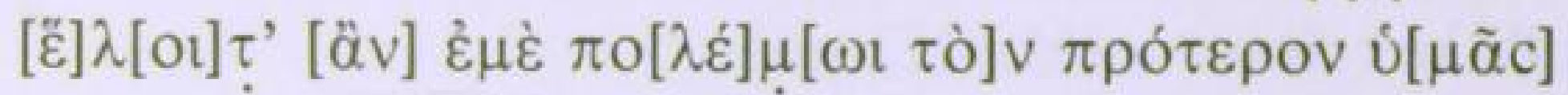

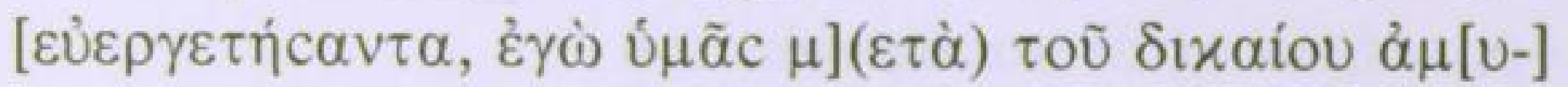

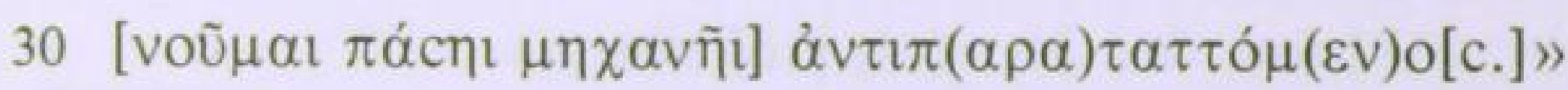

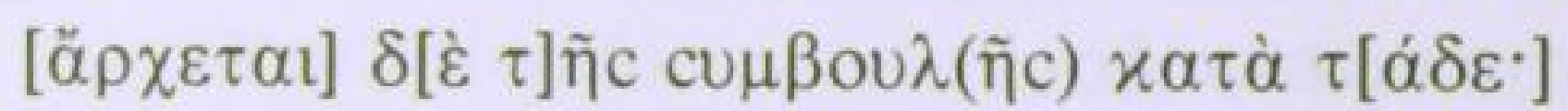

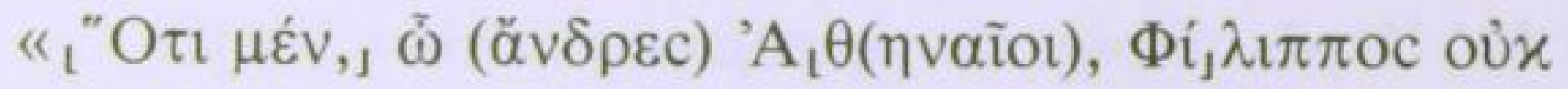

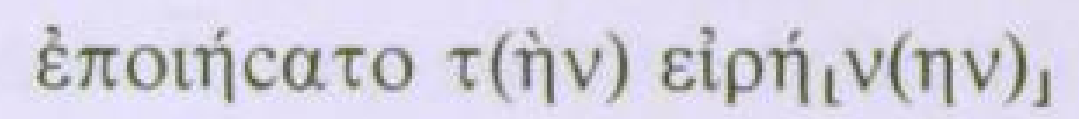

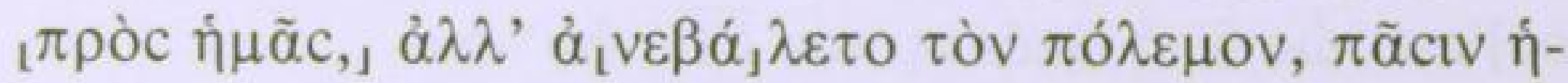

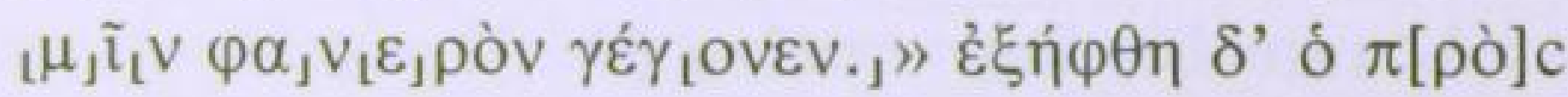

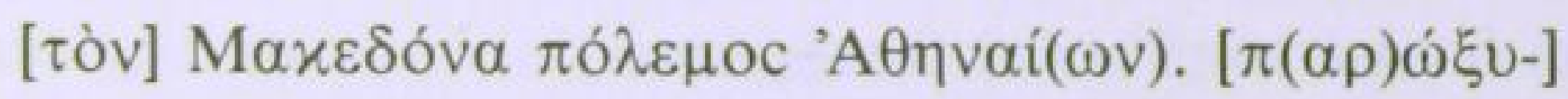

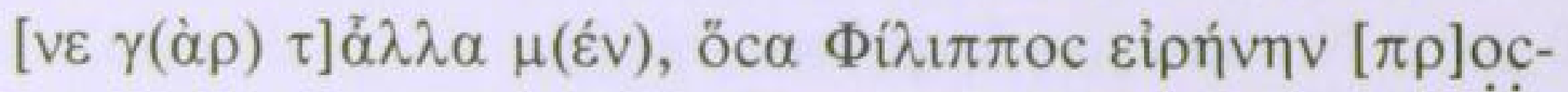

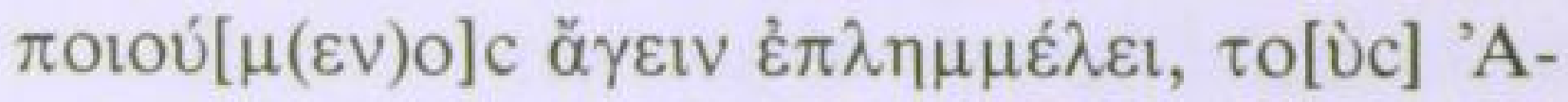

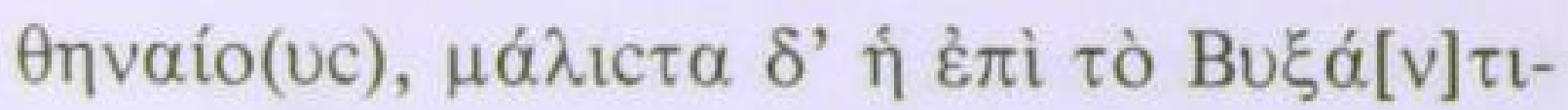




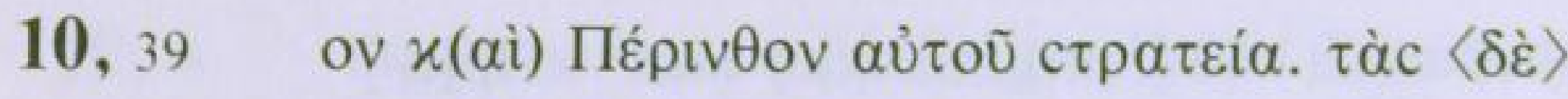

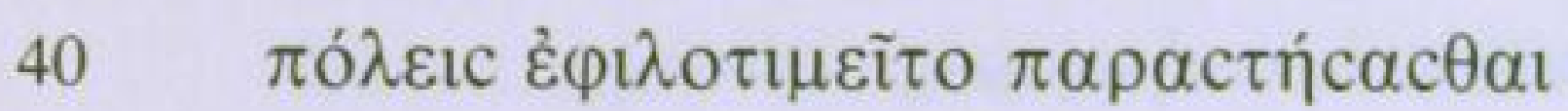

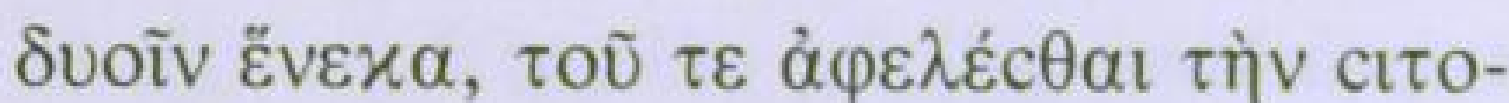

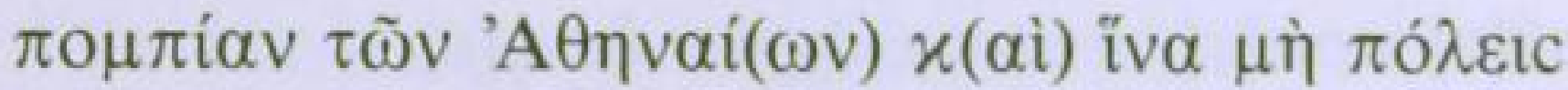

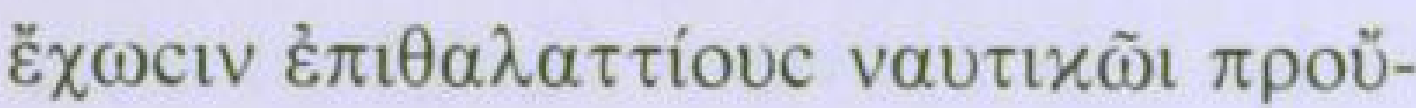

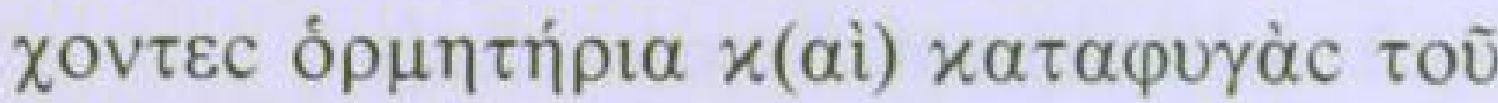

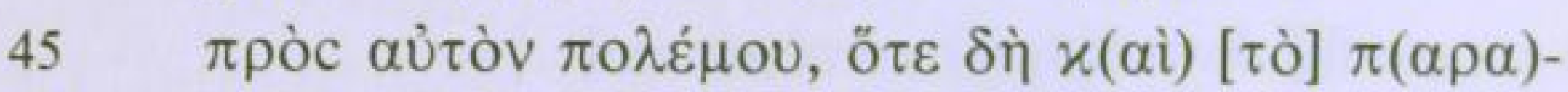

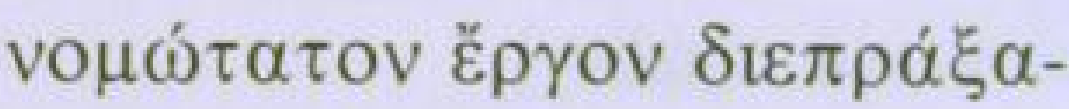

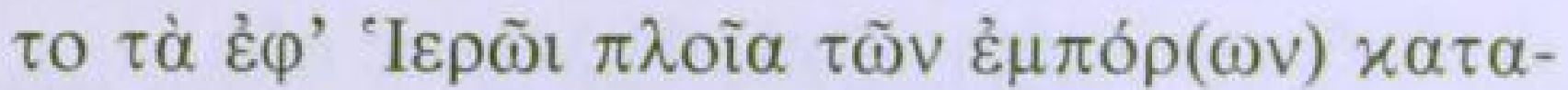

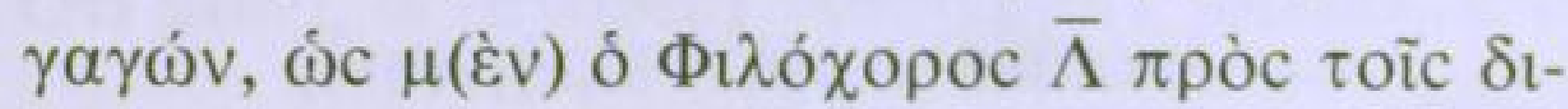

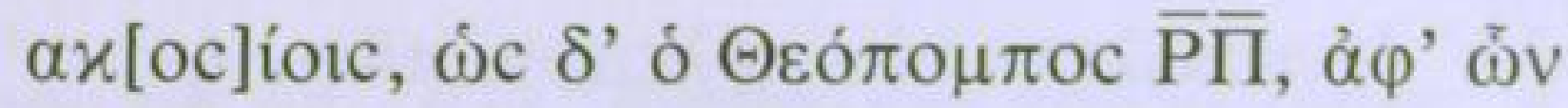

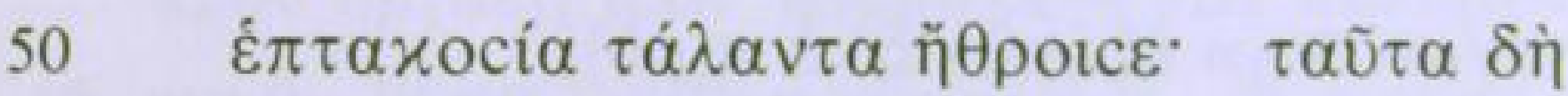

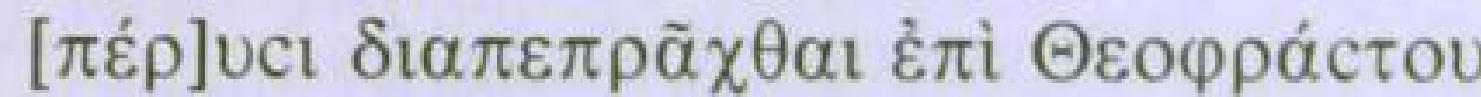

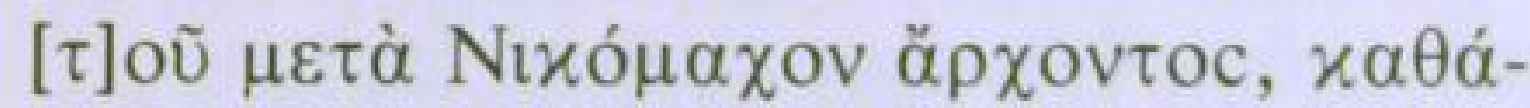

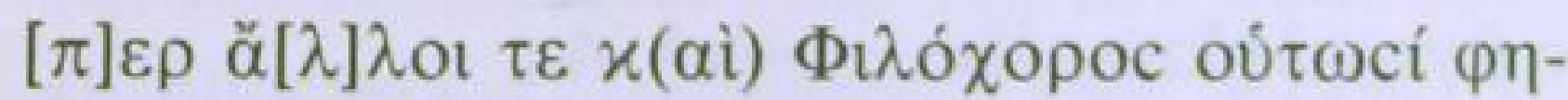

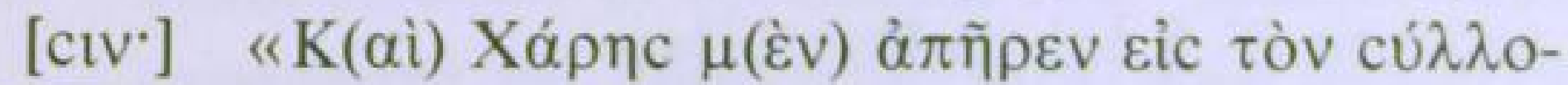
$55 \gamma[0 v] \tau \tilde{\omega} v \beta \alpha c \iota \lambda \iota x(\tilde{\omega} v) c \tau \rho \alpha \tau \eta \gamma \tilde{\omega} \vee x \alpha \tau \alpha \lambda_{1}-$

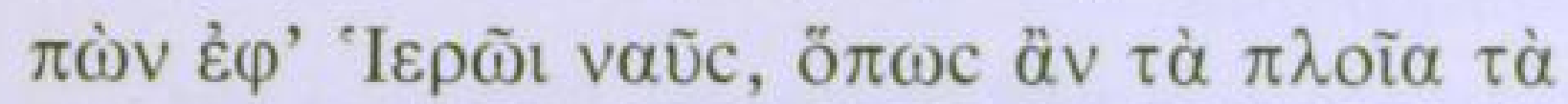

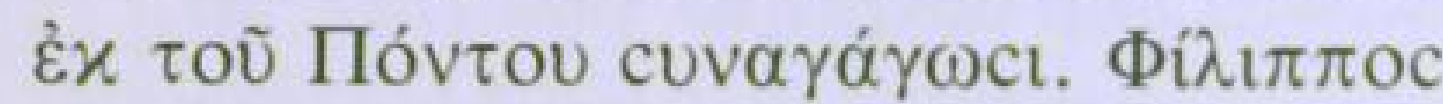

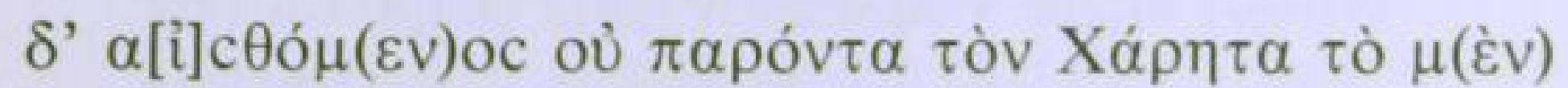

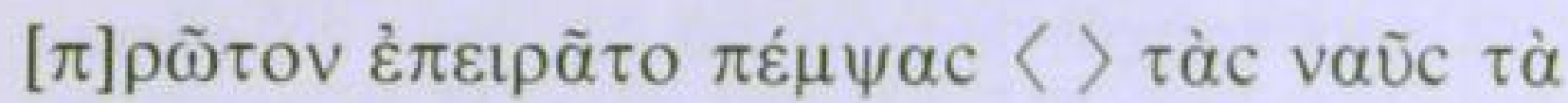

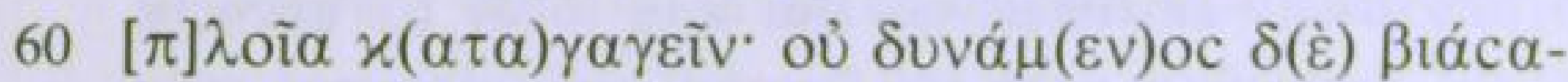




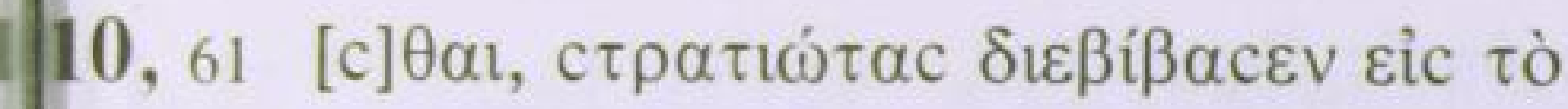

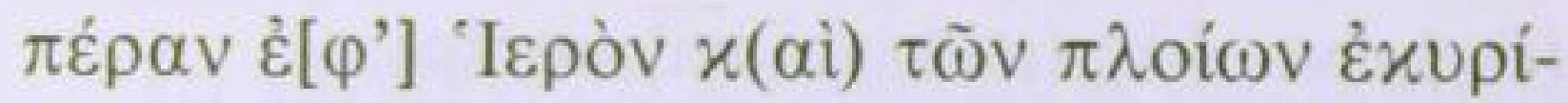

\section{Col. 11}
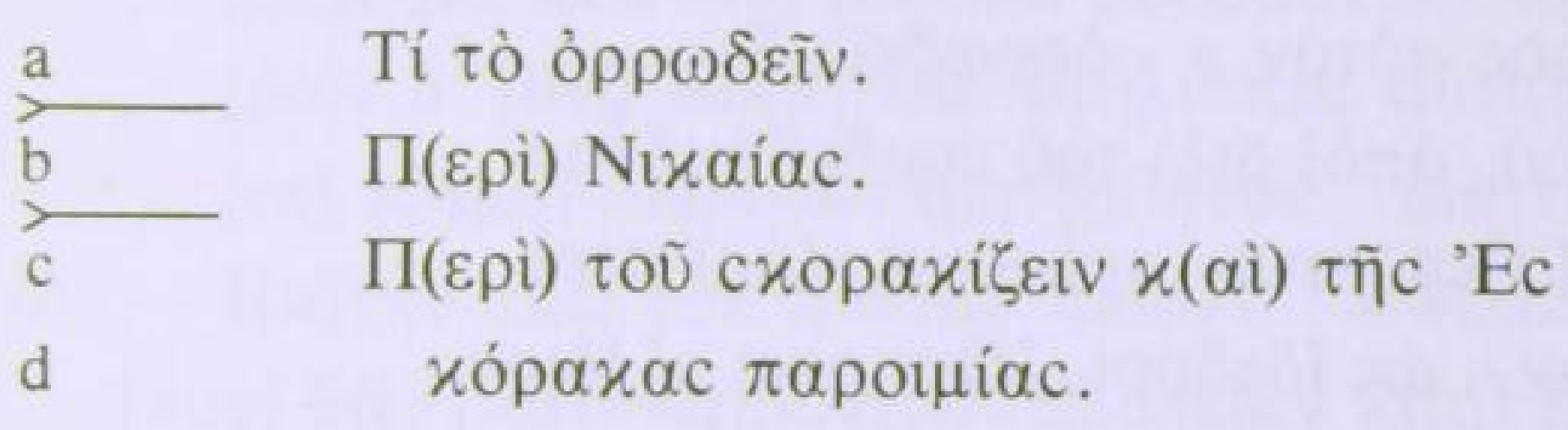

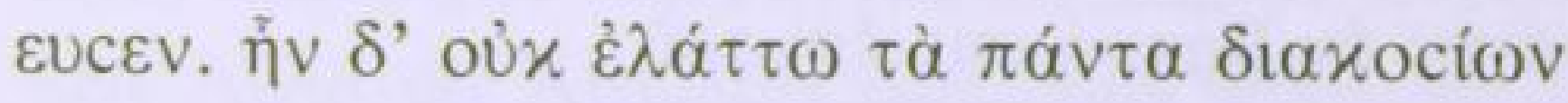

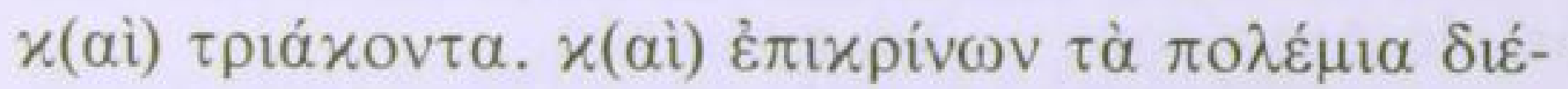

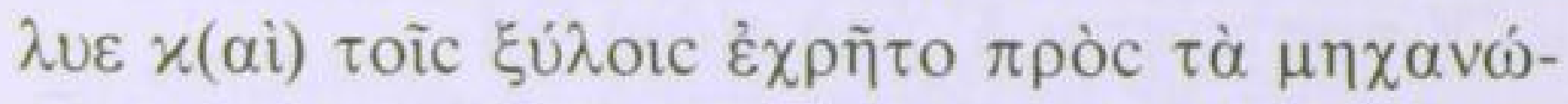
$\mu \alpha \tau \alpha, x(\alpha i)$ cítov $x(\alpha i) \beta v \rho c \tilde{v} v x(\alpha i) \chi \rho \eta \mu \alpha \dot{\tau} \omega v \pi \mathrm{o} \lambda$ -

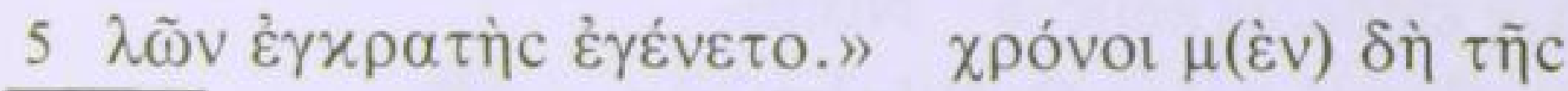

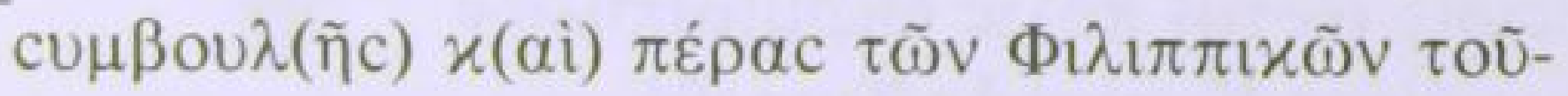

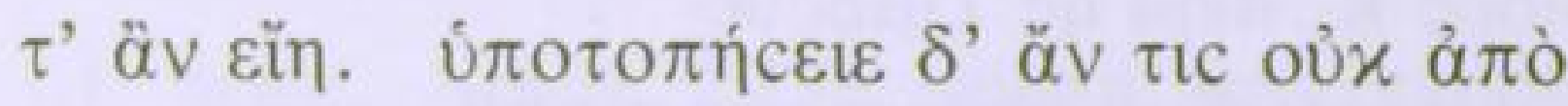

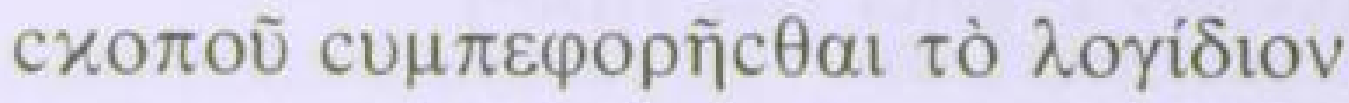

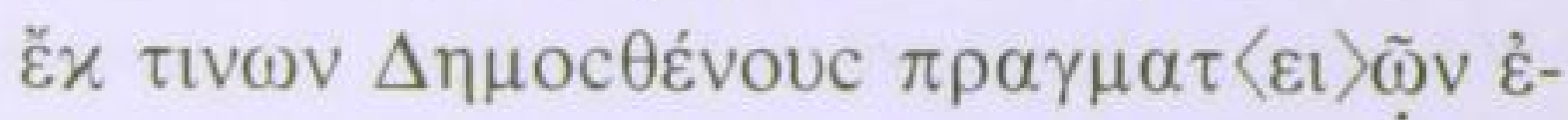

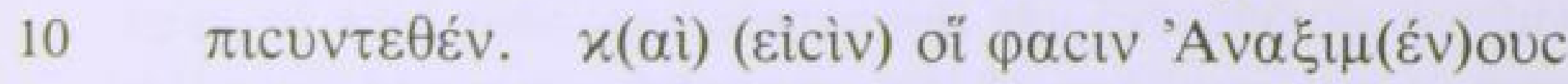

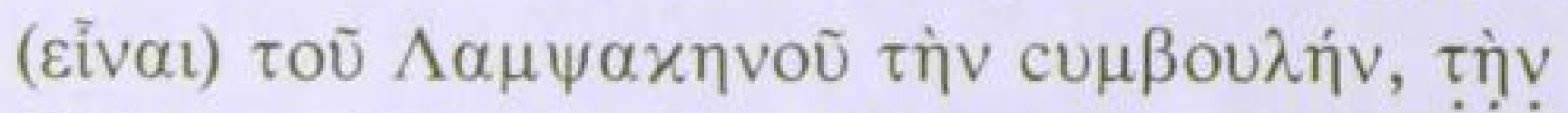

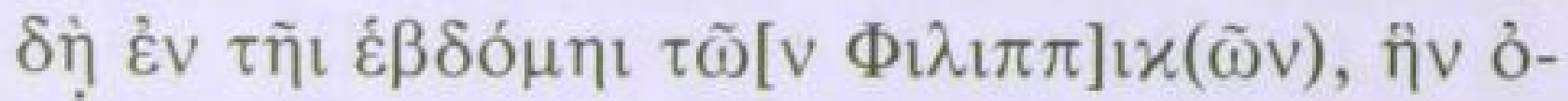




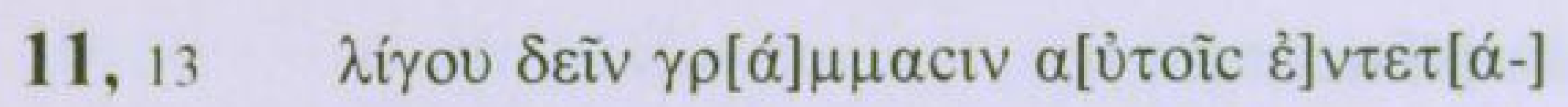

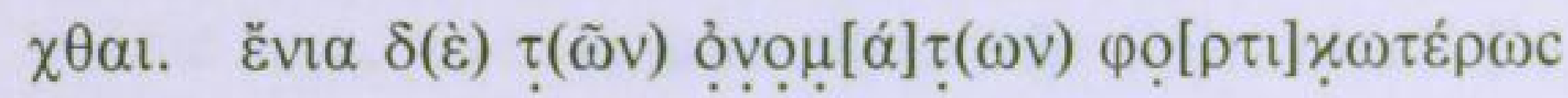

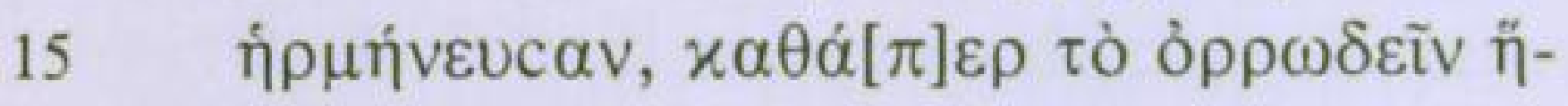

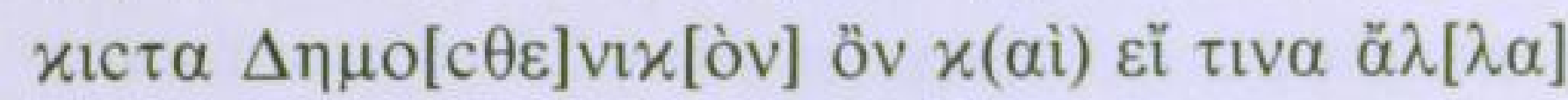

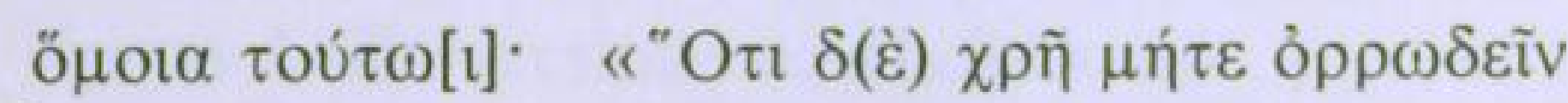

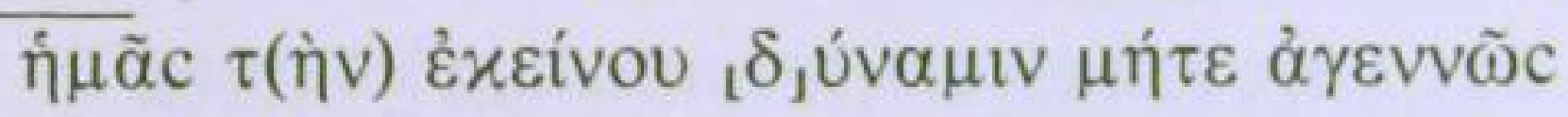

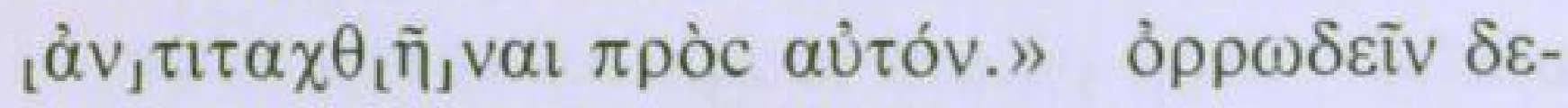

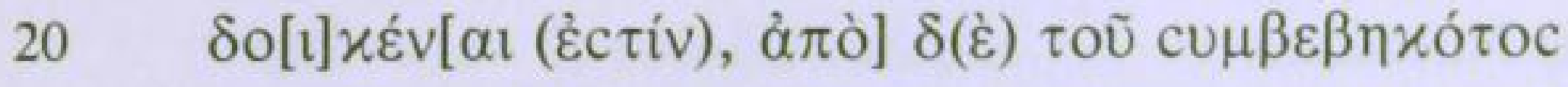

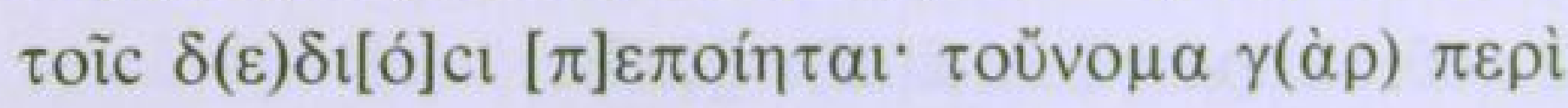

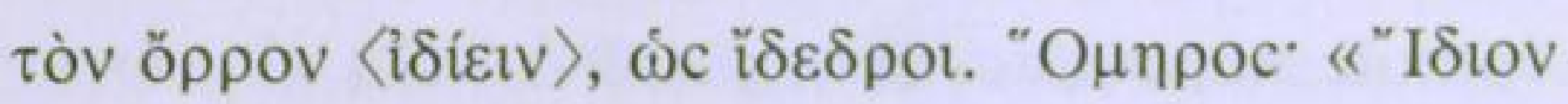

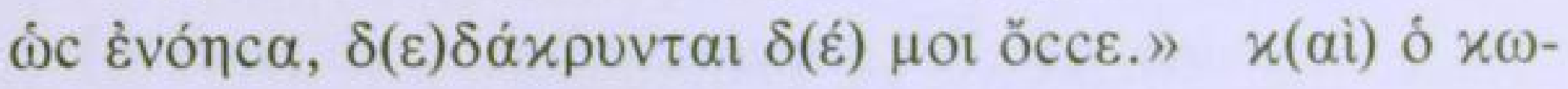

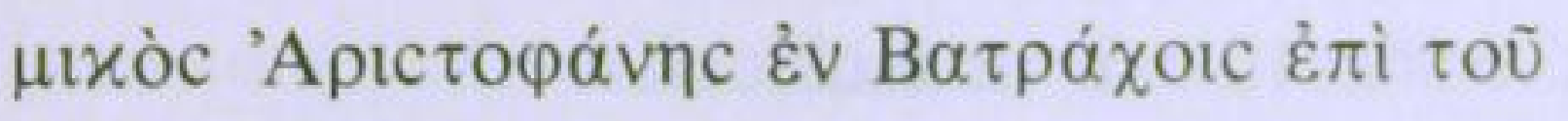

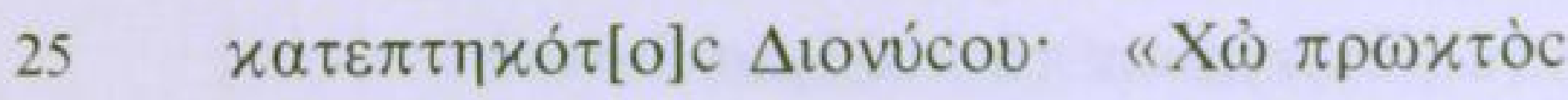

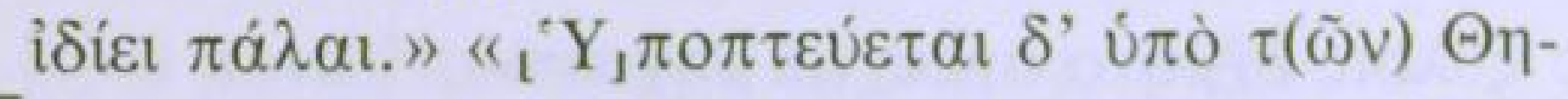

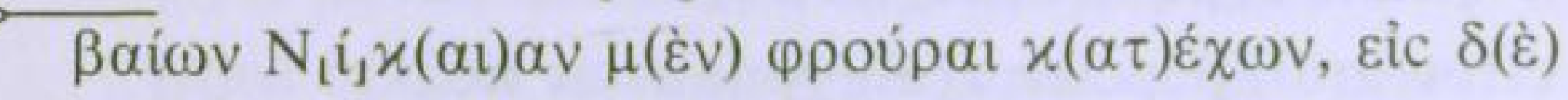

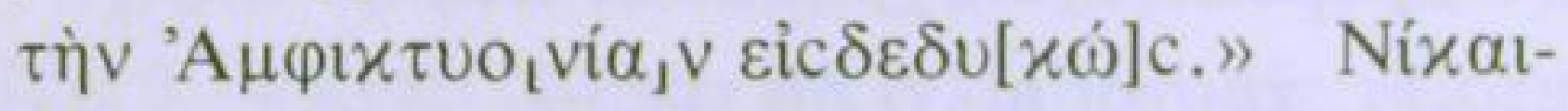

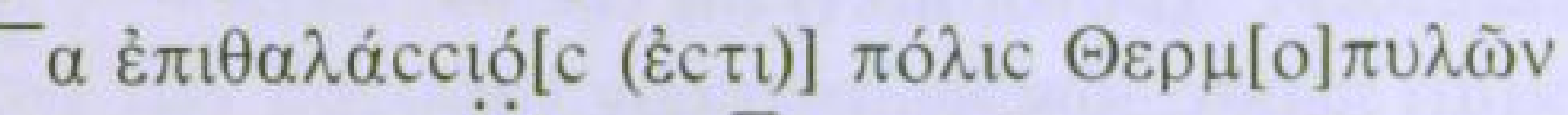

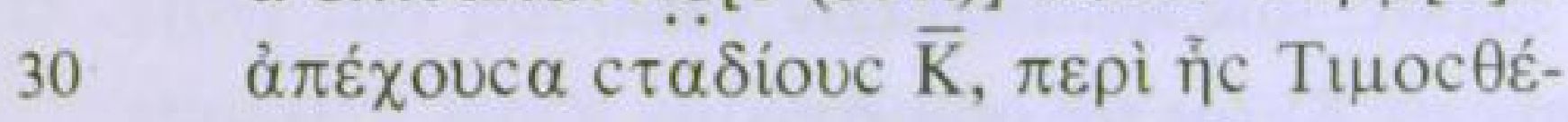

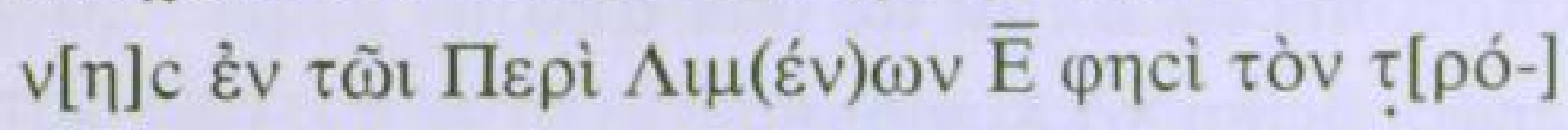


11,32 [

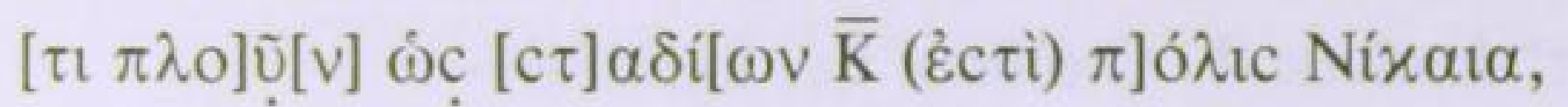

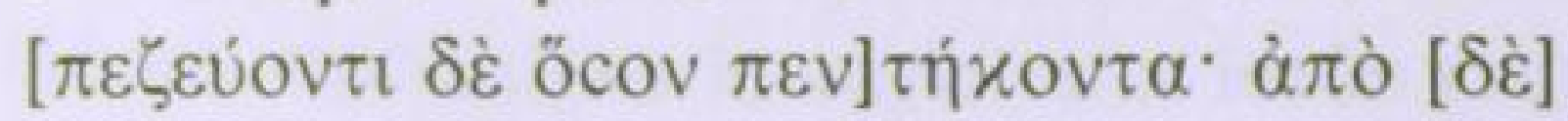

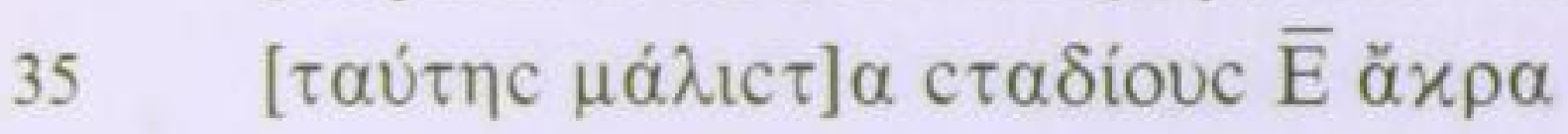

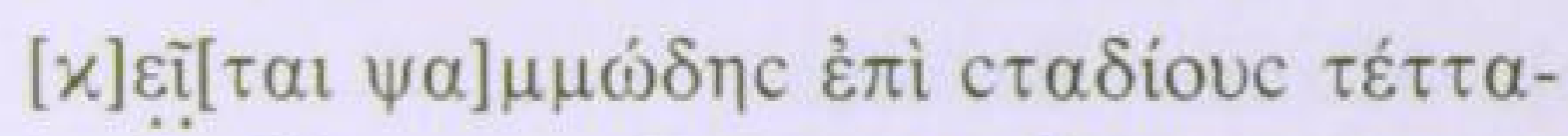

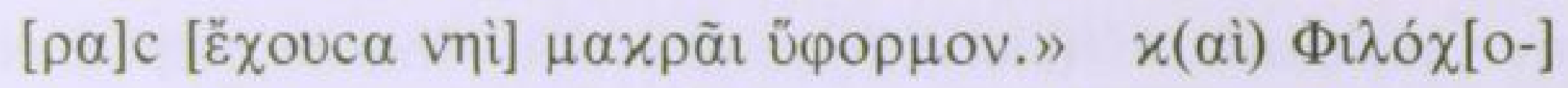

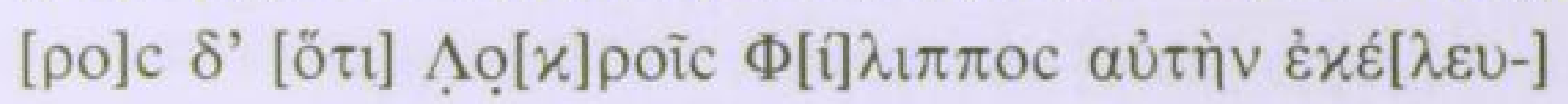

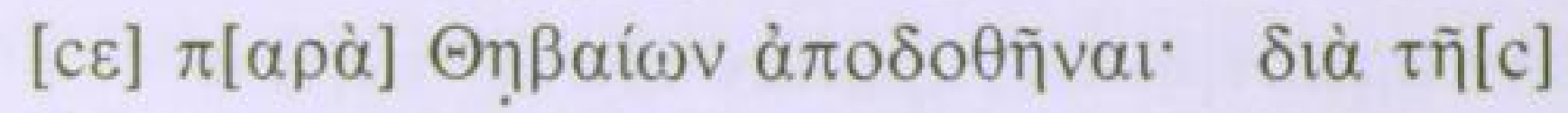

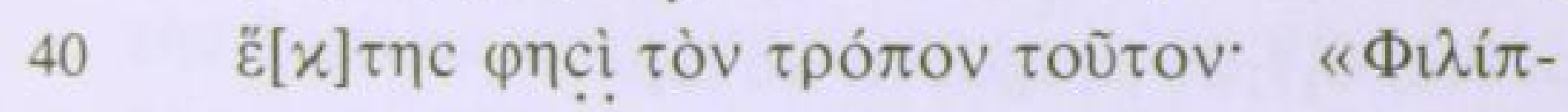

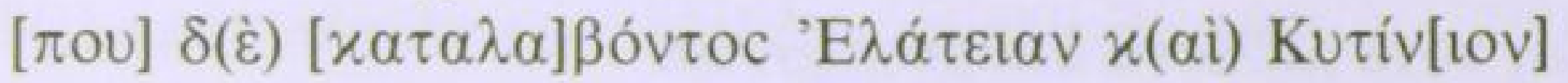

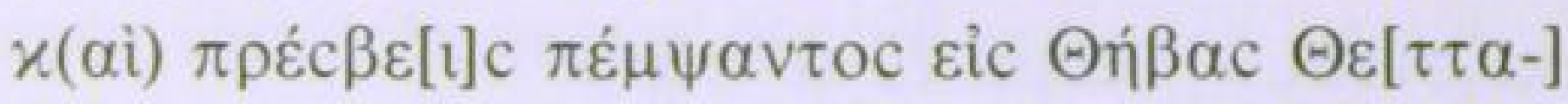

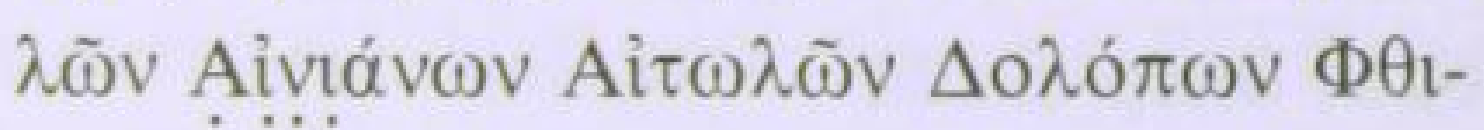

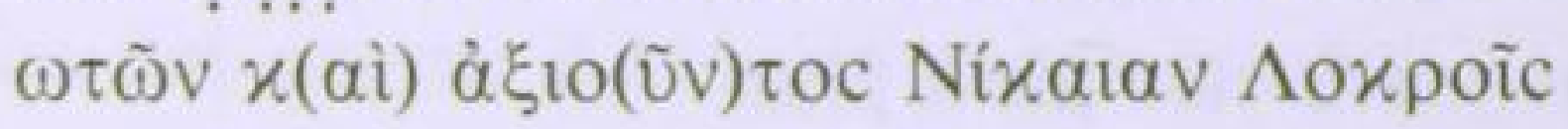

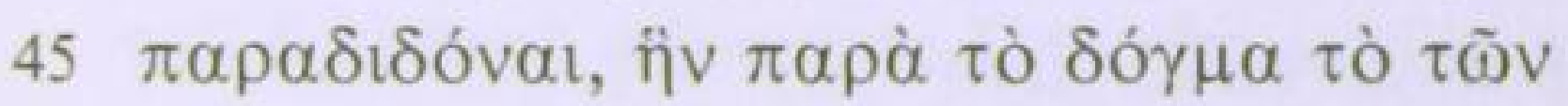




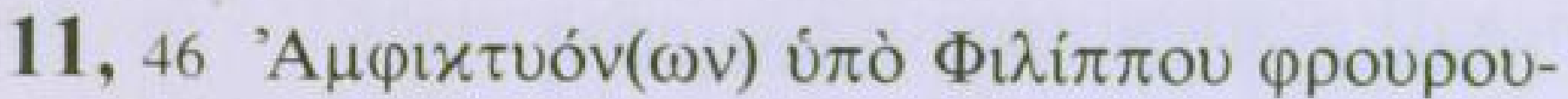

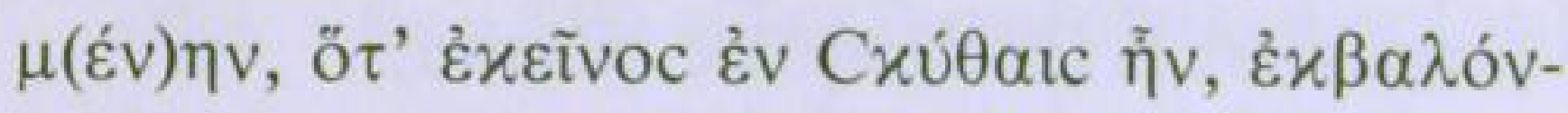

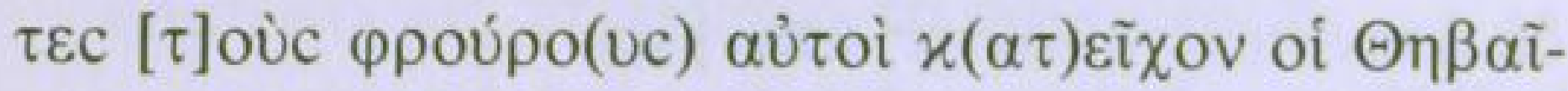

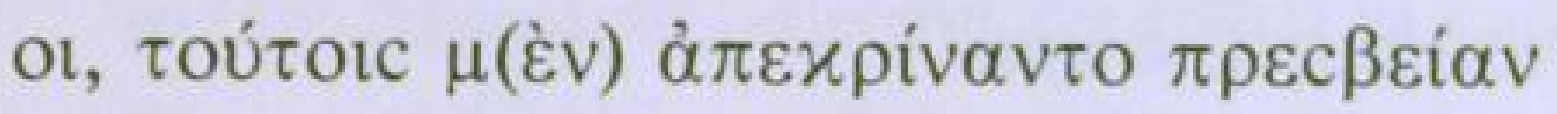

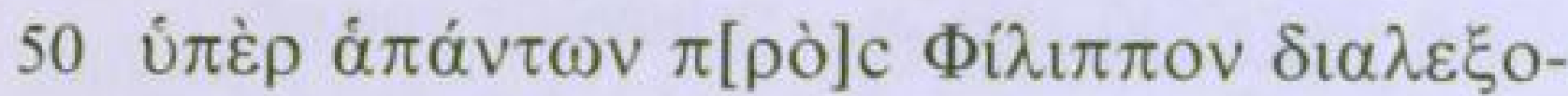

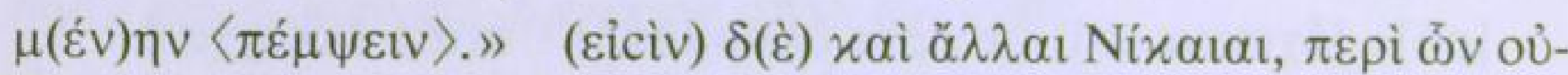

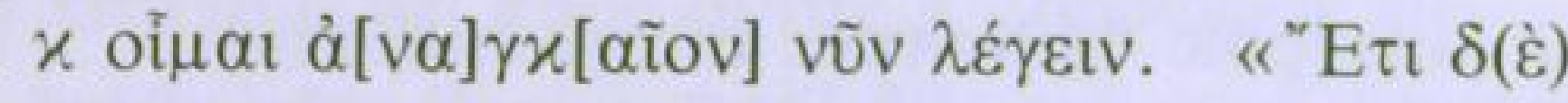

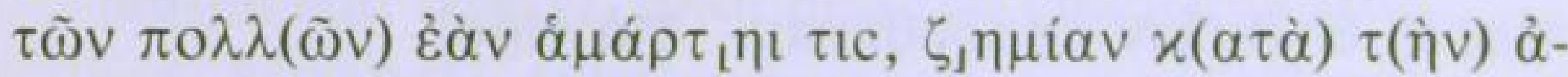

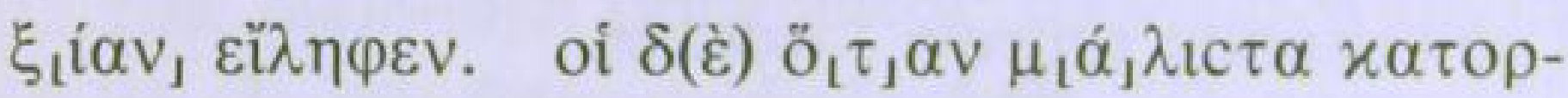

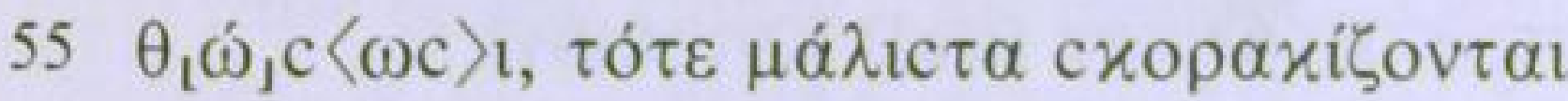

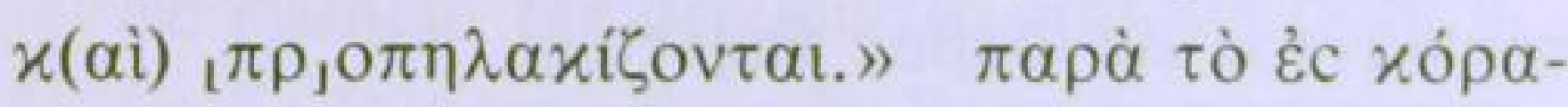

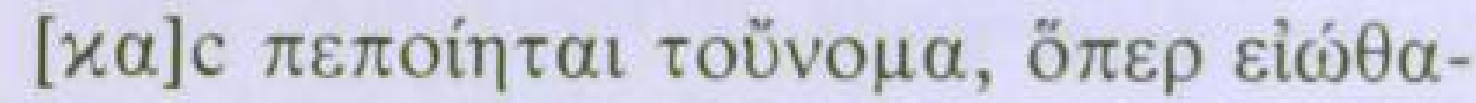

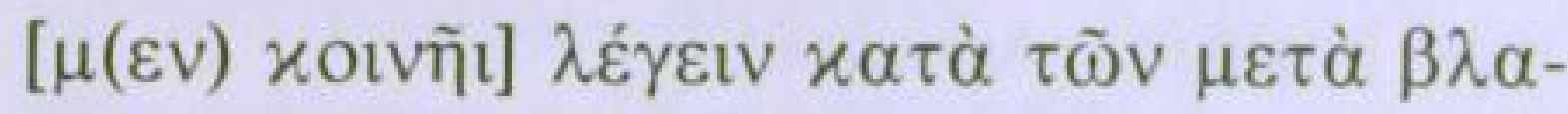

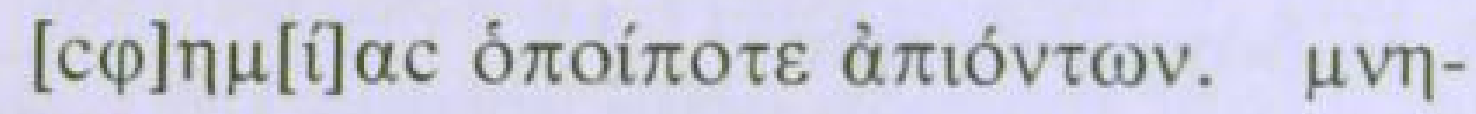

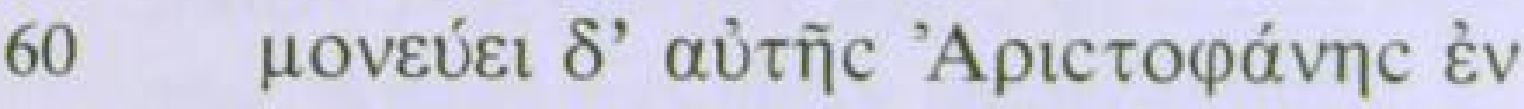

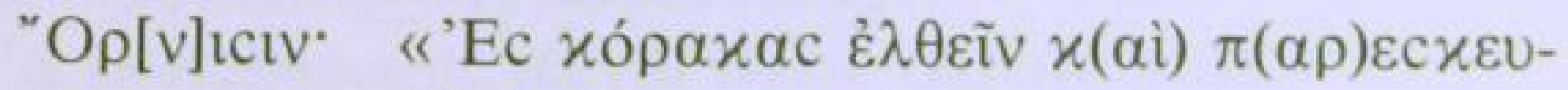

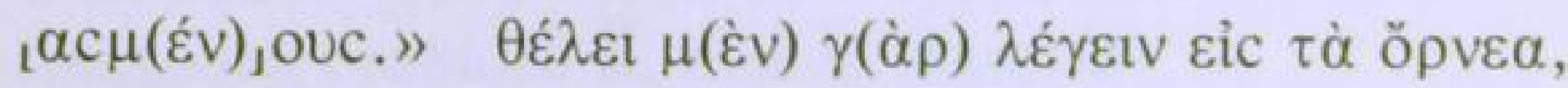

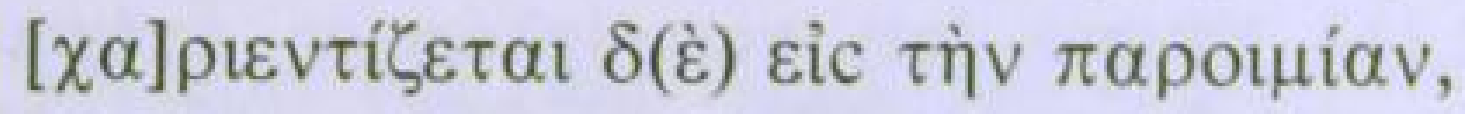

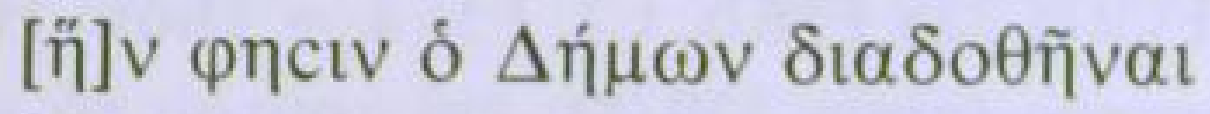

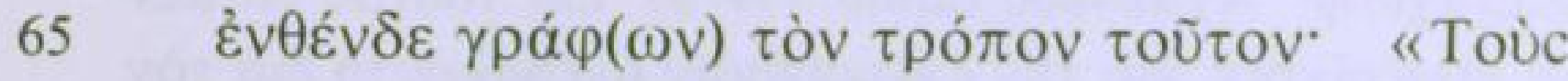

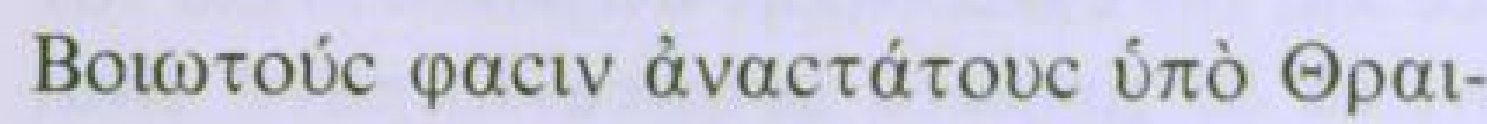


Col. 12

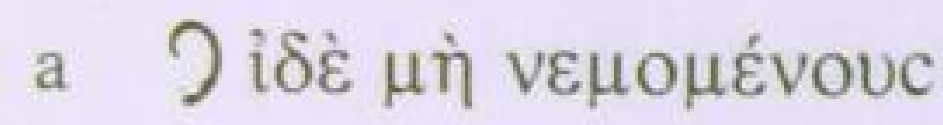

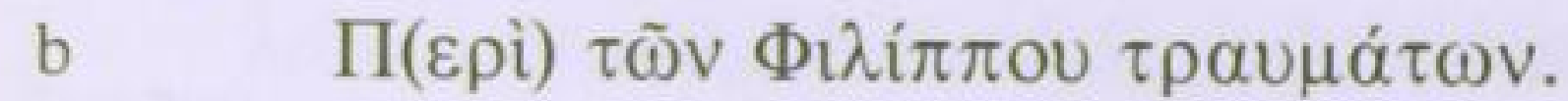

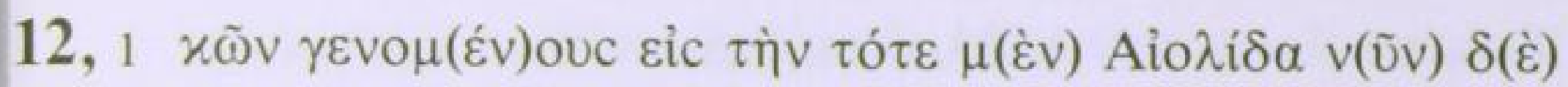

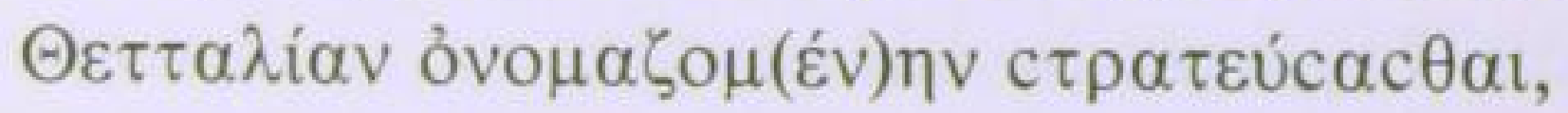

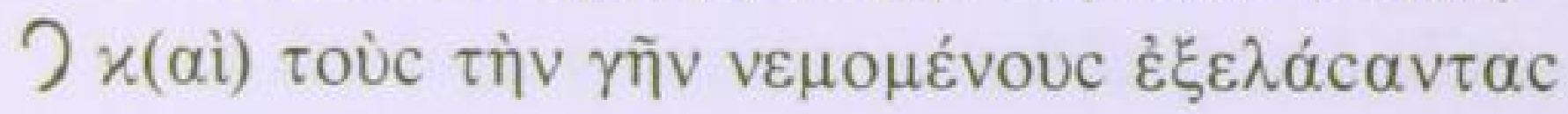

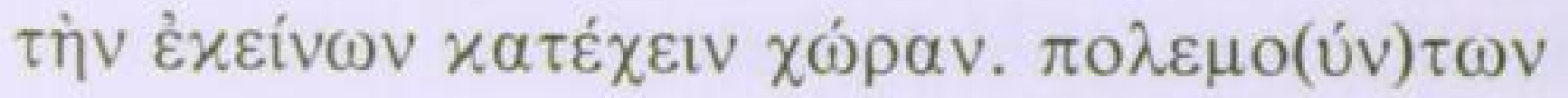

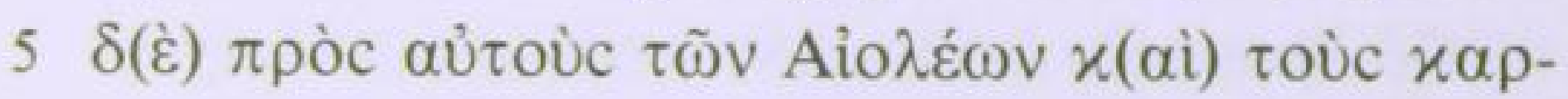

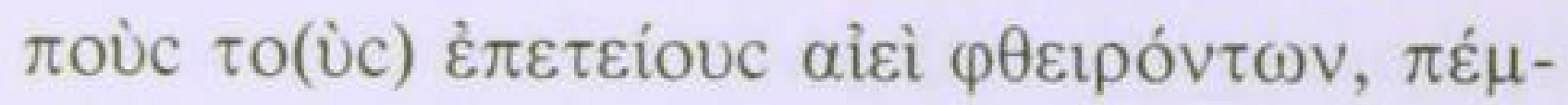

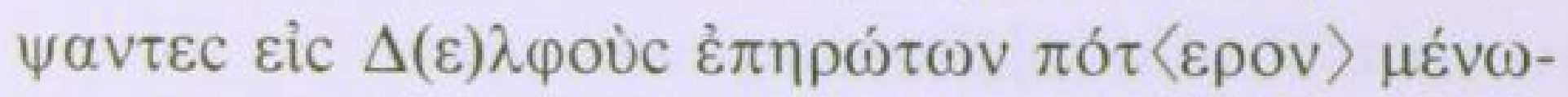

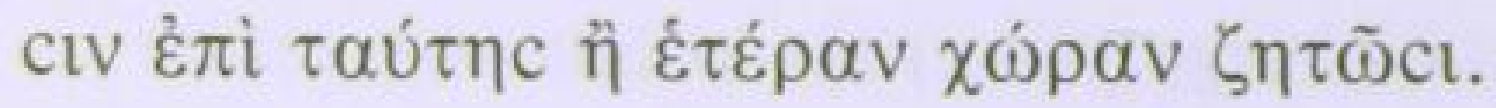

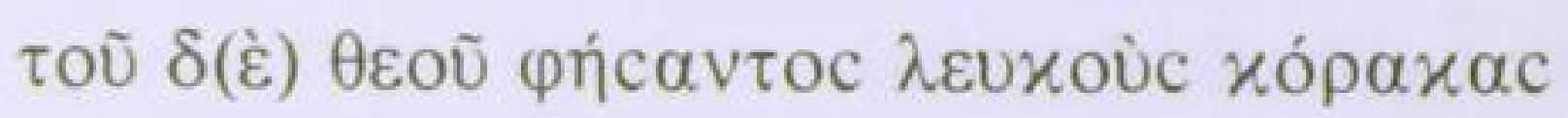

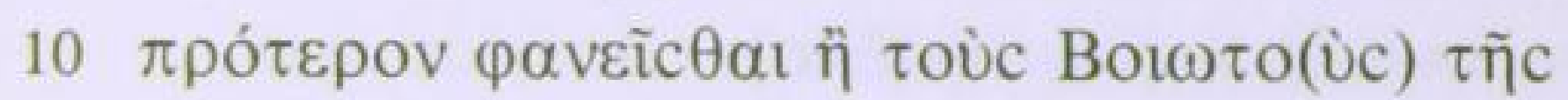

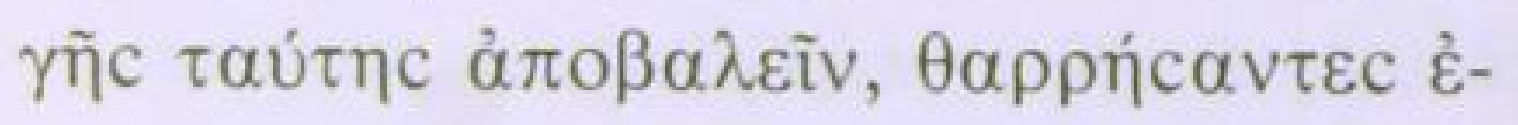

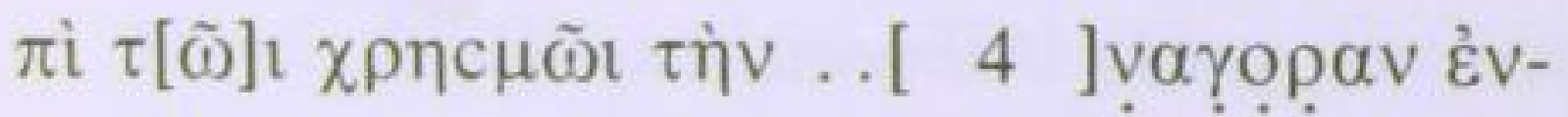

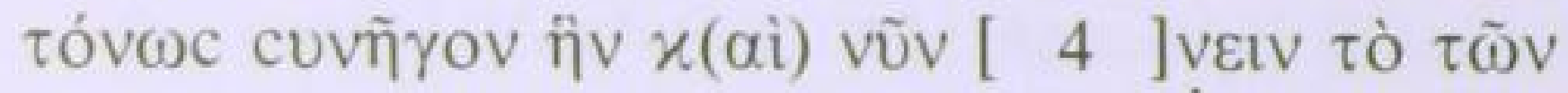

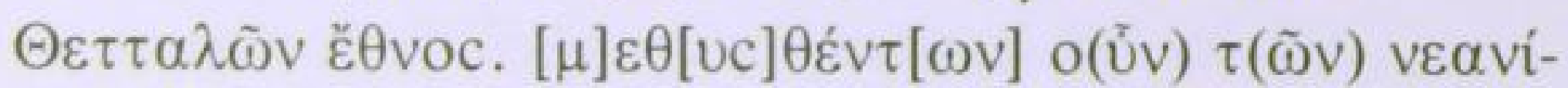

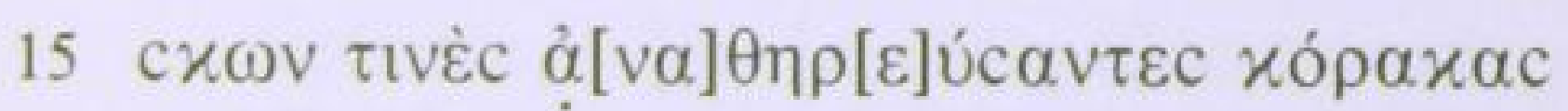

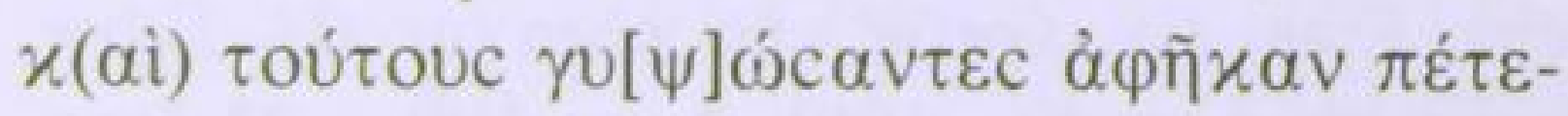




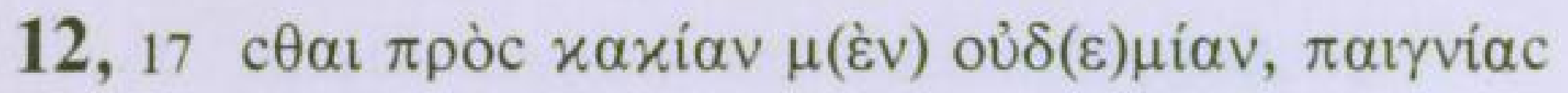

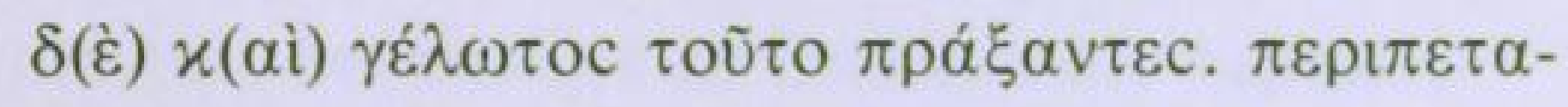

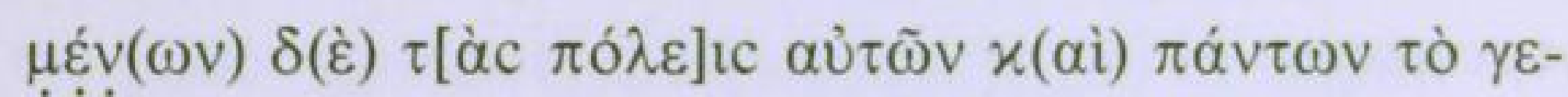

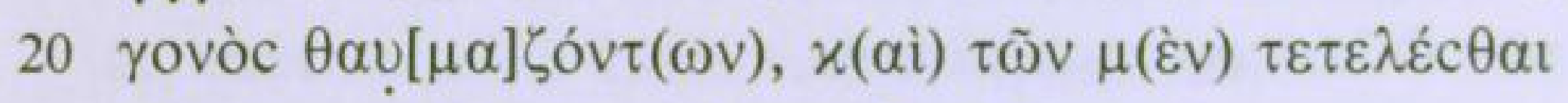

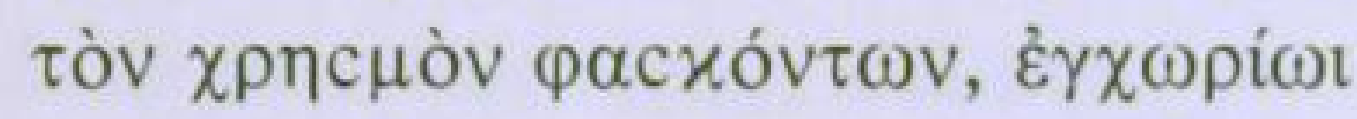

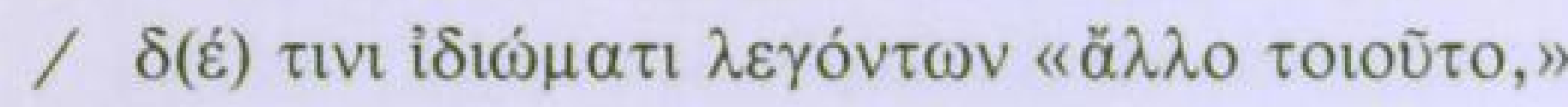

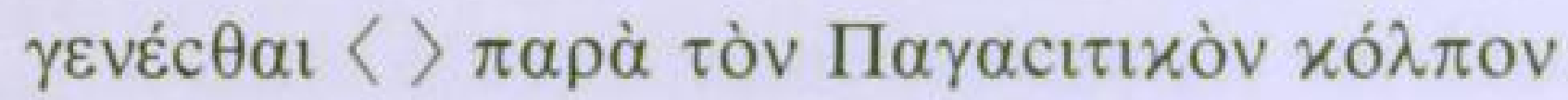

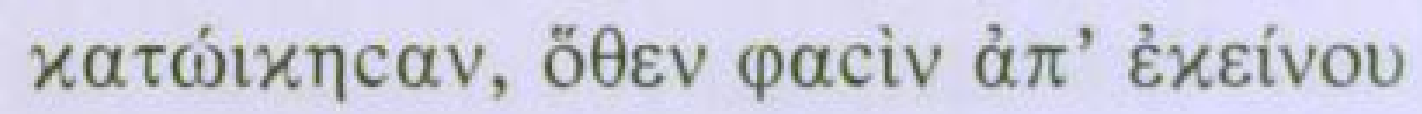

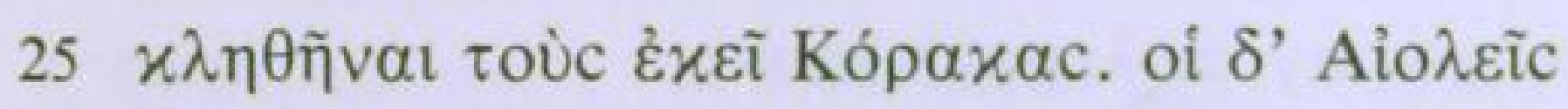

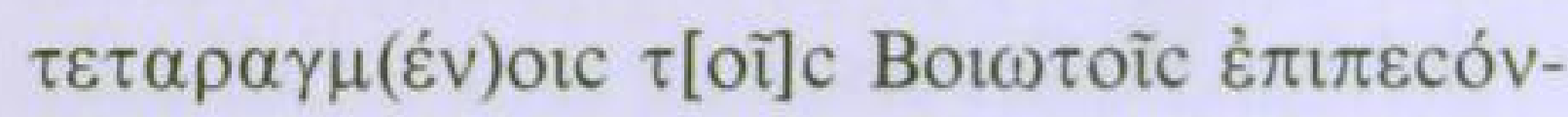

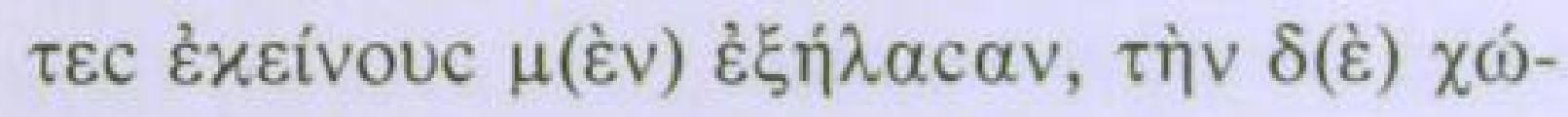

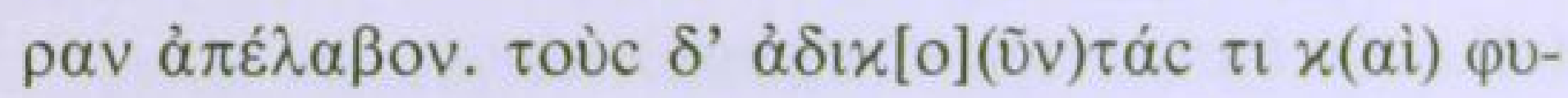

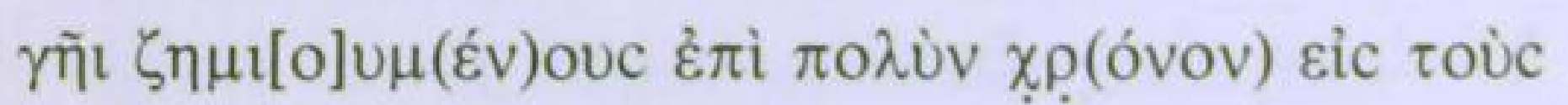

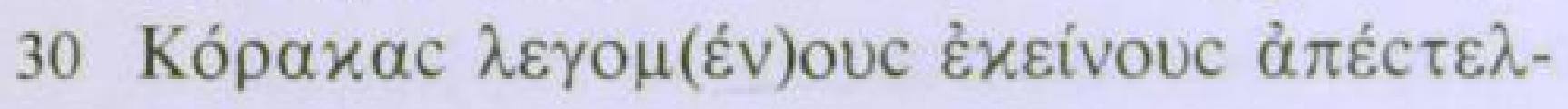

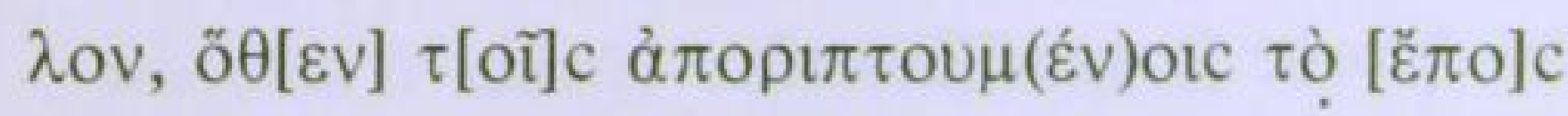

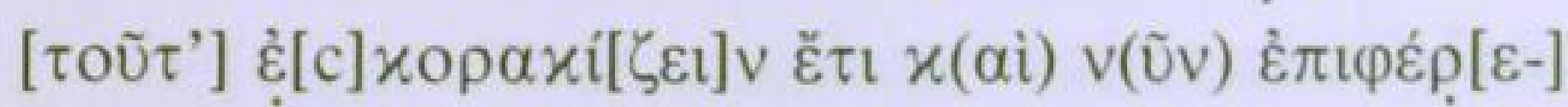

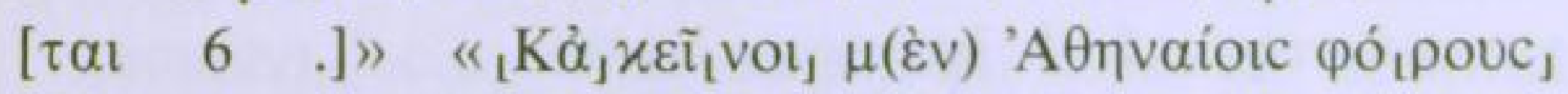




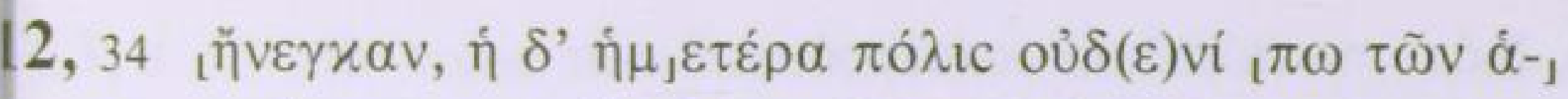

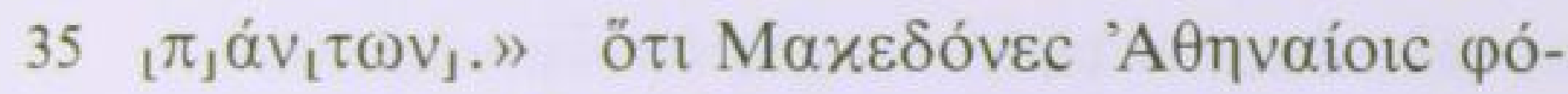

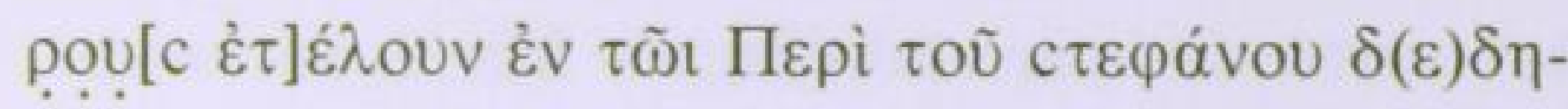

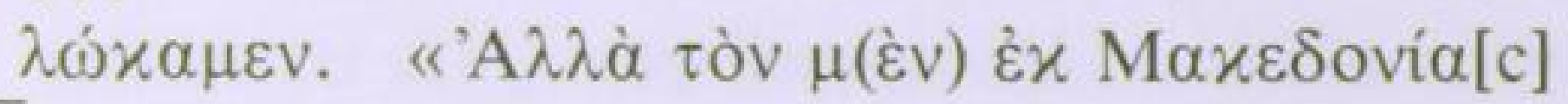

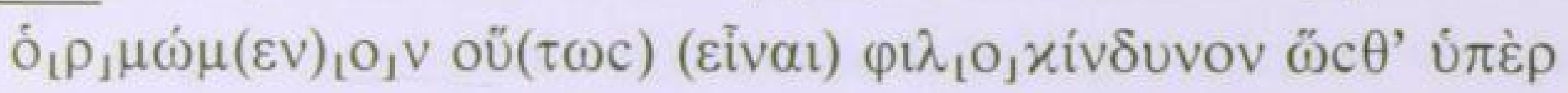

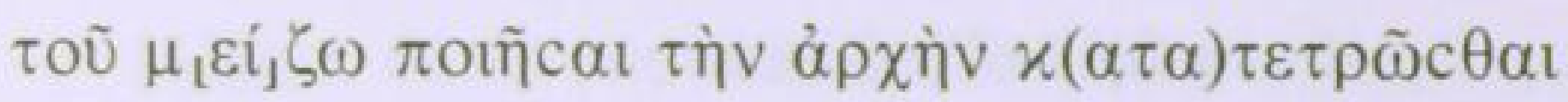

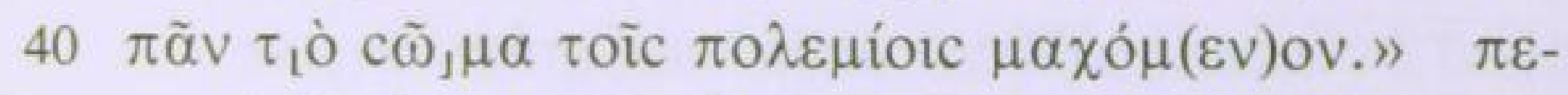

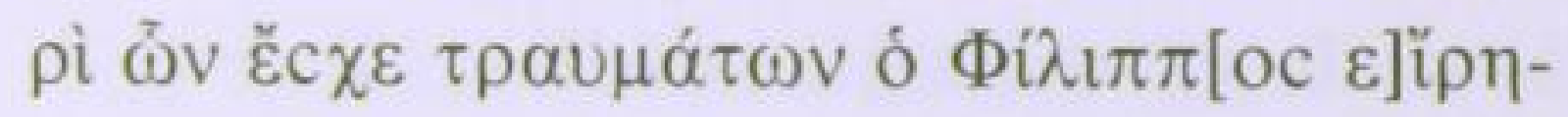

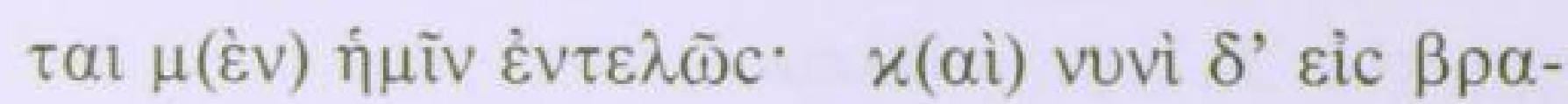

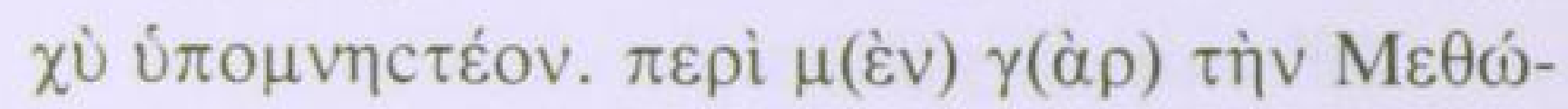

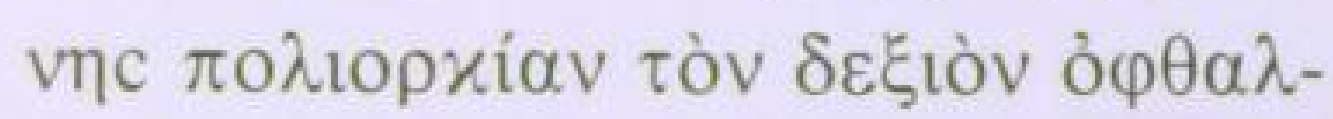

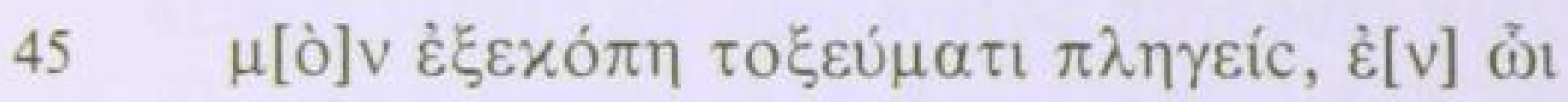

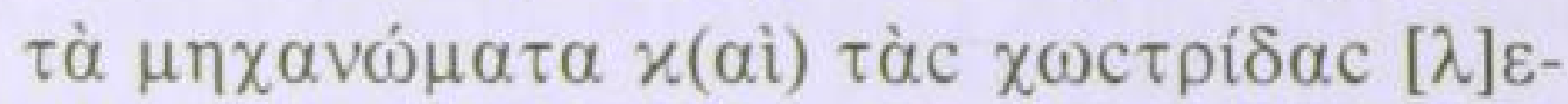

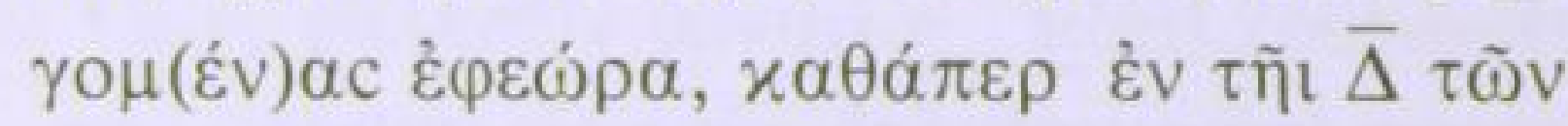

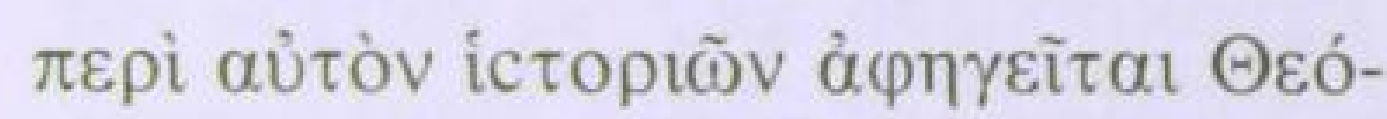

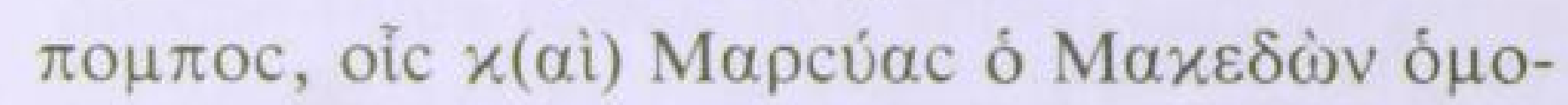

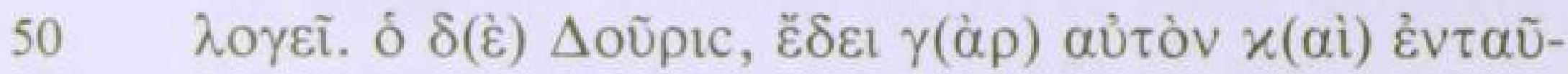

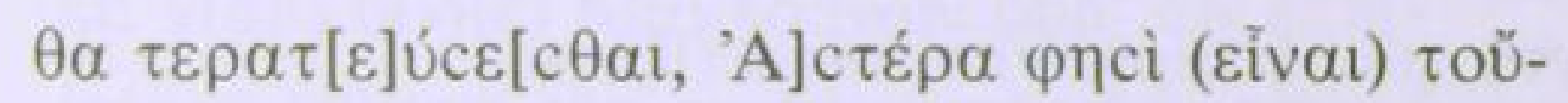

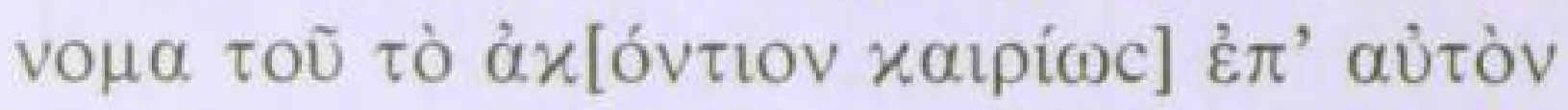

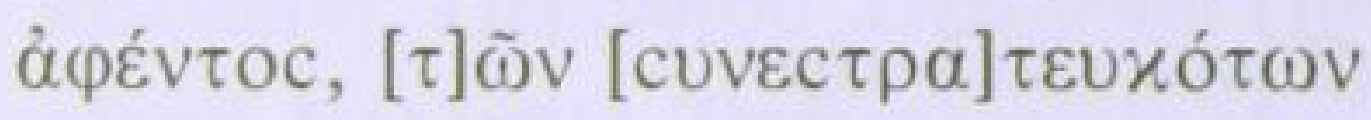

$\alpha u ̛ \tau \tilde{\iota} \mathrm{c} \chi \varepsilon[\delta]$ òv $[\pi \alpha \dot{v}] \tau \omega \nu \tau o \xi \xi \varepsilon \dot{\mu} \alpha[\tau] \mathrm{l}$

$55 \lambda \varepsilon \gamma o ́ v \tau \omega v[\alpha] \cup ̉ \tau o ̀[v] \tau \varepsilon \tau \rho \tilde{c} \theta \alpha$ l. $\tau \dot{\alpha} \mu(\grave{\varepsilon} v) \gamma(\grave{\alpha} \rho)$

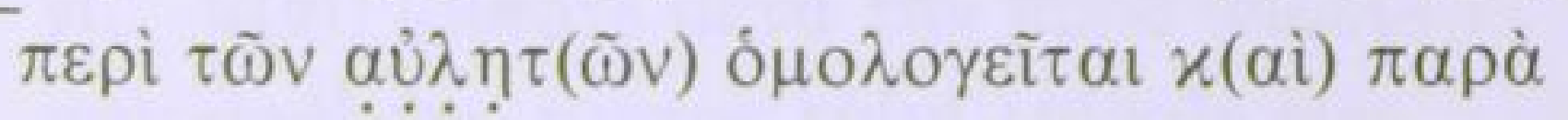




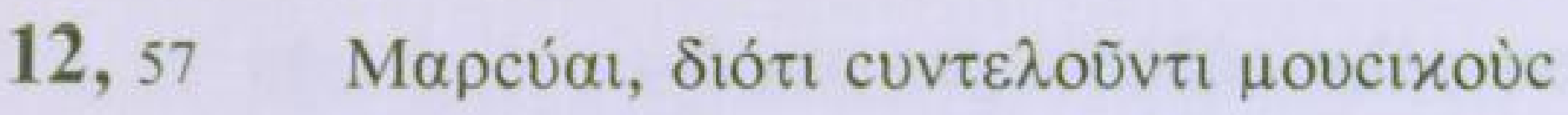

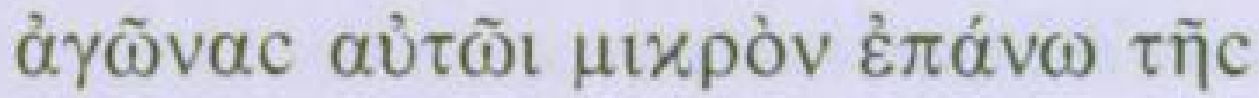

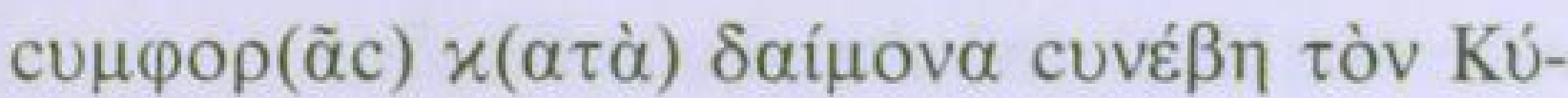

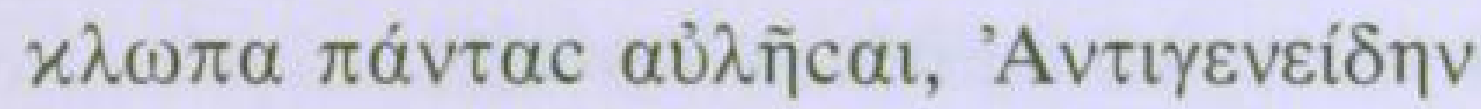

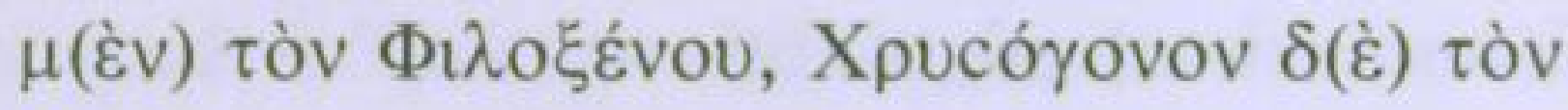

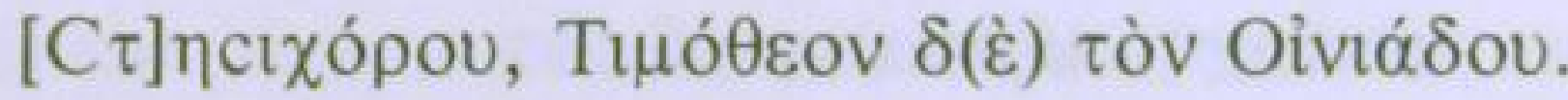

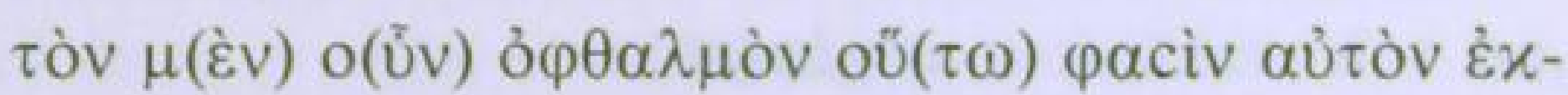

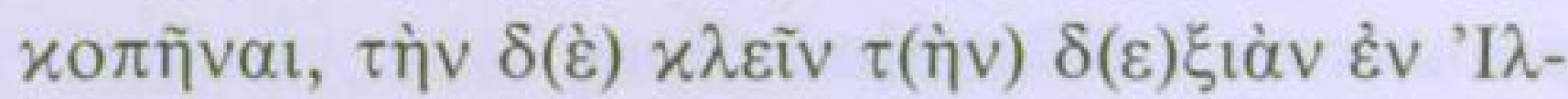

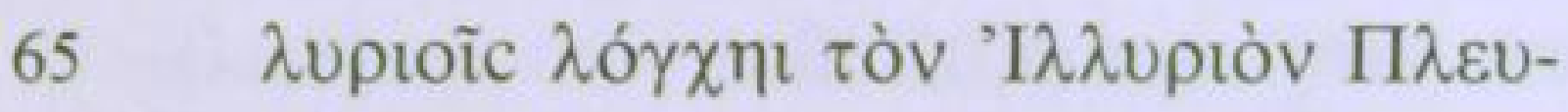

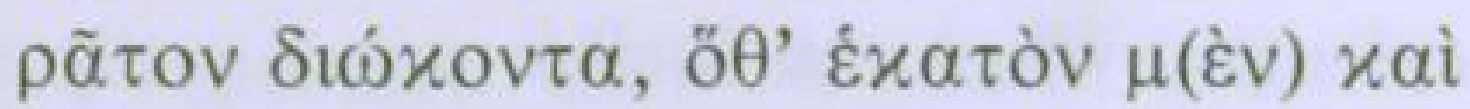

Col. 13

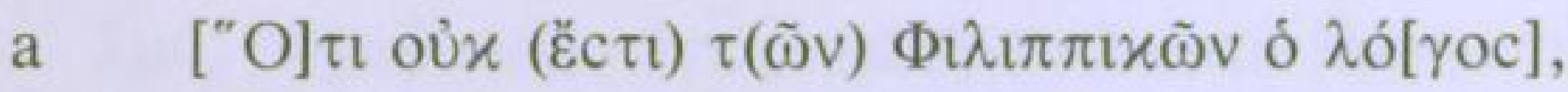

b $\quad \Delta \eta \mu \mathrm{oc} \theta \dot{\varepsilon}$ vouc $\delta(\grave{\varepsilon}) \alpha \check{\alpha} \lambda \lambda \omega \mathrm{c}$.

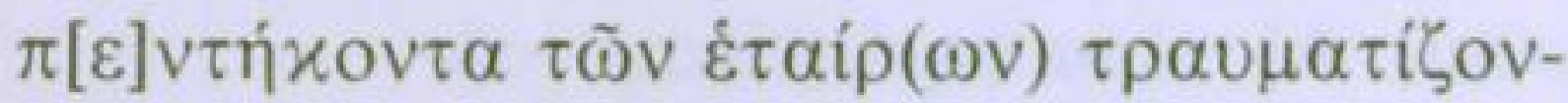

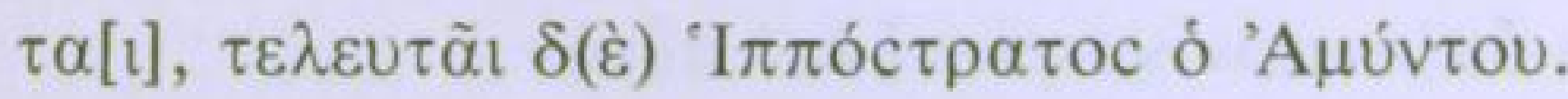
$\tau[\rho] i ́ \tau o v ~ \tau \rho \alpha \tilde{\mu} \mu \alpha \lambda[\alpha] \mu \beta a ́ v \varepsilon 1$ $\chi \alpha \tau \dot{\alpha} \tau \dot{\eta} v$

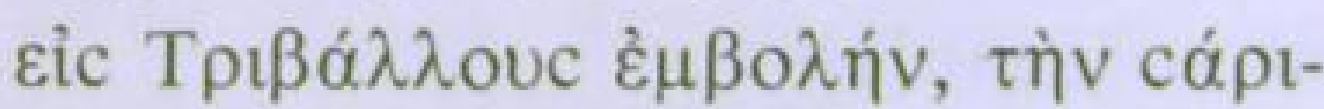




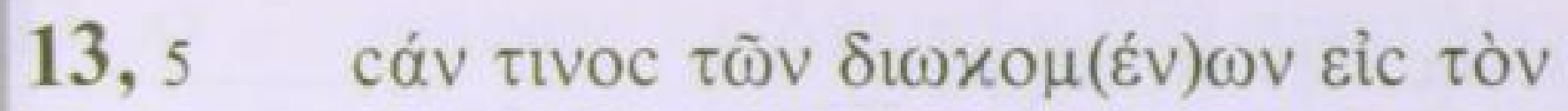

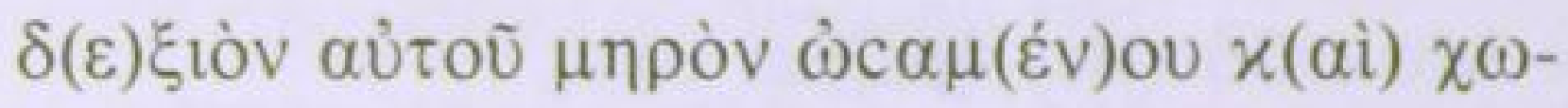

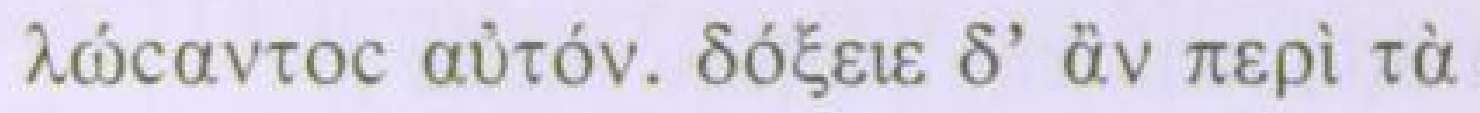

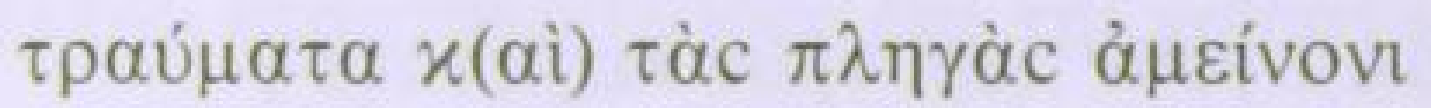

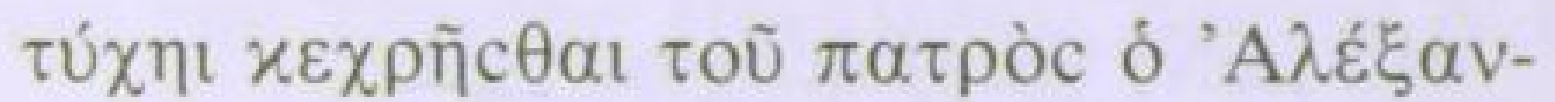

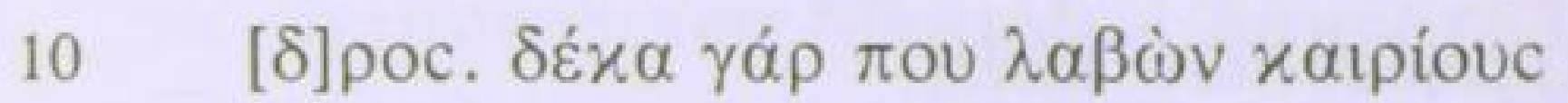

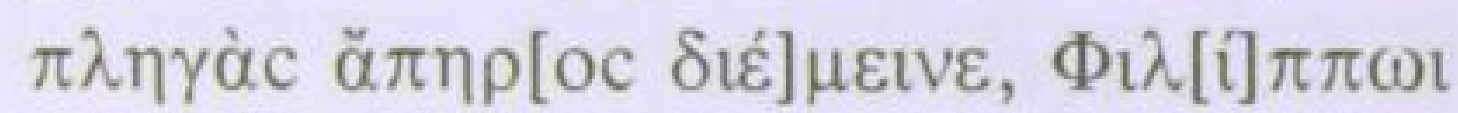

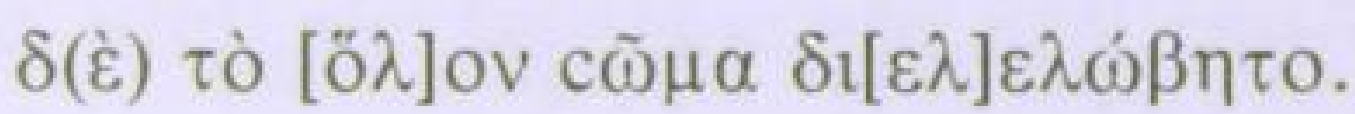

\section{$\overline{\mathrm{IB}}$}

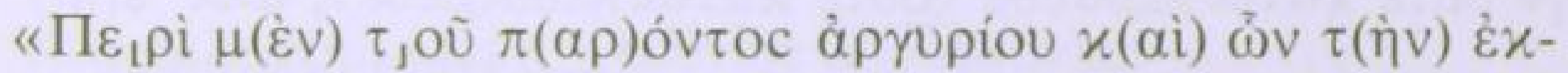

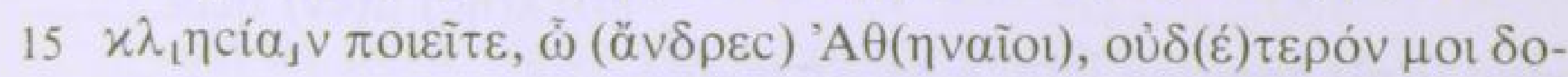

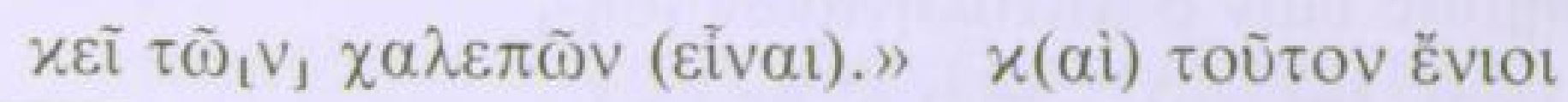

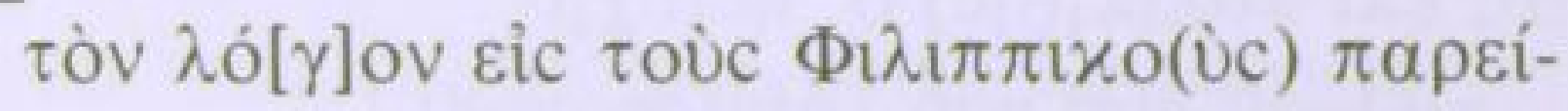

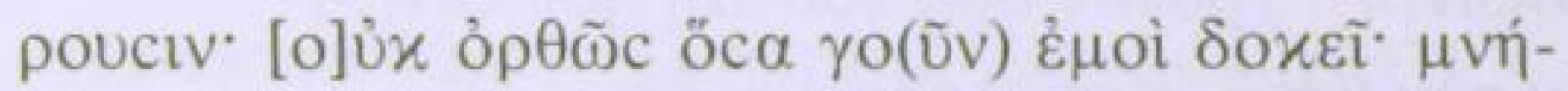

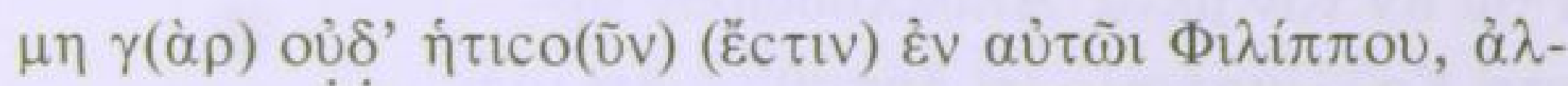

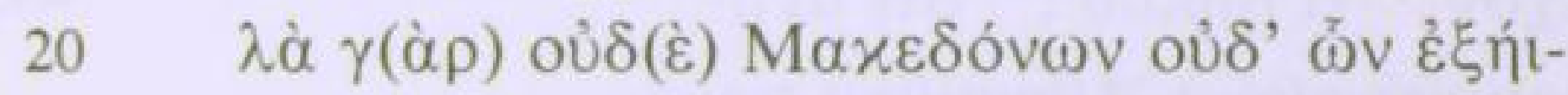

$[\rho] \eta x \varepsilon[\pi] \alpha \rho \dot{\alpha} \tau \dot{\alpha c} \operatorname{cuv} \theta \dot{\eta} x(\alpha c) x(\alpha i) \tau o u ̀ c ~ o ̈ \rho x o(v c)$

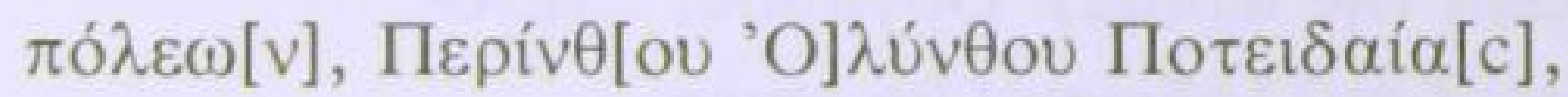

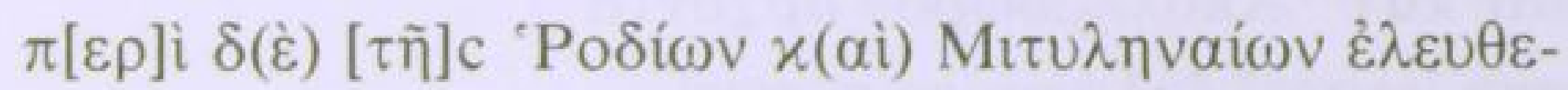

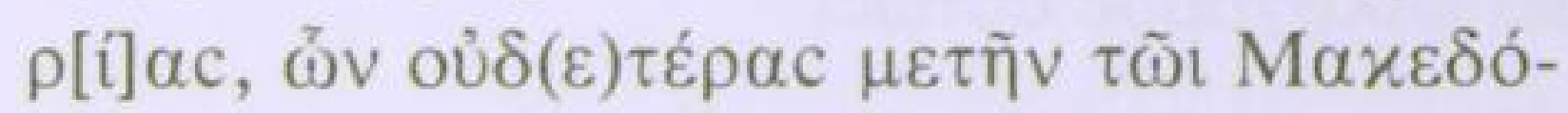

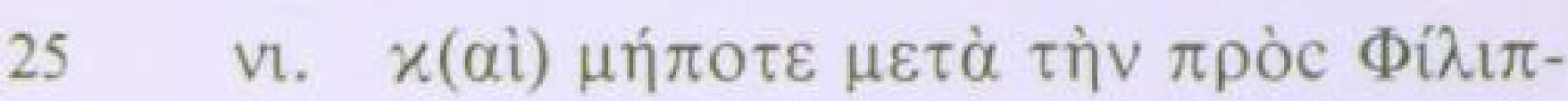

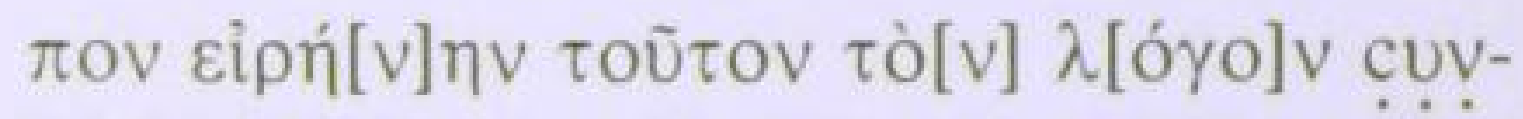




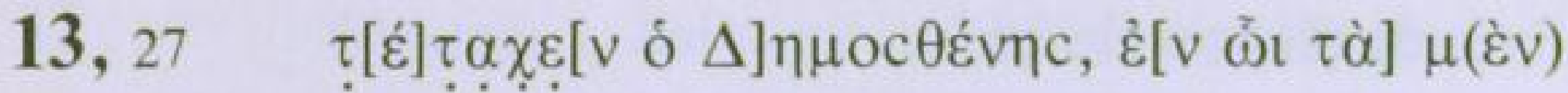

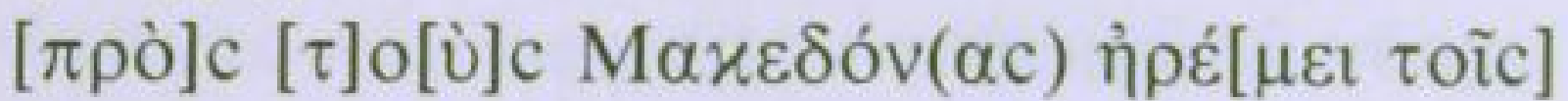

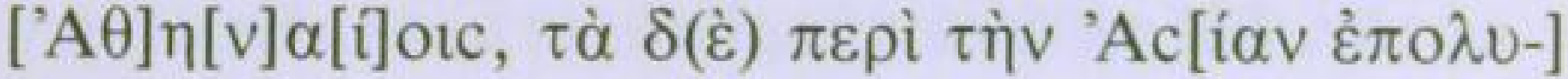

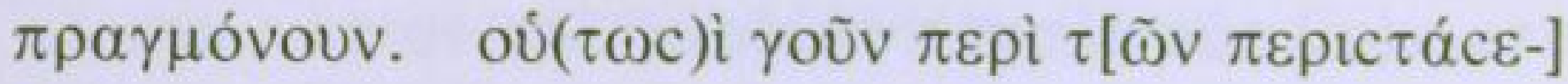

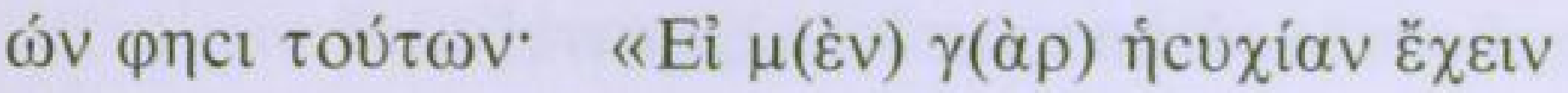

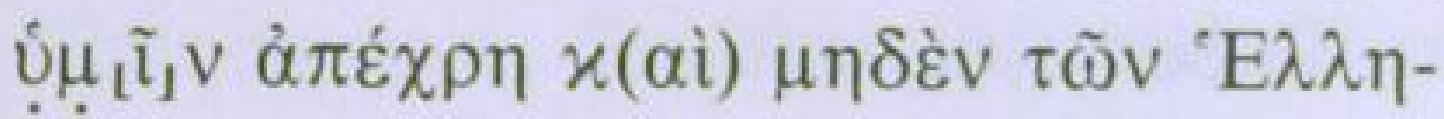

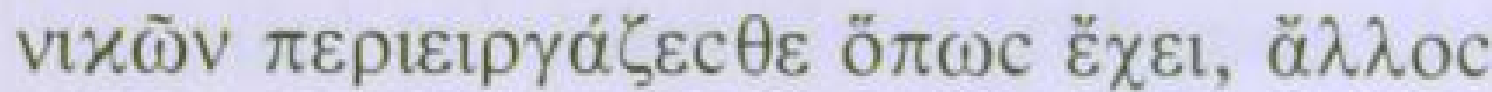

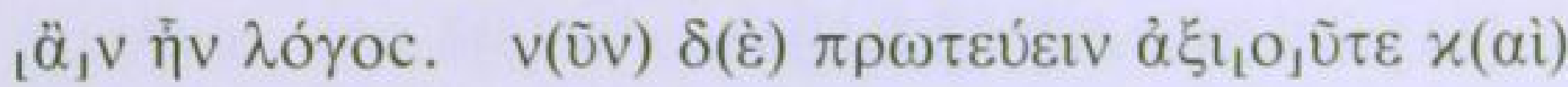

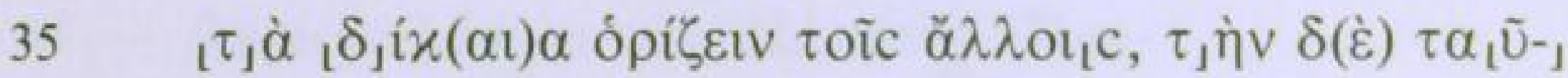
$\tau^{\prime} \dot{\varepsilon} \varphi o_{1} \delta \varepsilon v_{\jmath}, o u c \alpha v x(\alpha i) \varphi v \lambda \alpha \xi_{0}, v_{1} c \alpha v \delta v ́ v \alpha-$

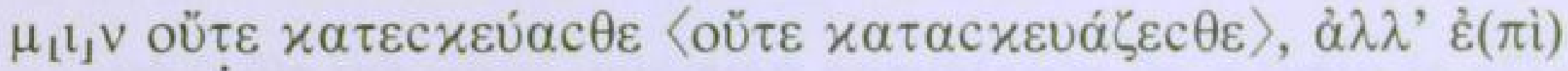
$\pi \mathrm{o} \lambda \lambda(\tilde{\eta} \mathrm{c}) \mu(\grave{\varepsilon} v) \dot{\eta} \mathrm{cu}-$

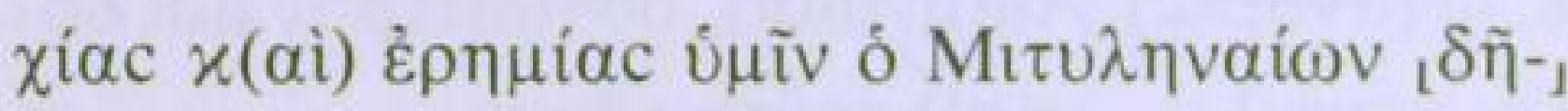

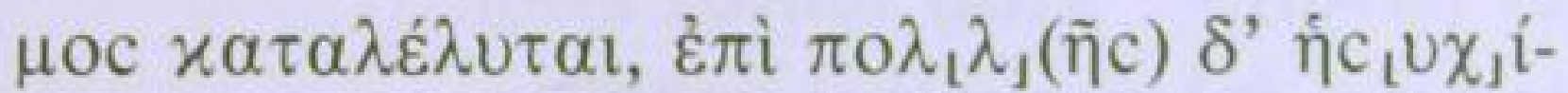

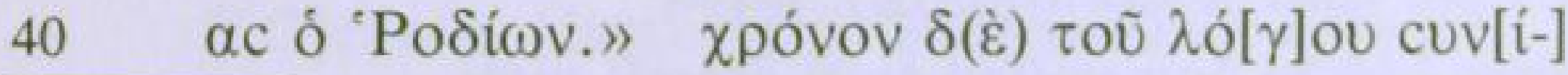

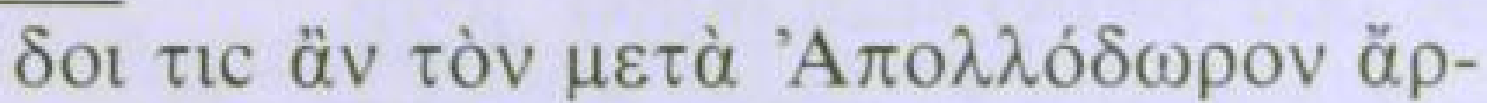

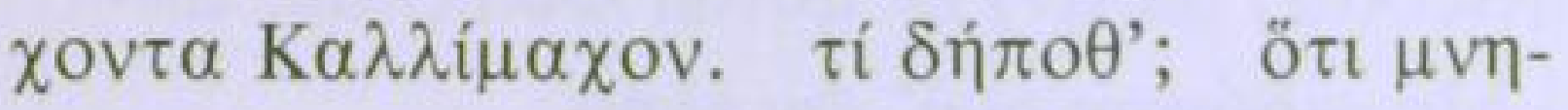

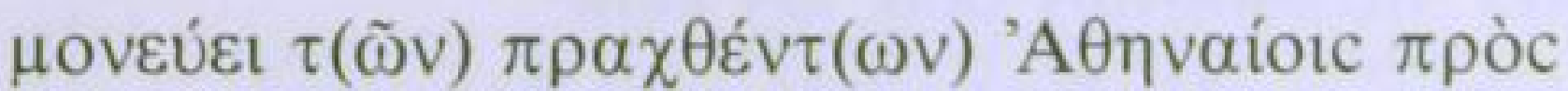

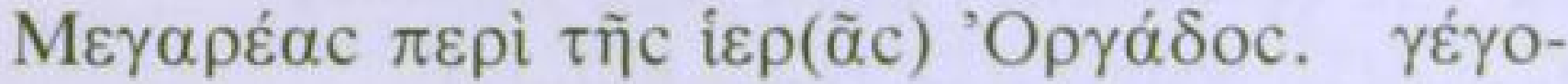

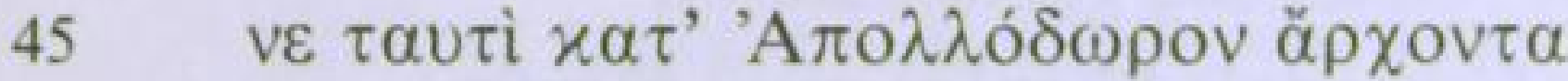

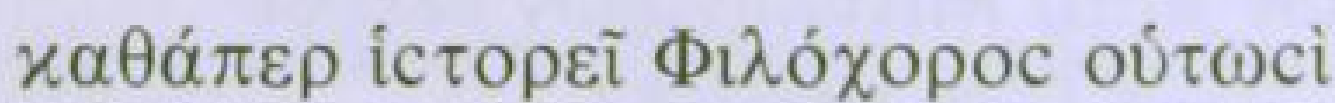




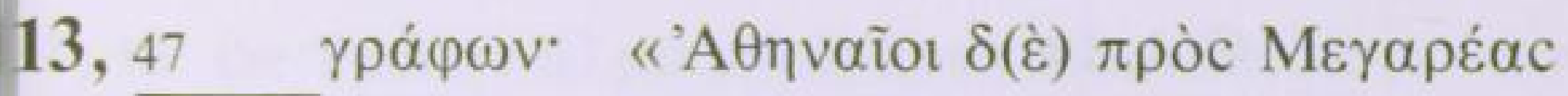

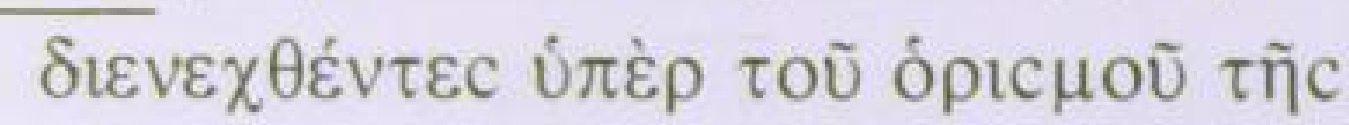

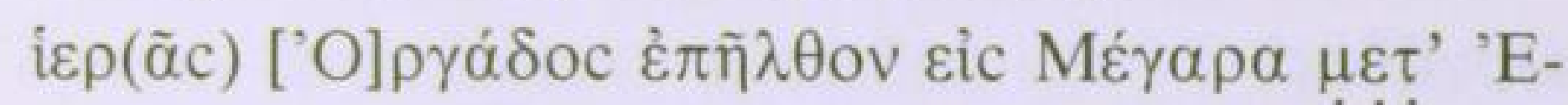

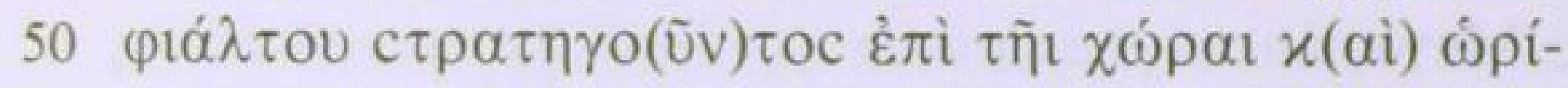

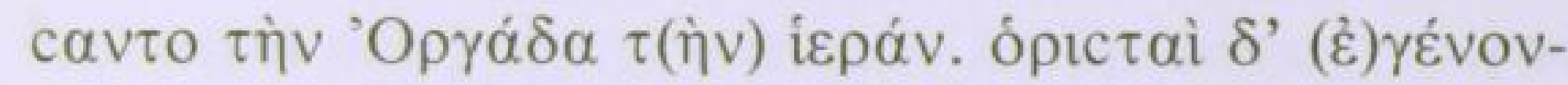

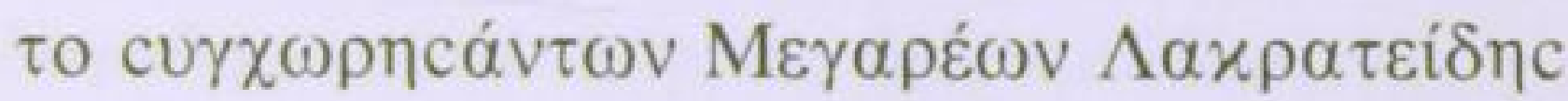

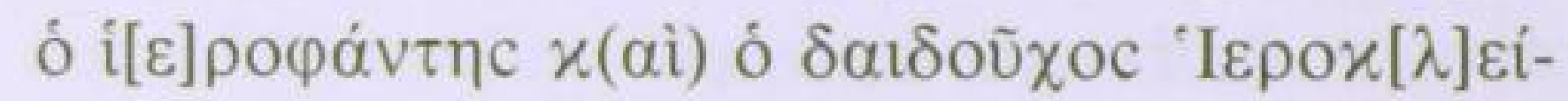

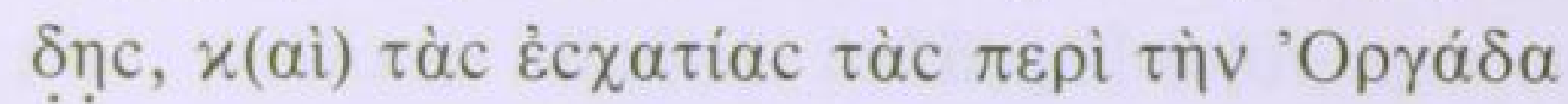

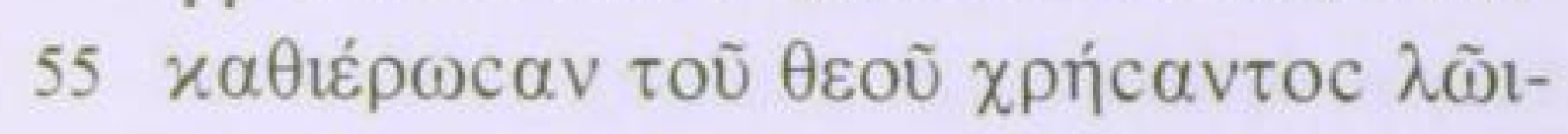

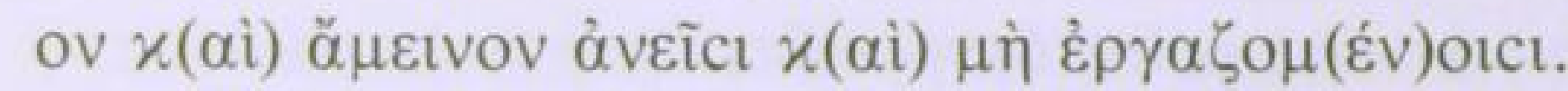

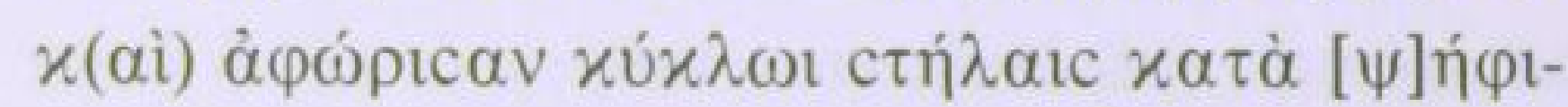

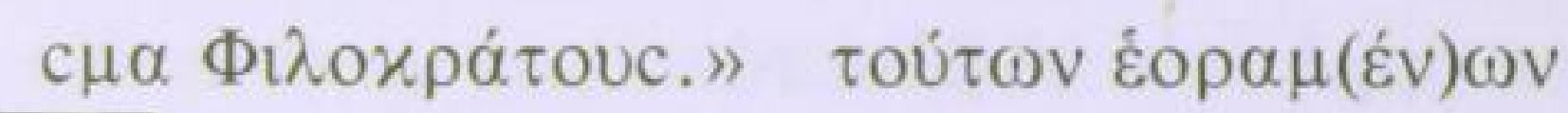

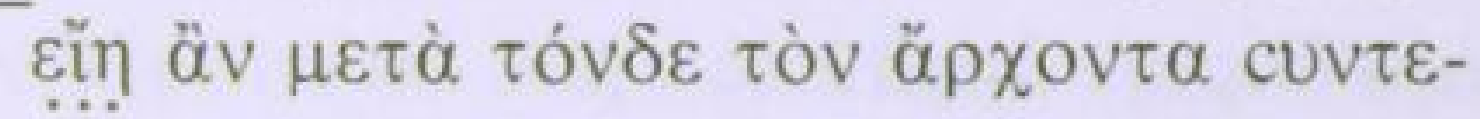

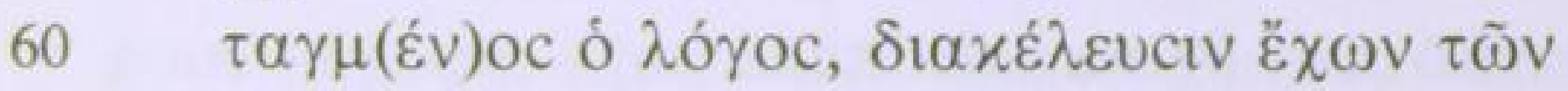

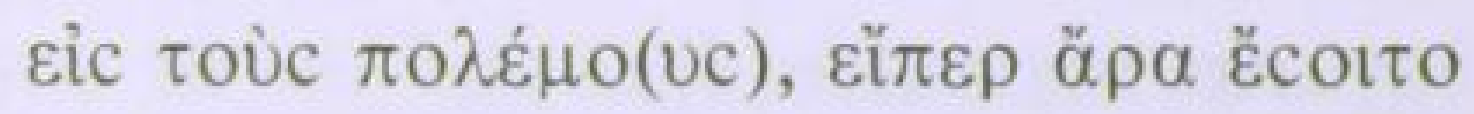

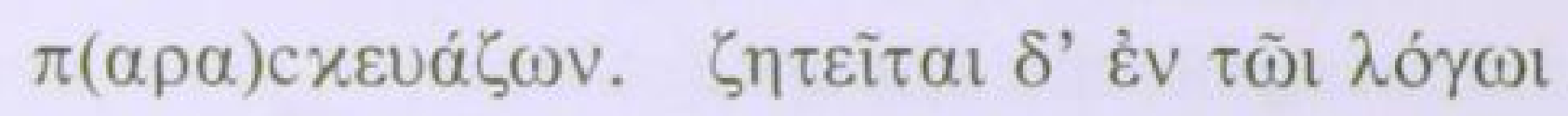


a $\Pi(\varepsilon \rho i) \tau \tilde{\eta} c^{\prime} O \rho \gamma \alpha ́ \delta$ oc.

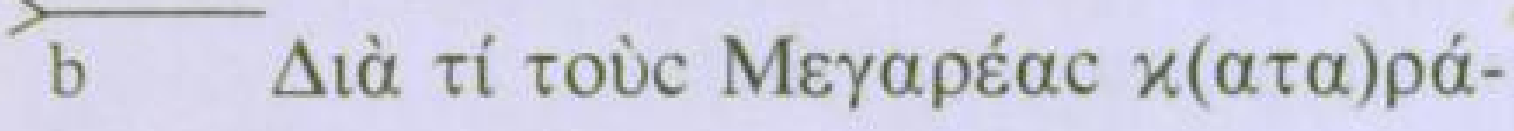

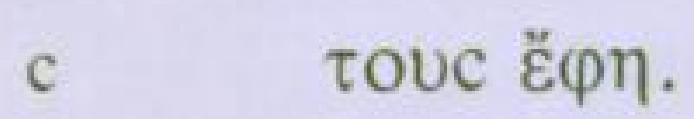

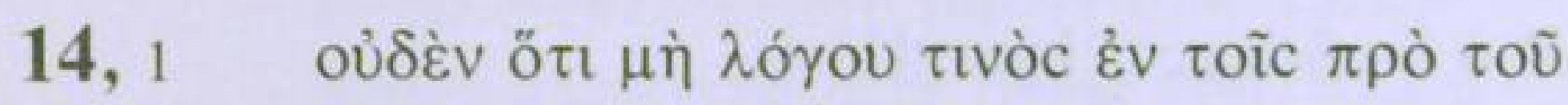

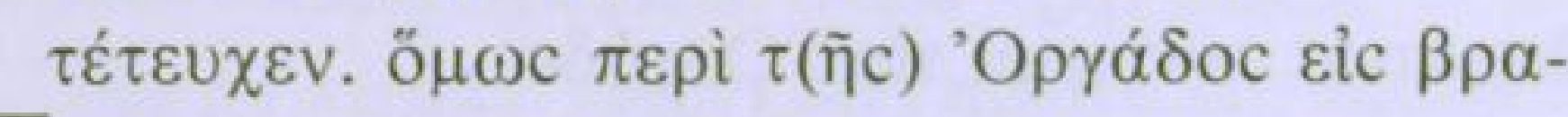

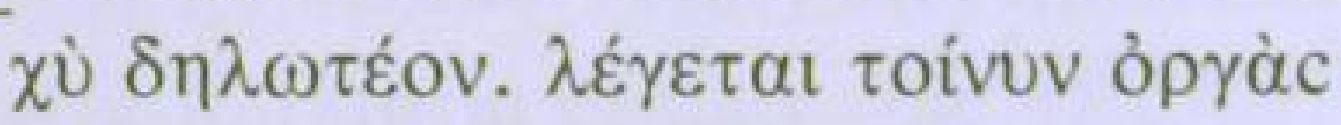

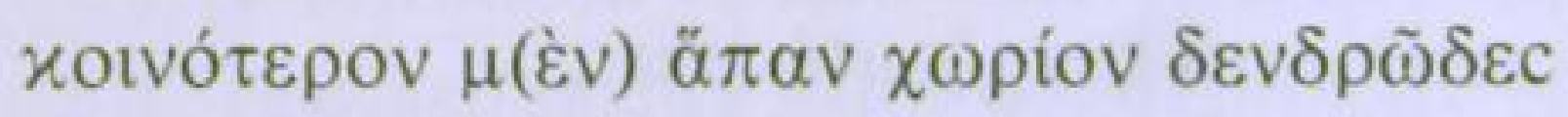

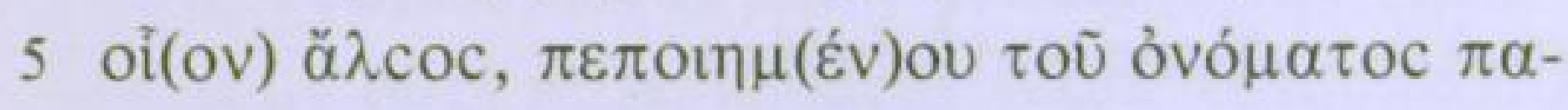

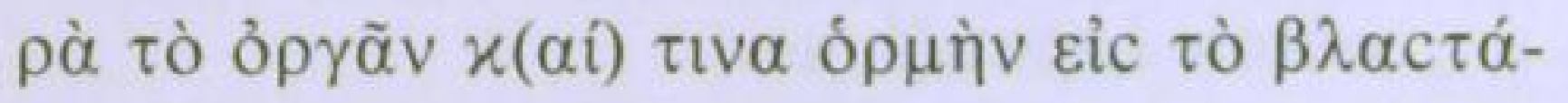

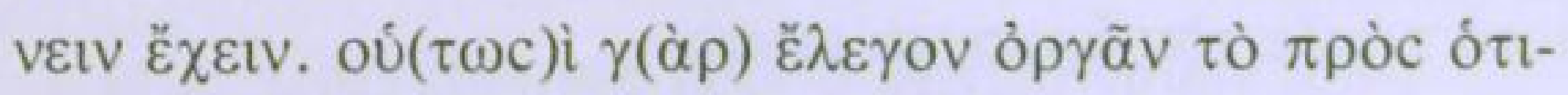

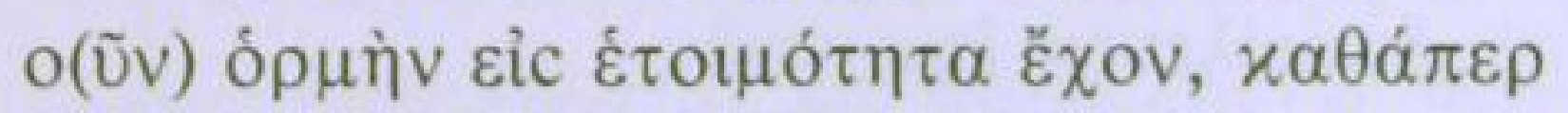

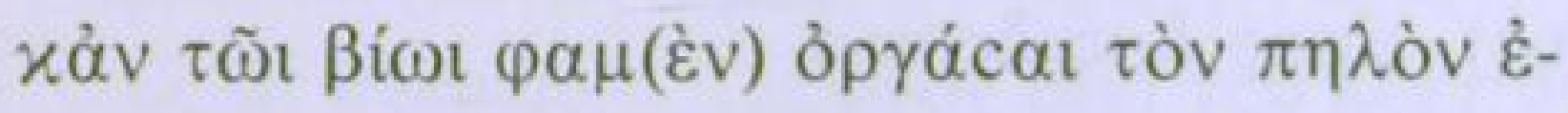

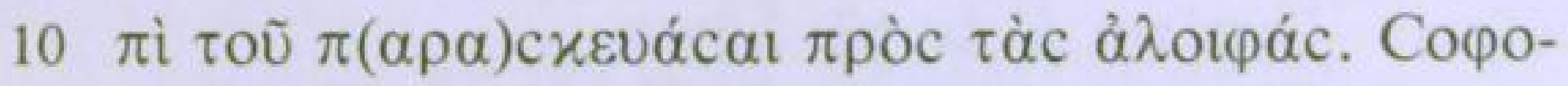

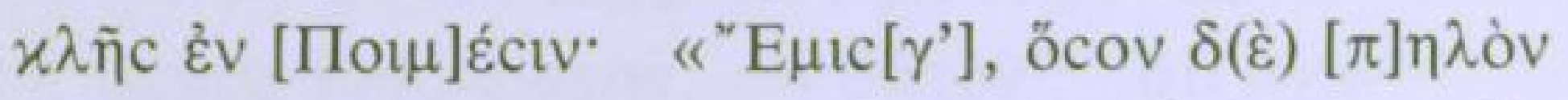

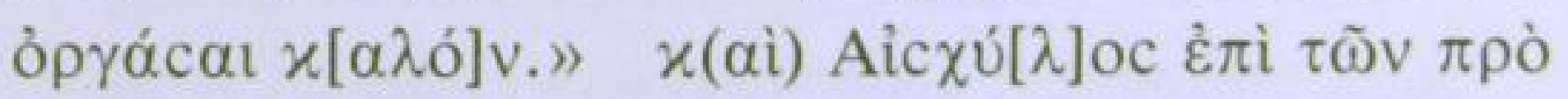

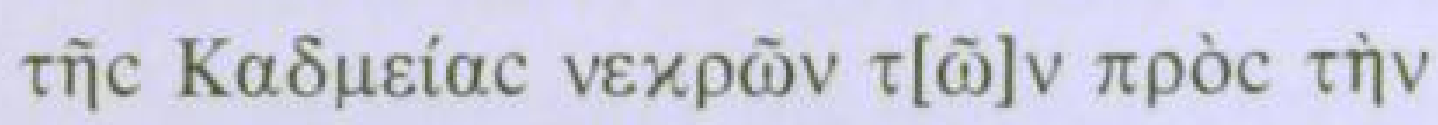

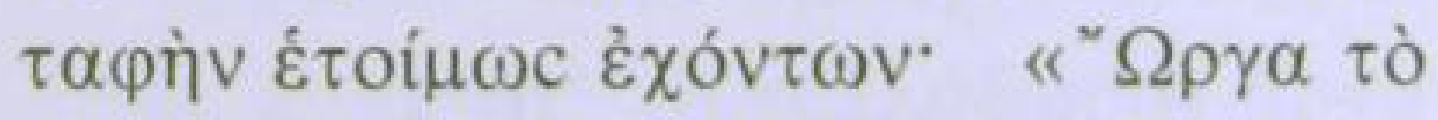

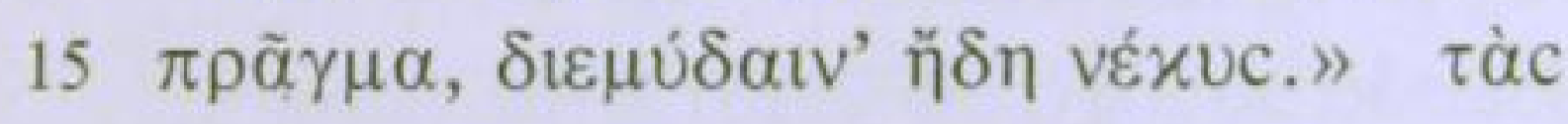

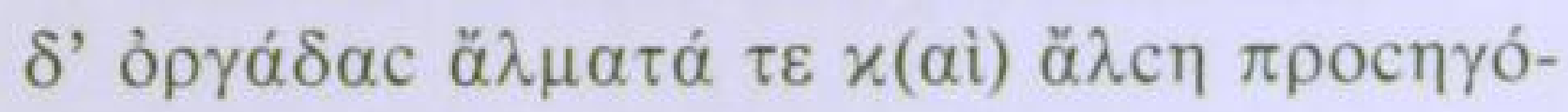

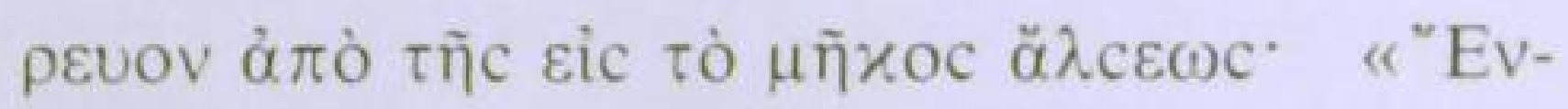




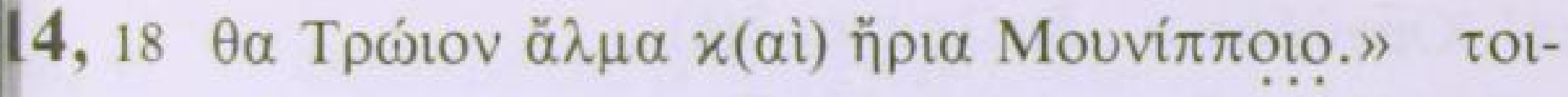

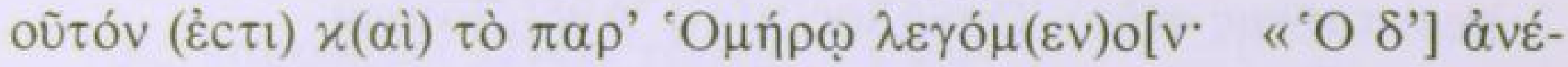

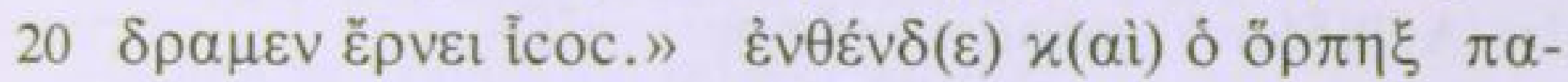

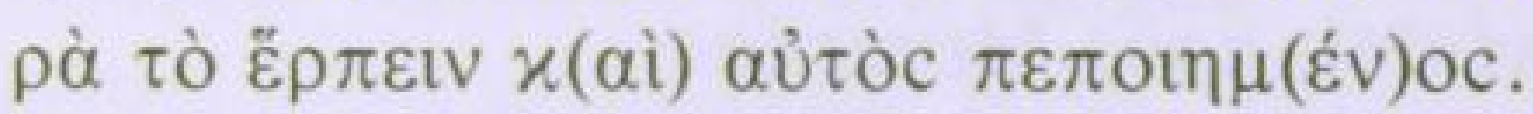

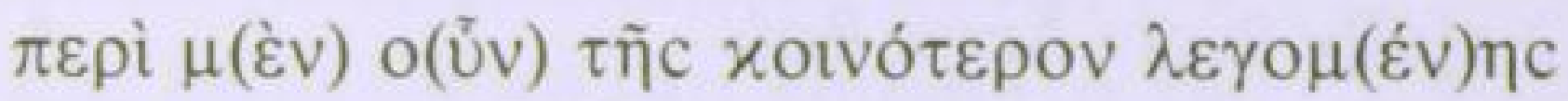

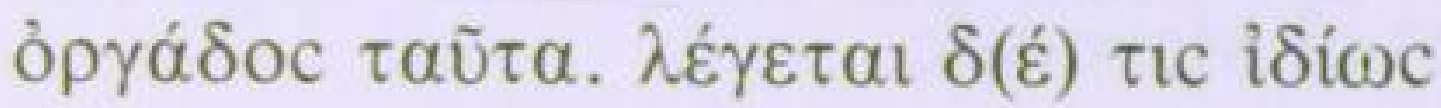
$\pi \alpha \rho \alpha \grave{~ M \varepsilon \gamma \alpha \rho \varepsilon v ̃ c ı v ~ ' O \rho \gamma \alpha ̀ c ~ o ̉ v o \mu \alpha c \tau l-~}$

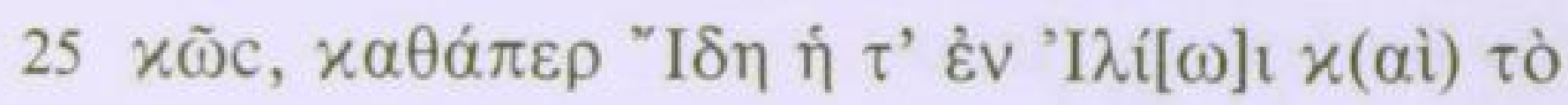

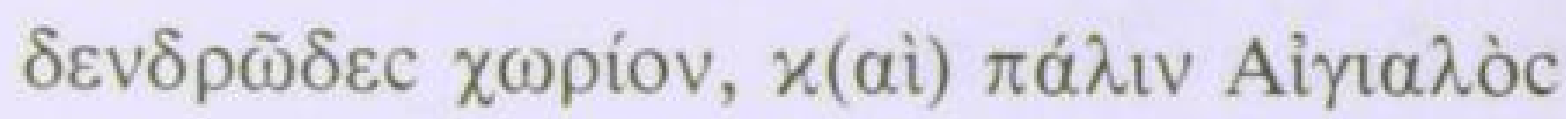

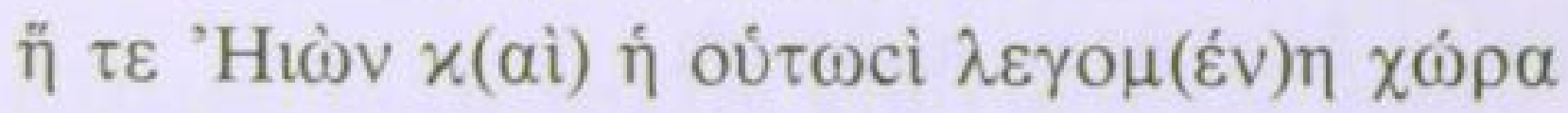

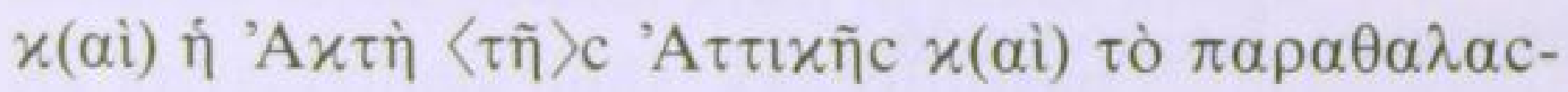

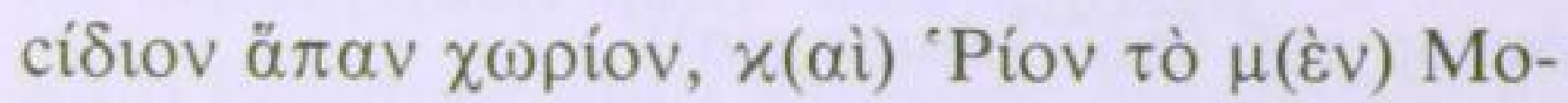

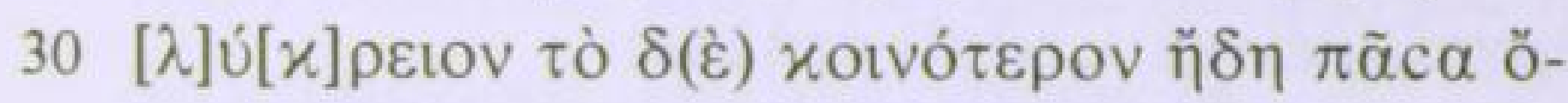

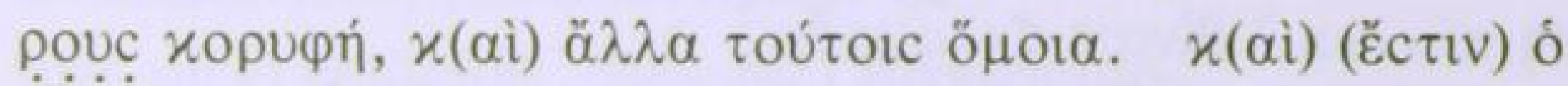

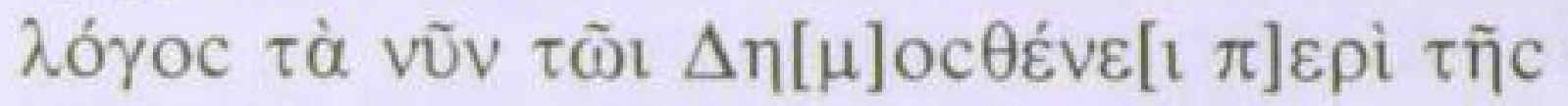

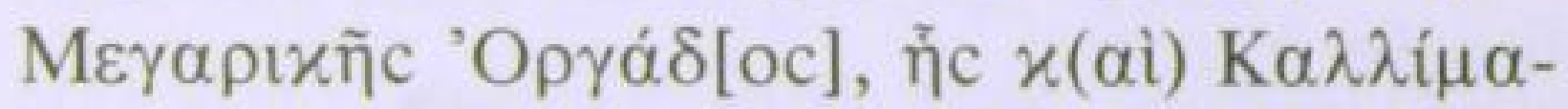

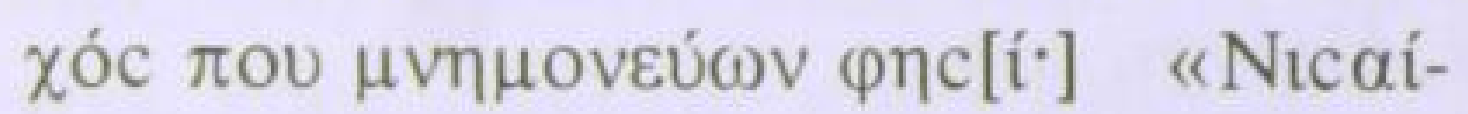




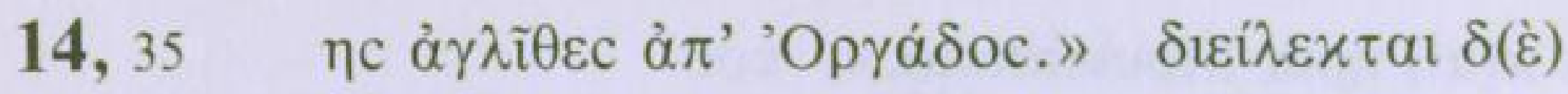

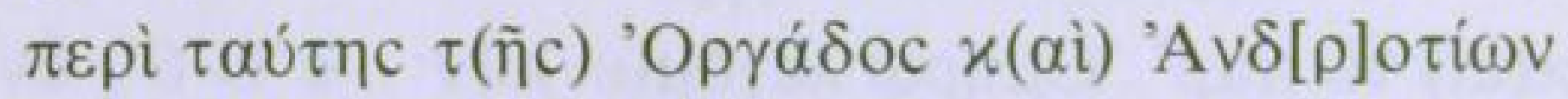

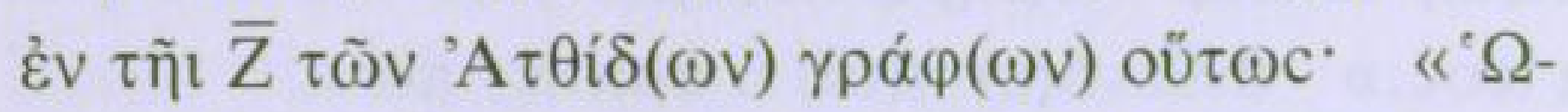

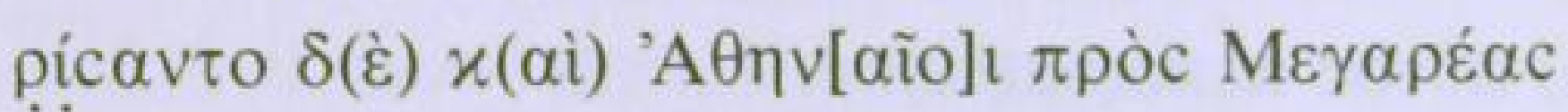

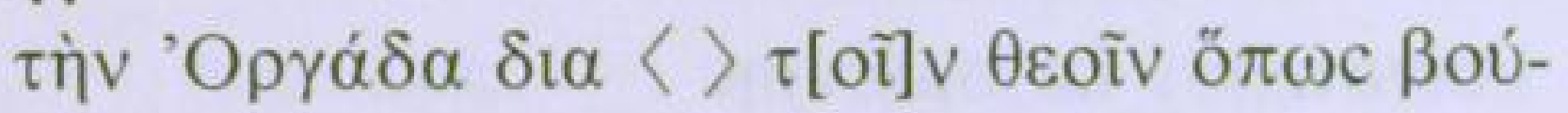

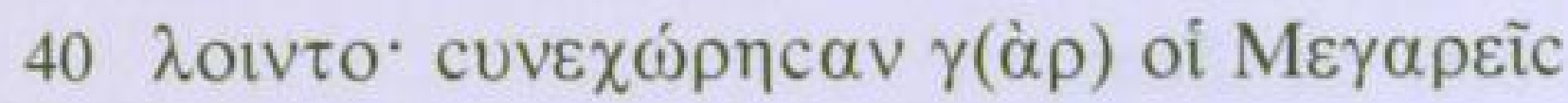

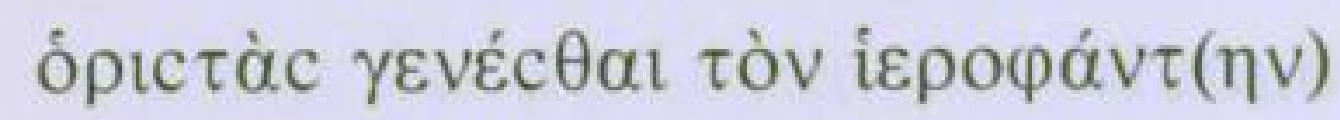

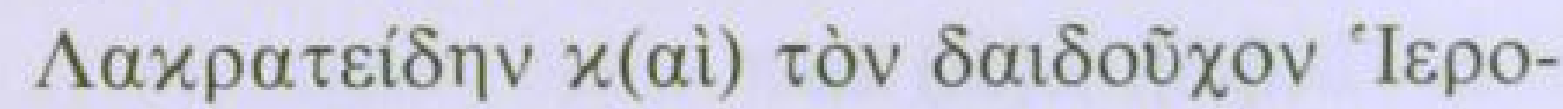

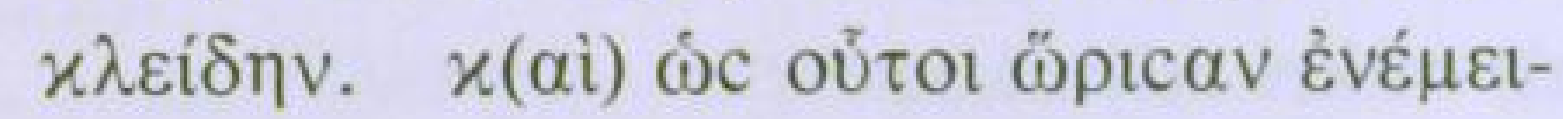

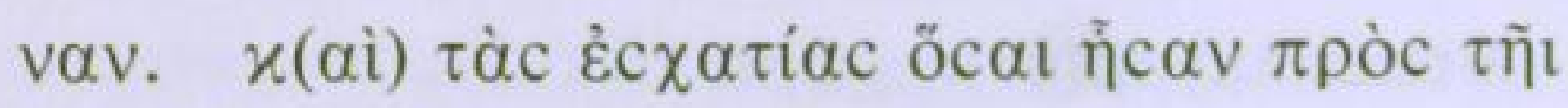

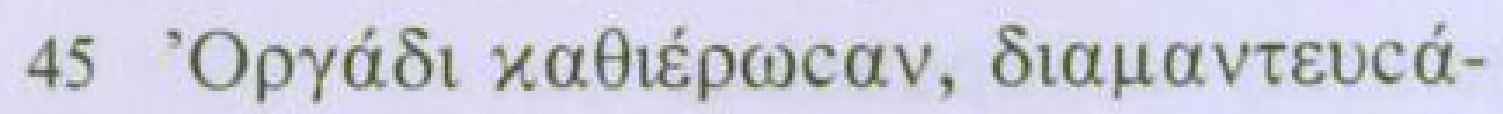

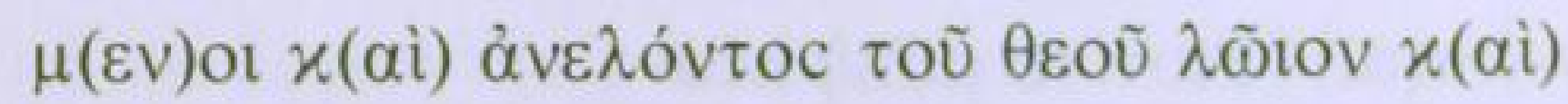

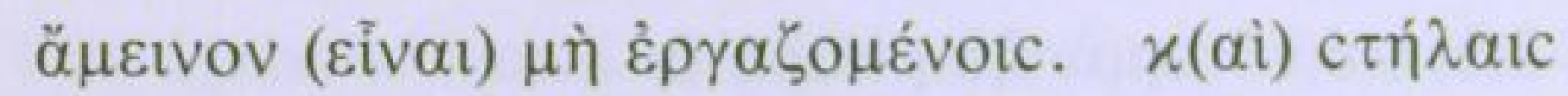

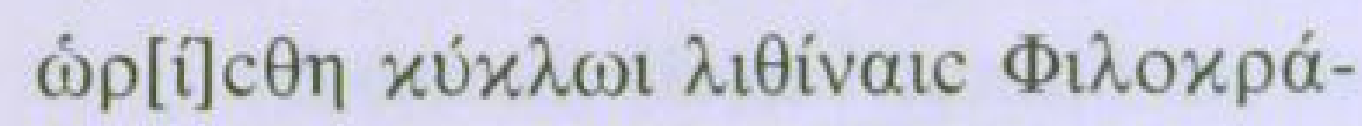

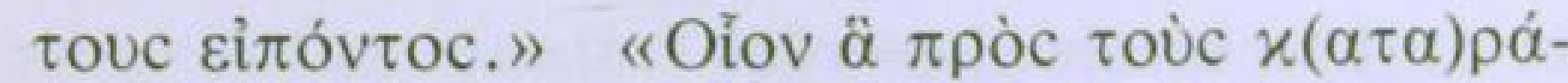

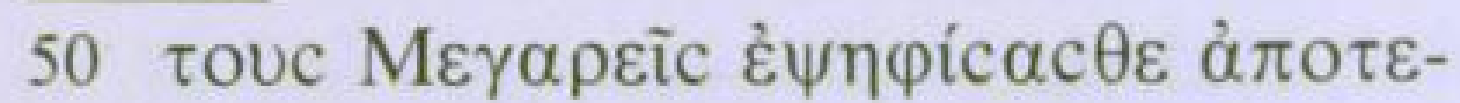

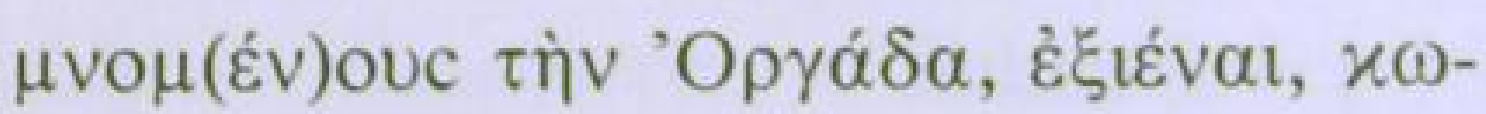

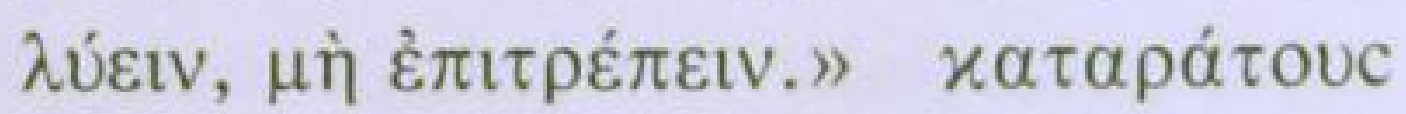

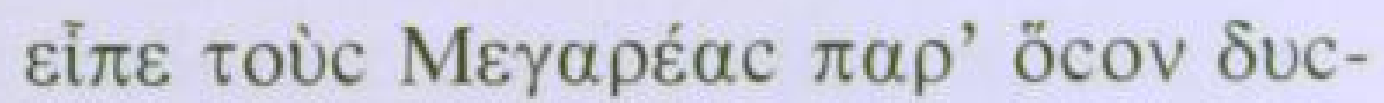

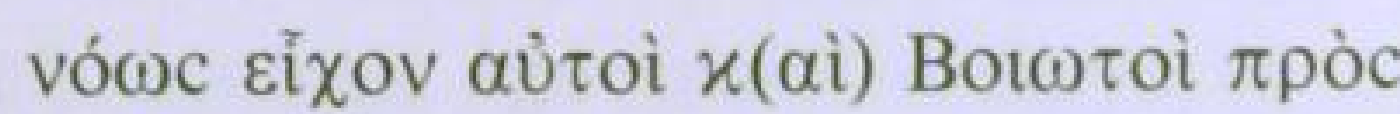

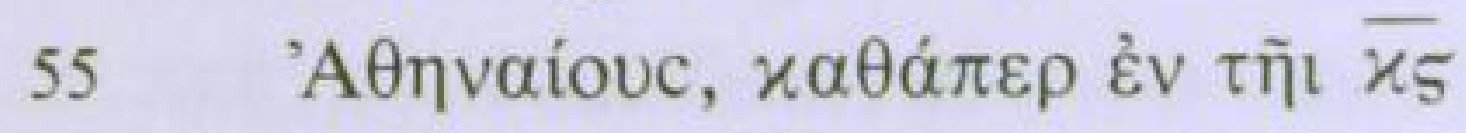

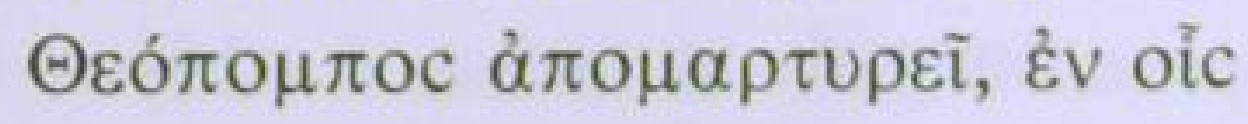




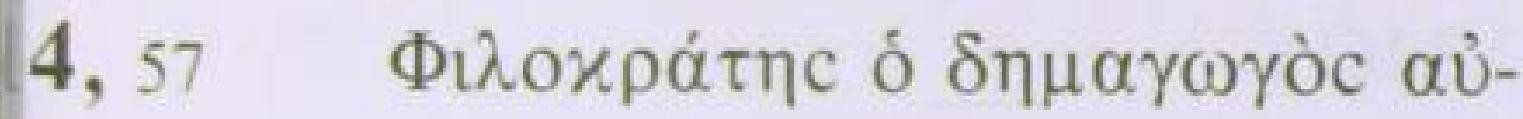

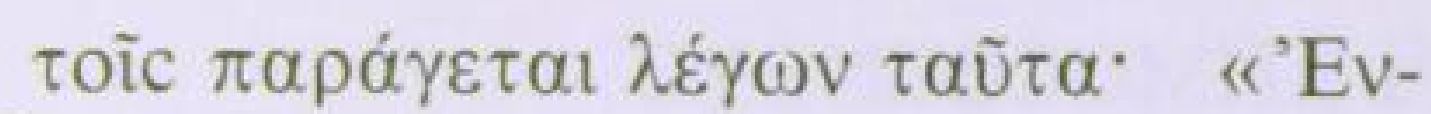

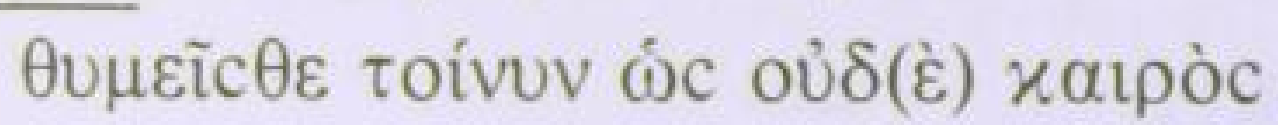

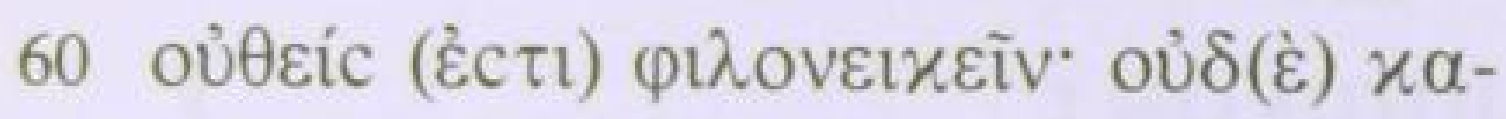

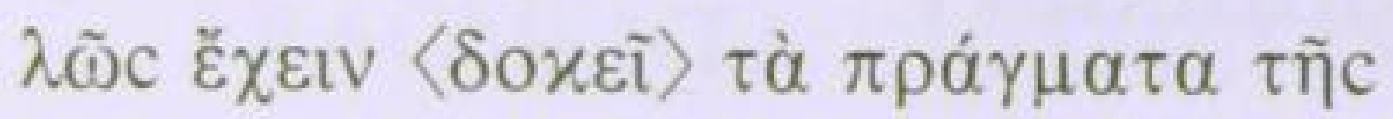

\section{Col. 15}

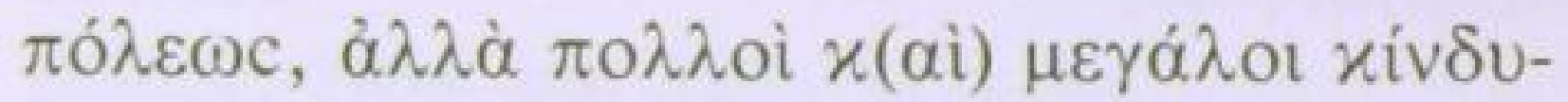
vol $\pi \varepsilon \rho \iota c[\tau] \tilde{\alpha} c \iota v \dot{\eta} \mu \tilde{\alpha} c \cdot \dot{\varepsilon} \pi \mathrm{\iota c} \tau \dot{\alpha}[\mu] \varepsilon \theta \alpha$

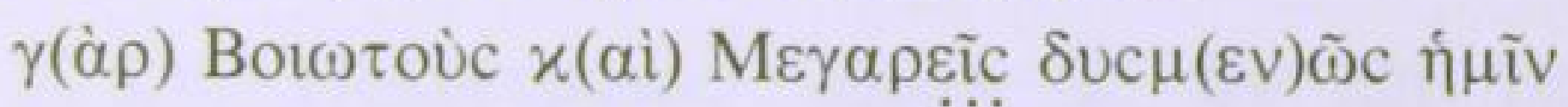

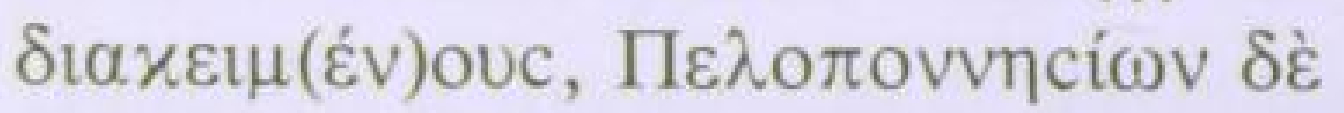

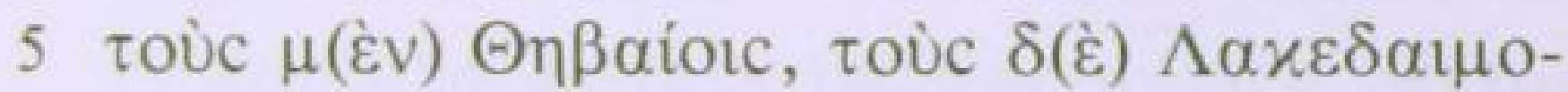

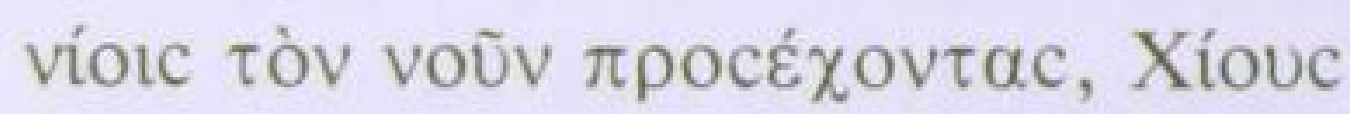

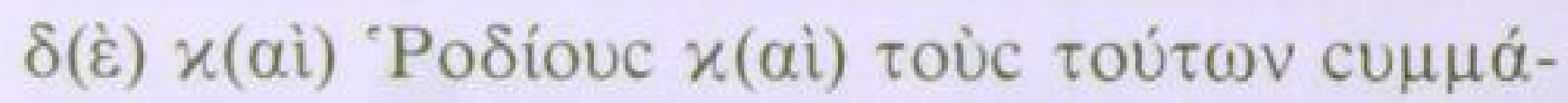

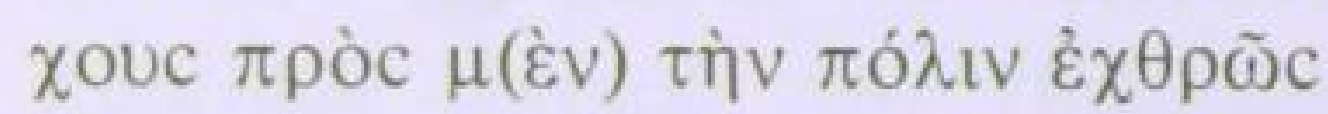

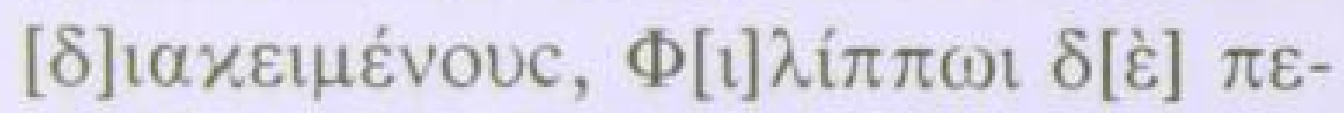

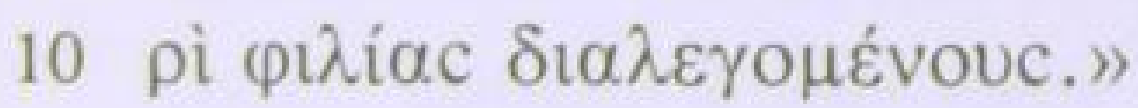




\section{$-\quad \Delta \mathrm{I} \Delta \mathrm{YMOY}$ \\ ПЕPI $\triangle \mathrm{HMOC \Theta ENOYC}$}

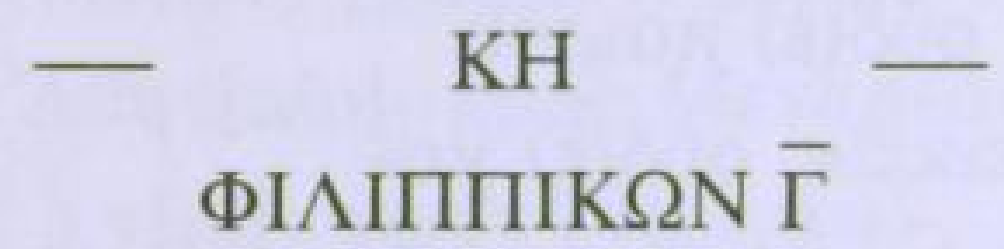

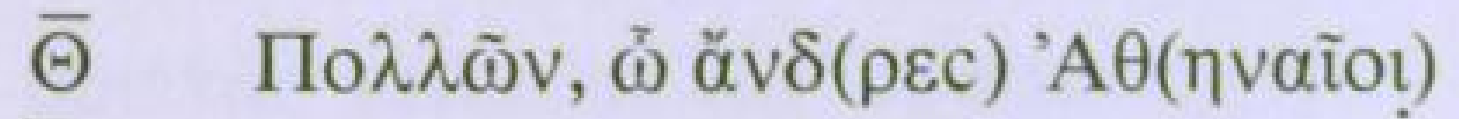

Dem. 9

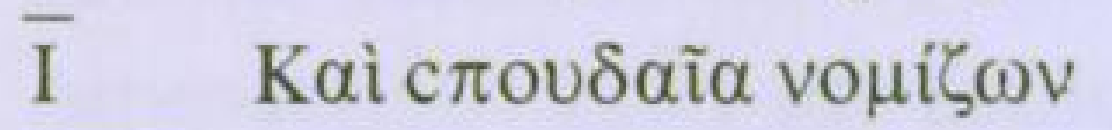

Dem. 10

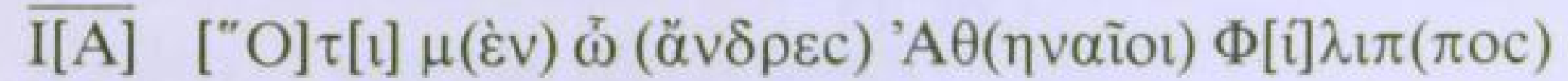

Dem. 11

$\overline{\mathrm{IB}} \quad \Pi \varepsilon \rho \grave{\mu} \mu \dot{\varepsilon}[v \tau] \circ \tilde{} \pi(\alpha \rho) \operatorname{v} v(\tau o c)$

Dem. 13 


\section{FRAGMENTA DIDYMEI IN DEMOSTHENEM COMMENTARII EX HARPOCRATIONE COLLECTA}

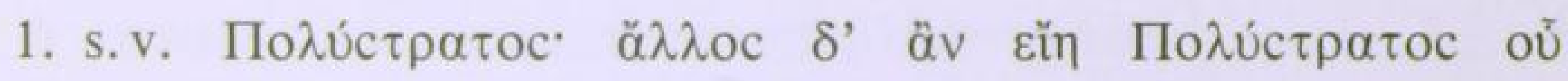

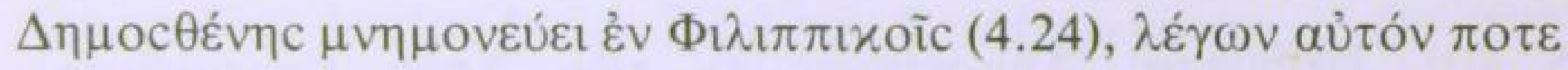

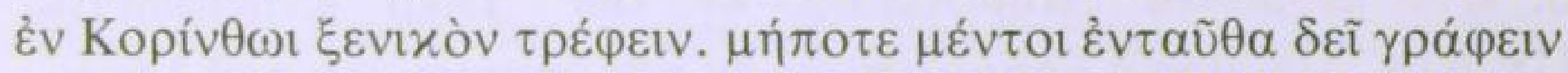

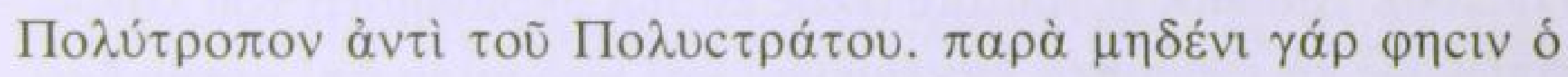

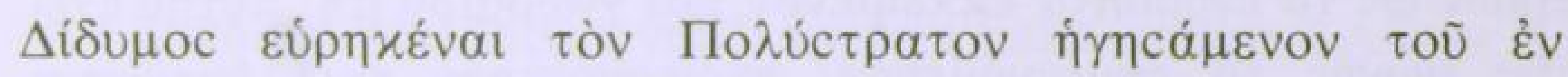

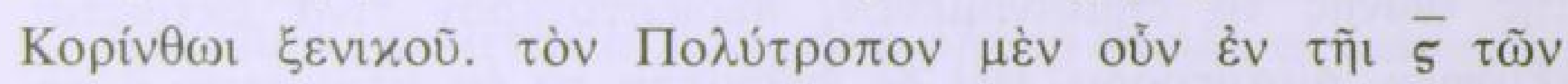

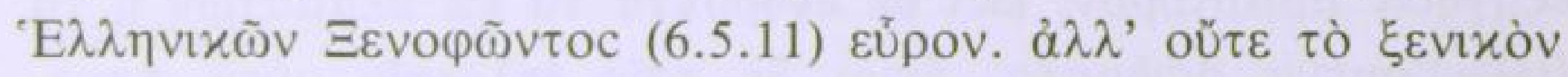

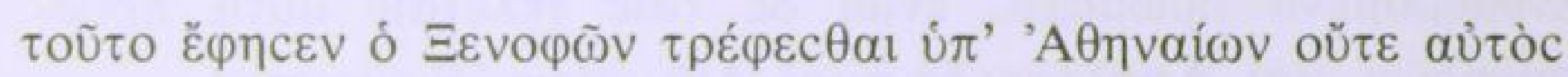

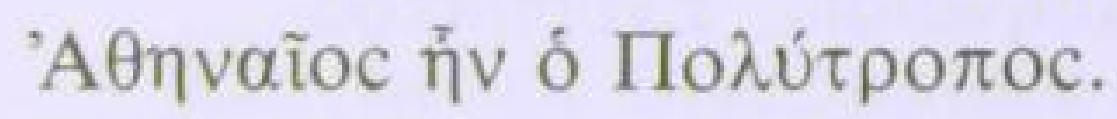

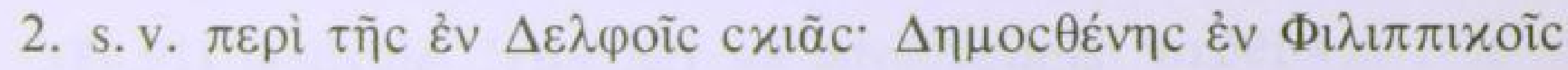

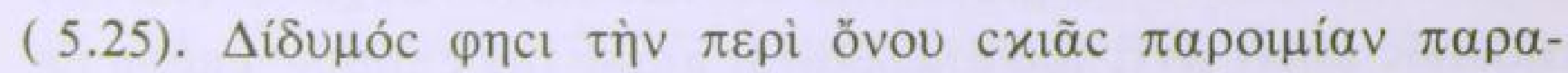

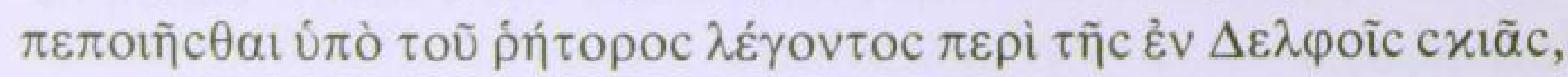

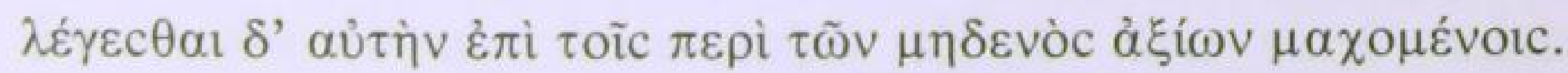

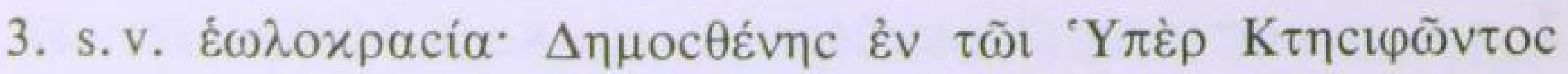

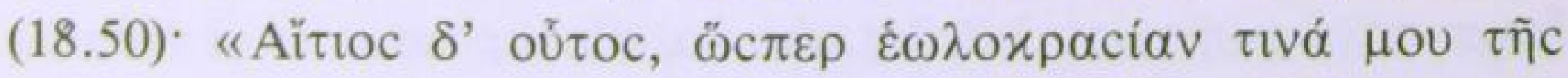

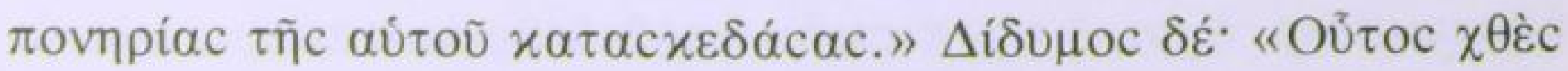




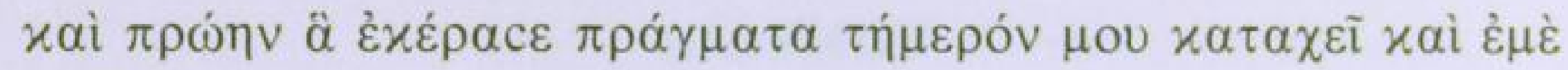

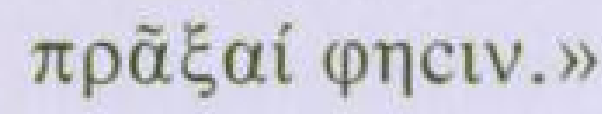

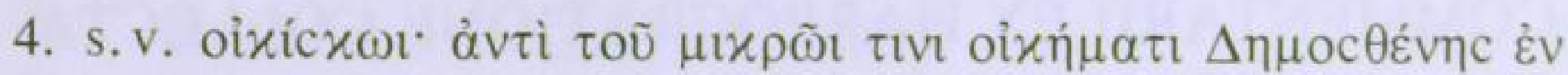

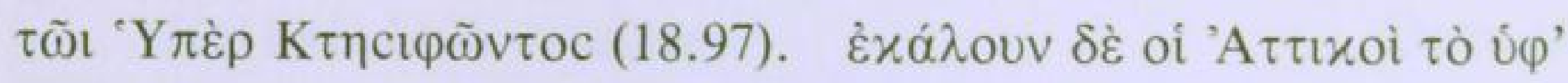

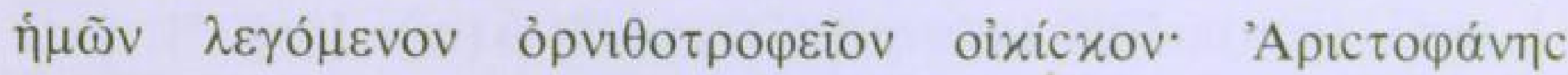

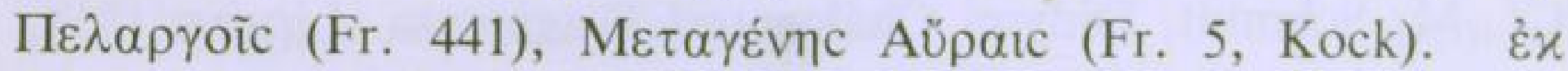

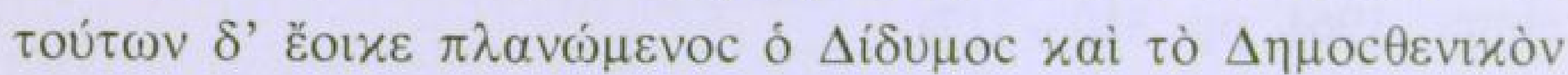
$\varepsilon \xi \xi \eta \gamma \varepsilon \tilde{\imath} c \theta \alpha$ l.

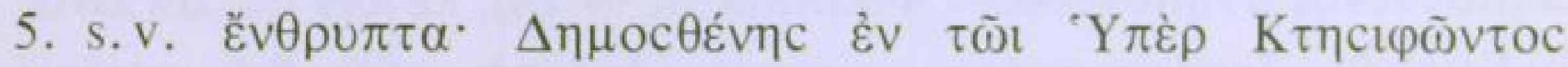

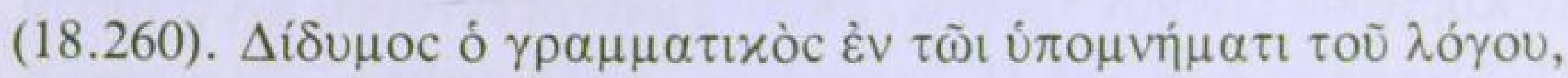

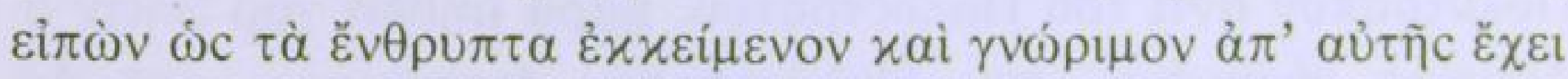

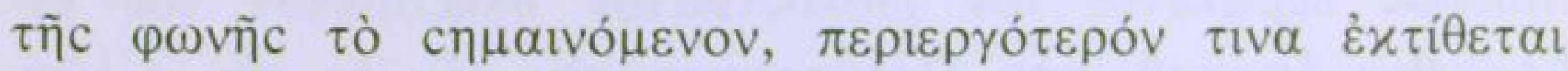

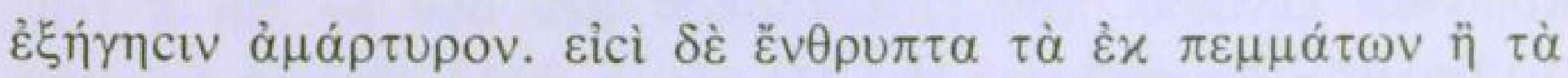

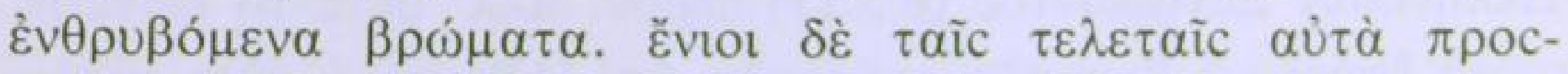

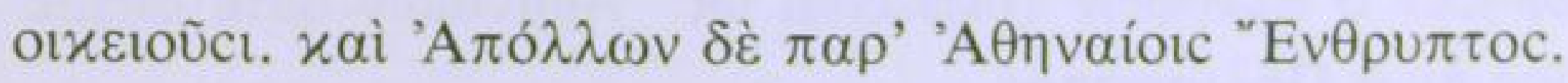

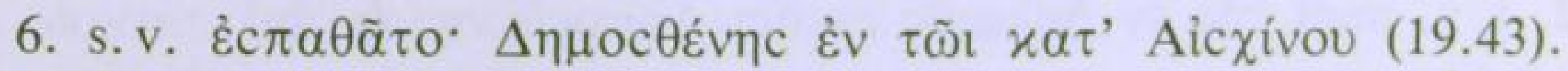

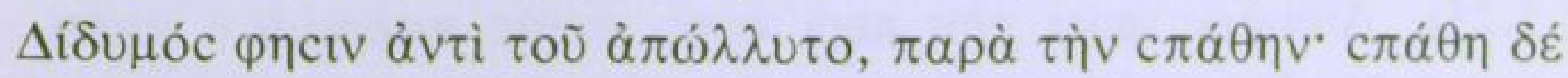

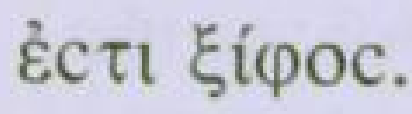

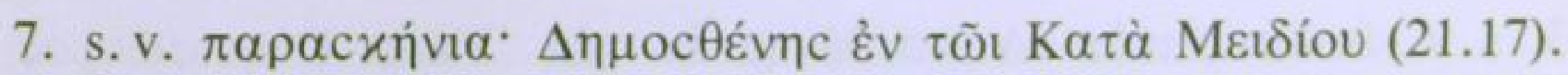

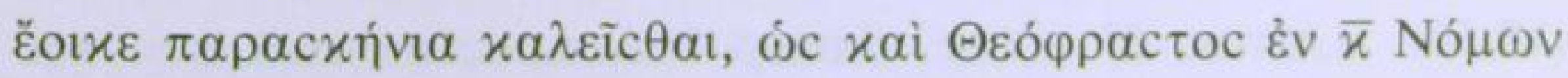

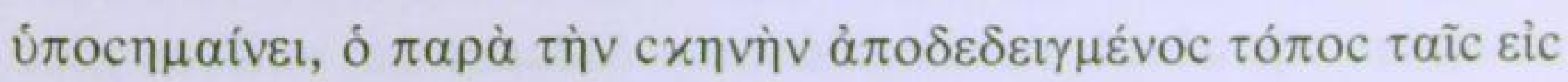

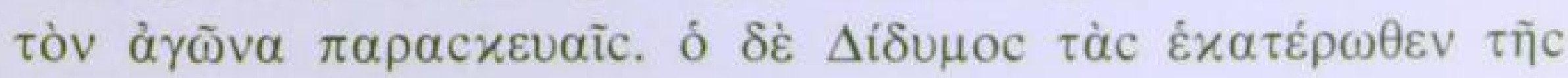

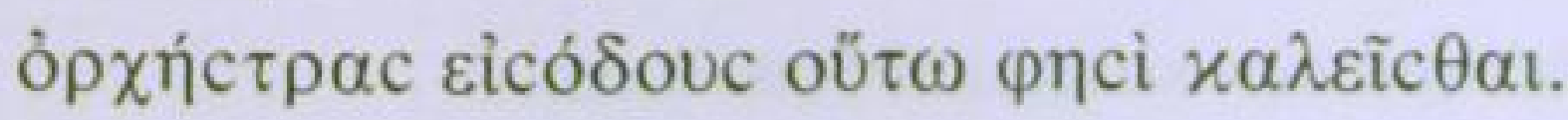




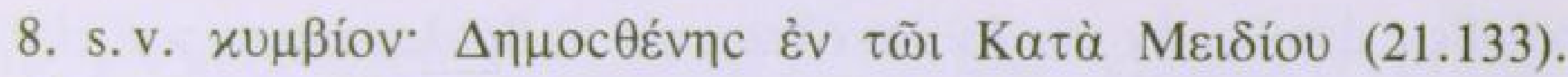

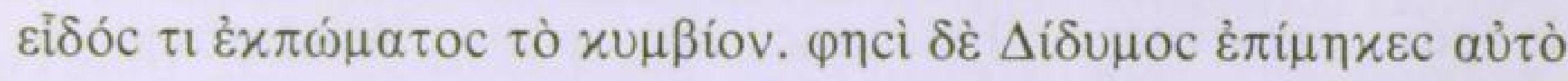

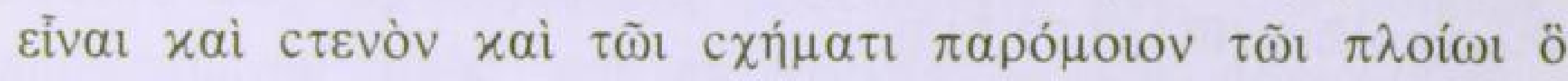
$\chi \alpha \lambda \varepsilon i \tau \alpha \imath$ $x u \beta$ B́ov.

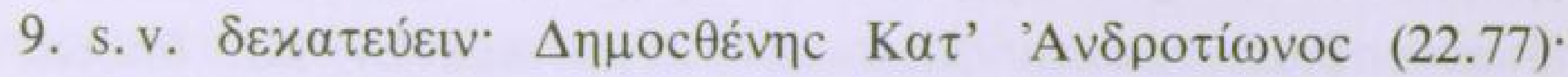

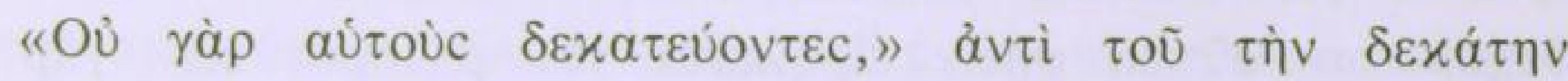

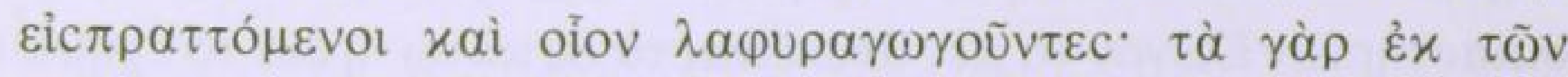

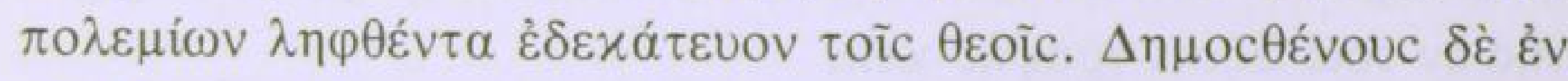

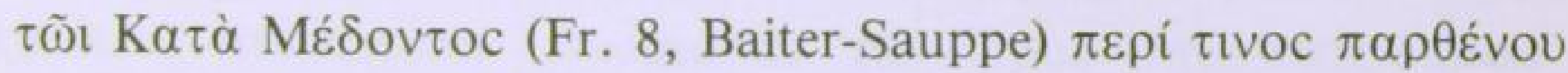

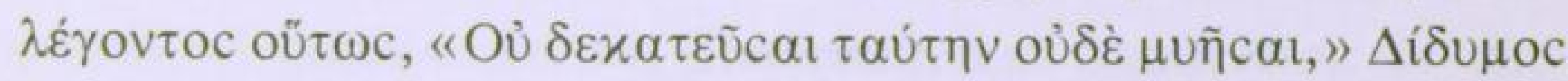

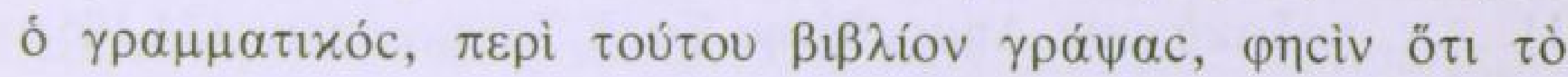

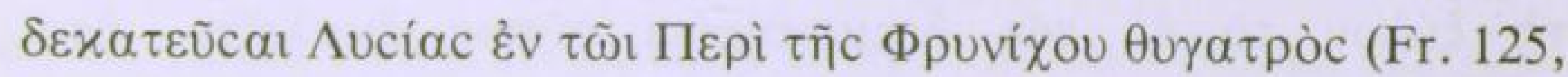

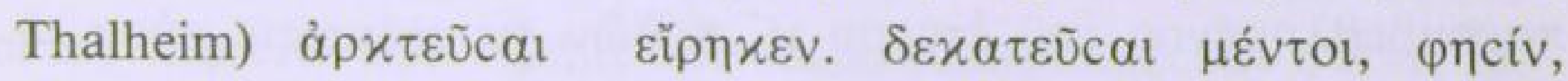

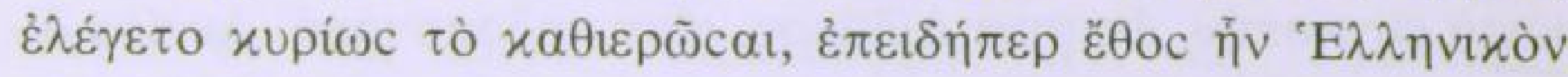

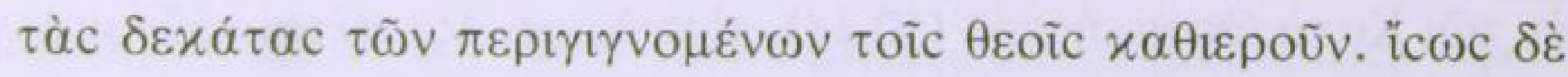
$\tau$ ทัр $\chi \tau \varepsilon \cup o v$.

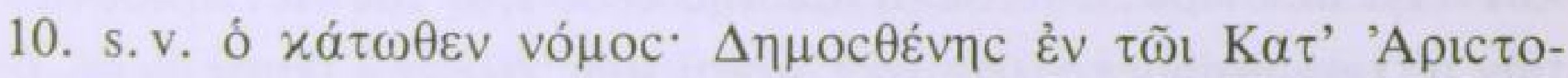

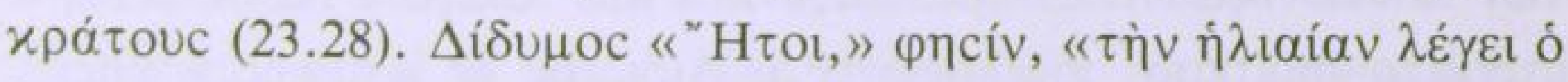

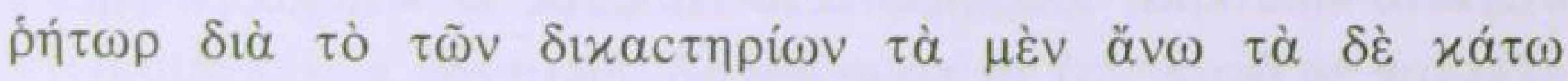

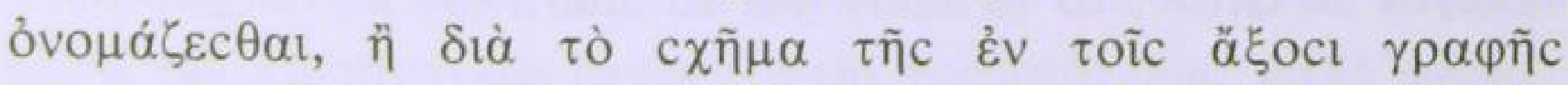

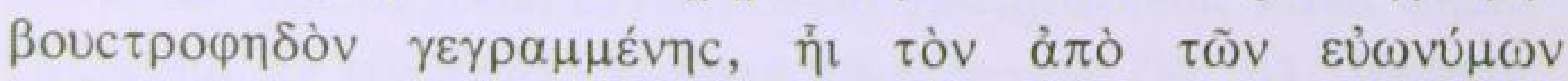

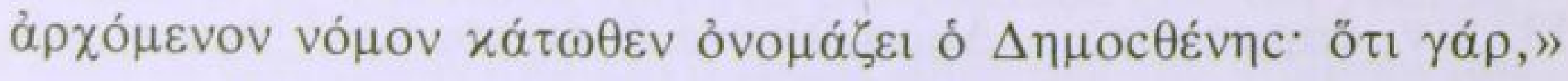

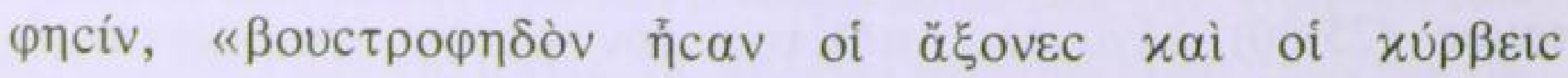

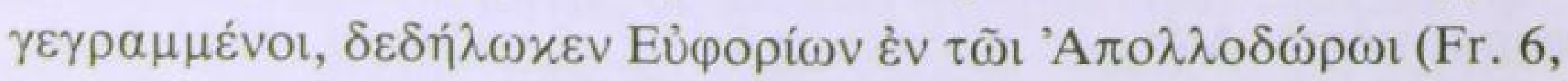
Powell, Coll. Alex.) 


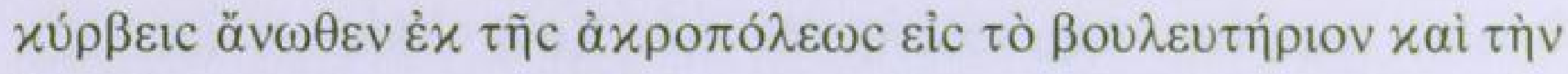

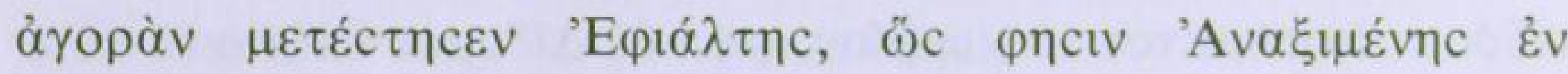
$\Phi เ \lambda เ \pi \pi \iota x \tilde{\omega} \iota)(\mathrm{FGrH} 72 \mathrm{~F} 13)$.

Cf. Lex. ad Dem. Aristocrateam B 7-24 (D-S ${ }^{2}$ p. 46) $\Delta i \delta$ í $\mu$ oc ó

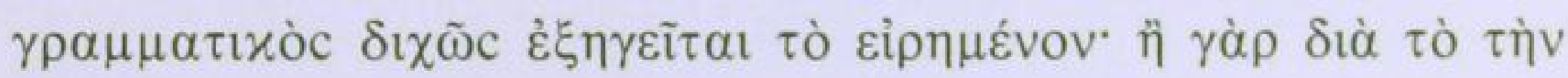

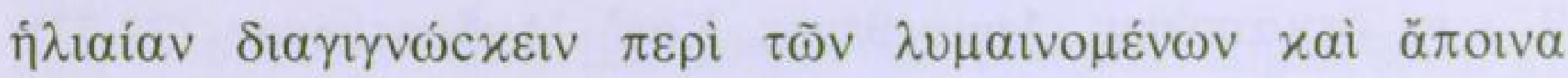

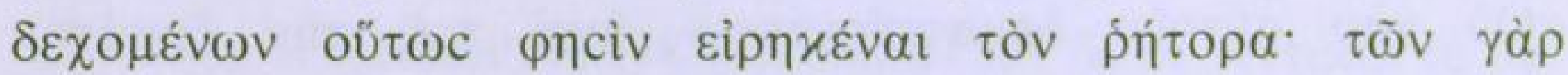

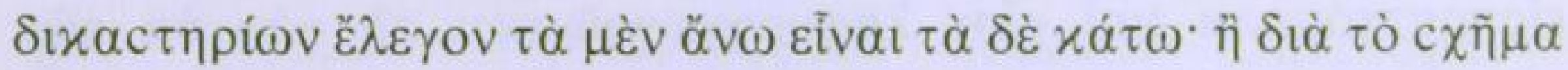
$\tau \tilde{\eta} c \dot{\varepsilon} v \tau o \tilde{c} c \ddot{\alpha} \xi o c l ~ \gamma \rho \alpha \varphi \tilde{\eta} c \quad x \tau \lambda$.

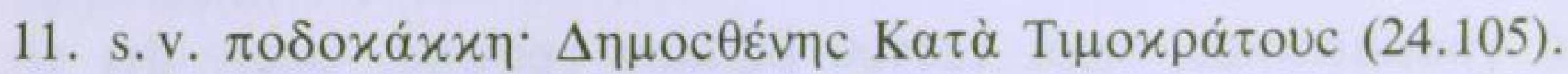

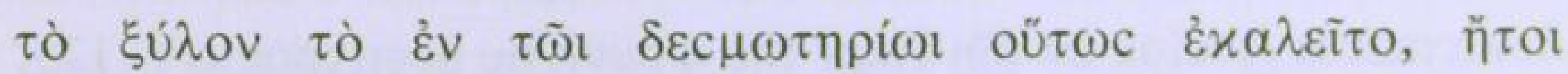

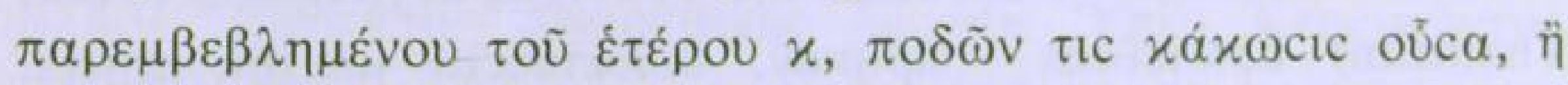

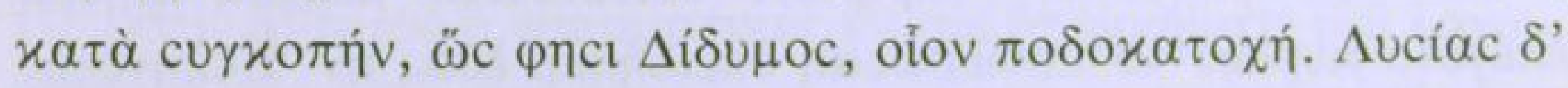

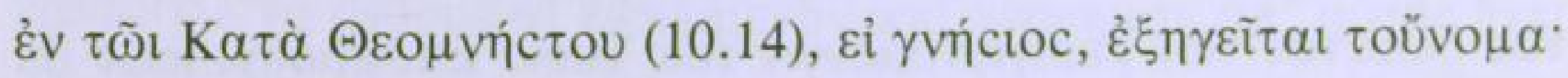

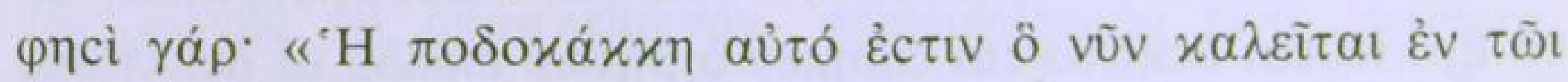
$\xi u ́ \lambda \omega \mathrm{\iota} \delta \varepsilon \delta \varepsilon \dot{c} \theta \alpha \imath . »$

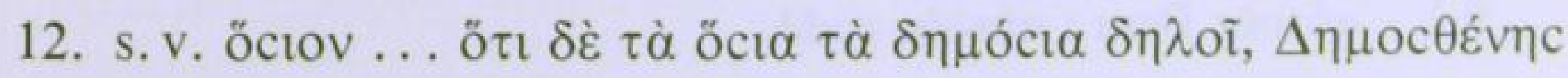

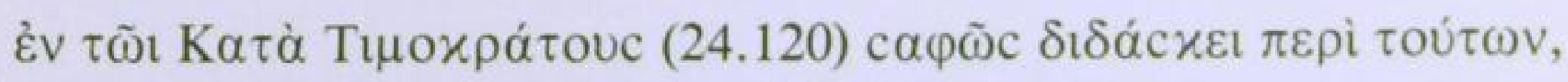

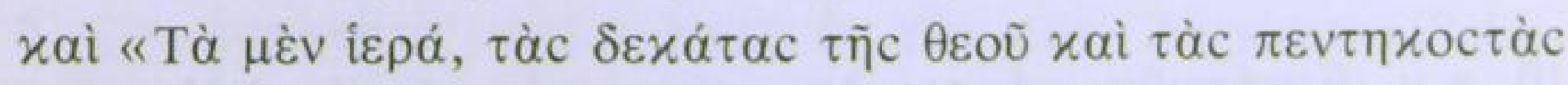

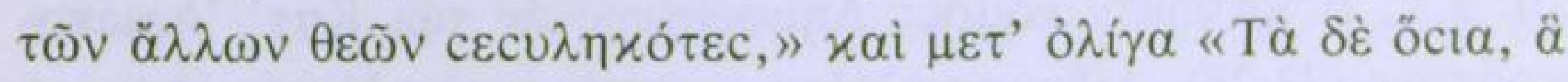

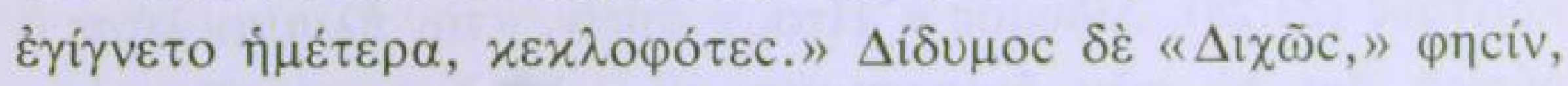

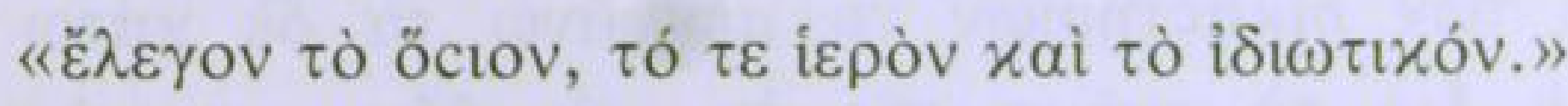

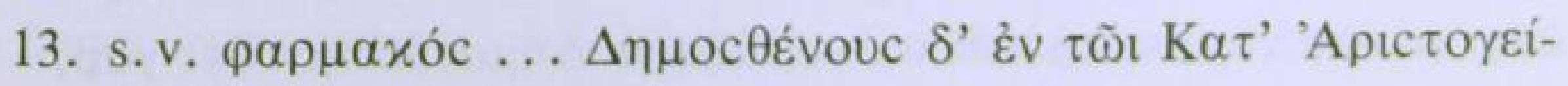

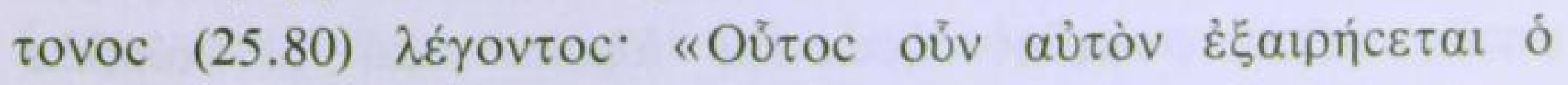

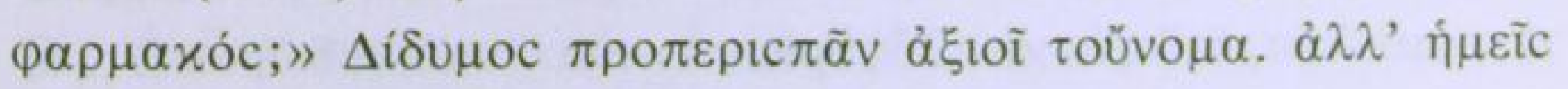

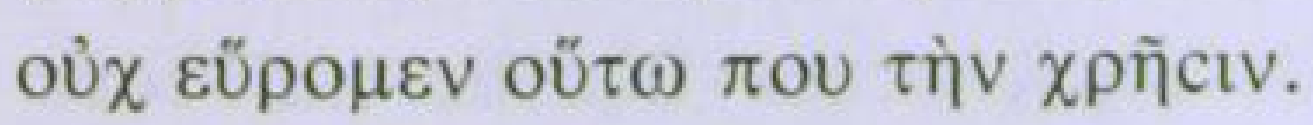




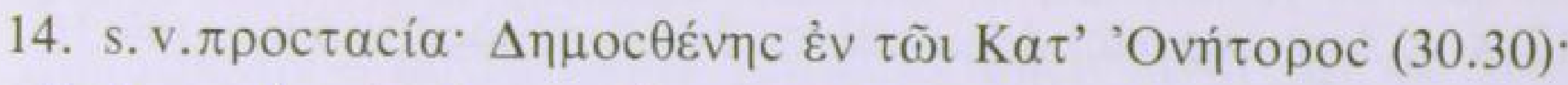

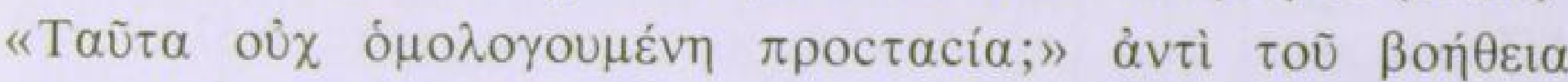

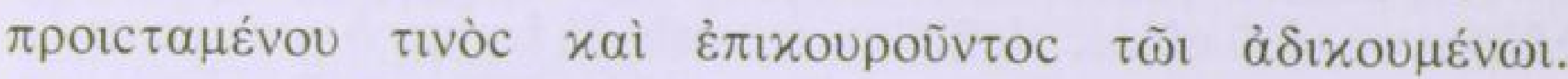

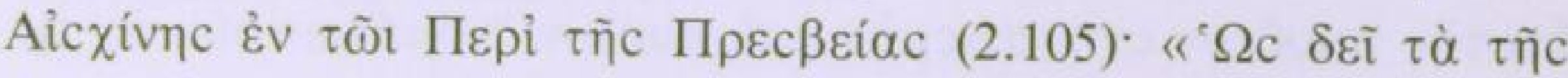

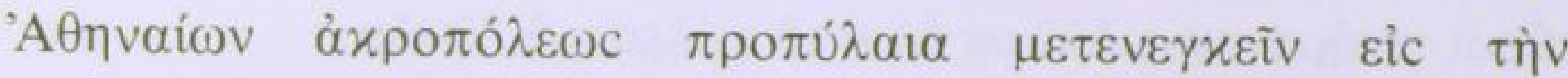

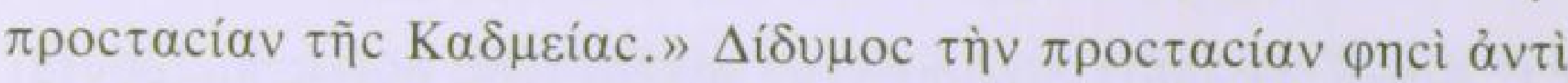

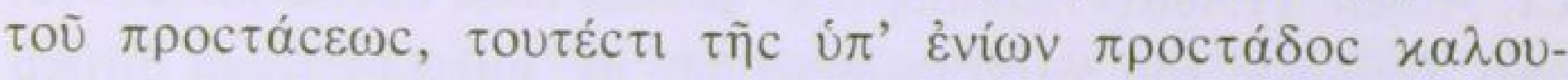
$\mu \varepsilon \dot{v \eta} \mathrm{c}$.

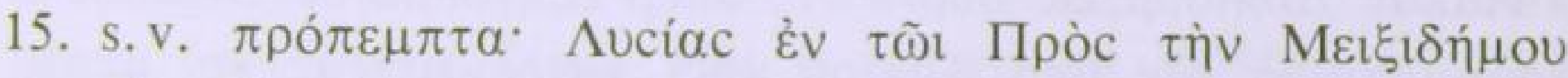

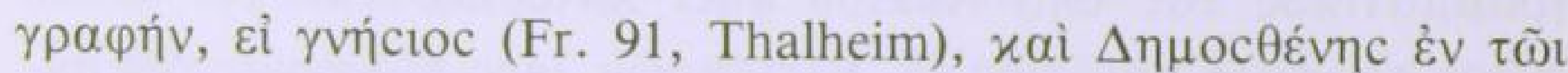

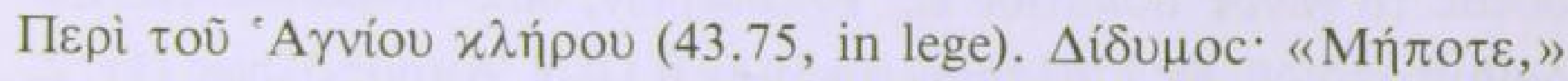

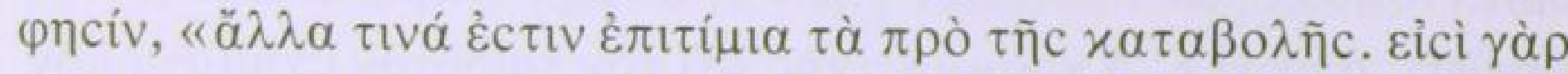

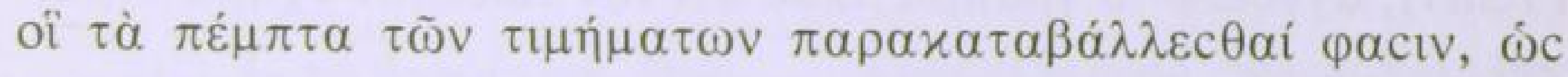

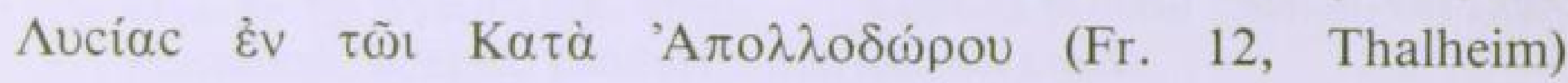

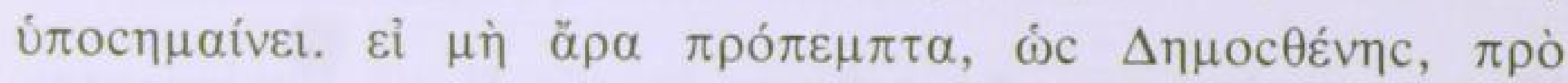

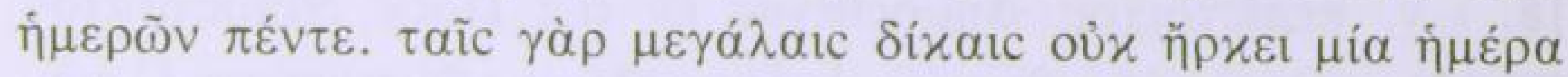

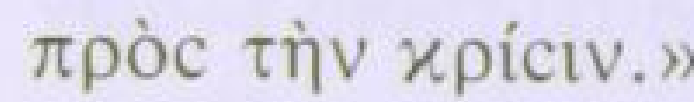

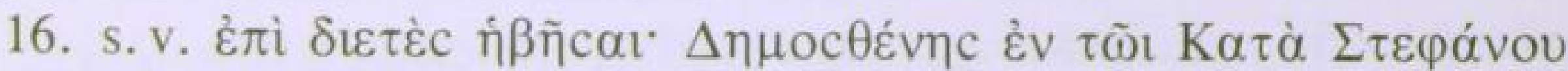

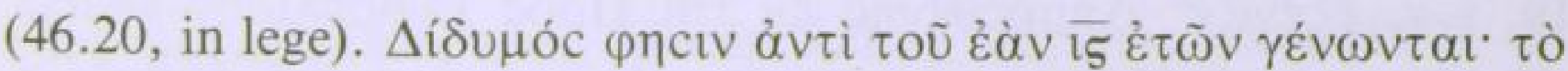

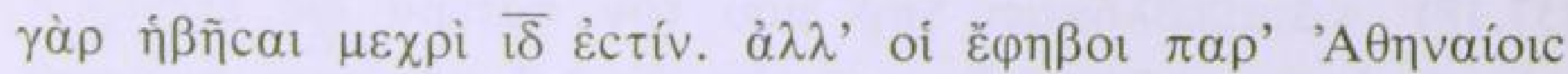

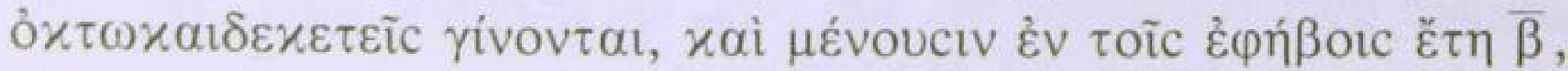

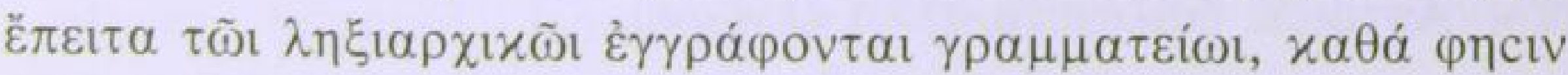

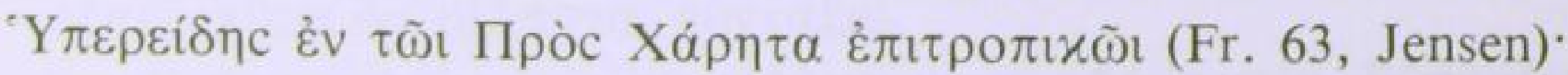

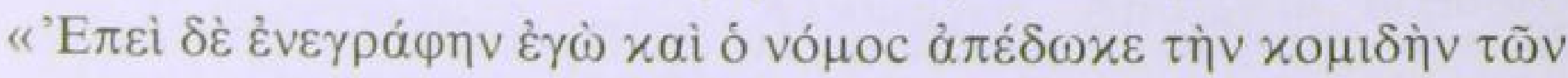

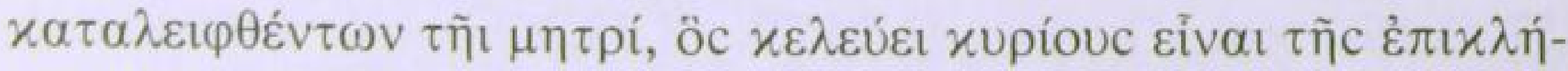

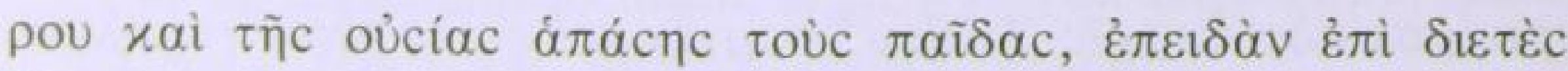

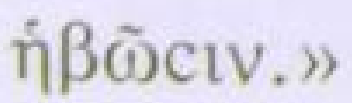




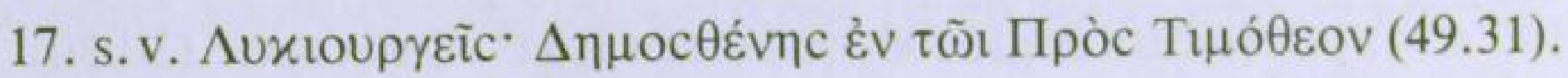

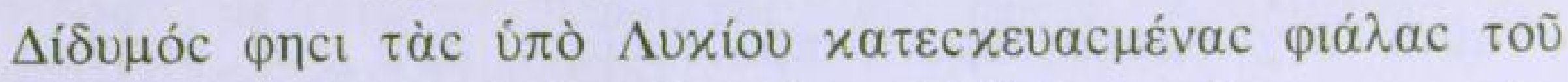

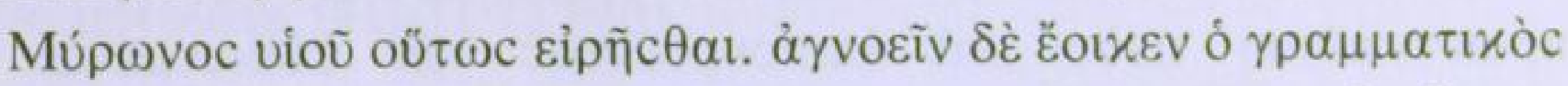

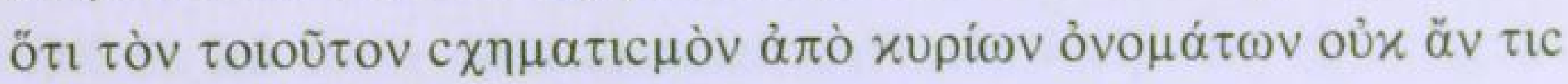

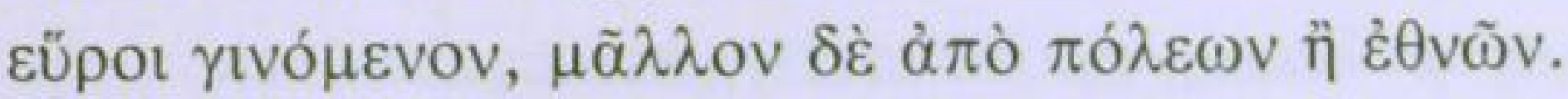

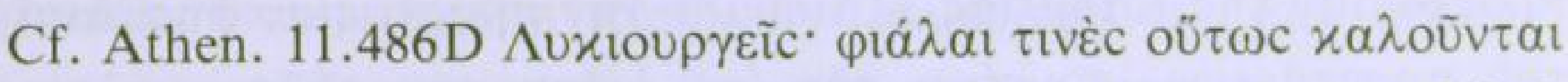

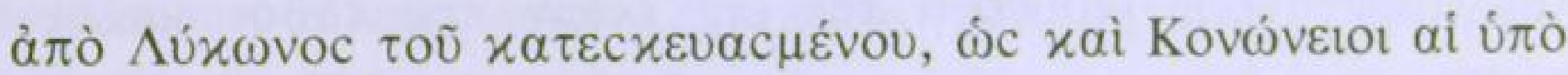

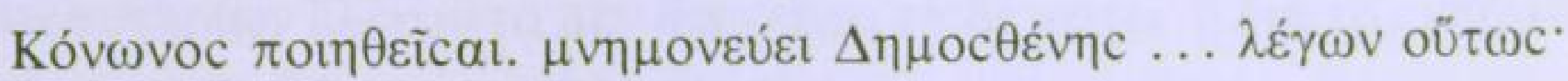

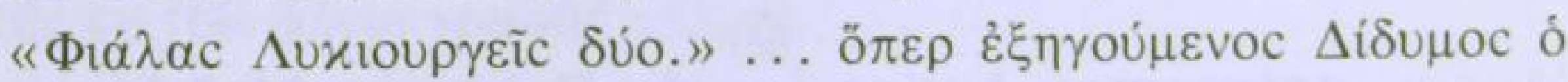

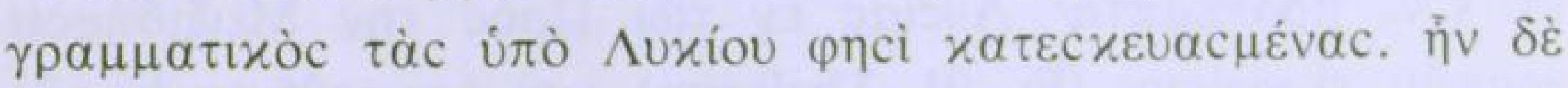

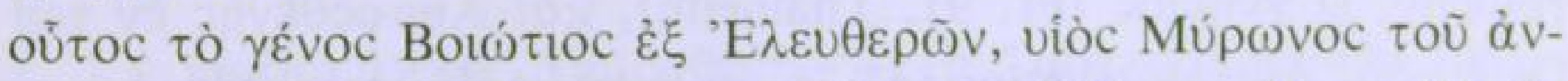

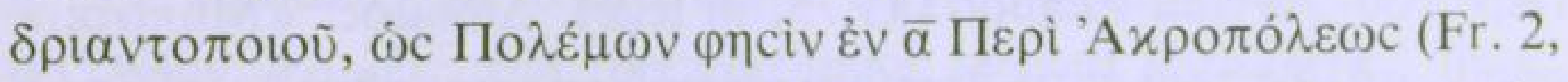

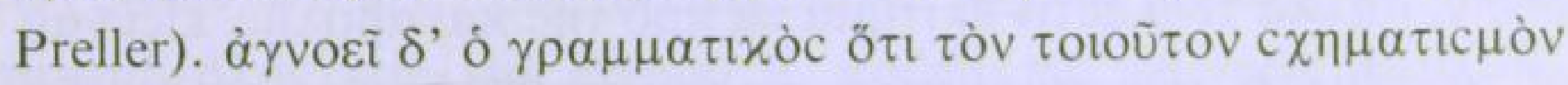
$x \tau \lambda$.

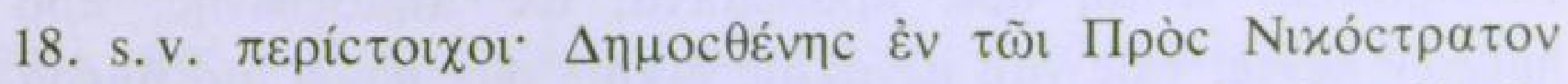

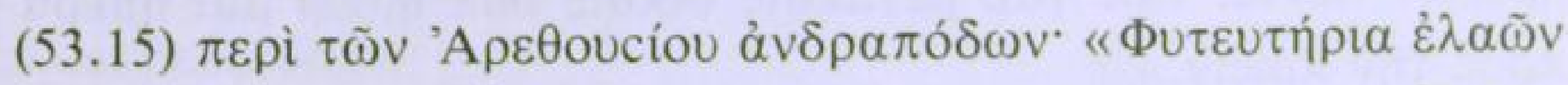

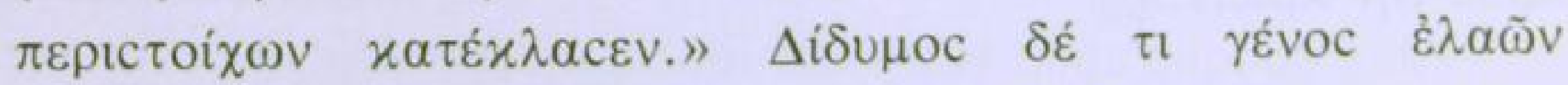

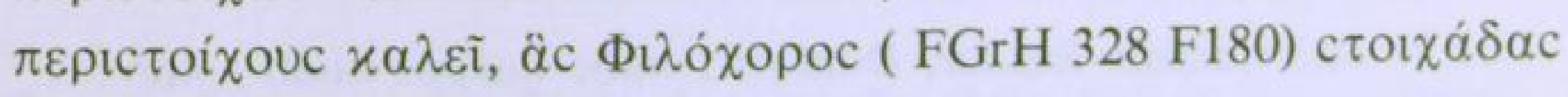

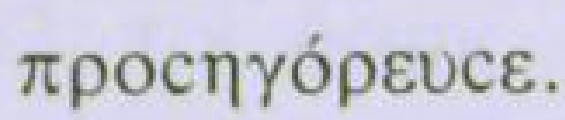

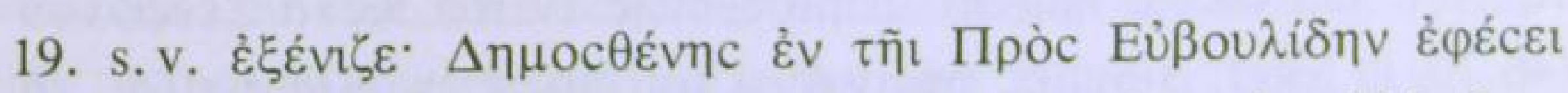

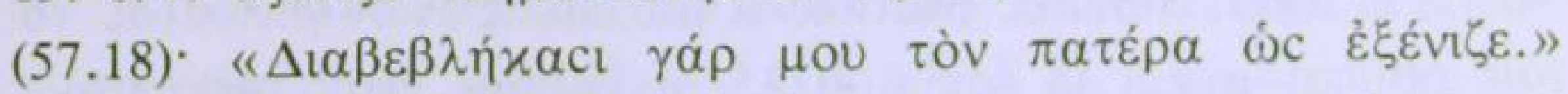

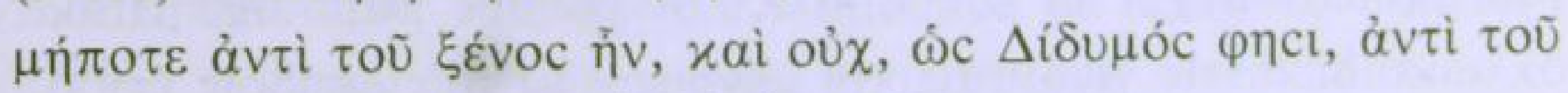

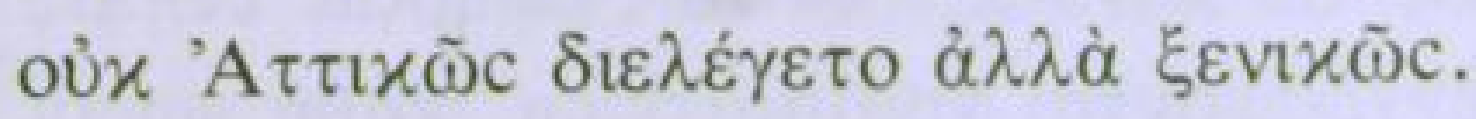

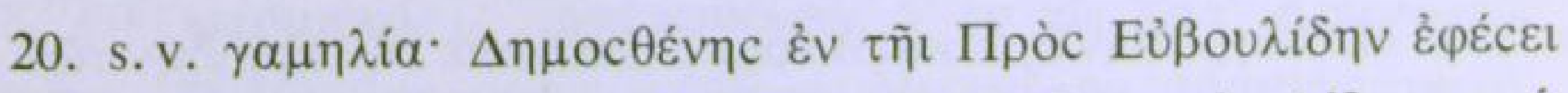

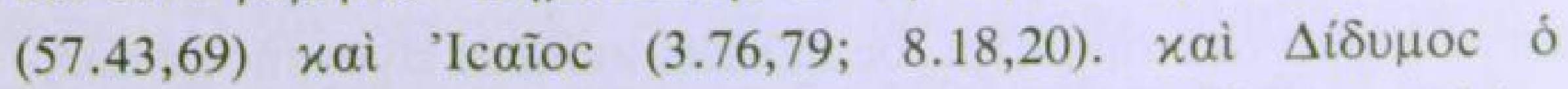

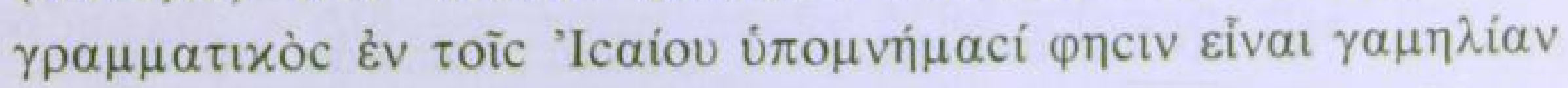

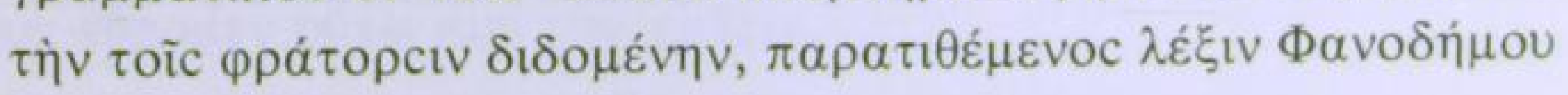




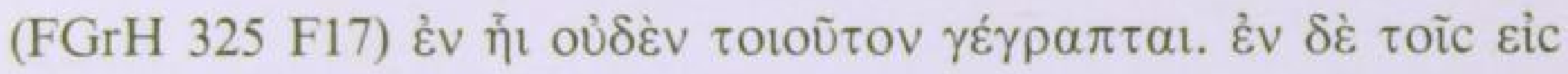

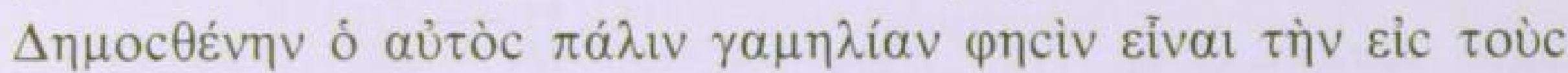

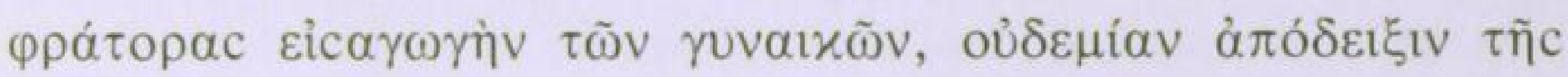
$\dot{\varepsilon} \xi \xi \gamma \eta \dot{c} \varepsilon \omega c \pi \alpha \rho \alpha \tau \imath \theta \varepsilon \dot{\varepsilon} \mu \varepsilon v o c$.

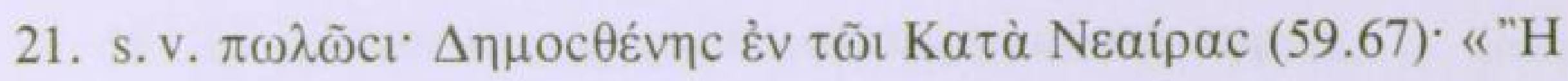

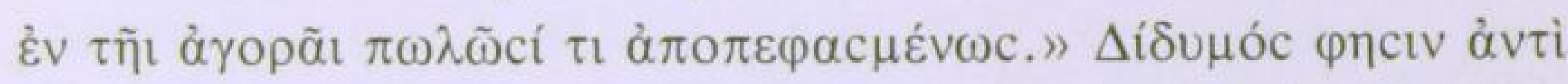

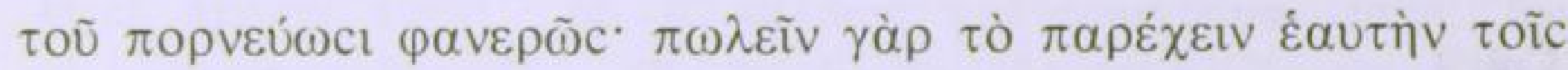

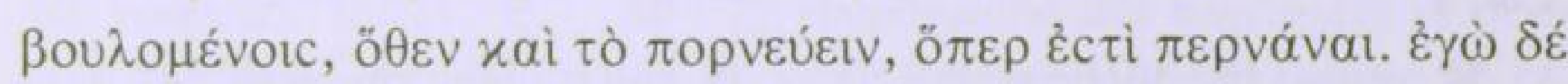

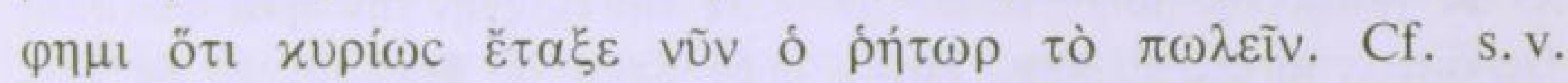

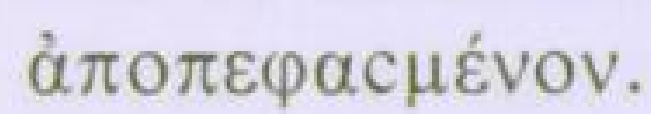


G Waentif 
